\title{
Orthogonal selection and fixing of coordination self- assembly pathways for robust metallo-organic ensemble construction
}

Michael J. Burke, ${ }^{\dagger}$ Gary S. Nichol, ${ }^{\dagger}$ and Paul J. Lusby. ${ }^{\dagger}, *$

${ }^{\dagger}$ EaStCHEM School of Chemistry, University of Edinburgh, The King’s Buildings, West Mains Road, Edinburgh EH9 3JJ, UK.

* Email: Paul.Lusby@ed.ac.uk

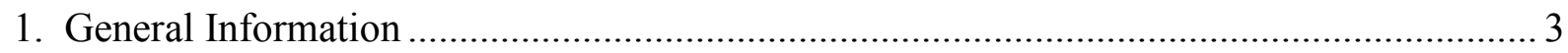

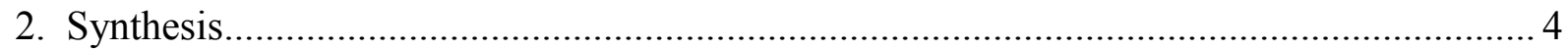

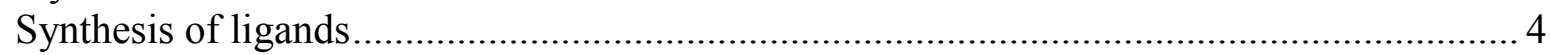

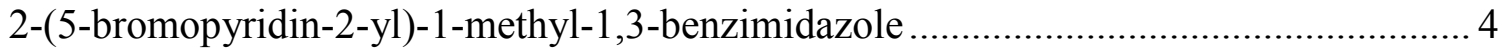

1,4-bis(6-(1-methyl-1,3-benzimidazolyl)pyridin-3-yl)benzene $\left(\mathbf{L}^{\mathbf{b}}\right)$.............................. 4

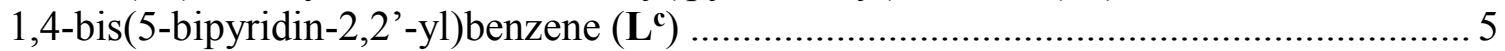

2,5-dimethylbenzene-1,4-diboronic acid bis(pinacol) ester .......................................... 5

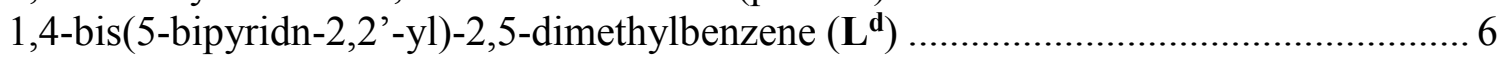

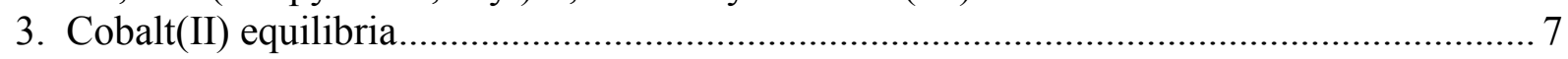

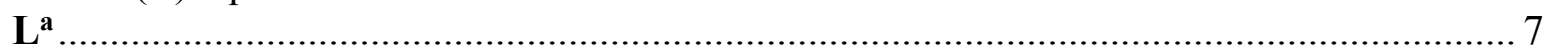

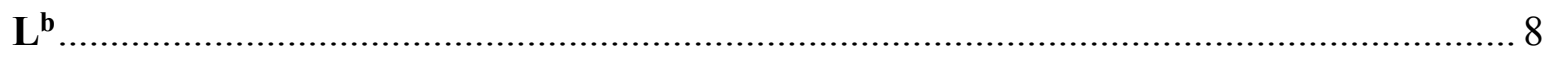

$\mathbf{L}^{\mathbf{c}}$

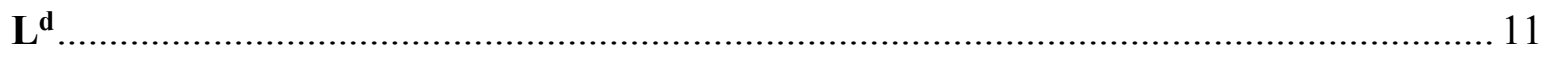

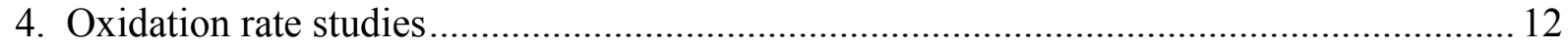

$\mathbf{L}^{\mathbf{a}}$

$\mathbf{L}^{\mathbf{b}}$

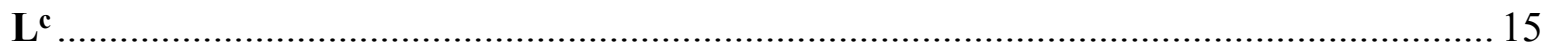

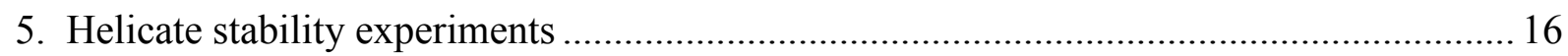

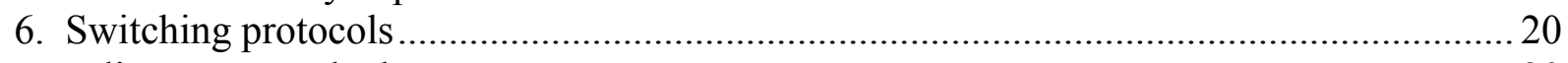

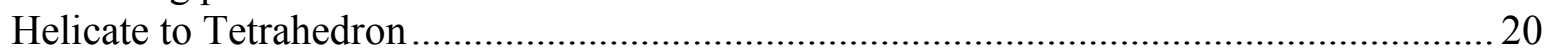

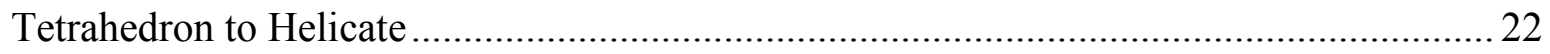

Photoredox induced rearrangement............................................................................. 24

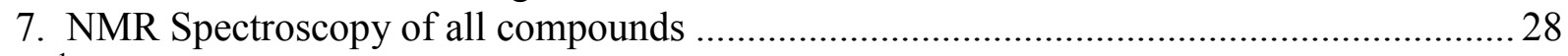

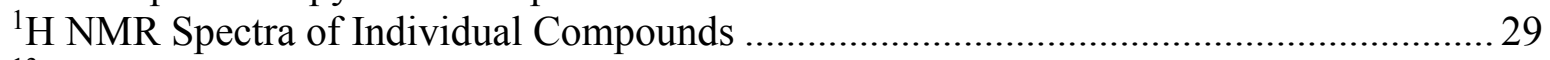

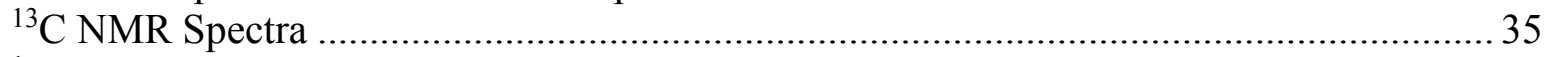

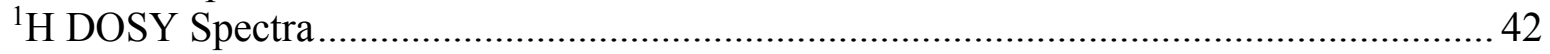

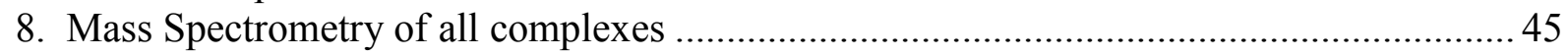

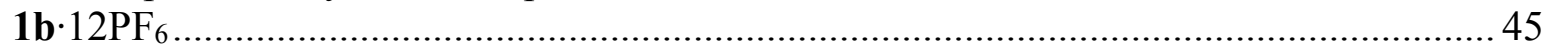

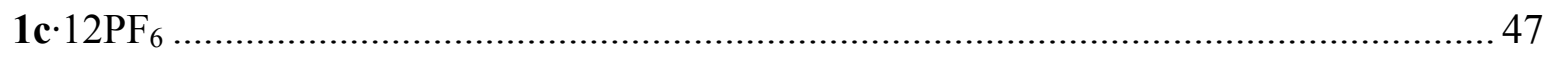

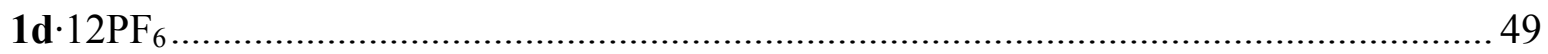

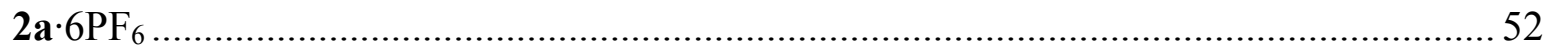

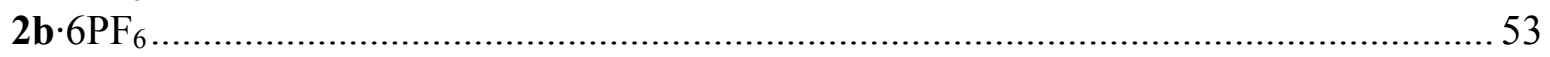

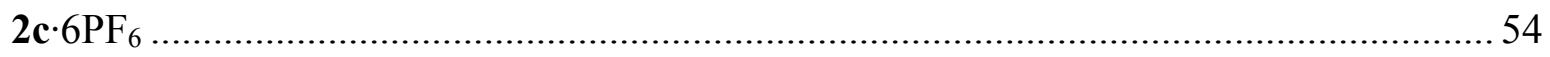


$\mathbf{2 d} \cdot 6 \mathrm{PF}_{6}$ 56

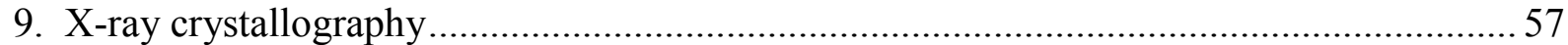

1b·12PF 6 Crystal Data (CCDC 1425917) and Experimental ................................................5 57

1d·12PF 6 (CCDC 1425919) Crystal Data and Experimental ................................................ 78

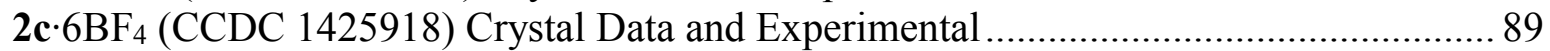

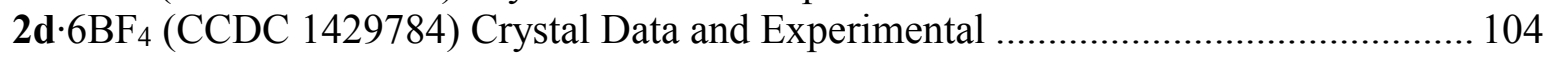

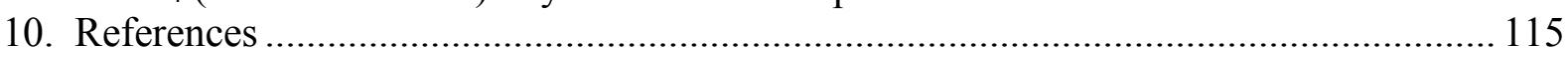




\section{General Information}

All reagents were purchased from Sigma-Aldrich, VWR or Alfa-Aesar and used without further purification. Where anhydrous solvent is stated, drying was carried out using a solvent purification system manufactured by Innovative Technology, Newburyport, MA, USA. Column chromatography was carried out using Kieselgel 60 (particle size 35-70) microns as the stationary phase and TLC was performed on precoated silica 60 gel plates $(0.20 \mathrm{~mm}$ thick, $60 \mathrm{~F}_{254}$. Merck, Germany) and observed under UV light. All reactions were carried out under $\mathrm{N}_{2}$ atmosphere, unless stated otherwise. Degassing of solvents was carried out using vacuum$\mathrm{N}_{2}$ backfill cycles on Schlenk apparatus. An ultrasonic bath was applied during the vacuum half of the cycle to enhance gas release from the liquid.

All ${ }^{1} \mathrm{H}$ and ${ }^{13} \mathrm{C}$ NMR spectra were recorded on either Bruker AV400, AV500, PRO500 or AV600 at a constant temperature of $300 \mathrm{~K}$ unless stated otherwise. All DOSY experiments were recorded on Bruker AV500 (Topspin 2.1) using bipolar gradient pulses for diffusion with two spoil gradients (ledbpg2s.compensated) pulse sequence. The sequence was carried out under automated conditions where the duration of the magnetic pulse gradient was $1.5 \mathrm{~ms}$ and the diffusion time was $100 \mathrm{~ms}$. Typically in each PFG NMR experiment, a series of 16 spectra on $32 \mathrm{~K}$ data points were collected and the eddy current delay was set to $5 \mathrm{~min}$ in all experiments. The pulse gradients were incremented from 2 to $95 \%$ of the maximum gradient strength in a linear ramp. The temperature was set and controlled at $300 \mathrm{~K}$ with an air flow of $400 \mathrm{~L} \mathrm{~h}^{-1}$ in order to avoid any temperature fluctuations due to sample heating during the magnetic field pulse gradients. The Stokes-Einstein equation was used to convert diffusion coefficient to hydrodynamic radius. The NMR data was processed using Bruker Topspin 2.1 and MestreLab Research MestReNova 6.0.3. Chemical shifts are reported in parts per million from low to high field and were referenced against values for the residual solvent peaks. Coupling constants $(J)$ are reported as observed in Hz. Standard abbreviations indicating multiplicity were used as follows: $\mathrm{m}=$ multiplet, $\mathrm{t}=$ triplet, $\mathrm{d}=$ doublet, $\mathrm{s}=\operatorname{singlet}, \mathrm{br}(\mathrm{s} / \mathrm{d})=$ broad (singlet/doublet etc.), appt = apparent triplet, etc.

Mass spectrometry of organic compounds was carried out on a high resolution Bruker ToF instrument; mass spectrometry (ESI-MS) of complexes was carried out using a Waters SYNAPT G2 instrument. 


\section{Synthesis}

\section{Synthesis of ligands}

Fragment 5-bromo-2,2'-bipyridine was synthesised according to a literature procedure. ${ }^{[1]}$ Ligand $\mathbf{L}^{\mathbf{a}}$ and tetrahedron $\mathbf{1} \mathbf{a} \cdot 12 \mathrm{PF}_{6}$ were synthesised as reported in a previous paper. ${ }^{[2]}$

\section{2-(5-bromopyridin-2-yl)-1-methyl-1,3-benzimidazole}<smiles>Fc1ccc(Br)cn1</smiles>

To a degassed suspension of 5-bromopicolinic acid (10.29 g, $50.9 \mathrm{mmol})$ in dry toluene (250 $\mathrm{mL}$ ) in an ice bath, borane-tetrahydrofuran adduct (1M in THF, $17.8 \mathrm{~mL}, 17.8 \mathrm{mmol}$ ) was added dropwise. After stirring for $1 \mathrm{~h}$, the solution was allowed to warm to room temperature and $N$-methyl-1,2-phenylenediamine $(1.90 \mathrm{~mL}, 16.7 \mathrm{mmol})$ was added. The solution was subsequently refluxed for 7 days. The solvent was removed under vacuum, the crude product purified by silica flash column $\left(\mathrm{CH}_{2} \mathrm{Cl}_{2}\right)$ and then recrystallised from dichloromethane-hexane to give the title compound as colourless needles. Yield $=2.935 \mathrm{~g}(61 \%)$. m.p. $141-143{ }^{\circ} \mathrm{C} .{ }^{1} \mathrm{H}$ NMR $\left(600 \mathrm{MHz}, \mathrm{CDCl}_{3}\right): \delta 8.75\left(\mathrm{~d}, J=2.3 \mathrm{~Hz}, 1 \mathrm{H}, \mathrm{H}_{H}\right), 8.33\left(\mathrm{~d}, J=8.5 \mathrm{~Hz}, 1 \mathrm{H}, \mathrm{H}_{F}\right), 7.96$ $\left(\mathrm{dd}, J=8.5 \mathrm{~Hz}, 2.4 \mathrm{~Hz}, 1 \mathrm{H}, \mathrm{H}_{G}\right), 7.82\left(\mathrm{~d}, J=7.9 \mathrm{~Hz}, 1 \mathrm{H}, \mathrm{H}_{A}\right), 7.43\left(\mathrm{~d}, J=7.9 \mathrm{~Hz}, 1 \mathrm{H}, \mathrm{H}_{D}\right)$, 7.35 (appt, $\left.1 \mathrm{H}, \mathrm{H}_{C}\right), 7.32$ (appt, $\left.J=7.5 \mathrm{~Hz}, 1 \mathrm{H}, \mathrm{H}_{B}\right), 4.25\left(\mathrm{~s}, 3 \mathrm{H}, \mathrm{H}_{E}\right) .{ }^{13} \mathrm{C}$ NMR $(151 \mathrm{MHz}$, $\left.\mathrm{CDCl}_{3}\right): \delta 149.8,149.4,149.2,142.6,139.6,137.5,126.0,123.8,123.0,121.4,120.2,110.1$, 33.0. HR-ESI MS: $m / z 288.01309$ (predicted $[\mathrm{M}+\mathrm{H}]^{+}=288.01363$ ), 309.99503 (predicted $\left.[\mathrm{M}+\mathrm{Na}]^{+}=309.99558\right)$.

\section{1,4-bis(6-(1-methyl-1,3-benzimidazolyl)pyridin-3-yl)benzene $\left(L^{b}\right)$}<smiles></smiles>

2-(5-bromopyridin-2-yl)-1-methyl-1,3-benzimidazole (1.591 g, $5.52 \mathrm{mmol}$ ), benzene-1,4diboronic acid $(0.447 \mathrm{~g}, 2.70 \mathrm{mmol})$, potassium carbonate $(1.864 \mathrm{~g}, 13.5 \mathrm{mmol})$ and palladium tetrakis(triphenylphosphine) $(0.156 \mathrm{~g}, 0.135 \mathrm{mmol})$ were combined in a mixture of degassed tetrahydrofuran $(27.0 \mathrm{~mL})$, water $(13.5 \mathrm{~mL})$ and ethanol $(22.5 \mathrm{~mL})$ and heated at 70 ${ }^{\circ} \mathrm{C}$ for $21 \mathrm{~h}$ under $\mathrm{N}_{2}$. This solution was then allowed to cool to room temperature and the precipitate isolated by filtration. The crude product was purified using a silica flash column $\left(1 \% \mathrm{CH}_{3} \mathrm{OH}\right.$ in $\left.\mathrm{CH}_{2} \mathrm{Cl}_{2}\right)$ to give the title compound as a colourless powder. Yield $=0.985 \mathrm{~g}$ (74\%). m.p. $172-174{ }^{\circ} \mathrm{C} .{ }^{1} \mathrm{H}$ NMR $\left(600 \mathrm{MHz}, \mathrm{CDCl}_{3}\right) \delta 9.02\left(\mathrm{dd}, J=2.4,0.7 \mathrm{~Hz}, 2 \mathrm{H}, \mathrm{H}_{H}\right)$, $8.54\left(\mathrm{dd}, J=8.2,0.7 \mathrm{~Hz}, 2 \mathrm{H}, \mathrm{H}_{F}\right), 8.13\left(\mathrm{dd}, J=8.2,2.4 \mathrm{~Hz}, 2 \mathrm{H}, \mathrm{H}_{G}\right), 7.87(\mathrm{dd}, J=7.4,1.3$ $\left.\mathrm{Hz}, 2 \mathrm{H}, \mathrm{H}_{A}\right), 7.84\left(\mathrm{~s}, 4 \mathrm{H}, \mathrm{H}_{I}\right), 7.48\left(\mathrm{dd}, J=7.7,1.3 \mathrm{~Hz}, 2 \mathrm{H}, \mathrm{H}_{D}\right), 7.41-7.35\left(\mathrm{~m}, 2 \mathrm{H}, \mathrm{H}_{C}\right)$, 7.37-7.31 (m, 2H, $\left.\mathrm{H}_{B}\right), 4.36\left(\mathrm{~s}, 6 \mathrm{H}, \mathrm{H}_{E}\right) .{ }^{13} \mathrm{C} \mathrm{NMR}\left(151 \mathrm{MHz}, \mathrm{CDCl}_{3}\right): \delta 150.2,149.9,147.0$, $142.9,137.6,137.5,135.7,135.1,128.1,124.9,123.6,122.9,120.24,110.1,33.0$. HR-ESI 
MS: $m / z 493.21120$ (predicted $\left.[\mathrm{M}+\mathrm{H}]^{+}=493.21352\right), 515.19230$ (predicted $[\mathrm{M}+\mathrm{Na}]^{+}=$ 515.19547).

\section{1,4-bis(5-bipyridin-2,2'-yl)benzene $\left(L^{c}\right)$}<smiles>Fc1cccc(-c2ccc(-c3ccc(-c4ccccn4)nc3)cc2)n1</smiles>

5-bromo-2,2'-bipyridine $(0.579 \mathrm{~g}, 2.46 \mathrm{mmol})$, benzene-1,4-diboronic acid $(0.200 \mathrm{~g}, 1.21$ $\mathrm{mmol})$, potassium carbonate $(0.851 \mathrm{~g}, 6.16 \mathrm{mmol})$ and palladium tetrakis(triphenylphosphine) $(0.142 \mathrm{~g}, 0.123 \mathrm{mmol})$ were combined in a mixture of degassed tetrahydrofuran $(12.0 \mathrm{~mL})$, water $(6.0 \mathrm{~mL})$ and ethanol $(10.0 \mathrm{~mL})$ and heated at $80{ }^{\circ} \mathrm{C}$ for $20 \mathrm{~h}$ under $\mathrm{N}_{2}$. This solution was then allowed to cool to room temperature, diluted with water $(5.0 \mathrm{~mL})$ and the precipitate isolated by filtration. The crude product was purified using a silica flash column $\left(1 \% \mathrm{Et}_{3} \mathrm{~N}\right.$ in $\mathrm{CH}_{2} \mathrm{Cl}_{2}$ ) to give the title compound as a colourless powder. Yield $=0.395 \mathrm{~g}(85 \%)$. m.p. 292$295{ }^{\circ} \mathrm{C} .{ }^{1} \mathrm{H}$ NMR $\left(500 \mathrm{MHz}, \mathrm{CDCl}_{3}\right): \delta 9.00\left(\mathrm{dd}, J=2.4,0.7 \mathrm{~Hz}, 2 \mathrm{H}, \mathrm{H}_{G}\right), 8.72(\mathrm{ddd}, J=4.8$, $\left.1.7,0.9 \mathrm{~Hz}, 2 \mathrm{H}, \mathrm{H}_{A}\right), 8.52\left(\mathrm{dd}, J=8.2,0.7 \mathrm{~Hz}, 2 \mathrm{H}, \mathrm{H}_{E}\right), 8.50-8.44\left(\mathrm{~m}, 2 \mathrm{H}, \mathrm{H}_{D}\right), 8.09$ (dd, $J=$ 8.2, $\left.2.4 \mathrm{~Hz}, 2 \mathrm{H}, \mathrm{H}_{F}\right), 7.88-7.83\left(\mathrm{~m}, 2 \mathrm{H}, \mathrm{H}_{C}\right), 7.81\left(\mathrm{~s}, 4 \mathrm{H}, \mathrm{H}_{H}\right), 7.36$ (ddd, J= 7.5, 4.8, $1.2 \mathrm{~Hz}$, $\left.2 \mathrm{H}, \mathrm{H}_{B}\right) .{ }^{13} \mathrm{C} \mathrm{NMR}\left(151 \mathrm{MHz}, \mathrm{CDCl}_{3}\right): \delta 155.4,154.7,149.0,147.5,137.5,137.4,135.8$, $135.4,127.9,124.0,121.4,121.4$. HR-ESI MS: $m / z 387.16070$ (predicted $[\mathrm{M}+\mathrm{H}]^{+}=$ $387.16042)$.

\section{2,5-dimethylbenzene-1,4-diboronic acid bis(pinacol) ester}

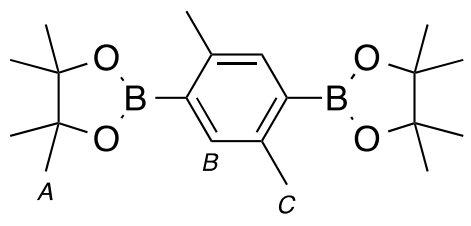

1,4-dibromo-2,5-dimethyl benzene (0.992 g, $3.76 \mathrm{mmol})$, bis(pinacolato)diboron (2.002 $\mathrm{g}$, $7.89 \mathrm{mmol})$, potassium acetate $(1.846 \mathrm{~g}, 18.8 \mathrm{mmol})$ and palladium bis-chloride diphenylphosphinoferrocene $(0.153 \mathrm{~g}, 0.188 \mathrm{mmol})$ was heated in degassed 1,4-dioxane (30 $\mathrm{mL}$ ) at $90{ }^{\circ} \mathrm{C}$ for $48 \mathrm{~h}$ under $\mathrm{N}_{2}$. This solution was then allowed to cool to room temperature and the solvent removed under reduced pressure. The crude product was purified using flash silica column chromatography $\left(2: 1\right.$ hexane $\left./ \mathrm{CH}_{2} \mathrm{Cl}_{2}\right)$ to give the title compound as a colourless crystalline solid. Yield $=0.938 \mathrm{~g}(70 \%)$. m.p. $271-274{ }^{\circ} \mathrm{C} .{ }^{1} \mathrm{H}$ NMR $\left(500 \mathrm{MHz}, \mathrm{CDCl}_{3}\right): \delta$ $7.54\left(\mathrm{~s}, 2 \mathrm{H}, \mathrm{H}_{B}\right), 2.48\left(\mathrm{~s}, 6 \mathrm{H}, \mathrm{H}_{C}\right), 1.34\left(\mathrm{~s}, 24 \mathrm{H}, \mathrm{H}_{A}\right) .{ }^{13} \mathrm{C} \mathrm{NMR}\left(126 \mathrm{MHz}, \mathrm{CDCl}_{3}\right): \delta 140.7$, 137.1, 130.9 $, 83.6,25.0,21.6 .{ }^{11} \mathrm{~B}$ NMR $\left(160 \mathrm{MHz}, \mathrm{CDCl}_{3}\right): \delta$ 31.2. HR-ESI MS: $\mathrm{m} / z$ $359.25510\left(\right.$ predicted $\left.[\mathrm{M}+\mathrm{H}]^{+}=359.25595\right), 381.23680\left(\right.$ predicted $\left.[\mathrm{M}+\mathrm{Na}]^{+}=381.23789\right)$. 


\section{1,4-bis(5-bipyridn-2,2'-yl)-2,5-dimethylbenzene $\left(L^{d}\right)$}<smiles>Cc1cc(-c2ccc(-c3ccccn3)nc2)c(I)cc1-c1ccc(-c2ccccn2)nc1</smiles>

5-bromo-2,2'-bipyridine $(0.427 \mathrm{~g}, 1.82 \mathrm{mmol}), 2,5$-dimethyl-1,4-benzene diboronic acid bis(pinacol) ester $(0.325 \mathrm{~g}, 0.908 \mathrm{mmol})$, potassium carbonate $(0.628 \mathrm{~g}, 4.54 \mathrm{mmol})$ and palladium tetrakis(triphenylphosphine) $(0.0525 \mathrm{~g}, 45.4 \mu \mathrm{mol})$ were combined in a mixture of degassed tetrahydrofuran $(9.0 \mathrm{~mL})$, water $(4.5 \mathrm{~mL})$ and ethanol $(7.0 \mathrm{~mL})$ and heated at $80{ }^{\circ} \mathrm{C}$ for $16 \mathrm{~h}$ under $\mathrm{N}_{2}$. This solution was then allowed to cool to room temperature, diluted with water $(4.0 \mathrm{~mL})$ and the precipitate isolated by filtration. The crude product was purified using a silica flash column ( $1 \%$ triethylamine in dichloromethane) to give the title compound as a colourless crystalline solid. Yield $=0.371 \mathrm{~g}(98 \%)$. m.p. $198-200{ }^{\circ} \mathrm{C} .{ }^{1} \mathrm{H}$ NMR $(500 \mathrm{MHz}$, $\left.\mathrm{CDCl}_{3}\right): \delta$ 8.74-8.71 (m, $\left.4 \mathrm{H}, \mathrm{H}_{G} \& \mathrm{H}_{A}\right), 8.49\left(\mathrm{dd}, J=8.1,0.7 \mathrm{~Hz}, 2 \mathrm{H}, \mathrm{H}_{E}\right), 8.48-8.43(\mathrm{~m}, 2 \mathrm{H}$, $\left.\mathrm{H}_{D}\right), 7.88-7.84\left(\mathrm{~m}, 4 \mathrm{H}, \mathrm{H}_{C} \& \mathrm{H}_{F}\right), 7.34$ (ddd, $\left.J=7.5,4.8,1.2 \mathrm{~Hz}, 2 \mathrm{H}, \mathrm{H}_{B}\right), 7.26\left(\mathrm{~s}, 2 \mathrm{H}, \mathrm{H}_{H}\right)$, $2.36\left(\mathrm{~s}, 6 \mathrm{H}, \mathrm{H}_{I}\right) .{ }^{13} \mathrm{C} \mathrm{NMR}\left(126 \mathrm{MHz}, \mathrm{CDCl}_{3}\right): \delta 156.2,154.94,149.5,149.5,138.0,137.6$, 137.2, 137.1, 133.6, 132.3, 123.9, 121.2, 120.7, 20.1. HR-ESI MS: $m / z 415.19130$ (predicted $\left.[\mathrm{M}+\mathrm{H}]^{+}=415.19172\right)$.

$\dagger$ The quaternary carbon attached to boron was identified by ${ }^{1} \mathrm{H}-{ }^{13} \mathrm{C}$ HMBC NMR through long range coupling to protons, as the quadrupolar relaxation of the boron nucleus causes significant $\mathrm{T}_{2}$ broadening in adjacent atoms 


\section{Cobalt(II) equilibria}

The cobalt(II) equilibria were studied by ${ }^{1} \mathrm{H}$ NMR spectroscopy. Samples were obtained by combining $\mathrm{Co}\left(\mathrm{ClO}_{4}\right)_{2} \cdot 6 \mathrm{H}_{2} \mathrm{O}$ with the appropriate ligand $\left(\mathbf{L}^{\mathrm{a}-\mathbf{d}}\right)$ in a $2: 3$ ratio in $\mathrm{CD}_{3} \mathrm{CN}$, followed by heating at $50{ }^{\circ} \mathrm{C}$ for $15-30$ minutes to ensure complete dissolution. The samples were allowed to cool to RT before the first NMR spectrum was recorded. The NMR spectra of this and subsequent dilutions were checked over a number of days until no further change was observed.

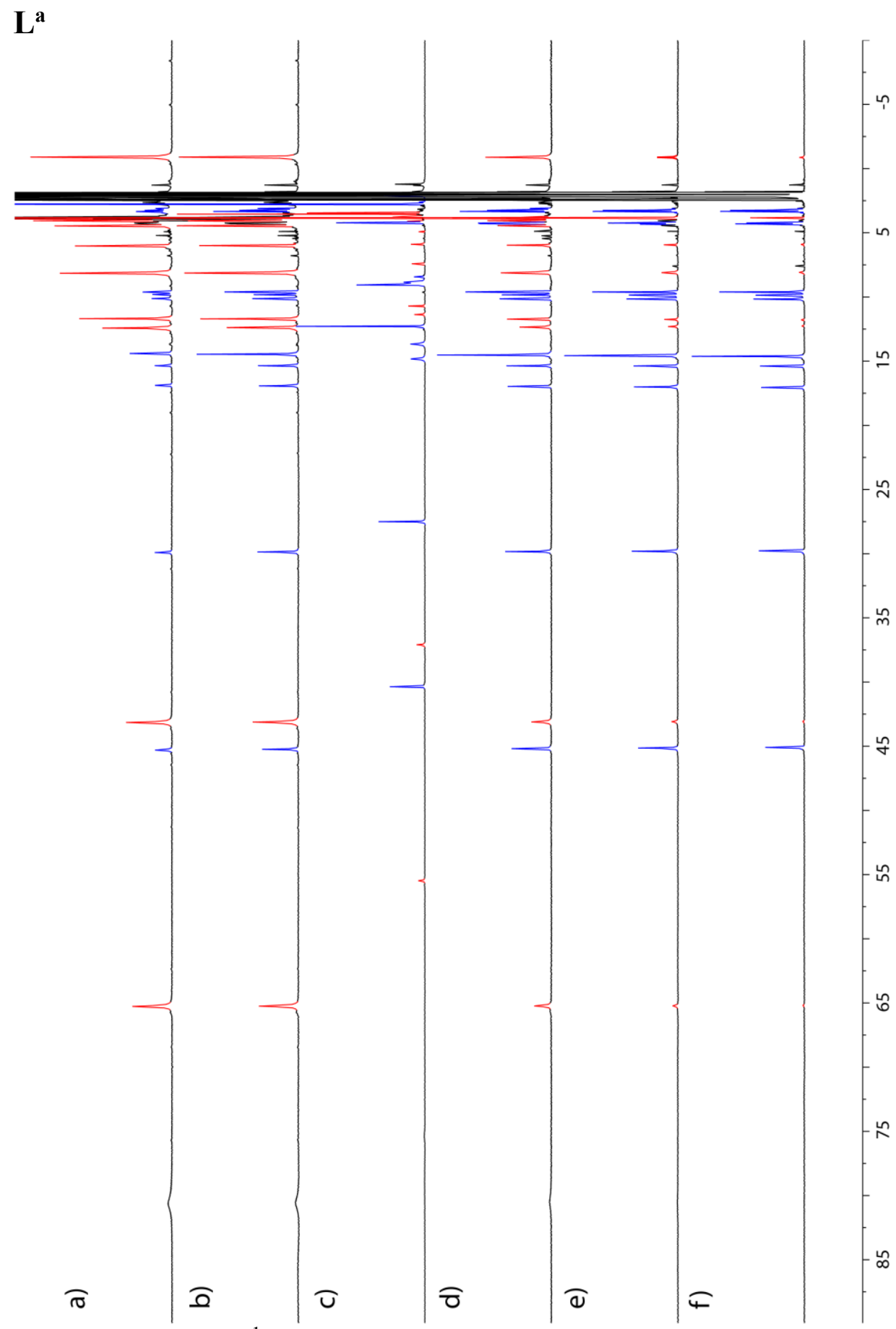

Figure S1: Partial ${ }^{1} \mathrm{H}$ NMR Spectra (500 MHZ, $\mathrm{CD}_{3} \mathrm{CN}, 300 \mathrm{~K}$ unless stated) showing the equilibrium between $\mathrm{Co}_{2}{ }_{2} \mathbf{L}_{3}{ }_{3}^{4+}\left(\mathbf{4 a} \cdot 4 \mathrm{ClO}_{4}\right.$, blue) and $\mathrm{Co}_{4}{ }_{4} \mathbf{L}_{6}{ }_{6}{ }^{8+}\left(3 \mathbf{a} \cdot 8 \mathrm{ClO}_{4}\right.$, red $)$ as a function 
of $[\mathrm{Co}]_{\text {total }}$ and temperature. a) $\left[\mathrm{Co}^{\mathrm{II}}\right]_{\text {total }}=11.7 \mathrm{mM}$, b) $\left[\mathrm{Co}^{\mathrm{II}}\right]_{\text {total }}=5.84 \mathrm{mM}$, c) $\left[\mathrm{Co}^{\mathrm{II}}\right]_{\text {total }}=$ $5.84 \mathrm{mM}$ at $\left.\left.343 \mathrm{~K}, \mathrm{~d})\left[\mathrm{Co}^{\mathrm{II}}\right]_{\text {total }}=2.92 \mathrm{mM}, \mathrm{e}\right)\left[\mathrm{Co}^{\mathrm{II}}\right]_{\text {total }}=1.46 \mathrm{mM}, \mathrm{f}\right)\left[\mathrm{Co}^{\mathrm{II}}\right]_{\text {total }}=0.73 \mathrm{mM}$.

$\mathbf{L}^{\mathbf{b}}$

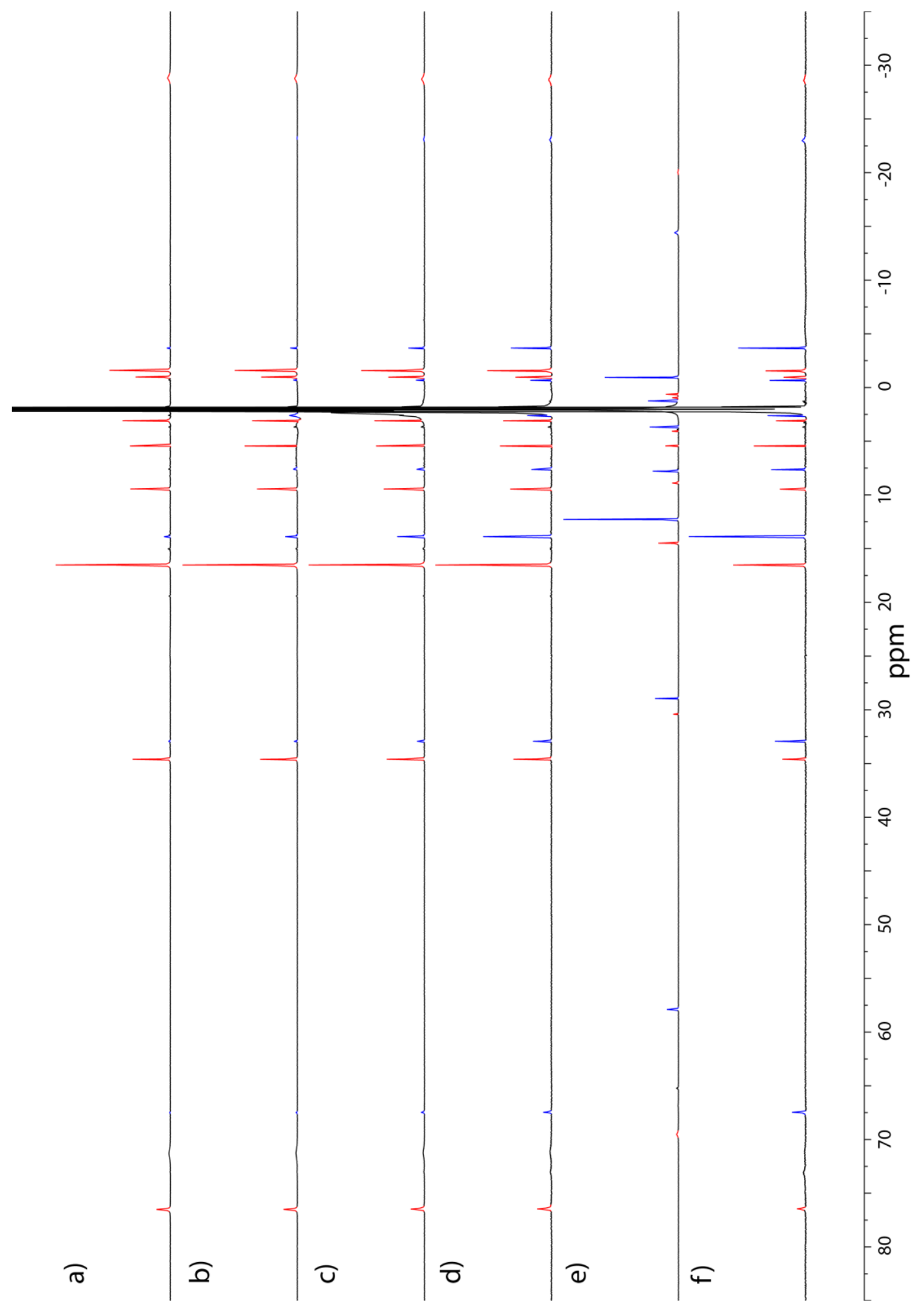

Figure S2: Partial ${ }^{1} \mathrm{H}$ NMR Spectra (500 MHZ, $\mathrm{CD}_{3} \mathrm{CN}, 300 \mathrm{~K}$ unless stated) showing the equilibrium between $\mathrm{Co}_{2}{ }_{2} \mathbf{L}_{3}^{\mathbf{b}_{3}{ }^{4+}}\left(\mathbf{4 b} \cdot 4 \mathrm{ClO}_{4}\right.$, blue $)$ and $\mathrm{Co}_{4}{ }_{4} \mathbf{L}_{6}^{\mathbf{b}_{6}{ }^{8+}}\left(\mathbf{3} \mathbf{b} \cdot 8 \mathrm{ClO}_{4}\right.$, red) as a function 
of $[\mathrm{Co}]_{\text {total }}$ and temperature. a) $\left[\mathrm{Co}^{\mathrm{II}}\right]_{\text {total }}=11.6 \mathrm{mM}$, b) $\left[\mathrm{Co}^{\mathrm{II}}\right]_{\text {total }}=5.82 \mathrm{mM}$, c) $\left[\mathrm{Co}^{\mathrm{II}}\right]_{\text {total }}=$ $2.91 \mathrm{mM}, \mathrm{d})\left[\mathrm{Co}^{\mathrm{II}}\right]_{\text {total }}=1.45 \mathrm{mM}$, e) $\left[\mathrm{Co}^{\mathrm{II}}\right]_{\text {total }}=1.45 \mathrm{mM}$ at $\left.343 \mathrm{~K}, \mathrm{f}\right)\left[\mathrm{Co}^{\mathrm{II}}\right]_{\text {total }}=0.73 \mathrm{mM}$.

\section{$\mathbf{L}^{\mathbf{c}}$}

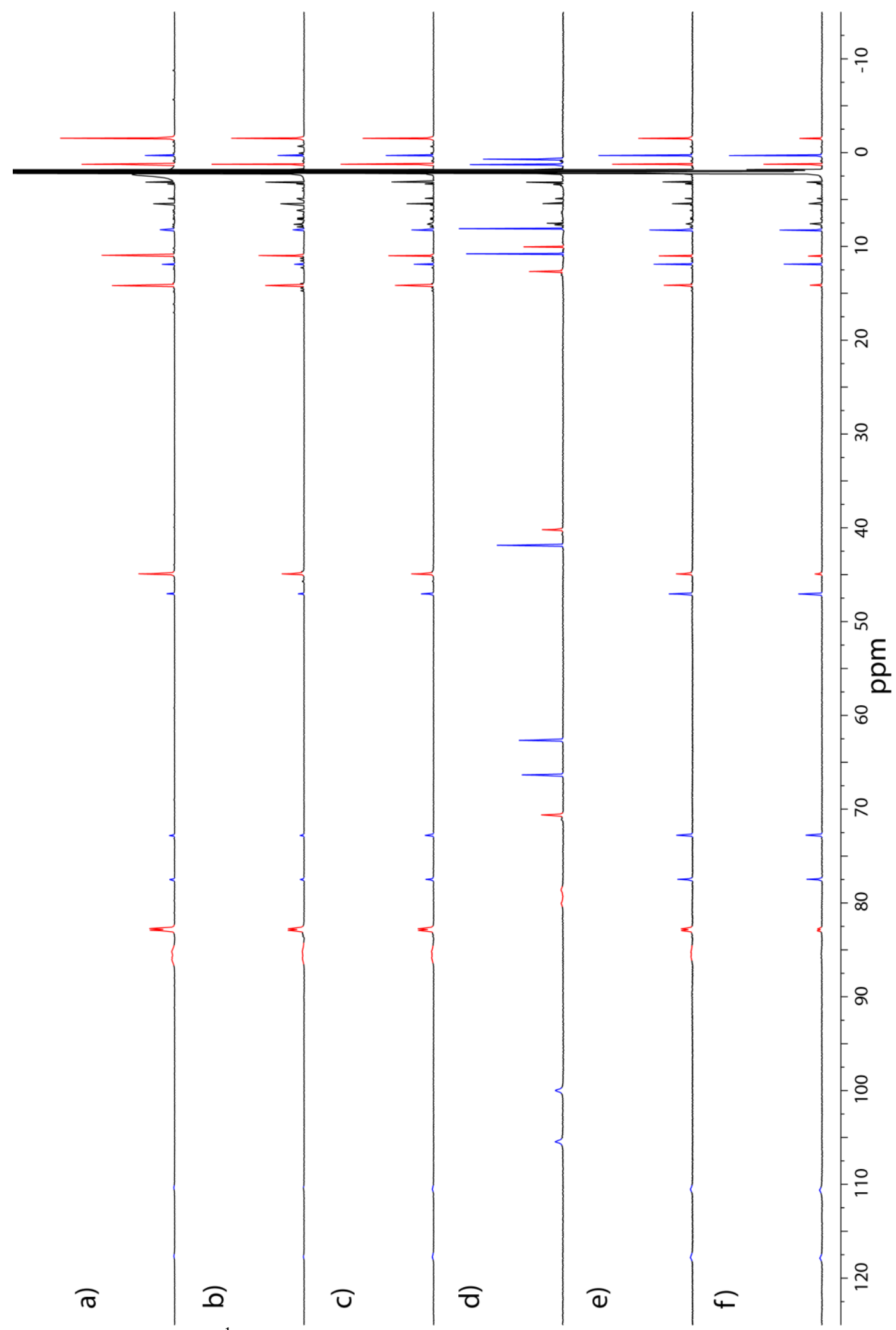

Figure S3: Partial ${ }^{1} \mathrm{H}$ NMR Spectra (500 MHZ, $\mathrm{CD}_{3} \mathrm{CN}, 300 \mathrm{~K}$ unless stated) showing the equilibrium between $\mathrm{Co}_{2}{ }_{2} \mathbf{L}_{3}{ }^{\mathbf{c}^{4+}}\left(\mathbf{4} \mathbf{c} \cdot 4 \mathrm{ClO}_{4}\right.$, blue) and $\mathrm{Co}_{4}{ }_{4} \mathbf{L}_{6}{ }^{{ }^{8+}}\left(3 \mathbf{c} \cdot 8 \mathrm{ClO}_{4}\right.$, red) as a function 
of $[\mathrm{Co}]_{\text {total }}$ and temperature. a) $\left[\mathrm{Co}^{\mathrm{II}}\right]_{\text {total }}=11.6 \mathrm{mM}$, b) $\left[\mathrm{Co}^{\mathrm{II}}\right]_{\text {total }}=5.82 \mathrm{mM}$, c) $\left[\mathrm{Co}^{\mathrm{II}}\right]_{\text {total }}=$ $2.91 \mathrm{mM}, \mathrm{d})\left[\mathrm{Co}^{\mathrm{II}}\right]_{\text {total }}=2.91 \mathrm{mM}$ at $343 \mathrm{~K}$, e) $\left.\left[\mathrm{Co}^{\mathrm{II}}\right]_{\text {total }}=1.45 \mathrm{mM}, \mathrm{f}\right)\left[\mathrm{Co}^{\mathrm{II}}\right]_{\text {total }}=0.73 \mathrm{mM}$.

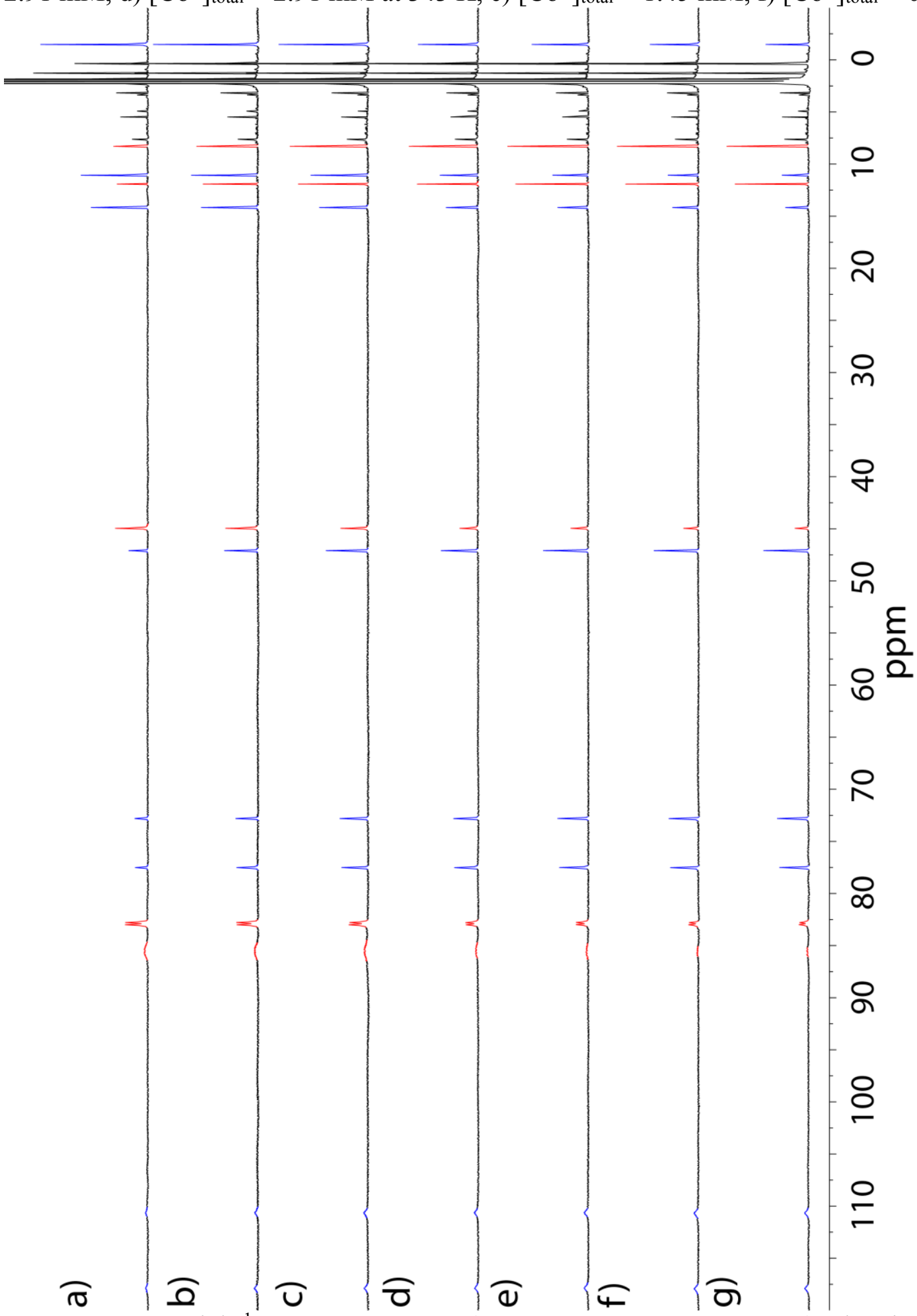

Figure S4: Partial ${ }^{1} \mathrm{H}$ NMR Spectra $\left(500 \mathrm{MHZ}, \mathrm{CD}_{3} \mathrm{CN}, 300 \mathrm{~K}\right)$ showing the slow equilibration of $\mathbf{4 c} \cdot 4 \mathrm{ClO}_{4}$ (blue) and $3 \mathbf{c} \cdot 4 \mathrm{ClO}_{4}$ (red) upon dilution from $\left[\mathrm{Co}^{\mathrm{II}}\right]_{\text {total }}=11.6 \mathrm{mM}$ to $\left[\mathrm{Co}^{\mathrm{II}}\right]_{\text {total }}=0.73 \mathrm{mM}$. a) 0 days after dilution; b) 1 day after dilution; c) 2 days after dilution; d) 3 days after dilution; e) 4 days after dilution; f) 5 days after dilution; g) 6 days after dilution. 
$\mathbf{L}^{\mathbf{d}}$

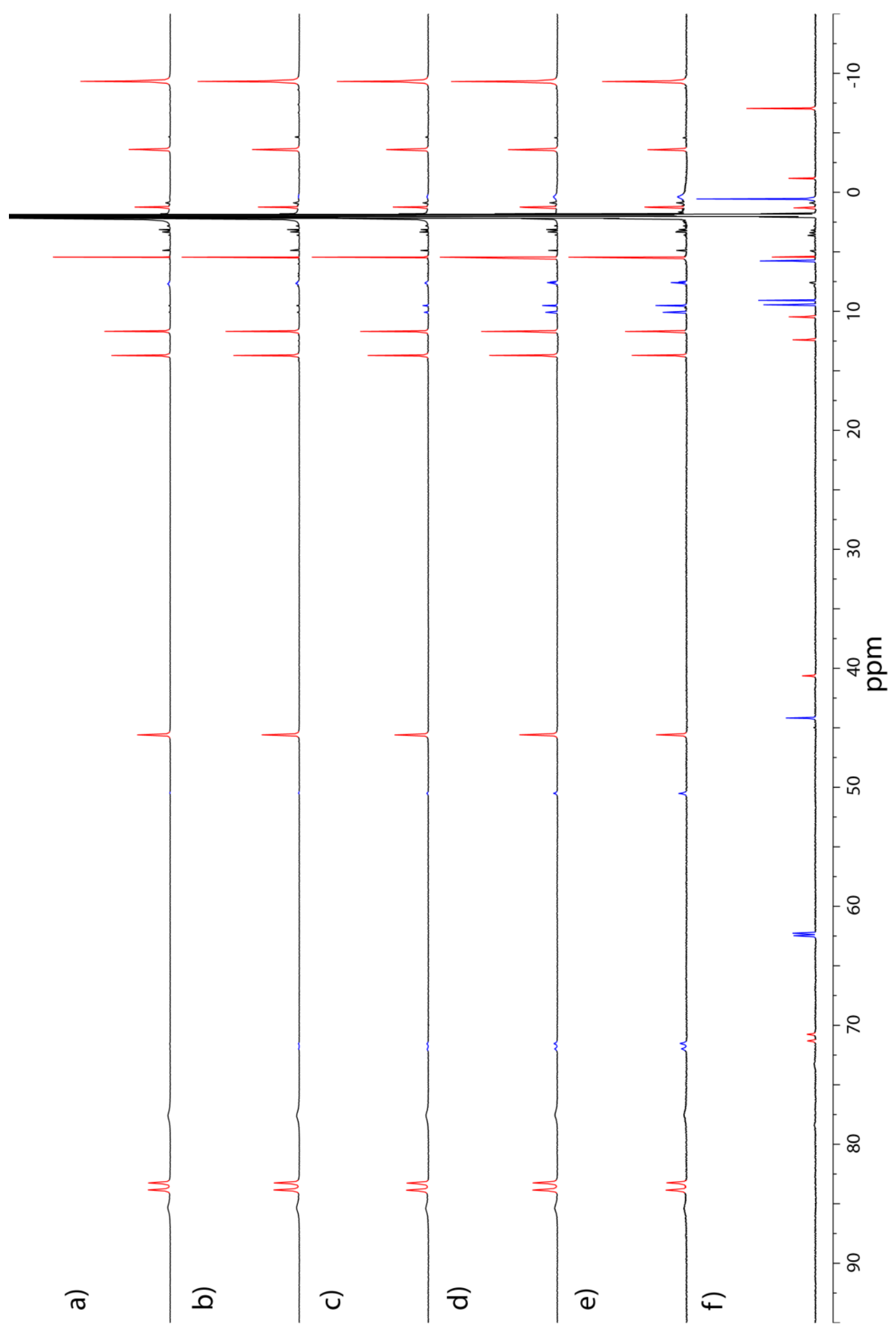

Figure S5: Partial ${ }^{1} \mathrm{H}$ NMR Spectra (500 MHZ, $\mathrm{CD}_{3} \mathrm{CN}, 300 \mathrm{~K}$ unless stated) showing the equilibrium between $\mathrm{Co}_{2}{ }_{2} \mathbf{L}_{3}{ }^{\mathbf{d}^{4+}}\left(\mathbf{4} \mathbf{d} \cdot 4 \mathrm{ClO}_{4}\right.$, blue) and $\mathrm{Co}^{\mathrm{II}}{ }_{4} \mathbf{L}^{\mathbf{d}}{ }^{8+}\left(\mathbf{3 d} \cdot 4 \mathrm{ClO}_{4}\right.$, red) as a function of $[\mathrm{Co}]_{\text {total }}$ and temperature. a) $\left[\mathrm{Co}^{\mathrm{II}}\right]_{\text {total }}=11.7 \mathrm{mM}$, b) $\left[\mathrm{Co}^{\mathrm{II}}\right]_{\text {total }}=5.84 \mathrm{mM}$, c) $\left[\mathrm{Co}^{\mathrm{II}}\right]_{\text {total }}=$ $2.92 \mathrm{mM}, \mathrm{d})\left[\mathrm{Co}^{\mathrm{II}}\right]_{\text {total }}=1.46 \mathrm{mM}$, e) $\left.\left[\mathrm{Co}^{\mathrm{II}}\right]_{\text {total }}=0.73 \mathrm{mM}, \mathrm{f}\right)\left[\mathrm{Co}^{\mathrm{II}}\right]_{\text {total }}=0.73 \mathrm{mM}$ at $343 \mathrm{~K}$. 


\section{Oxidation rate studies}

Oxidation rate studies were carried out at different concentrations for $\mathbf{L}^{\mathbf{a}-\mathbf{d}}$ as a consequence of the different inherent thermodynamic preference for helicate and tetrahedron species (Table S1). In each case, a $\left[\mathrm{Co}^{\mathrm{II}}\right]_{\text {total }}$ was utilised that would give an approximate starting equimolar ratio (Table S1). In a typical experiment, an acetonitrile solution of cerium ammonium nitrate $(11.7 \mathrm{mM})$ that contained a total of 1.5 equivalents per Co was added at a constant rate via syringe pump to the briskly stirred $\mathrm{Co}^{\mathrm{II}}$ tetrahedron-helicate equilibrium in acetonitrile at room temperature, except in the instance of the "instant" addition (" $<5 \mathrm{~s}$ ") which was done "by hand" to a similar stirred solution. The products were isolated as for the standard synthesis, by filtration onto celite, washing with water and then elution with 2:1 wateracetonitrile. Counteranion exchange with ammonium hexafluorophosphate (120 equivalents) gave the $\mathrm{PF}_{6}$ products in a typical $80 \%$ isolated yield. Product ratios were obtained by ${ }^{1} \mathrm{H}$ NMR integration (see Figures S6-8).

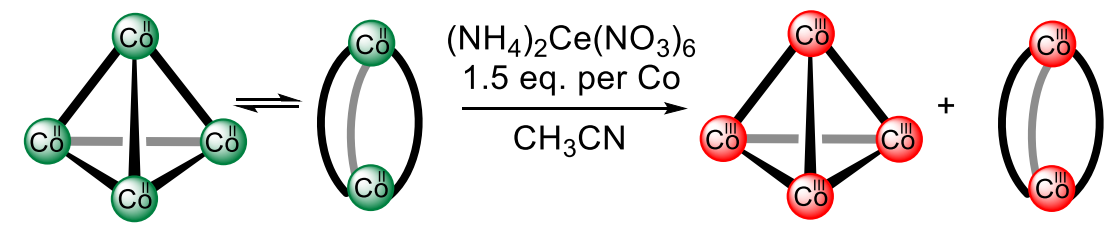

\begin{tabular}{|l|l|l|l|l|l|l|l|}
\hline \hline & $\begin{array}{l}\text { Initial }[\mathrm{Co}]_{\text {total }} \\
/ \mathrm{mM}\end{array}$ & $\begin{array}{l}\text { Co }{ }^{\text {II } 3 a-c: 4 a-} \\
\mathbf{c} \\
\text { mol ratio }\end{array}$ & \multicolumn{4}{l|}{$\begin{array}{l}\text { Fixed Co } \\
\text { (tol 1a-c:2a-c mol ratio }\end{array}$} \\
\hline \hline $\mathbf{L}^{\mathbf{a}}$ & 5.56 & $55: 45$ & $\begin{array}{l}51: 49 \\
(<5 \mathrm{~s})\end{array}$ & - & $\begin{array}{l}87: 13 \\
(22 \mathrm{~min})\end{array}$ & $\begin{array}{l}95: 5 \\
(110 \mathrm{~min})\end{array}$ & $\begin{array}{l}>99: 1 \\
(18 \mathrm{~h} 20 \mathrm{~min})\end{array}$ \\
\hline $\mathbf{L}^{\mathbf{b}}$ & 0.96 & $56: 44$ & $\begin{array}{l}51: 49 \\
(<5 \mathrm{~s})\end{array}$ & $\begin{array}{l}69: 31 \\
(8 \mathrm{~min})\end{array}$ & $\begin{array}{l}74: 26 \\
(15 \mathrm{~min})\end{array}$ & $\begin{array}{l}89: 11 \\
(74 \mathrm{~min})\end{array}$ & $\begin{array}{l}>99: 1 \\
(12 \mathrm{~h} 20 \mathrm{~min})\end{array}$ \\
\hline $\mathbf{L}^{\mathbf{c}}$ & 1.94 & $59: 41$ & $\begin{array}{l}64: 36 \\
(<5 \mathrm{~s})\end{array}$ & $\begin{array}{l}64: 36 \\
(10 \mathrm{~min})\end{array}$ & $\begin{array}{l}64: 36 \\
(19 \mathrm{~min})\end{array}$ & $\begin{array}{l}68: 32 \\
(94 \mathrm{~min})\end{array}$ & $\begin{array}{l}70: 30 \\
(15 \mathrm{~h} 40 \mathrm{~min})\end{array}$ \\
\hline
\end{tabular}

Table S1. Effect of rate of oxidant addition on the 1a-c:2a-c product ratio. 


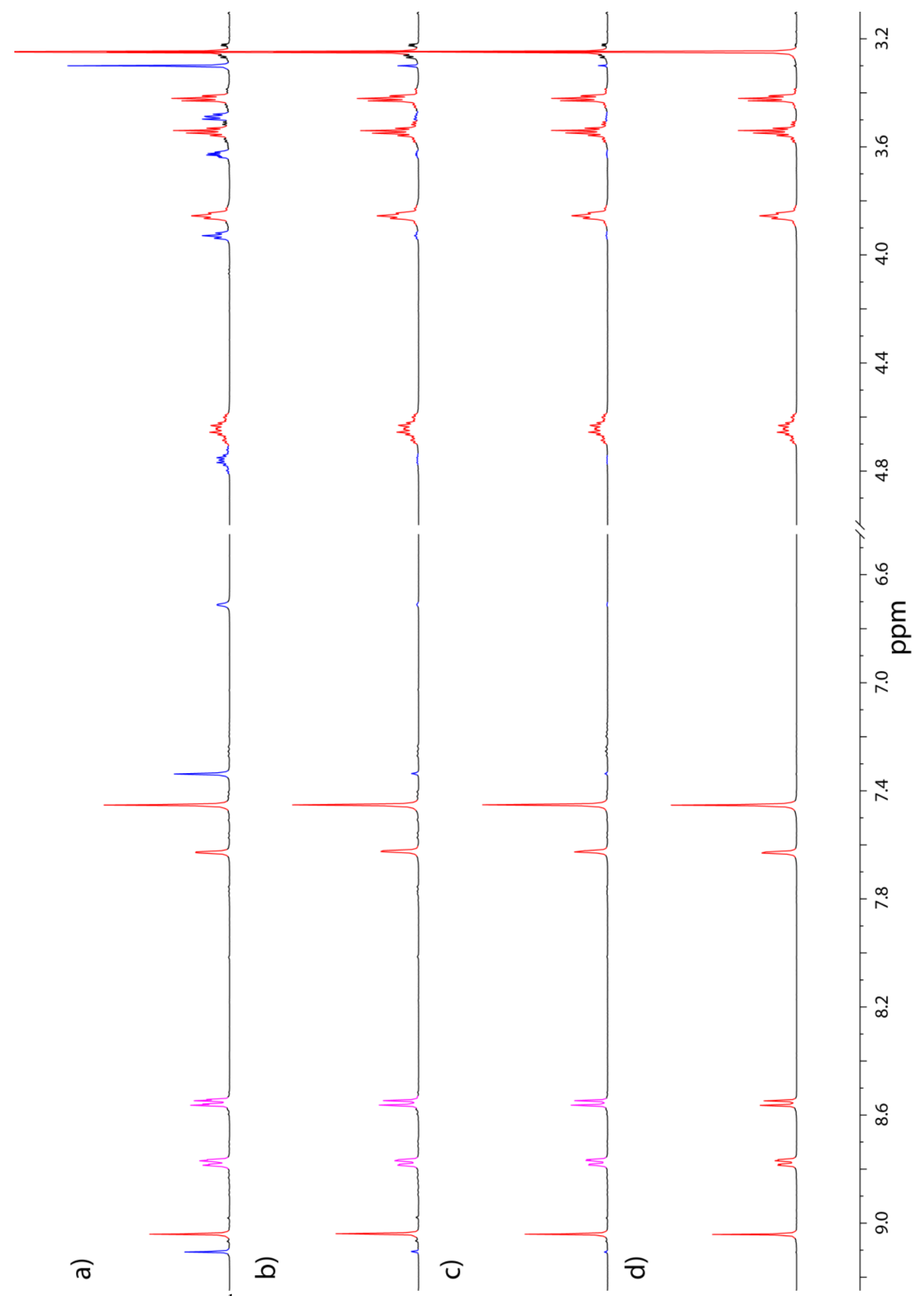

Figure S6: Partial ${ }^{1} \mathrm{H}$ NMR Spectra $\left(500 \mathrm{MHZ}, \mathrm{CD}_{3} \mathrm{CN}\right)$ of the $\mathrm{PF}_{6}$ products obtained from the variable rate of oxidant addition experiments with $\mathbf{L}^{\mathbf{a}}\left(\mathbf{1} \mathbf{a} \cdot 12 \mathrm{PF}_{6}\right.$, red; $\mathbf{2 a} \cdot 6 \mathrm{PF}_{6}$, blue $)$ at an initial $\left[\mathrm{Co}^{\mathrm{II}}\right]_{\text {total }}=5.84 \mathrm{mM}$. a) Addition time $=<5 \mathrm{~s}, 51 \mathrm{~mol} \% \mathbf{1 a} \cdot 12 \mathrm{PF}_{6}$; b) Addition time $=$ $22 \mathrm{~min}, 87 \mathrm{~mol} \% \mathbf{1 a} \cdot 12 \mathrm{PF}_{6}$; c) Addition time $=110 \mathrm{~min}, 95 \mathrm{~mol} \% \mathbf{1 a} \cdot 12 \mathrm{PF}_{6}$; d) Addition time $=18 \mathrm{~h} 20 \mathrm{~min}, 99 \mathrm{~mol} \% \mathbf{1 a} \cdot 12 \mathrm{PF}_{6}$. 
$\mathbf{L}^{\mathbf{b}}$

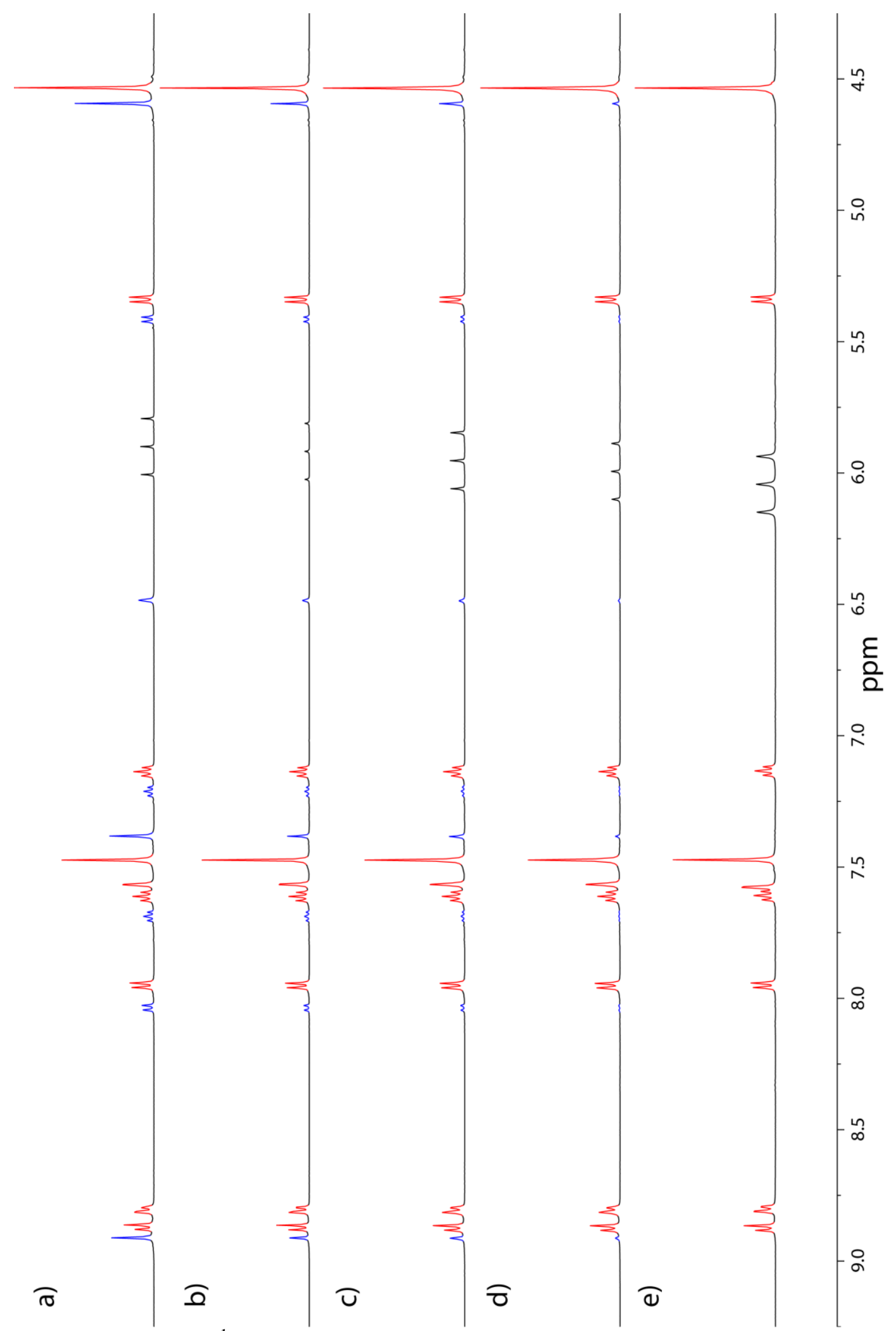

Figure S7: Partial ${ }^{1} \mathrm{H}$ NMR Spectra $\left(500 \mathrm{MHZ}, \mathrm{CD}_{3} \mathrm{CN}\right)$ of the $\mathrm{PF}_{6}$ products obtained from the variable rate of oxidant addition experiments with $\mathbf{L}^{\mathbf{b}}\left(\mathbf{1} \mathbf{b} \cdot 12 \mathrm{PF}_{6}\right.$, red; $\mathbf{2 b} \cdot 6 \mathrm{PF}_{6}$, blue $)$ at an initial $\left[\mathrm{Co}^{\mathrm{II}}\right]_{\text {total }}=1.45 \mathrm{mM}$. a) Addition time $=<5 \mathrm{~s}, 51 \mathrm{~mol} \% \mathbf{1 b} \cdot 12 \mathrm{PF}_{6}$; b) Addition time $=$ $\left.8 \mathrm{~min}, 69 \mathrm{~mol} \% \mathbf{1 b} \cdot 12 \mathrm{PF}_{6} ; \mathrm{c}\right)$ Addition time $=15 \mathrm{~min}, 74 \mathrm{~mol} \% \mathbf{1 b} \cdot 12 \mathrm{PF}_{6} ;$ d) Addition time $=74 \mathrm{~min}, 89 \mathrm{~mol} \% \mathbf{1 b} \cdot 12 \mathrm{PF}_{6}$; e) Addition time $=12 \mathrm{~h}, 20 \mathrm{~min}, 99 \mathrm{~mol} \% \mathbf{1 b} \cdot 12 \mathrm{PF}_{6}$. 
$\mathbf{L}^{\mathbf{c}}$

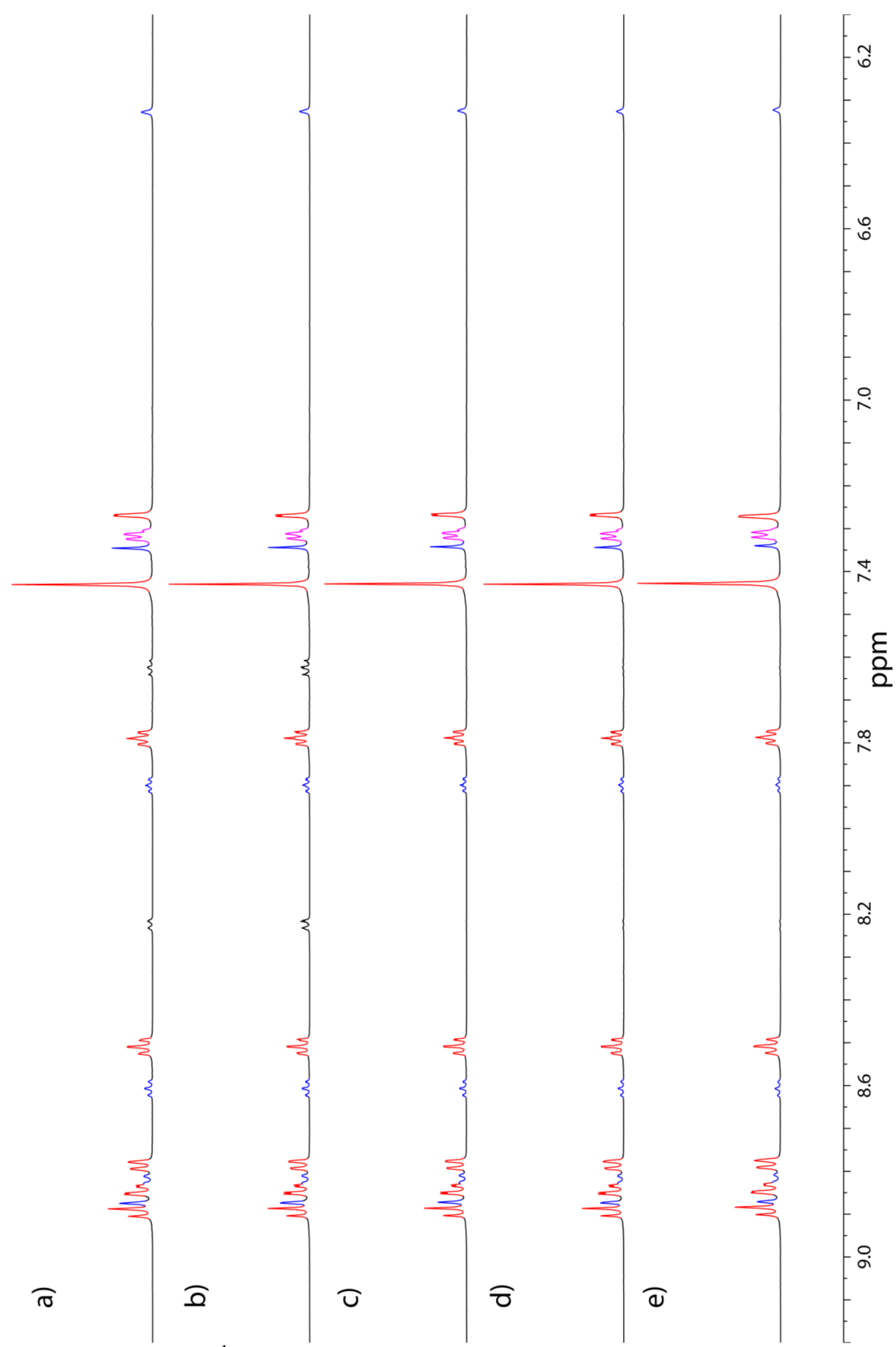

Figure S8: Partial ${ }^{1} \mathrm{H}$ NMR Spectra (500 MHZ, $\mathrm{CD}_{3} \mathrm{CN}$ ) of the $\mathrm{PF}_{6}$ products obtained from the variable rate of oxidant addition experiments with $\mathbf{L}^{\mathbf{c}}\left(\mathbf{1} \mathbf{c} \cdot 12 \mathrm{PF}_{6}\right.$, red; $\mathbf{2} \mathbf{c} \cdot 6 \mathrm{PF}_{6}$, blue) at an initial $\left[\mathrm{Co}^{\mathrm{II}}\right]_{\text {total }}=2.90 \mathrm{mM}$. a) Addition time $=<5 \mathrm{~s}, 64 \mathrm{~mol} \% 1 \mathrm{c} \cdot 12 \mathrm{PF}_{6}$; b) Addition time $=$ $10 \mathrm{~min}, 64 \mathrm{~mol} \% \mathbf{1 c} \cdot 12 \mathrm{PF}_{6}$; c) Addition time $=19 \mathrm{~min}, 64 \mathrm{~mol} \% \mathbf{1 c} \cdot 12 \mathrm{PF}_{6}$; d) Addition time $\left.=94 \mathrm{~min}, 68 \mathrm{~mol} \% \mathbf{1 c} \cdot 12 \mathrm{PF}_{6} ; \mathrm{e}\right)$ Addition time $=15 \mathrm{~h} 40 \mathrm{~min}, 70 \mathrm{~mol} \% \mathbf{1 c} \cdot 12 \mathrm{PF}_{6}$. 


\section{Helicate stability experiments}

Solutions of helicates 2a-d in $\mathrm{CD}_{3} \mathrm{CN}(\sim 2 \mathrm{mM})$ were monitored by ${ }^{1} \mathrm{H}$ NMR spectroscopy as a function of time and temperature.

$\mathbf{2 a}$

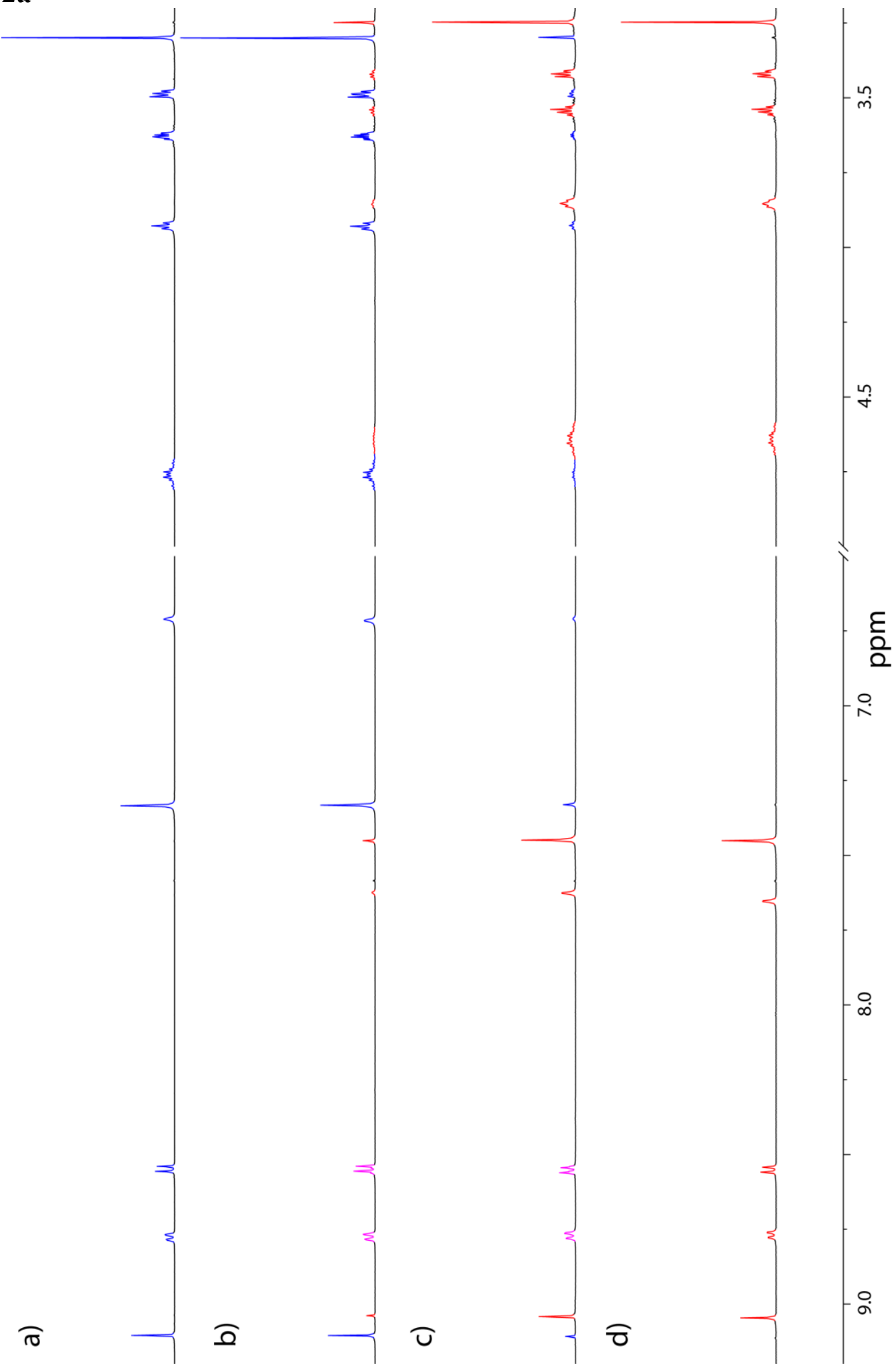

Figure S9: ${ }^{1} \mathrm{H}$ NMR Spectra $\left(500 \mathrm{MHZ}, \mathrm{CD}_{3} \mathrm{CN}\right)$ of $2 \mathrm{a} \cdot 6 \mathrm{PF}_{6}(1.75 \mathrm{mM})$ after a) 1 week at $\mathrm{RT}$; and b) then after heating for 1 week at $40{ }^{\circ} \mathrm{C}$; and c) then after heating for 1 week at 50 ${ }^{\circ} \mathrm{C}$; and d) then after heating for 2 days at $60^{\circ} \mathrm{C}$. [colour code: $\mathbf{2 a}$, blue; $\mathbf{1 a}$, red] 
2b

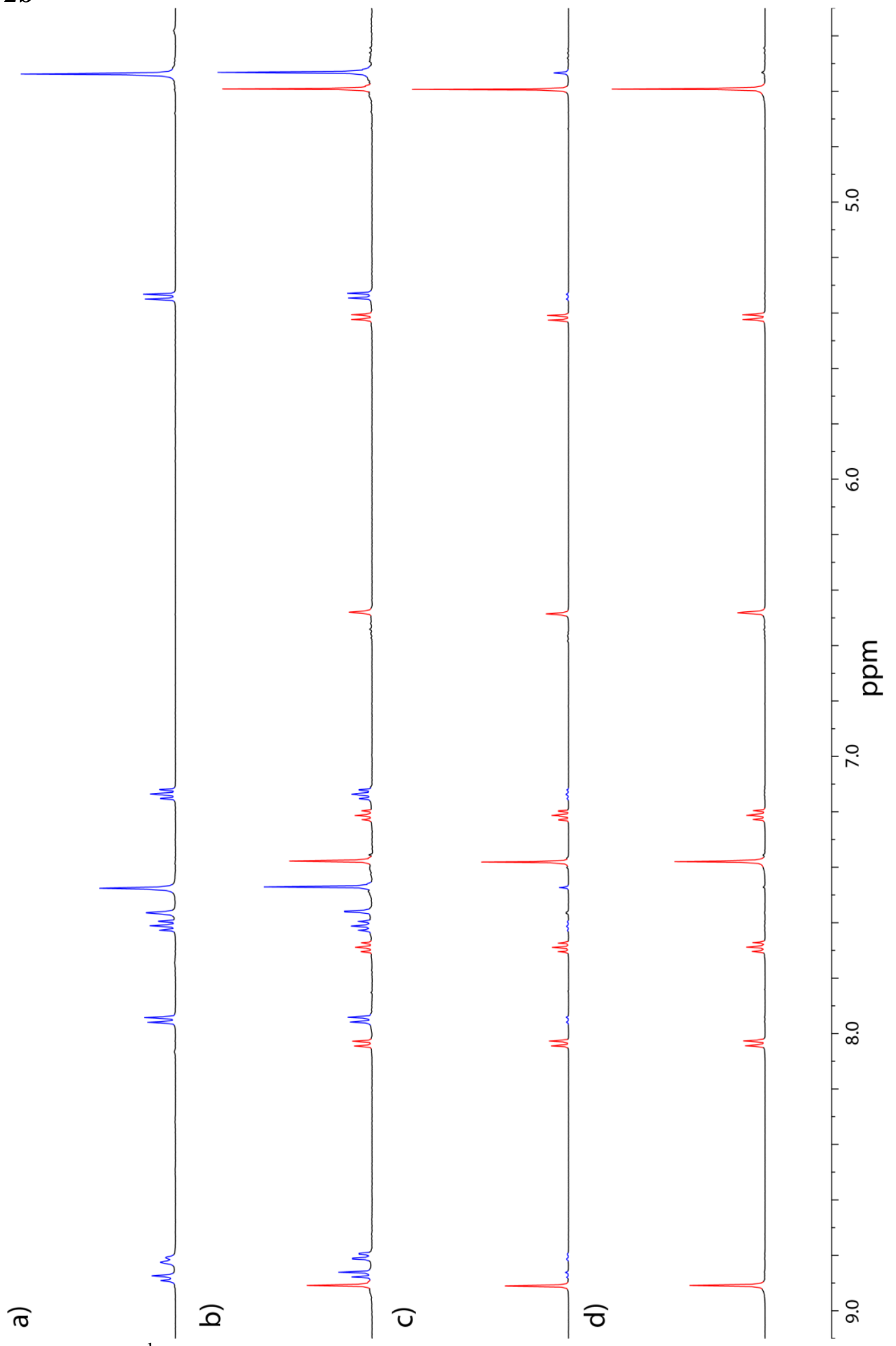

Figure S10: ${ }^{1} \mathrm{H}$ NMR Spectra $\left(500 \mathrm{MHZ}, \mathrm{CD}_{3} \mathrm{CN}\right)$ of $\mathbf{2 b} \cdot 6 \mathrm{PF}_{6}(2.14 \mathrm{mM})$ after a) 1 week at RT; and b) then after heating for 1 week at $40^{\circ} \mathrm{C}$; and c) then after heating for 1 week at 50 ${ }^{\circ} \mathrm{C}$; and d) then after heating for 6 days at $60{ }^{\circ} \mathrm{C}$. [colour code: $\mathbf{2 b}$, blue; $\mathbf{1 b}$, red] 


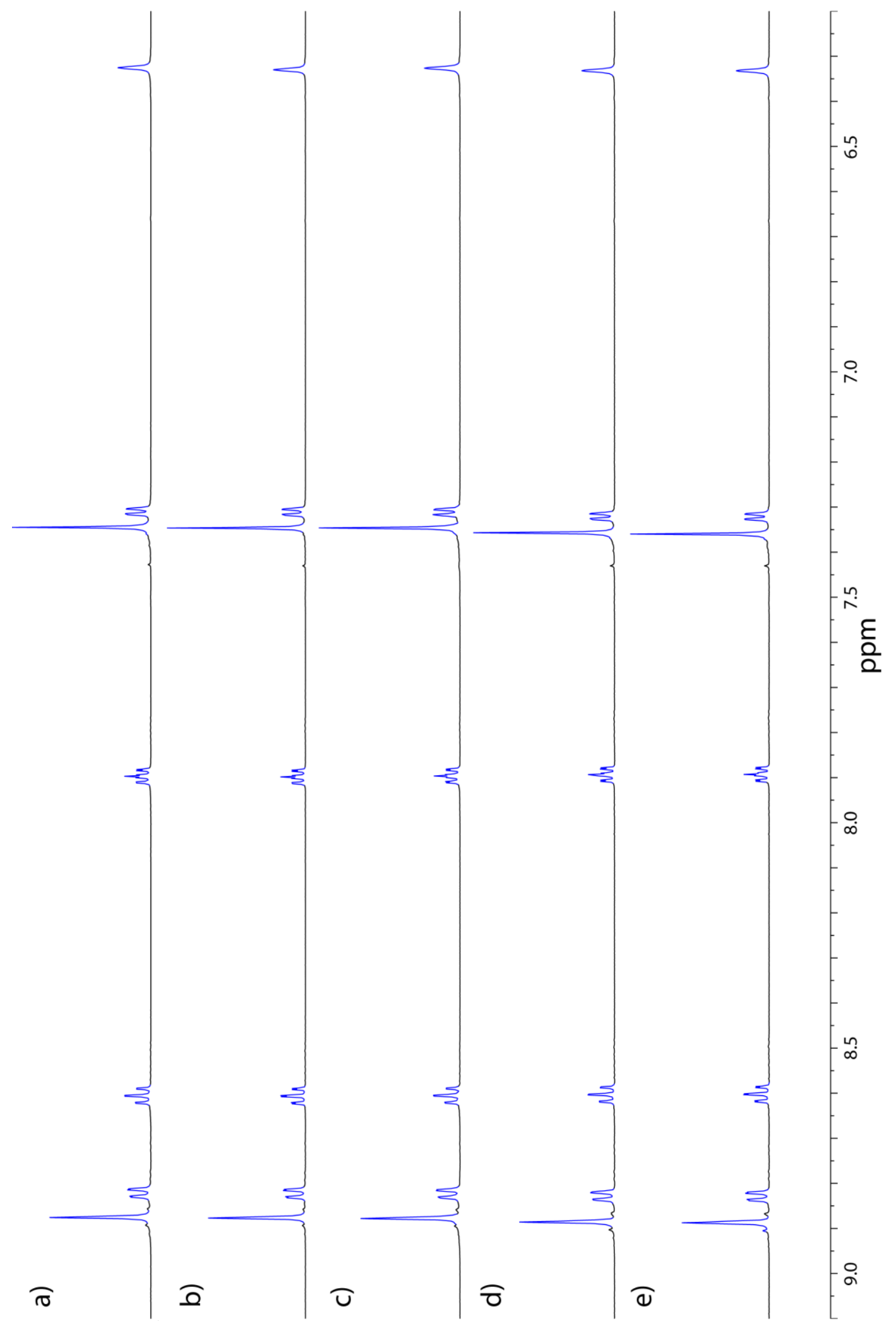

Figure S11: ${ }^{1} \mathrm{H}$ NMR Spectra $\left(500 \mathrm{MHZ}, \mathrm{CD}_{3} \mathrm{CN}\right)$ of $2 \mathrm{c} \cdot 6 \mathrm{PF}_{6}(2.03 \mathrm{mM})$ after a) 1 week at RT; and b) then after heating for 1 week at $40{ }^{\circ} \mathrm{C}$; and c) then after heating for 1 week at 50 ${ }^{\circ} \mathrm{C}$; and d) then after heating for 1 week at $60{ }^{\circ} \mathrm{C}$; and e) then after heating for 1 week at 70 ${ }^{\circ} \mathrm{C}$. [colour code: $\mathbf{2 c}$, blue] 

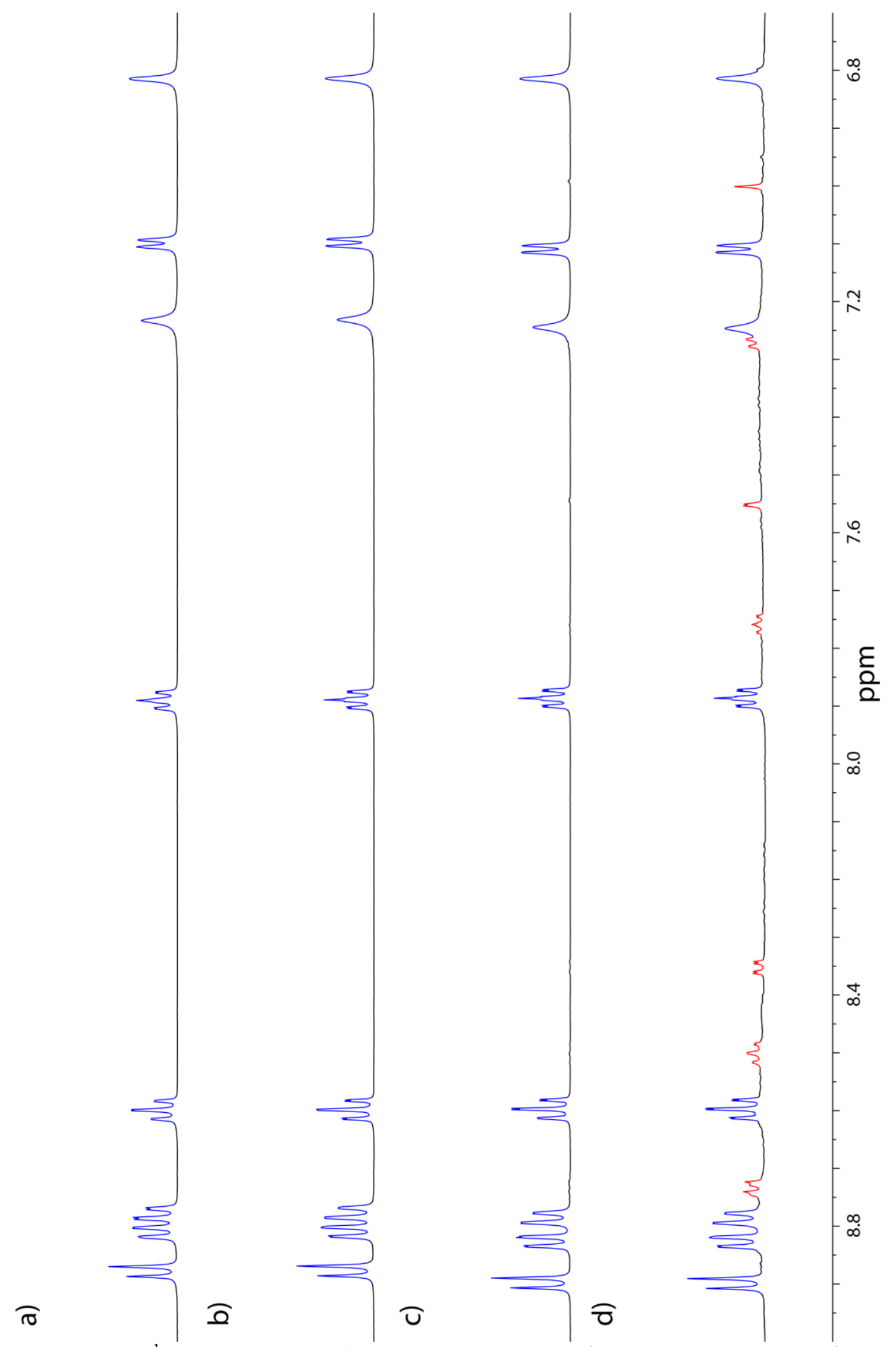

Figure S12: ${ }^{1} \mathrm{H}$ NMR Spectra (500 MHZ, $\left.\mathrm{CD}_{3} \mathrm{CN}\right)$ of $\mathbf{2 d} \cdot 6 \mathrm{PF}_{6}(2.82 \mathrm{mM})$ after a) 1 week at $\mathrm{RT}$; and b) then after heating for 1 week at $50{ }^{\circ} \mathrm{C}$; and c) then after heating for 1 week at 60 ${ }^{\circ} \mathrm{C}$; and d) then after heating for 1 week at $70{ }^{\circ} \mathrm{C}$. [colour code: $2 \mathbf{d}$, blue; 1d, red] 


\section{Switching protocols}

\section{Helicate to Tetrahedron}

\section{Representative example for $2 \mathrm{~b}$ to $1 \mathrm{~b}$}

To a solution of $\mathbf{2 b} \cdot 6 \mathrm{PF}_{6}(0.0044 \mathrm{~g}, 1.78 \mu \mathrm{mol})$ in $\mathrm{CD}_{3} \mathrm{CN}(0.60 \mathrm{~mL})$, a solution of tetrabutylammonium iodide $(0.0013 \mathrm{~g}, 3.74 \mu \mathrm{mol})$ in $\mathrm{CD}_{3} \mathrm{CN}(0.06 \mathrm{~mL})$ was added, with ${ }^{1} \mathrm{H}$ NMR spectroscopy showing the formation of paramagnetic species (Figure S13b). Over time, the remaining $\mathrm{Co}^{\mathrm{II}}$ helicate (show in blue) diminished the system reaching equilibrium after 3 days (Figure S13c). Cerium ammonium nitrate $(0.0030 \mathrm{~g}, 5.40 \mu \mathrm{mol})$ in acetonitrile $(0.28$ $\mathrm{mL}$ ) was added slowly over $4 \mathrm{~h} 40 \mathrm{~min}$ resulting in precipitation of an orange solid, which was filtered onto celite and then washed with acetonitrile. This solid was eluted with a mixture of water-acetonitrile $(2: 1,5.0 \mathrm{~mL})$, to which was added an aqueous saturated ammonium hexafluorophosphate solution. The resulting precipitate was filtered onto celite, washed with water and then eluted with acetonitrile. Removal of the solvent under reduced pressure gave $\mathbf{1 b} \cdot 12 \mathrm{PF}_{6}$ (Figure S13d).

a)
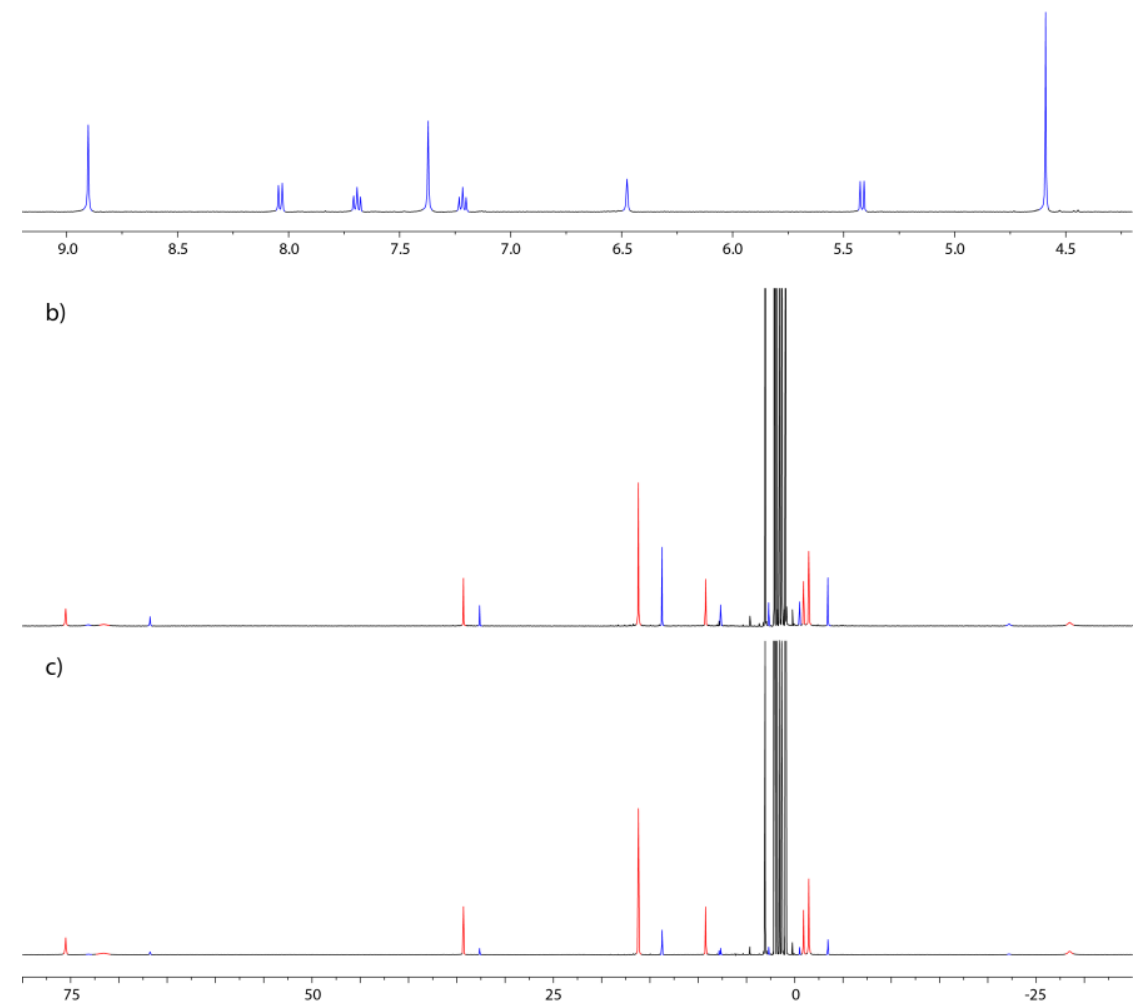

d)

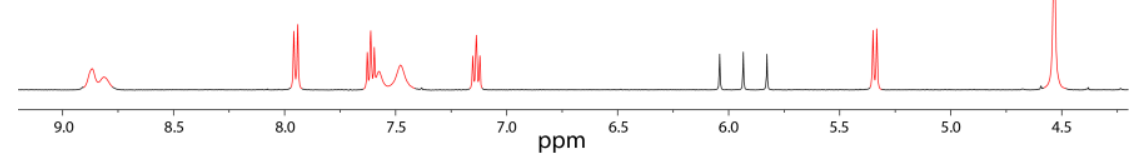

Figure S13: Partial ${ }^{1} \mathrm{H}$ NMR Spectra (500 MHZ, $\mathrm{CD}_{3} \mathrm{CN}, 300 \mathrm{~K}$ ) of a) $\mathbf{2 b} \cdot 6 \mathrm{PF}_{6}$; and b) after the addition of TBAI; and c) then after 3 days at RT; and d) the product isolated after the slow re-oxidation with CAN (1b·12 $\left.\mathrm{PF}_{6}\right)$. [colour code: blue, helicates; red, tetrahedra] 

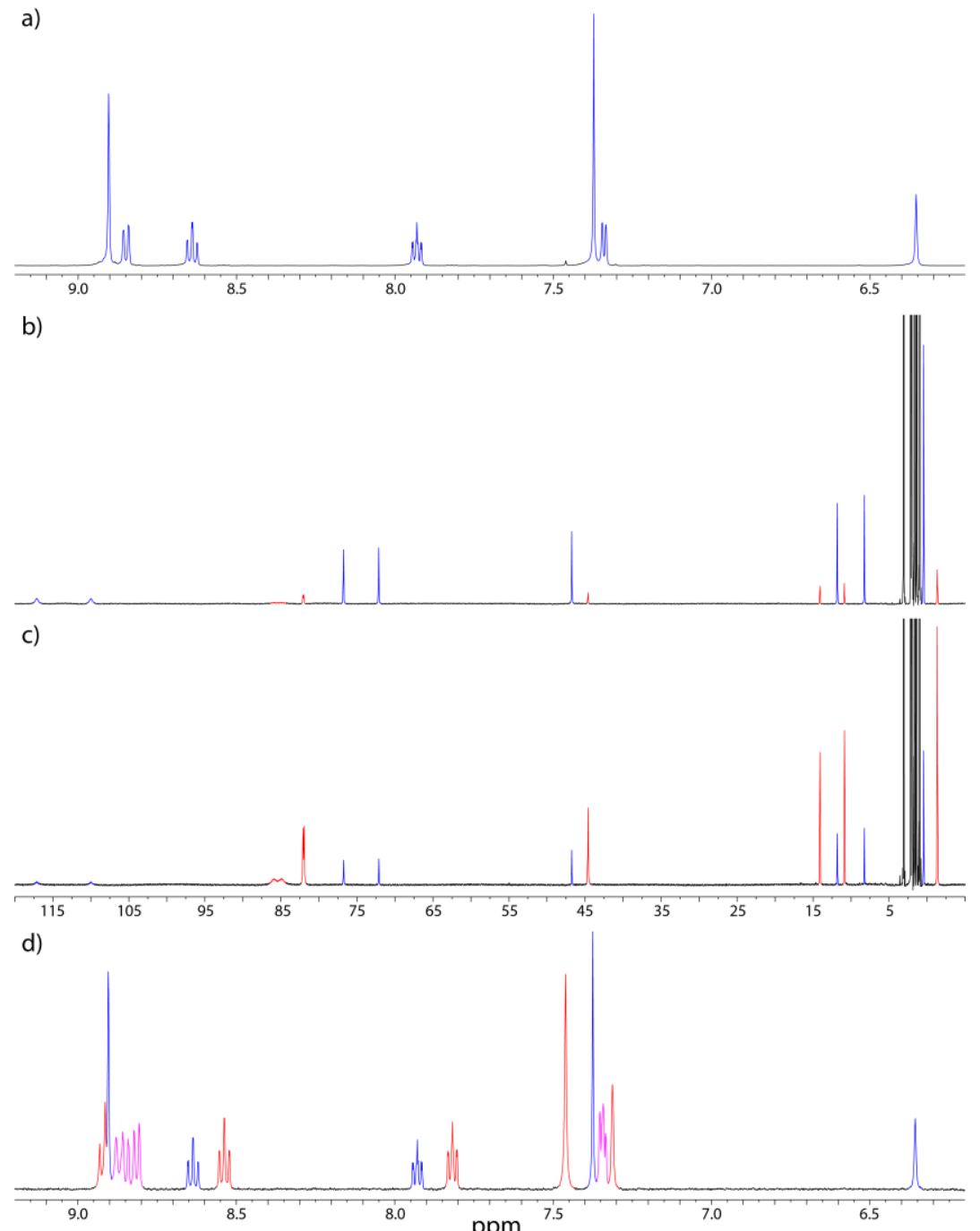

Figure S14: Partial ${ }^{1} \mathrm{H}$ NMR Spectra (500 MHZ, $\mathrm{CD}_{3} \mathrm{CN}, 300 \mathrm{~K}$ ) of a) $2 \mathrm{c} \cdot 6 \mathrm{PF}_{6}$; and b) after the addition of TBAI; and c) then after 5 days at RT; and d) the product isolated after the slow reoxidation with $\mathrm{CAN}\left(\mathbf{1} \mathbf{c} \cdot 12 \mathrm{PF}_{6}\right)$. [colour code: blue, helicates; red, tetrahedra] 


\section{Tetrahedron to Helicate}

\section{Representative example for $1 \mathrm{~b}$ to $2 \mathrm{~b}$}

To a solution of $\mathbf{1 b} \cdot 12 \mathrm{PF}_{6}(0.0019 \mathrm{~g}, 0.390 \mu \mathrm{mol})$ in $\mathrm{CD}_{3} \mathrm{CN}(0.55 \mathrm{~mL})$, a solution of tetrabutylammonium iodide $(0.0006 \mathrm{~g}, 1.66 \mu \mathrm{mol})$ in $\mathrm{CD}_{3} \mathrm{CN}(0.17 \mathrm{~mL})$ was added, with the ${ }^{1} \mathrm{H}$ NMR spectrum showing the formation of paramagnetic species (Figure S15b) A subsequent ${ }^{1} \mathrm{H}$ NMR spectrum recorded at $343 \mathrm{~K}$ (Figure S15c) indicated a predominant conversion to $\mathrm{Co}^{\mathrm{II}}$ helicate. The rapid addition of cerium ammonium nitrate $(0.0013 \mathrm{~g}, 2.36$ $\mu \mathrm{mol})$ in acetonitrile $(0.48 \mathrm{~mL})$ at this temperature resulted in the precipitation of an orange solid, which was filtered onto celite and washed with acetonitrile. The orange solid was eluted with a mixture of water-acetonitrile $(2: 1,5.0 \mathrm{~mL})$, to which was added an aqueous saturated ammonium hexafluorophosphate solution. The resulting precipitate was filtered onto celite, washed with water and then eluted with acetonitrile. Removal of the solvent under reduced pressure gave $\mathbf{2 b} \cdot 6 \mathrm{PF}_{6}$ (Figure $\mathrm{S} 15 \mathrm{~d}$ ).
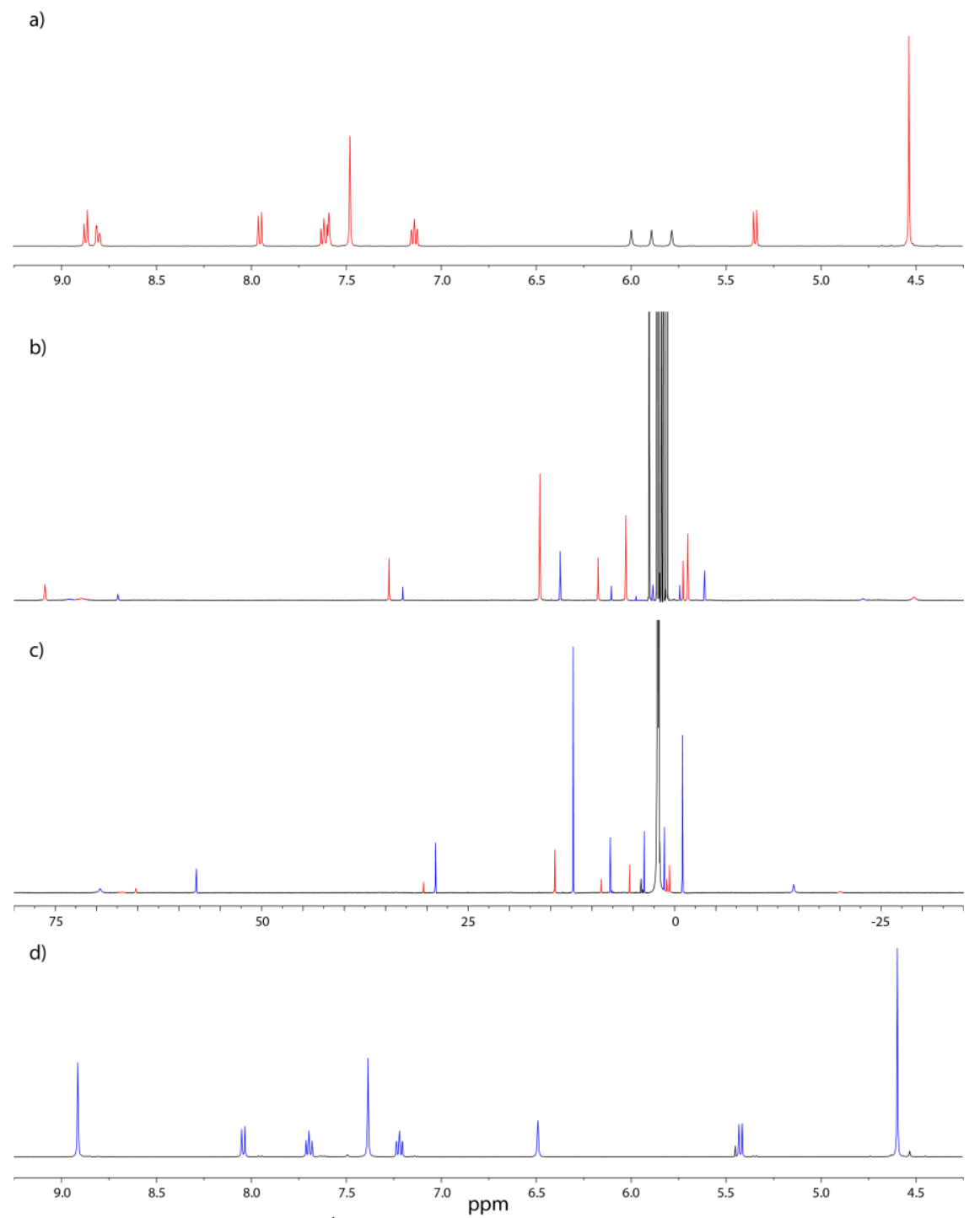

Figure S15: Partial ${ }^{1} \mathrm{H}$ NMR Spectra (500 MHZ, $\mathrm{CD}_{3} \mathrm{CN}, 300 \mathrm{~K}$ unless stated) of a) $\mathbf{1 b} \cdot 12 \mathrm{PF}_{6}$; and $\mathrm{b}$ ) after the addition of TBAI at $300 \mathrm{~K}$; and c) then recorded at $343 \mathrm{~K}$; and d) the product isolated after the re-oxidation with CAN $\left(\mathbf{2 b} \cdot 6 \mathrm{PF}_{6}\right)$. [colour code: blue, helicates; red, tetrahedra] 


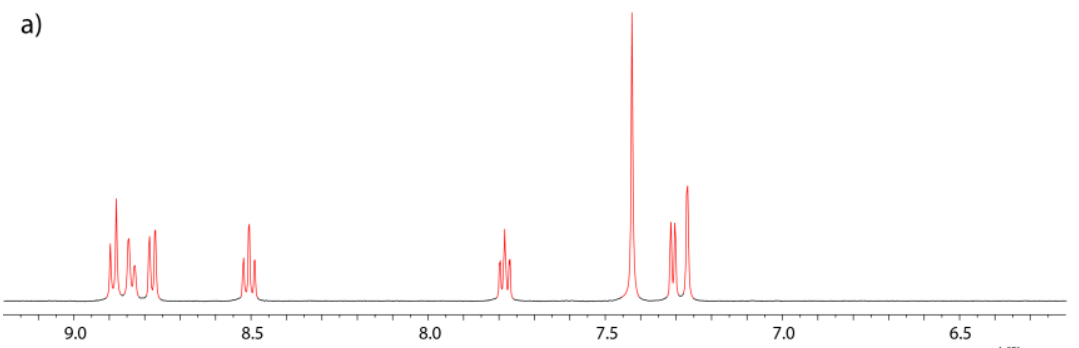

b)

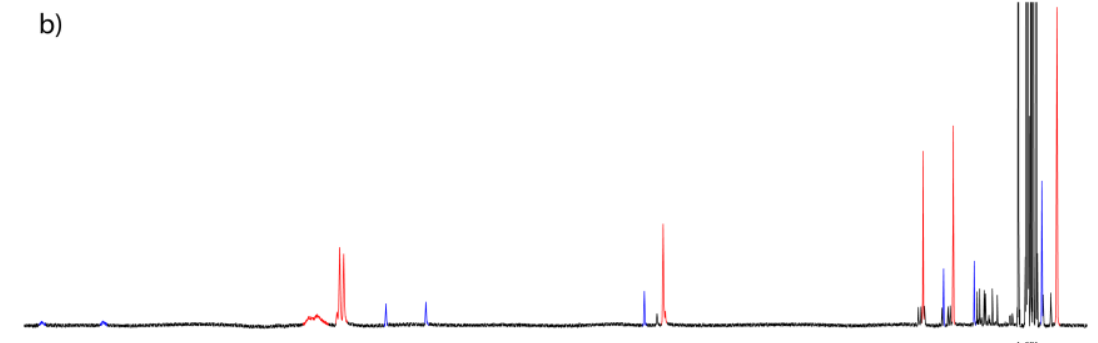

c)
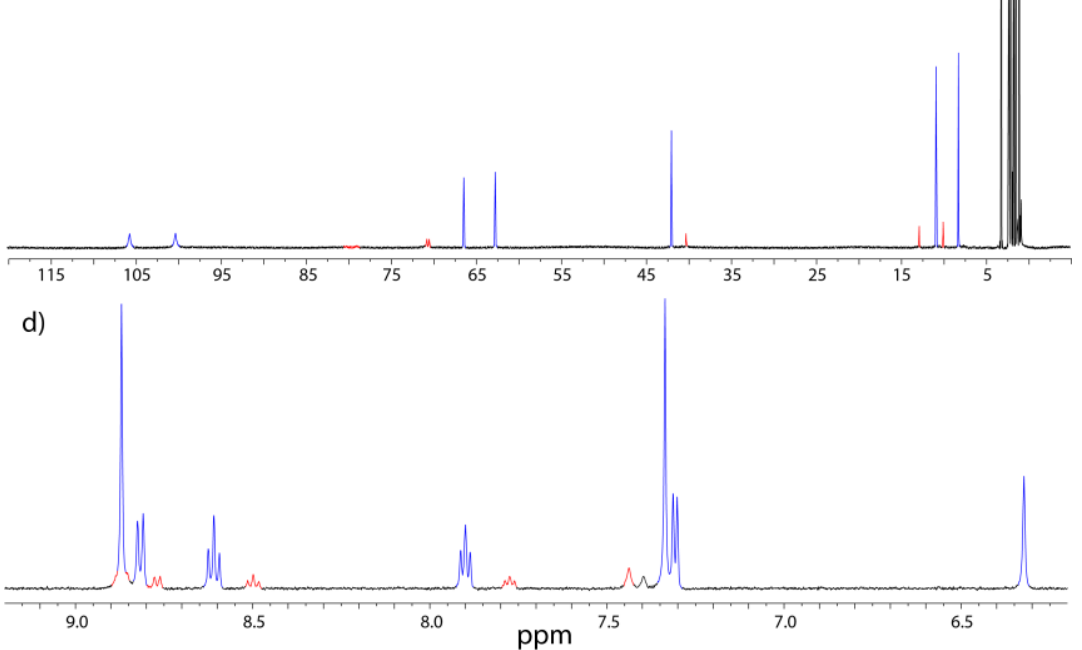

Figure S16: Partial ${ }^{1} \mathrm{H}$ NMR Spectra (500 MHZ, $\mathrm{CD}_{3} \mathrm{CN}, 300 \mathrm{~K}$ unless stated) of a) 1c $12 \mathrm{PF}_{6}$; and b) after the addition of TBAI at $300 \mathrm{~K}$; and c) then recorded at $343 \mathrm{~K}$; and d) the product isolated after the re-oxidation with CAN $\left(\mathbf{2} \mathbf{c} \cdot 6 \mathrm{PF}_{6}\right)$. [colour code: blue, helicates; red, tetrahedra] 


\section{Photoredox induced rearrangement}

To a solution of $2 \mathrm{a} \cdot 6 \mathrm{PF}_{6}(0.0009 \mathrm{~g}, 0.333 \mu \mathrm{mol})$ in $\mathrm{CD}_{3} \mathrm{CN}(0.60 \mathrm{~mL})$, a solution of $\operatorname{Ir}(\text { ppy })_{2}(\mathrm{dtbbpy})\left(\mathrm{PF}_{6}\right)(0.0003 \mathrm{~g}, 0.334 \mu \mathrm{mol})$ in $\mathrm{CD}_{3} \mathrm{CN}(0.11 \mathrm{~mL})$ was added. The solution was degassed by sparging with argon and then a light shone on the sample for 2 days with NMR spectra recorded at regular timed intervals. The conversion to $\mathbf{1 a} \cdot 12 \mathrm{PF}_{6}$ is shown in Fig. S17. A similar blank reaction was set up in a darkened NMR tube shown in Fig. S18.

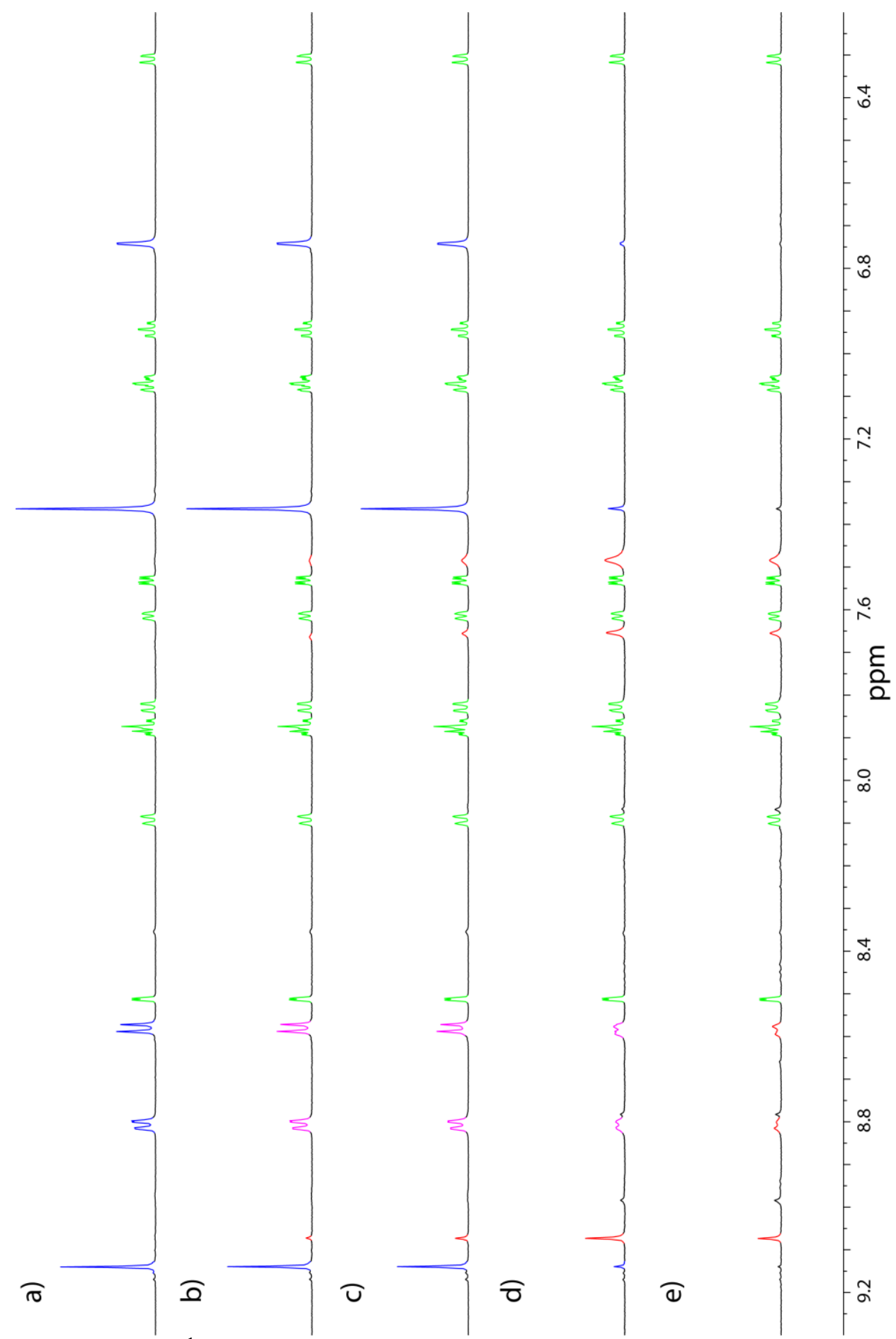

Figure S17: ${ }^{1} \mathrm{H}$ NMR Spectra (500 $\mathrm{MHZ}, \mathrm{CD}_{3} \mathrm{CN}$ ) of $2 \mathrm{a} \cdot 6 \mathrm{PF}_{6}$ (shown in blue) in the presence of $\operatorname{Ir}(\mathrm{ppy})_{2}(\mathrm{dtbbpy})\left(\mathrm{PF}_{6}\right)$ (shown in green) upon light irradiation as a function of time. a) $0 \mathrm{~h}$; b) $2 \mathrm{~h}$; c) $7 \mathrm{~h}$; d) $21 \mathrm{~h}$; e) $35 \mathrm{~h}$. Signals for $1 \mathbf{a} \cdot 12 \mathrm{PF}_{6}$ that appear upon exposure to light are shown in red. 


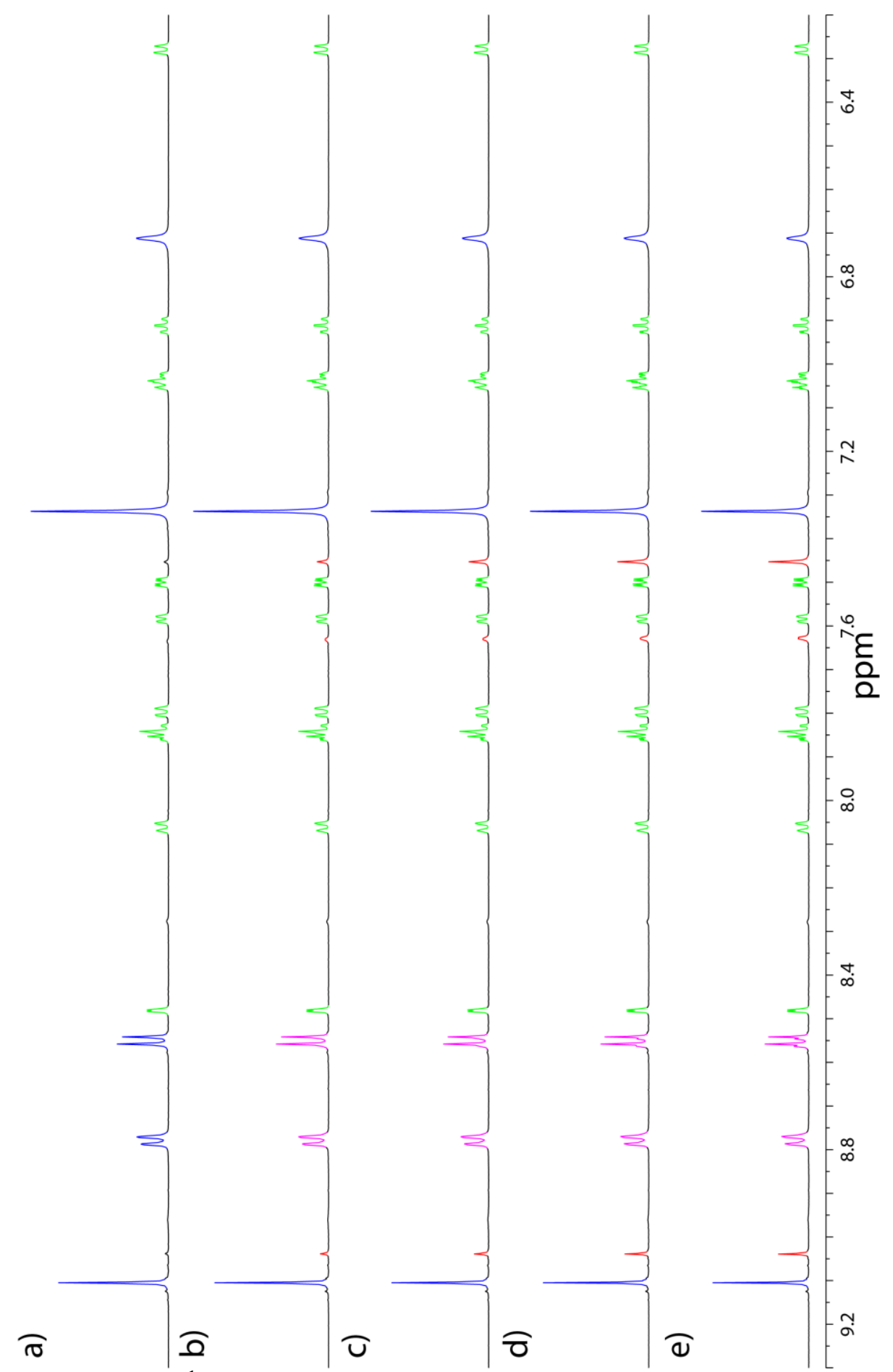

Figure S18: ${ }^{1} \mathrm{H}$ NMR Spectra $\left(500 \mathrm{MHZ}, \mathrm{CD}_{3} \mathrm{CN}\right.$ ) of $2 \mathbf{a}^{2} \cdot 6 \mathrm{PF}_{6}$ (shown in blue) in the presence of $\operatorname{Ir}(\mathrm{ppy})_{2}(\mathrm{dtbbpy})\left(\mathrm{PF}_{6}\right)$ (shown in green) in a darkened NMR tube as a function of time. a) $0 \mathrm{~h}$; b) $1 \mathrm{~d}$; c) $3 \mathrm{~d}$; d) 4 d; e) $5 \mathrm{~d}$. Signals for $\mathbf{1 a} \cdot 12 \mathrm{PF}_{6}$ that appear are shown in red. 
To a solution of $\mathbf{2} \mathbf{b} \cdot 6 \mathrm{PF}_{6}(0.0009 \mathrm{~g}, 0.373 \mu \mathrm{mol})$ in $\mathrm{CD}_{3} \mathrm{CN}(0.60 \mathrm{~mL})$, a solution of $\operatorname{Ir}(\text { ppy })_{2}(\mathrm{dtbbpy})\left(\mathrm{PF}_{6}\right)(0.0003 \mathrm{~g}, 0.374 \mu \mathrm{mol})$ in $\mathrm{CD}_{3} \mathrm{CN}(0.12 \mathrm{~mL})$ was added. The solution was degassed by sparging with argon and then a light shone on the sample for 4 days with NMR spectra recorded at regular timed intervals. The conversion to $\mathbf{1 b} \cdot 12 \mathrm{PF}_{6}$ is shown in Fig. S19. A similar blank reaction was set up in a darkened NMR tube shown in Fig. S20.

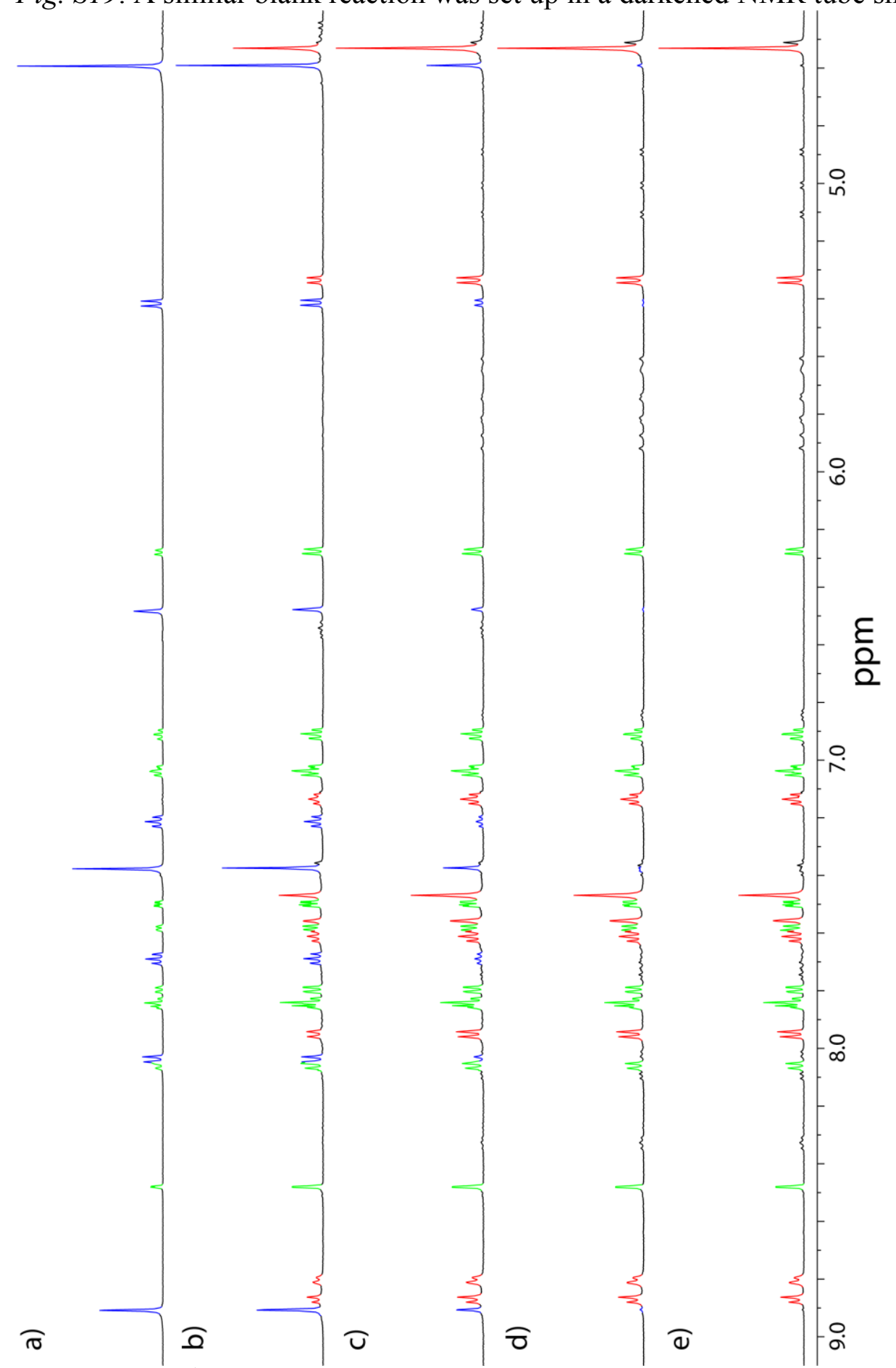

Figure S19: ${ }^{1} \mathrm{H}$ NMR Spectra $\left(500 \mathrm{MHZ}, \mathrm{CD}_{3} \mathrm{CN}\right)$ of $\mathbf{2 b} \cdot 6 \mathrm{PF}_{6}$ (shown in blue) in the presence of $\operatorname{Ir}(\mathrm{ppy})_{2}(\mathrm{dtbbpy})\left(\mathrm{PF}_{6}\right)$ (shown in green) upon light irradiation as a function of time. a) $0 \mathrm{~h}$; b) $1 \mathrm{~d}$; c) $2 \mathrm{~d}$; d) $3 \mathrm{~d}$; e) $4 \mathrm{~d}$. Signals for $\mathbf{1 b} \cdot 12 \mathrm{PF}_{6}$ that appear upon exposure to light are shown in red. 


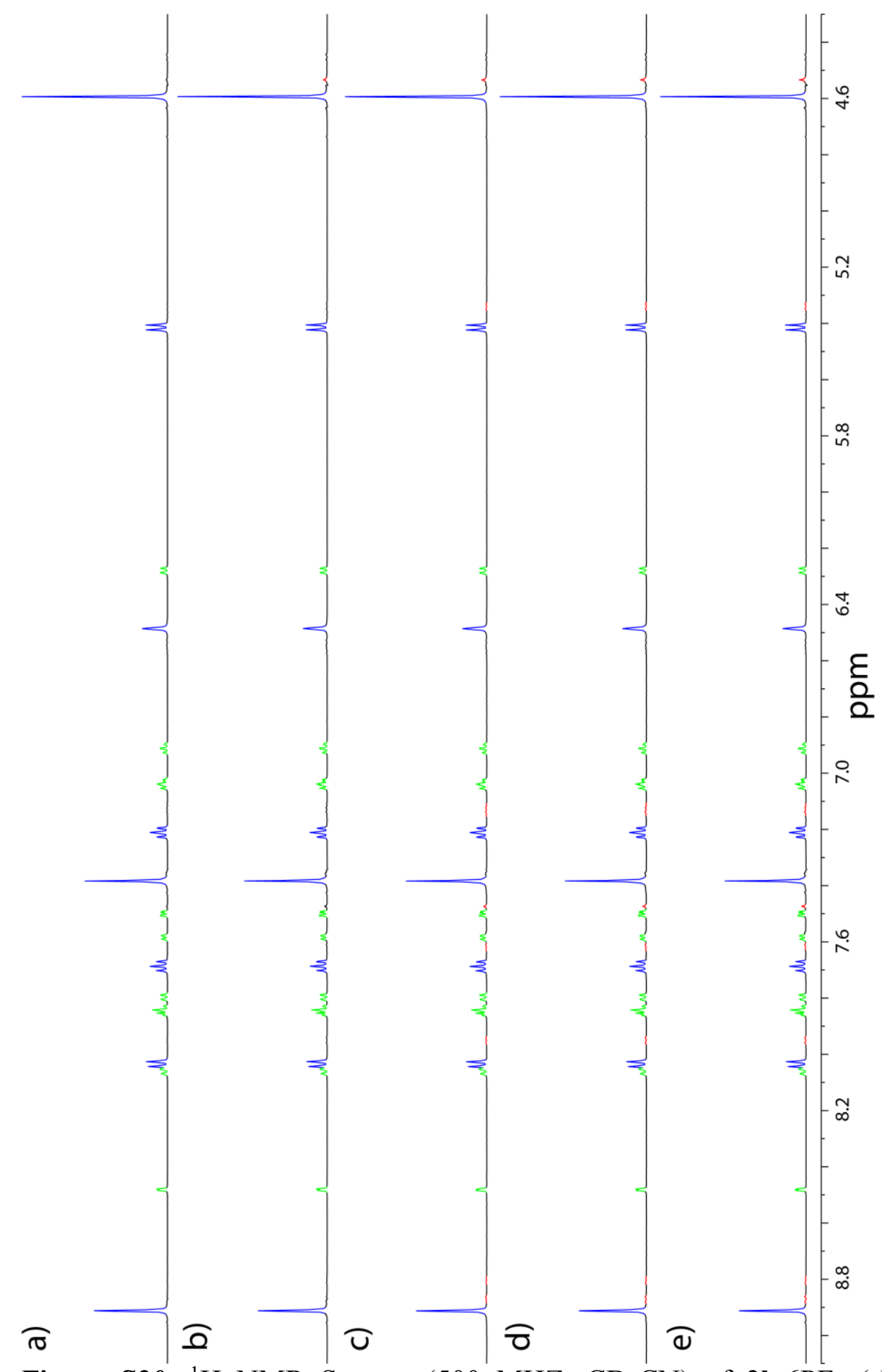

Figure S20: ${ }^{1} \mathrm{H}$ NMR Spectra (500 $\mathrm{MHZ}, \mathrm{CD}_{3} \mathrm{CN}$ ) of $\mathbf{2 b} \cdot 6 \mathrm{PF}_{6}$ (shown in blue) in the presence of $\operatorname{Ir}(\mathrm{ppy})_{2}(\mathrm{dtbbpy})\left(\mathrm{PF}_{6}\right)$ (shown in green) in a darkened NMR tube as a function of time. a) $0 \mathrm{~h}$; b) 1 d; c) $3 \mathrm{~d}$; d) $5 \mathrm{~d}$; e) $7 \mathrm{~d}$. Signals for $\mathbf{1 b} \cdot 12 \mathrm{PF}_{6}$ that appear are shown in red. 
To a solution of $2 \mathrm{c} \cdot 6 \mathrm{PF}_{6}(0.0021 \mathrm{~g}, 0.978 \mu \mathrm{mol})$ in $\mathrm{CD}_{3} \mathrm{CN}(0.60 \mathrm{~mL})$, a solution of $\operatorname{Ir}(\text { ppy })_{2}(\mathrm{dtbbpy})\left(\mathrm{PF}_{6}\right)(0.0009 \mathrm{~g}, 0.979 \mu \mathrm{mol})$ in $\mathrm{CD}_{3} \mathrm{CN}(0.32 \mathrm{~mL})$ was added. The solution was degassed by sparging with argon and then a light shone on the sample for 9 days with NMR spectra recorded at regular timed intervals. The conversion to $\mathbf{1 b} \cdot 12 \mathrm{PF}_{6}$ is shown in Fig. S21.

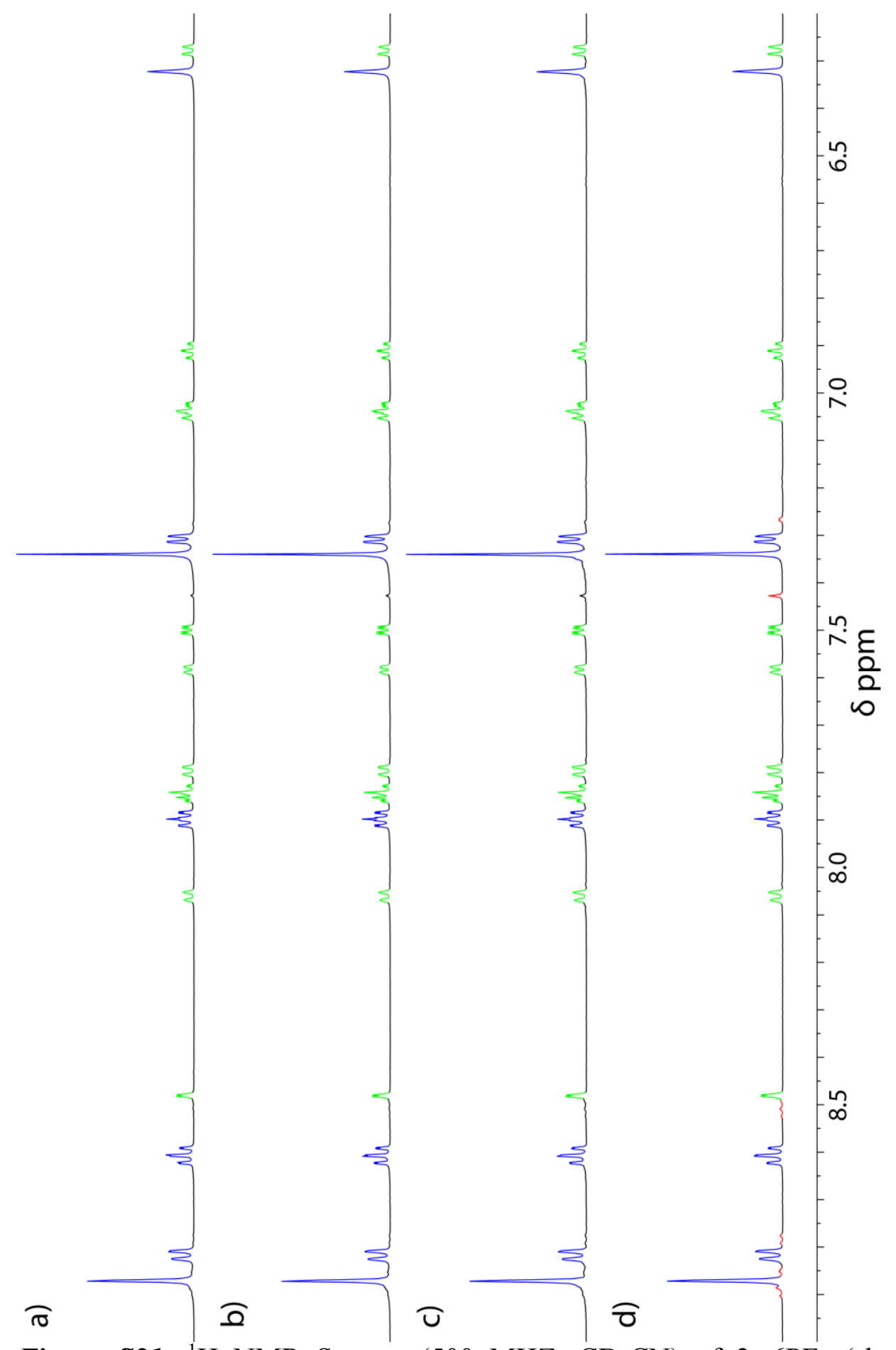

Figure S21: ${ }^{1} \mathrm{H}$ NMR Spectra (500 $\mathrm{MHZ}, \mathrm{CD}_{3} \mathrm{CN}$ ) of $2 \mathrm{c} \cdot 6 \mathrm{PF}_{6}$ (shown in blue) in the presence of $\operatorname{Ir}(\mathrm{ppy})_{2}(\mathrm{dtbbpy})\left(\mathrm{PF}_{6}\right)$ (shown in green) upon light irradiation as a function of time. a) $0 \mathrm{~h}$; b) $2 \mathrm{~d}$; c) $4 \mathrm{~d}$; d) $9 \mathrm{~d}$. Signals for $1 \mathrm{c} \cdot 12 \mathrm{PF}_{6}$ that appear upon exposure to light are shown in red. 


\section{NMR Spectroscopy of all compounds}

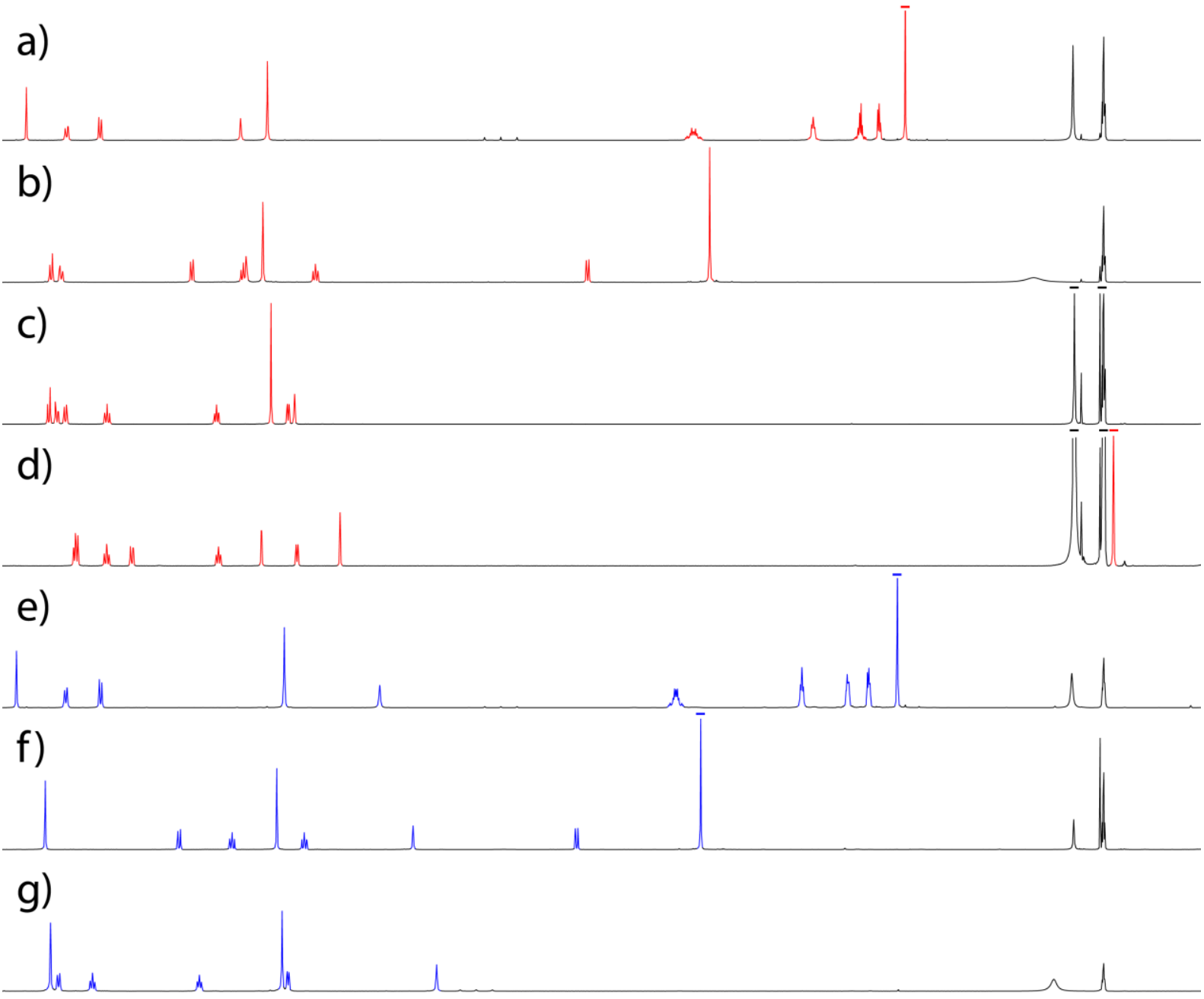

h)

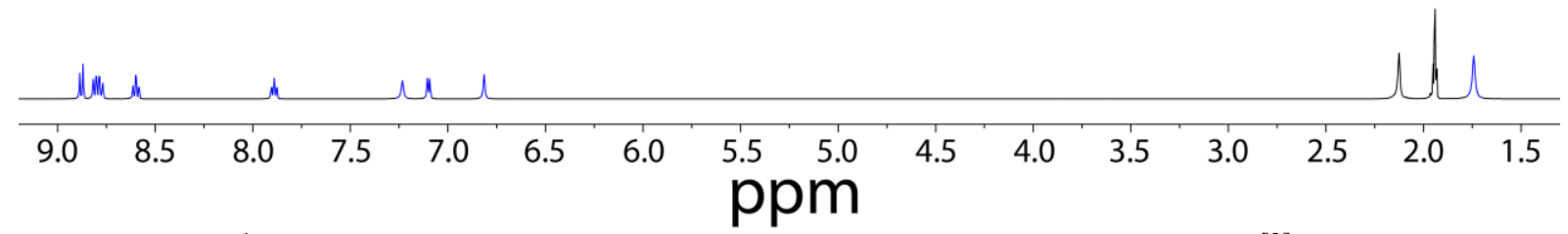

Figure S22: ${ }^{1} \mathrm{H}$ NMR spectra of all helicates and tetrahedra. a) $\left.\mathbf{1 a} \cdot 12 \mathrm{PF}_{6} ;{ }^{[2]} \mathrm{b}\right) \mathbf{1} \mathbf{b} \cdot 12 \mathrm{PF}_{6}$; c) 1c. $12 \mathrm{PF}_{6}$; d) $\mathbf{1 d} \cdot 12 \mathrm{PF}_{6}$; e) $\mathbf{2 a} \cdot 6 \mathrm{PF}_{6}$; f) $\mathbf{2 b} \cdot 6 \mathrm{PF}_{6} ;$ g) $\mathbf{2 c} \cdot 6 \mathrm{PF}_{6}$; h) $\mathbf{2 d} \cdot 6 \mathrm{PF}_{6}$. [colour code: 2a-d $6 \mathrm{PF}_{6}$, blue; $\mathbf{1 a - d} \cdot 12 \mathrm{PF}_{6}$, red] 


\section{${ }^{1}$ H NMR Spectra of Individual Compounds}

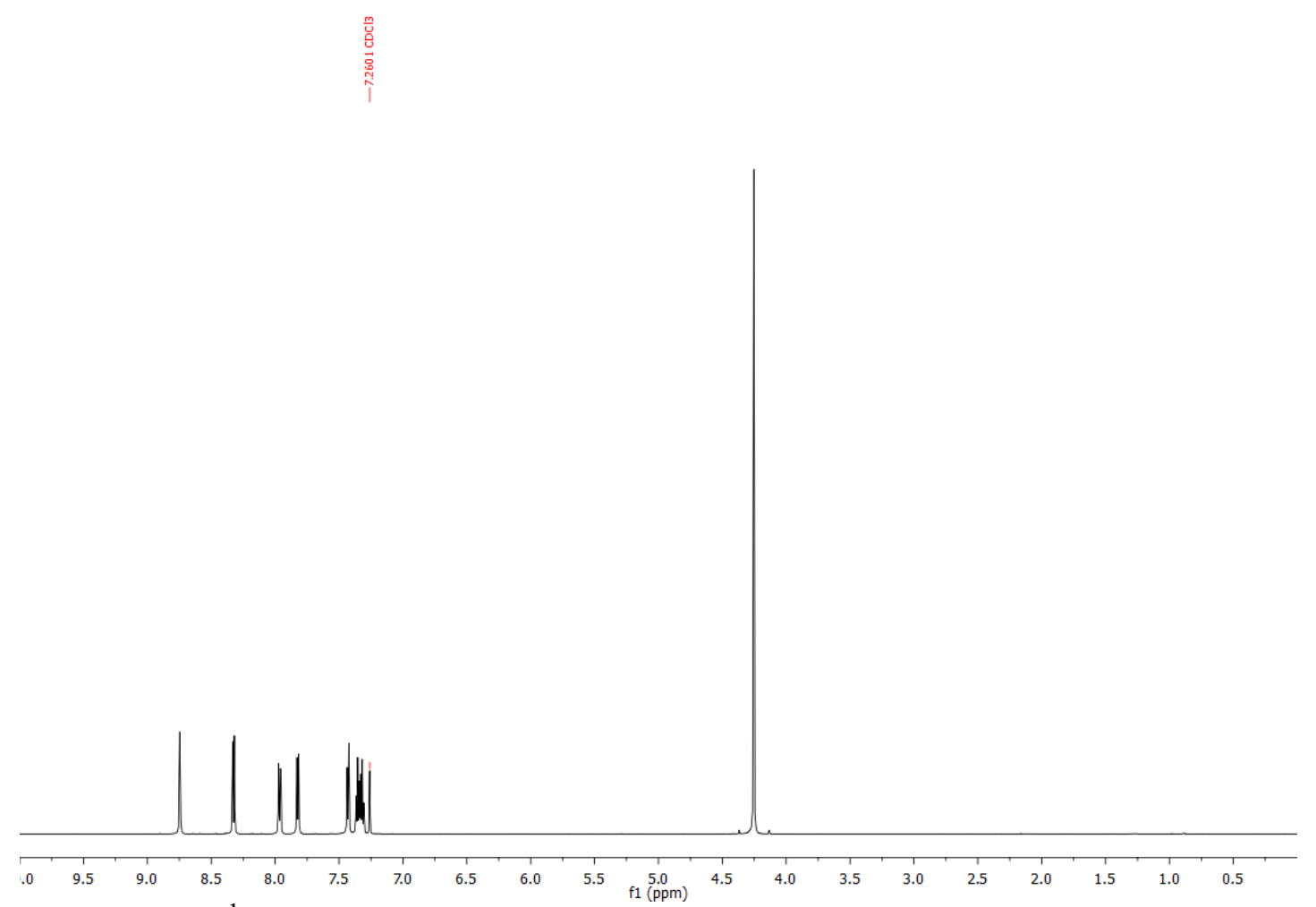

Figure S23: ${ }^{1} \mathrm{H}$ NMR Spectrum (600 $\left.\mathrm{MHZ}, \mathrm{CDCl}_{3}\right)$ of 2-(5-bromopyridin-2-yl)-1-methyl1,3-benzimidazole.

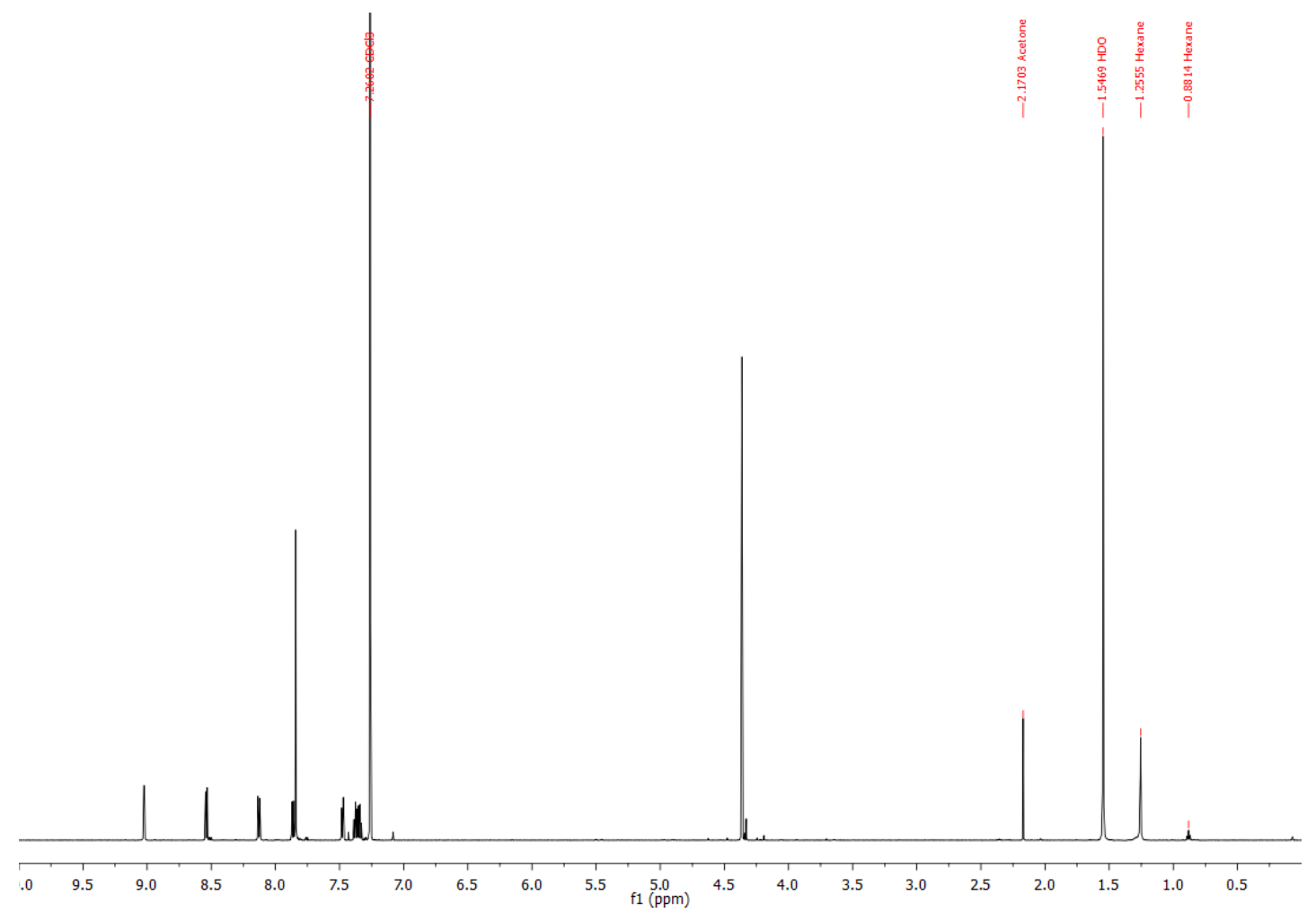

Figure S24: ${ }^{1} \mathrm{H}$ NMR Spectrum (600 $\left.\mathrm{MHZ}, \mathrm{CDCl}_{3}\right)$ of 1,4-bis(6-(1-methyl-1,3benzimidazolyl)pyridin-3-yl)benzene $\left(\mathbf{L}^{\mathbf{b}}\right)$. 


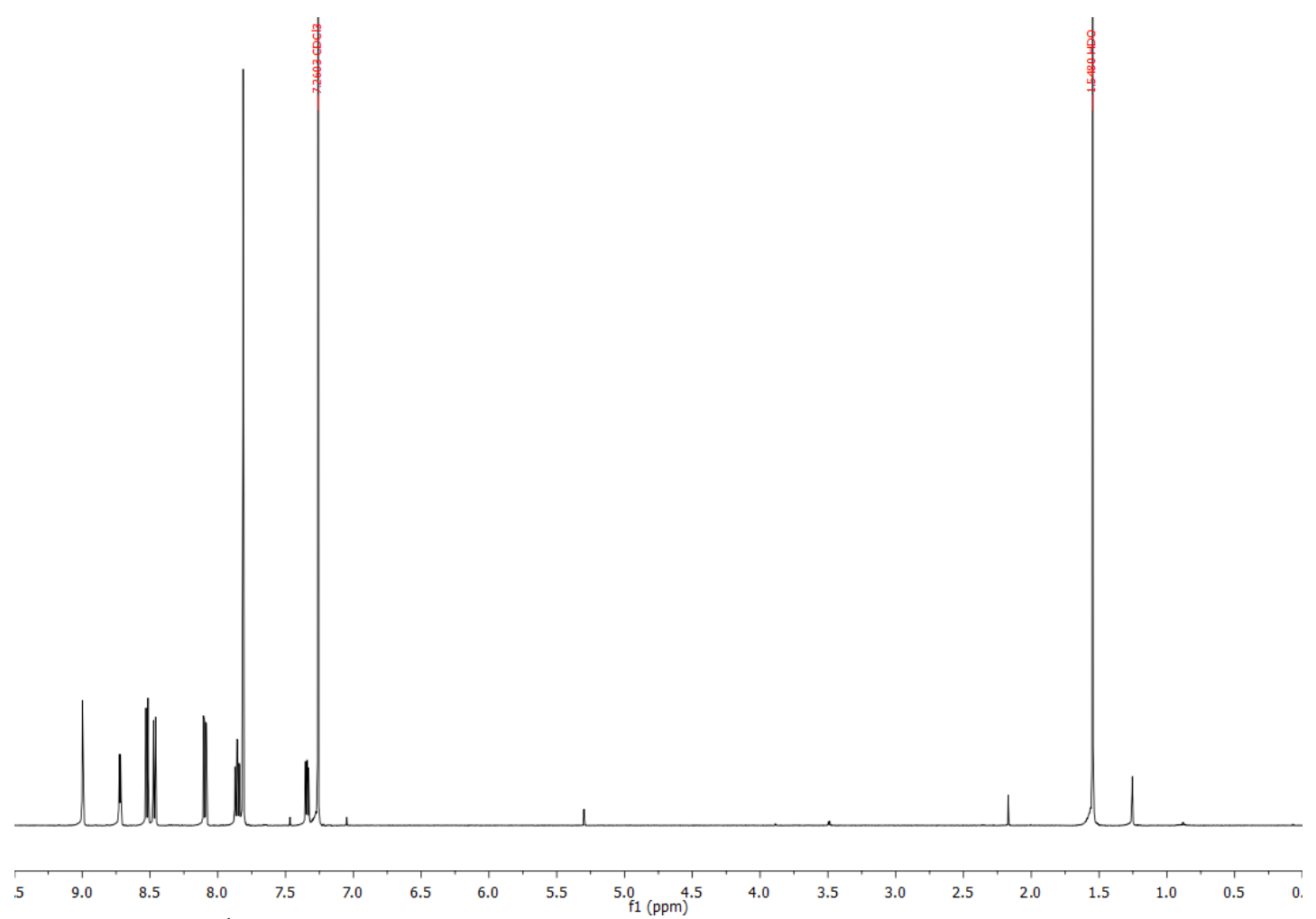

Figure S25: ${ }^{1} \mathrm{H}$ NMR Spectrum (500 $\mathrm{MHZ}, \mathrm{CDCl}_{3}$ ) of 1,4-bis(5-bipyridin-2,2'-yl)benzene $\left(\mathbf{L}^{\mathbf{c}}\right)$.

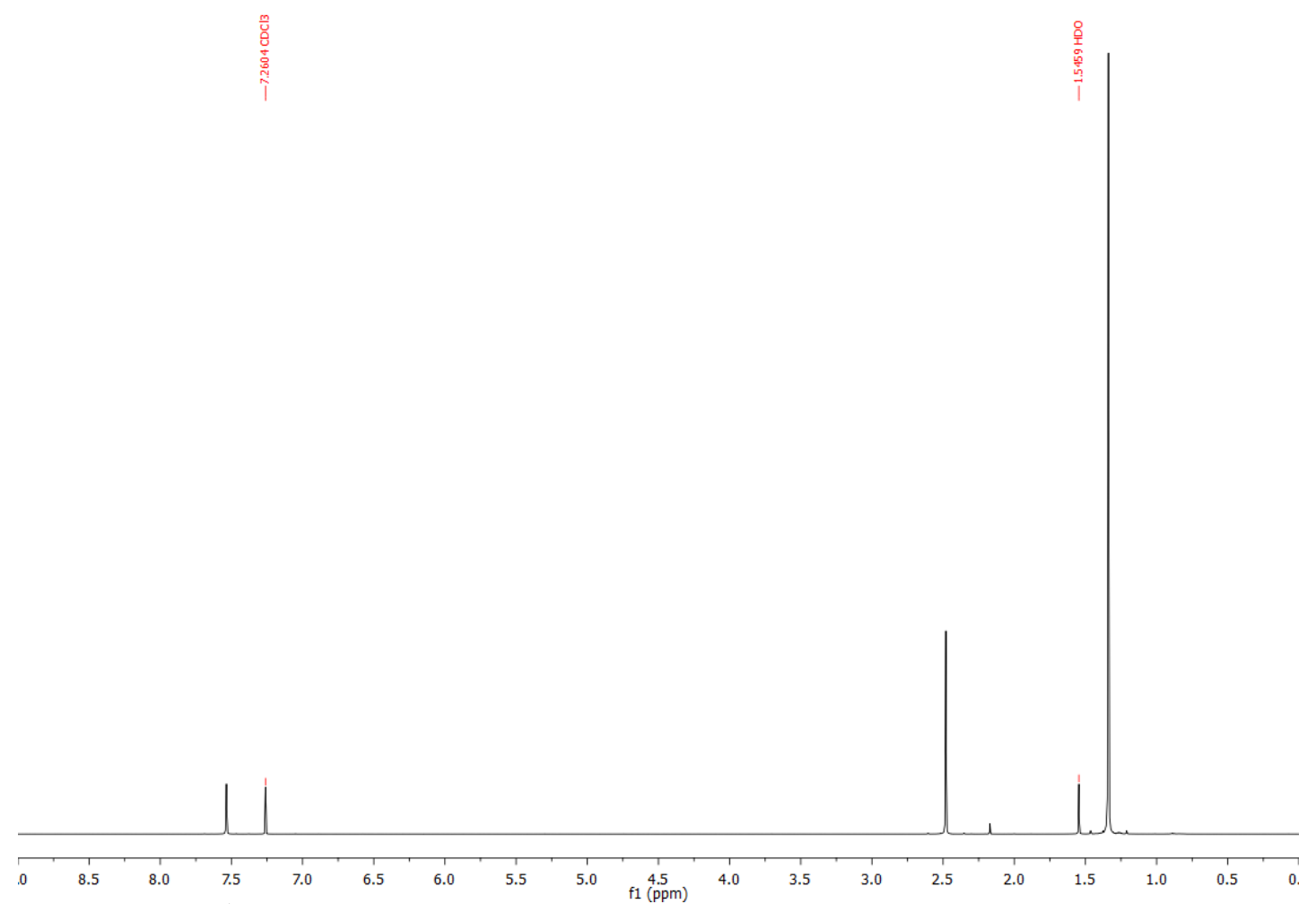

Figure S26: ${ }^{1} \mathrm{H}$ NMR Spectrum (500 MHZ, $\mathrm{CDCl}_{3}$ ) of 2,5-dimethyl-1,4-benzene diboronic acid bis(pinacol) ester. 


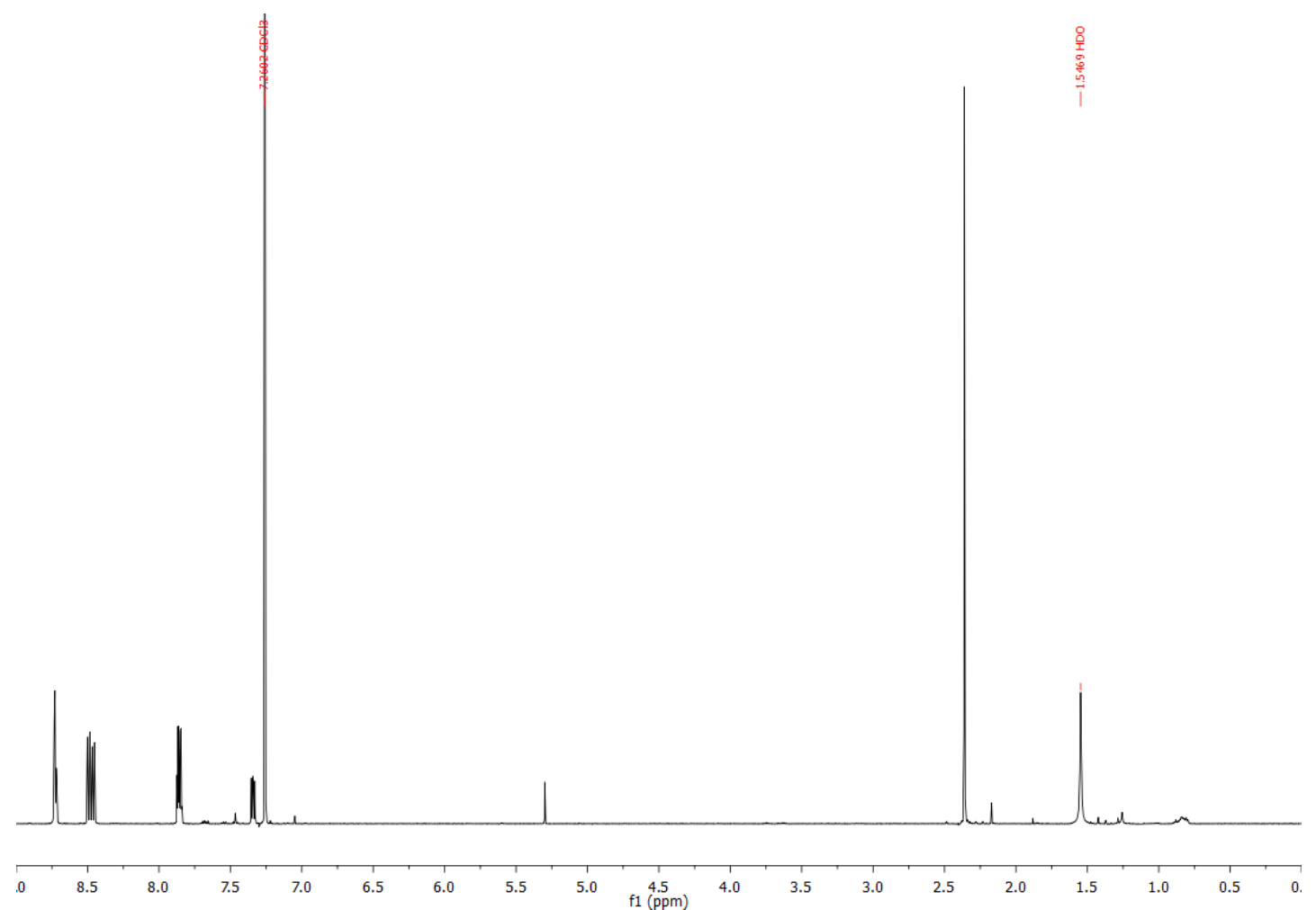

Figure S27: ${ }^{1} \mathrm{H}$ NMR Spectrum (500 $\left.\mathrm{MHZ}, \mathrm{CDCl}_{3}\right)$ of 1,4-bis(5-bipyridin-2,2'-yl)-2,5dimethyl-1,4-benzene ( $\left.\mathbf{L}^{\mathbf{d}}\right)$.

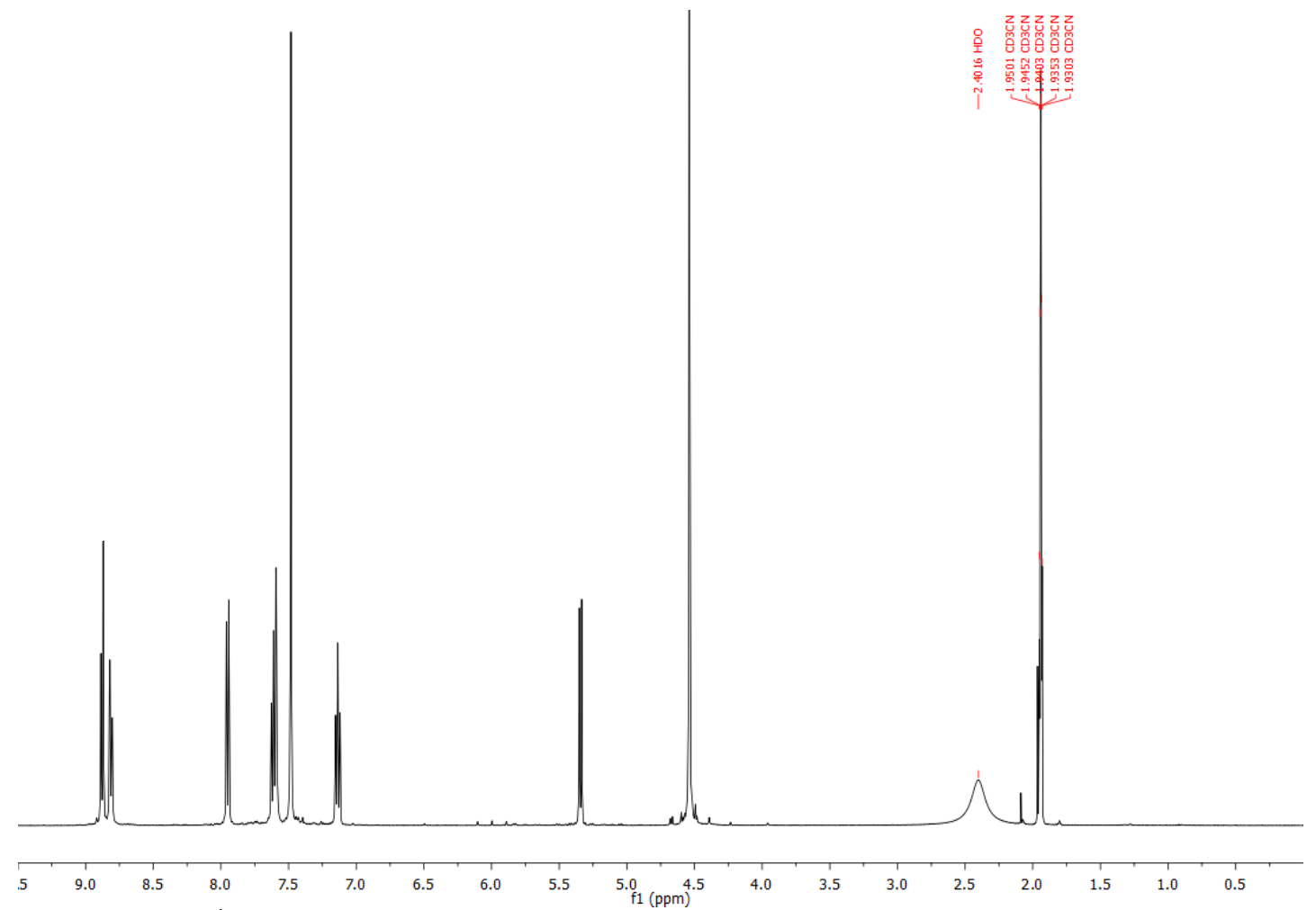

Figure S28: ${ }^{1} \mathrm{H}$ NMR Spectrum (500 $\left.\mathrm{MHZ}, \mathrm{CD}_{3} \mathrm{CN}\right)$ of $\mathbf{1 b} \cdot 12 \mathrm{PF}_{6}$. 


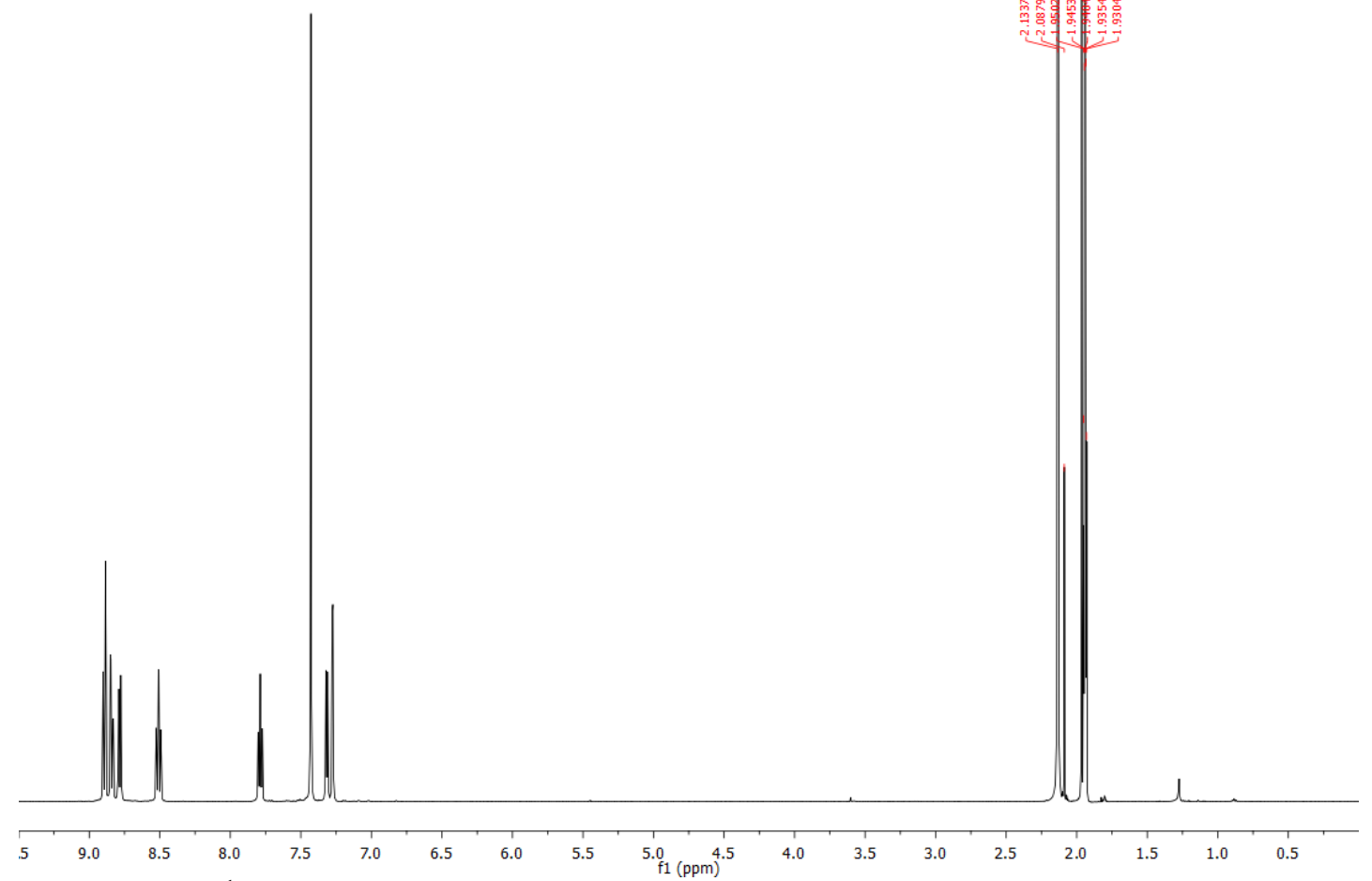

Figure S29: ${ }^{1} \mathrm{H}$ NMR Spectrum (500 MHZ, $\left.\mathrm{CD}_{3} \mathrm{CN}\right)$ of $1 \mathrm{c} \cdot 12 \mathrm{PF}_{6}$.

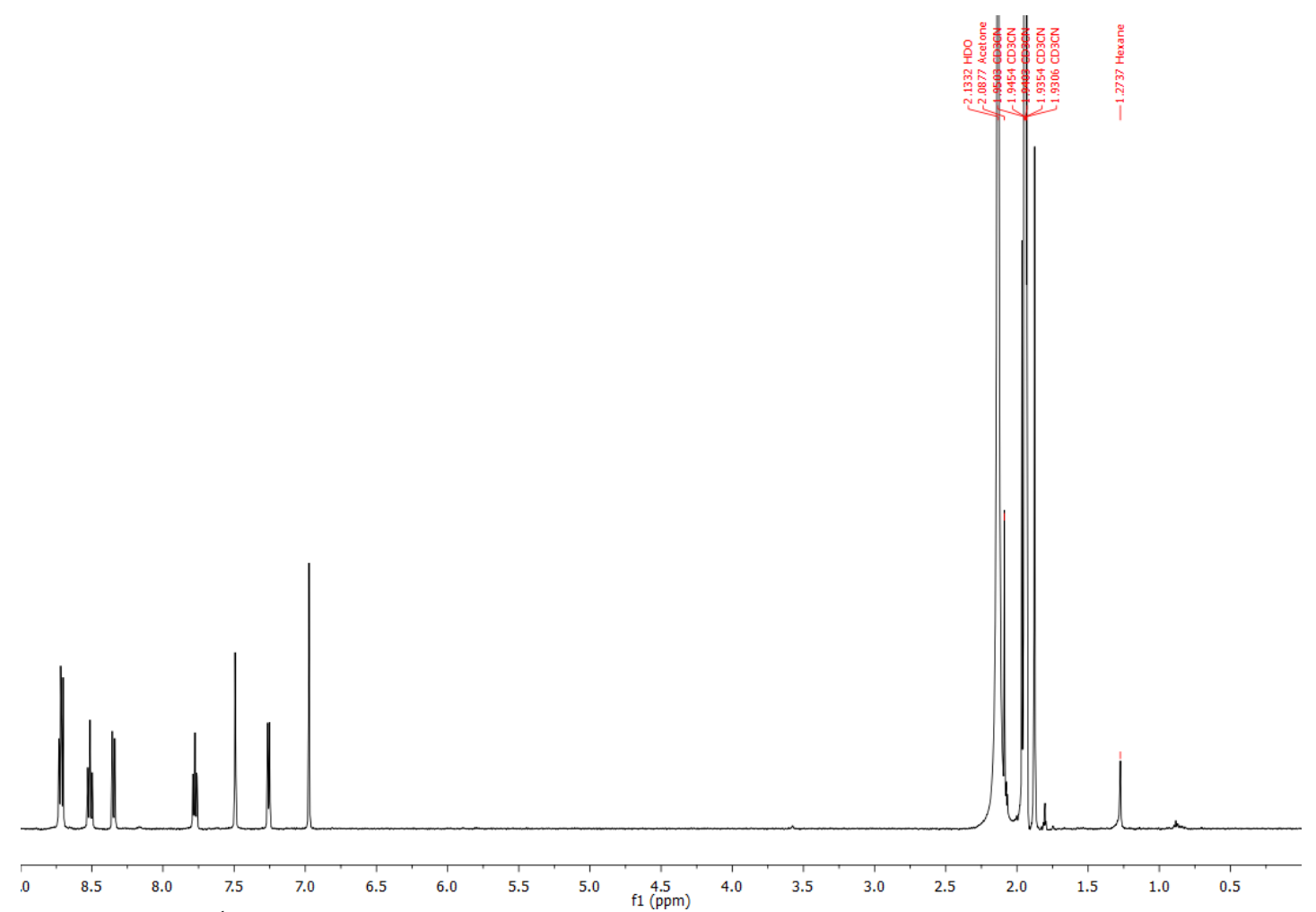

Figure S30: ${ }^{1} \mathrm{H}$ NMR Spectrum (500 MHZ, $\left.\mathrm{CD}_{3} \mathrm{CN}\right)$ of $\mathbf{1 d} \cdot 12 \mathrm{PF}_{6}$. 


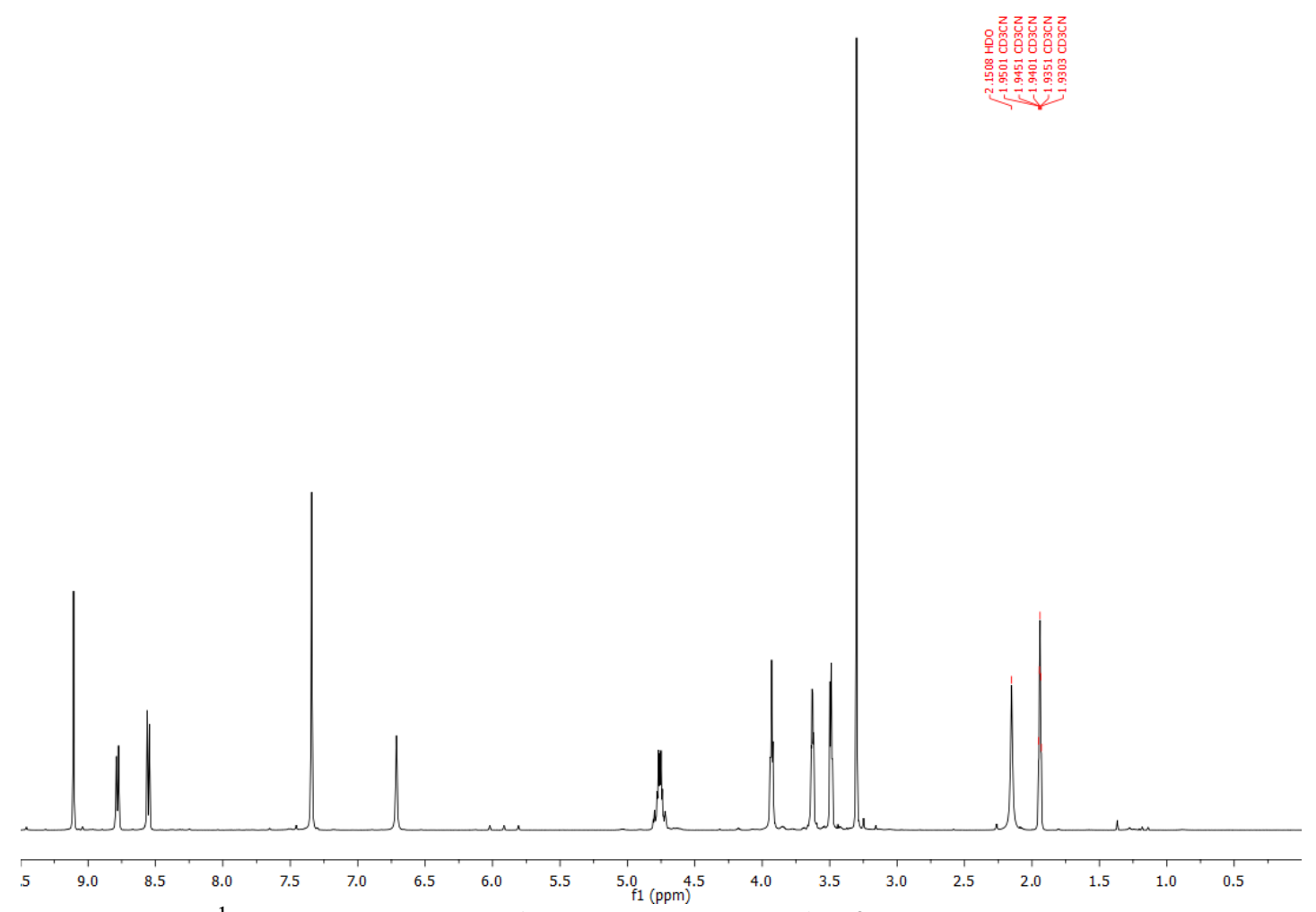

Figure S31: ${ }^{1} \mathrm{H}$ NMR Spectrum (500 MHZ, $\left.\mathrm{CD}_{3} \mathrm{CN}\right)$ of $\mathbf{2 a} \cdot 6 \mathrm{PF}_{6}$.

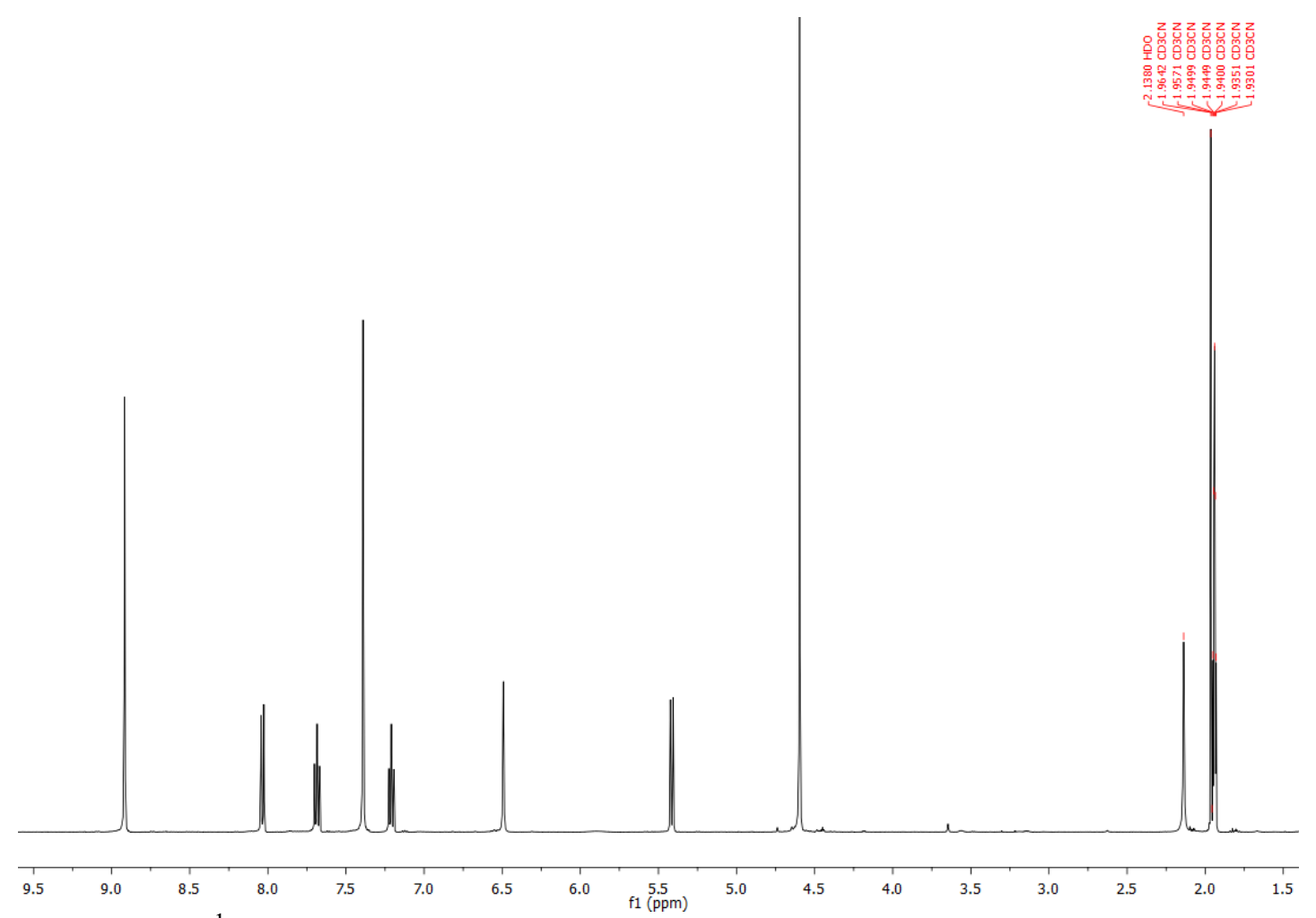

Figure S32: ${ }^{1} \mathrm{H}$ NMR Spectrum (500 MHZ, $\left.\mathrm{CD}_{3} \mathrm{CN}\right)$ of $\mathbf{2 b} \cdot 6 \mathrm{PF}_{6}$. 


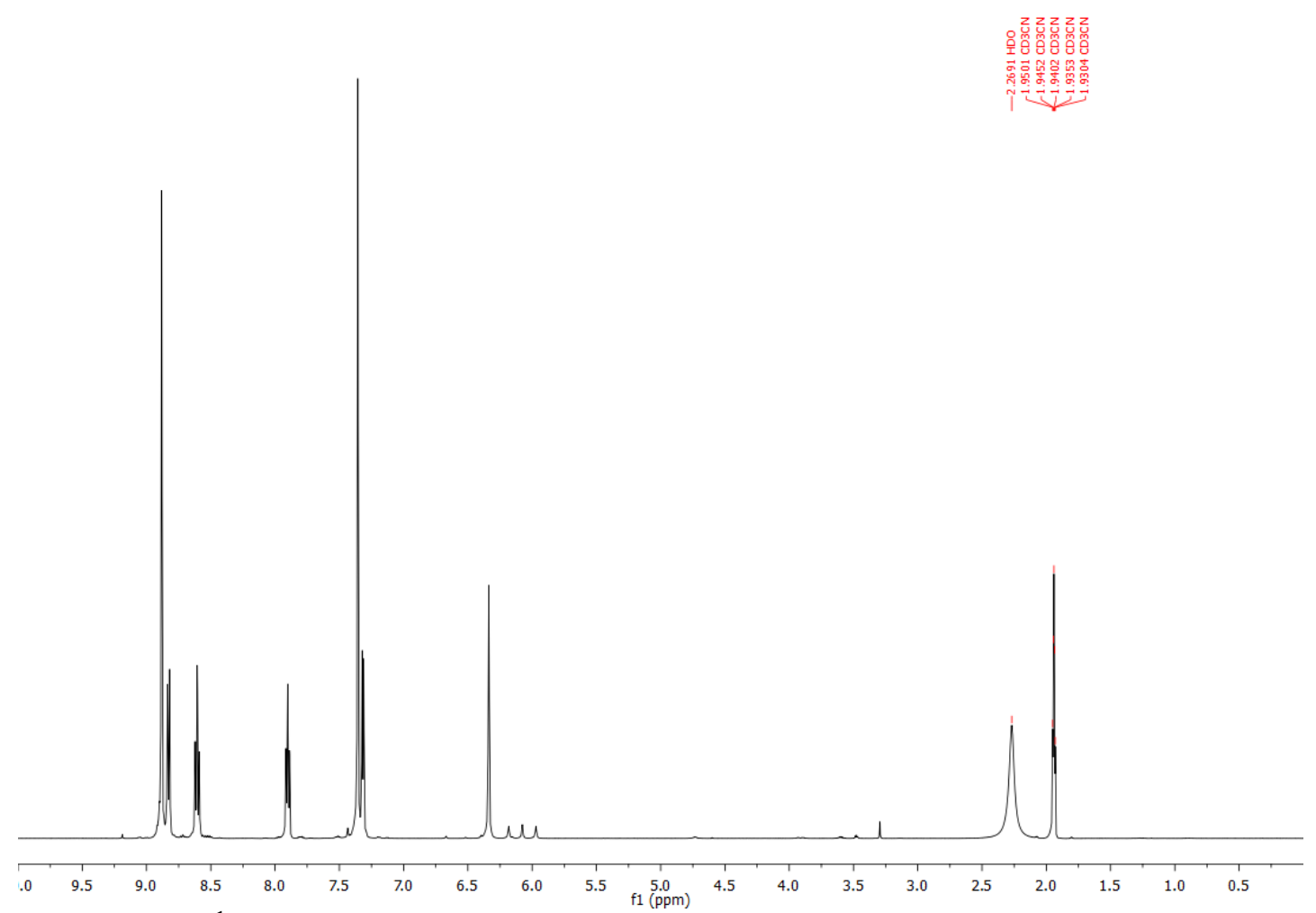

Figure S33: ${ }^{1} \mathrm{H}$ NMR Spectrum $\left(500 \mathrm{MHZ}, \mathrm{CD}_{3} \mathrm{CN}\right)$ of $2 \mathbf{c} \cdot 6 \mathrm{PF}_{6}$.

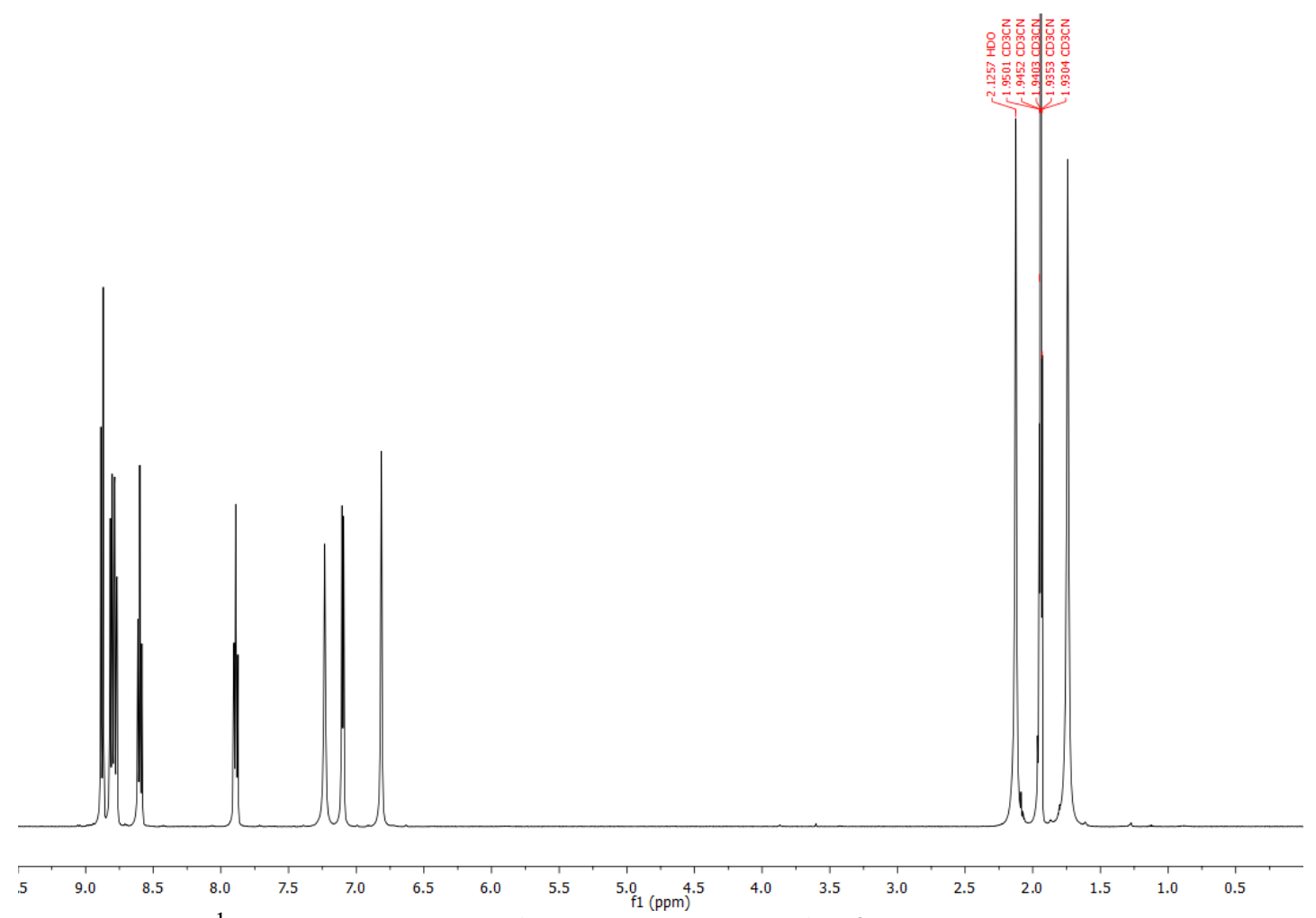

Figure S34: ${ }^{1} \mathrm{H}$ NMR Spectrum (500 MHZ, $\left.\mathrm{CD}_{3} \mathrm{CN}\right)$ of $\mathbf{2 d} \cdot 6 \mathrm{PF}_{6}$. 


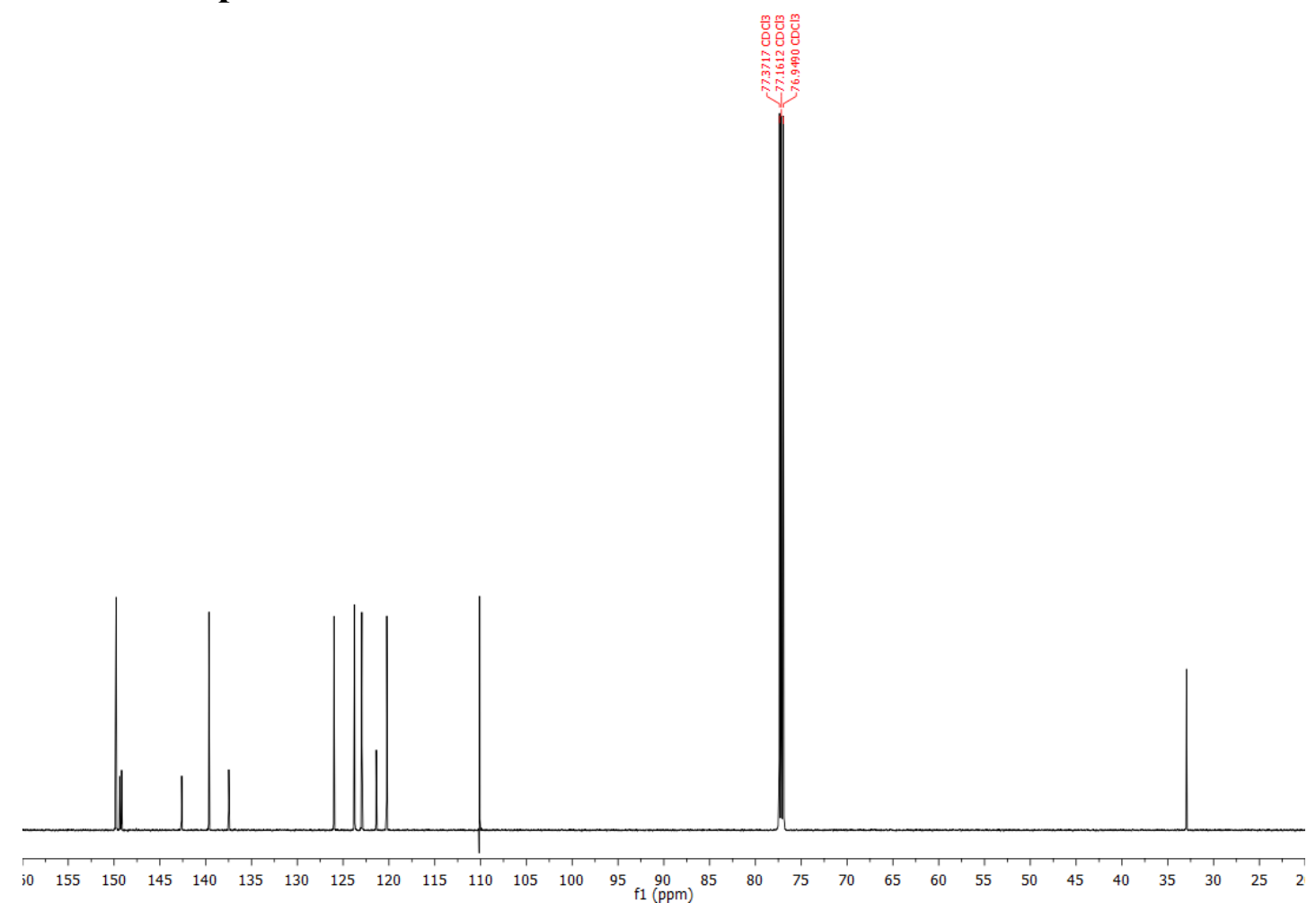

Figure S35: ${ }^{13} \mathrm{C}$ NMR Spectrum (151 $\left.\mathrm{MHZ}, \mathrm{CDCl}_{3}\right)$ of 2-(5-bromopyridin-2-yl)-1-methyl1,3-benzimidazole.

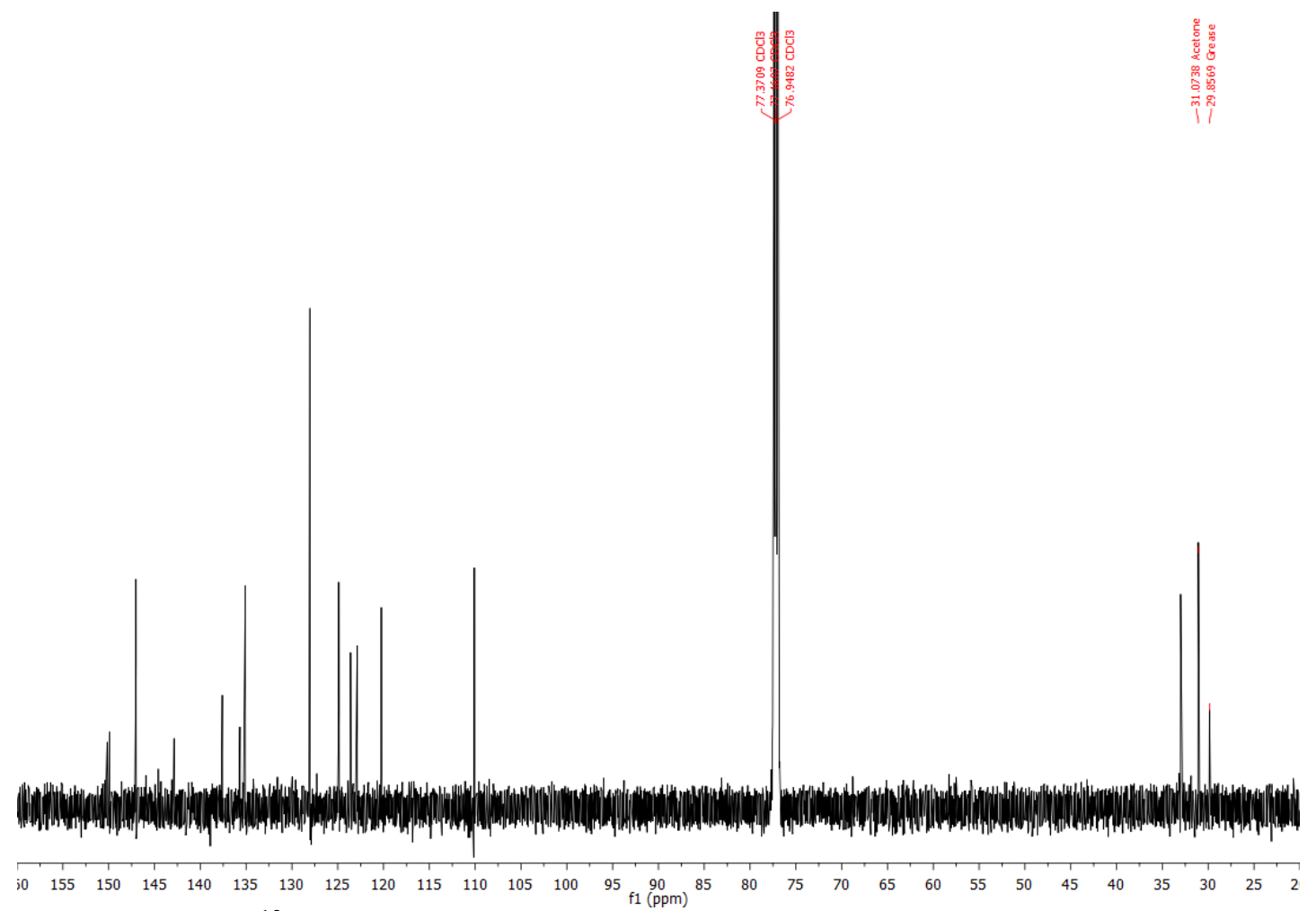

Figure S36: ${ }^{13} \mathrm{C}$ NMR Spectrum (151 $\left.\mathrm{MHZ}, \mathrm{CDCl}_{3}\right)$ of 1,4-bis(6-(1-methyl-1,3benzimidazolyl)pyridin-3-yl)benzene $\left(\mathbf{L}^{\mathbf{b}}\right)$. 


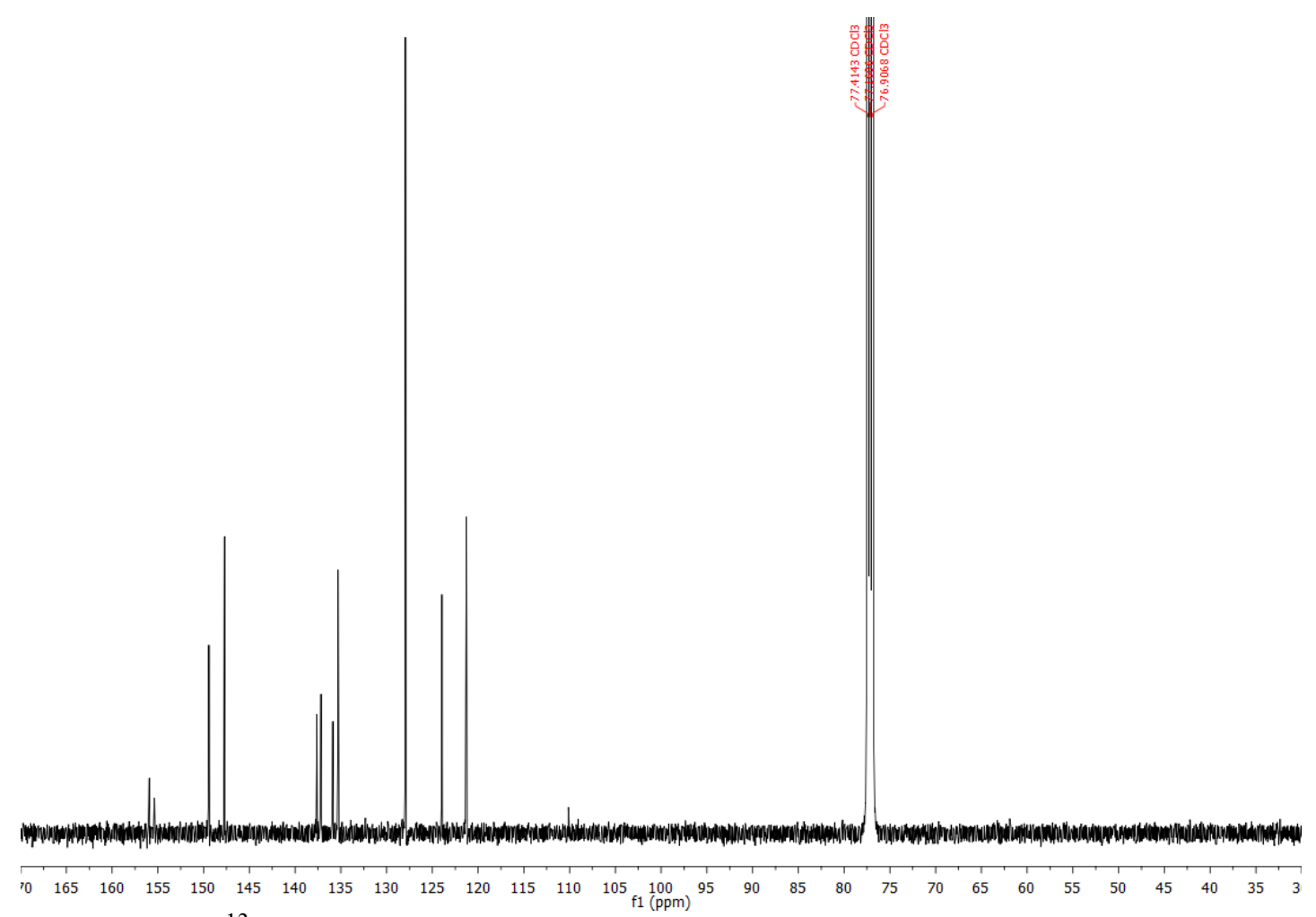

Figure S37: ${ }^{13} \mathrm{C}$ NMR Spectrum (126 $\mathrm{MHZ}, \mathrm{CDCl}_{3}$ ) of 1,4-bis(5-bipyridin-2,2'-yl)benzene $\left(\mathbf{L}^{\mathbf{c}}\right)$.

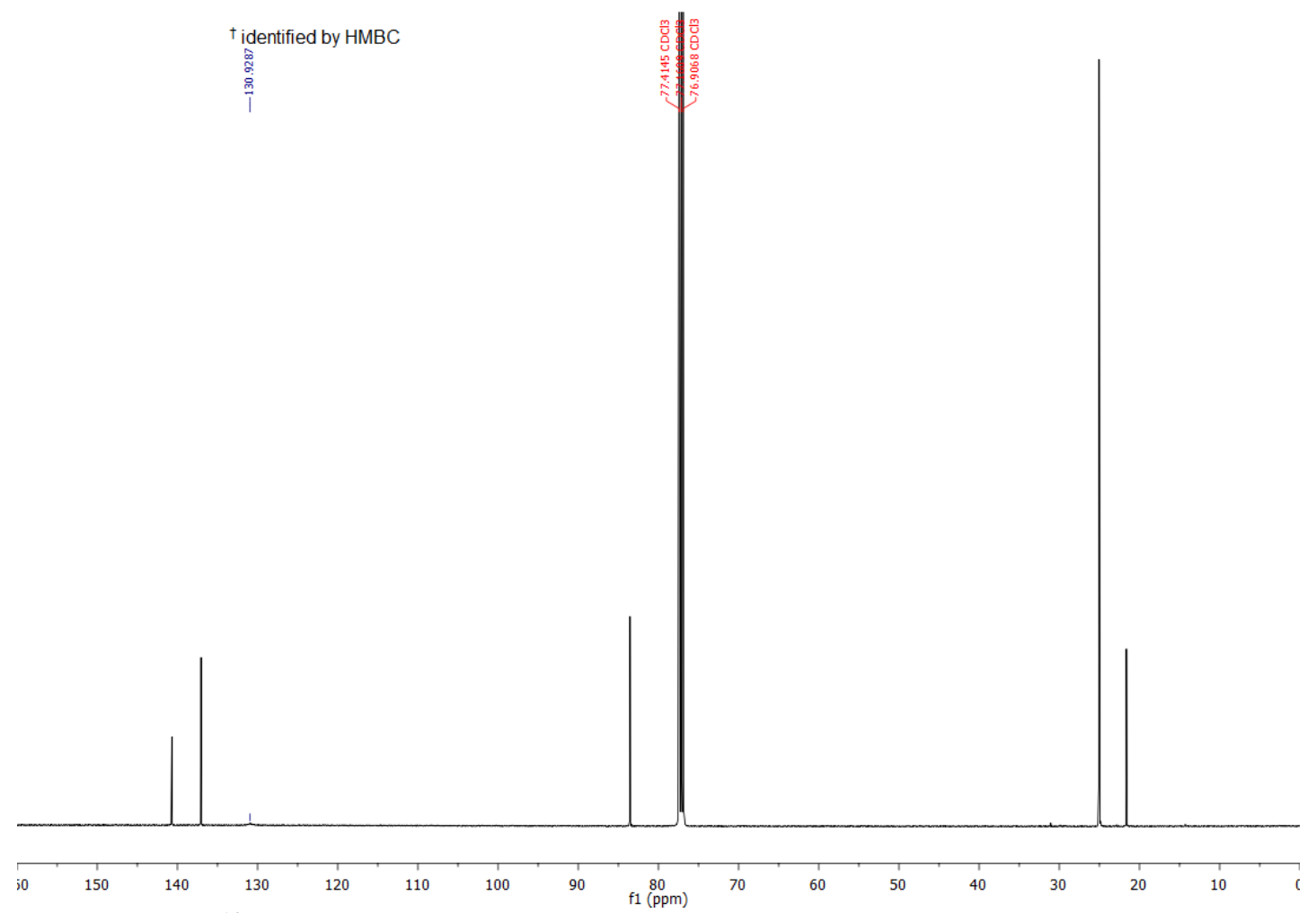

Figure S38: ${ }^{13} \mathrm{C}$ NMR Spectrum (126 $\left.\mathrm{MHZ}, \mathrm{CDCl}_{3}\right)$ of 2,5-dimethyl-1,4-benzene diboronic acid bis(pinacol) ester. 


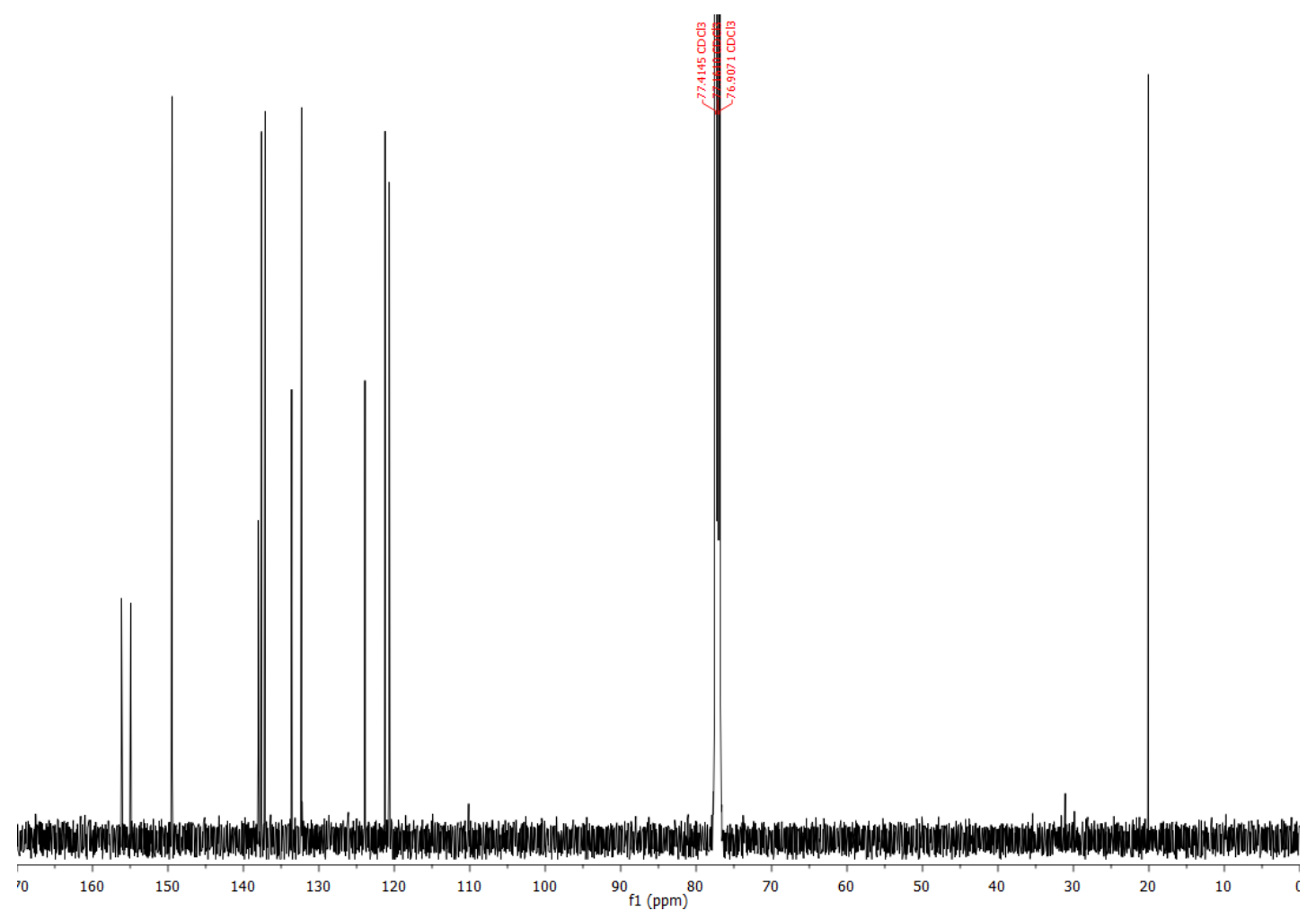

Figure S39: ${ }^{13} \mathrm{C}$ NMR Spectrum (126 $\left.\mathrm{MHZ}, \mathrm{CDCl}_{3}\right)$ of 1,4-bis(5-bipyridin-2,2'-yl)-2,5dimethyl-1,4-benzene $\left(\mathbf{L}^{\mathbf{d}}\right)$.

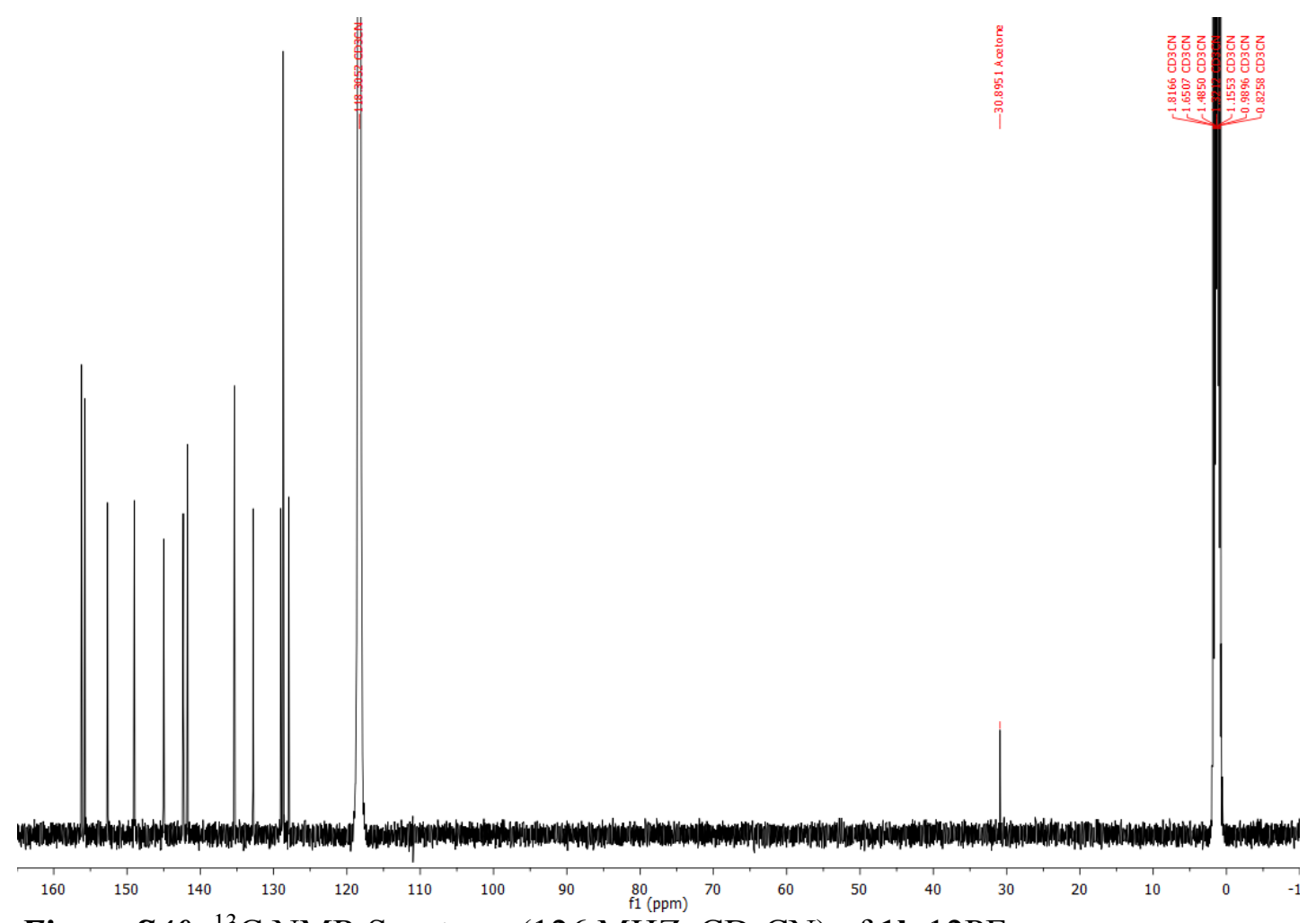

Figure S40: ${ }^{13} \mathrm{C}$ NMR Spectrum $\left(126 \mathrm{MHZ}, \mathrm{CD}_{3} \mathrm{CN}\right)$ of $\mathbf{1 b} \cdot 12 \mathrm{PF}_{6}$. 


$$
\text { I }
$$




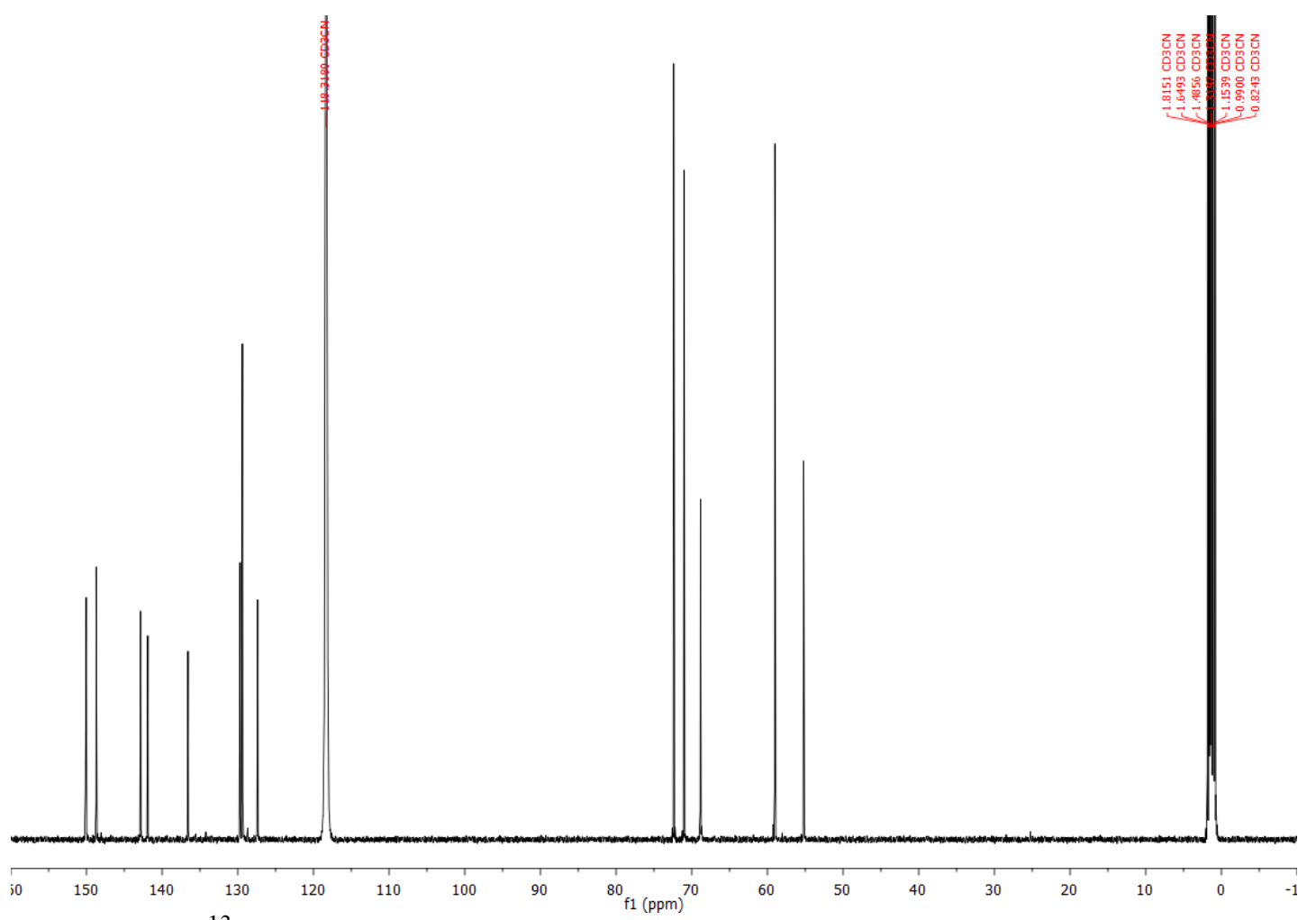

Figure S43: ${ }^{13} \mathrm{C}$ NMR Spectrum $\left(126 \mathrm{MHZ}, \mathrm{CD}_{3} \mathrm{CN}\right)$ of $\mathbf{2 a} \cdot 6 \mathrm{PF}_{6}$.

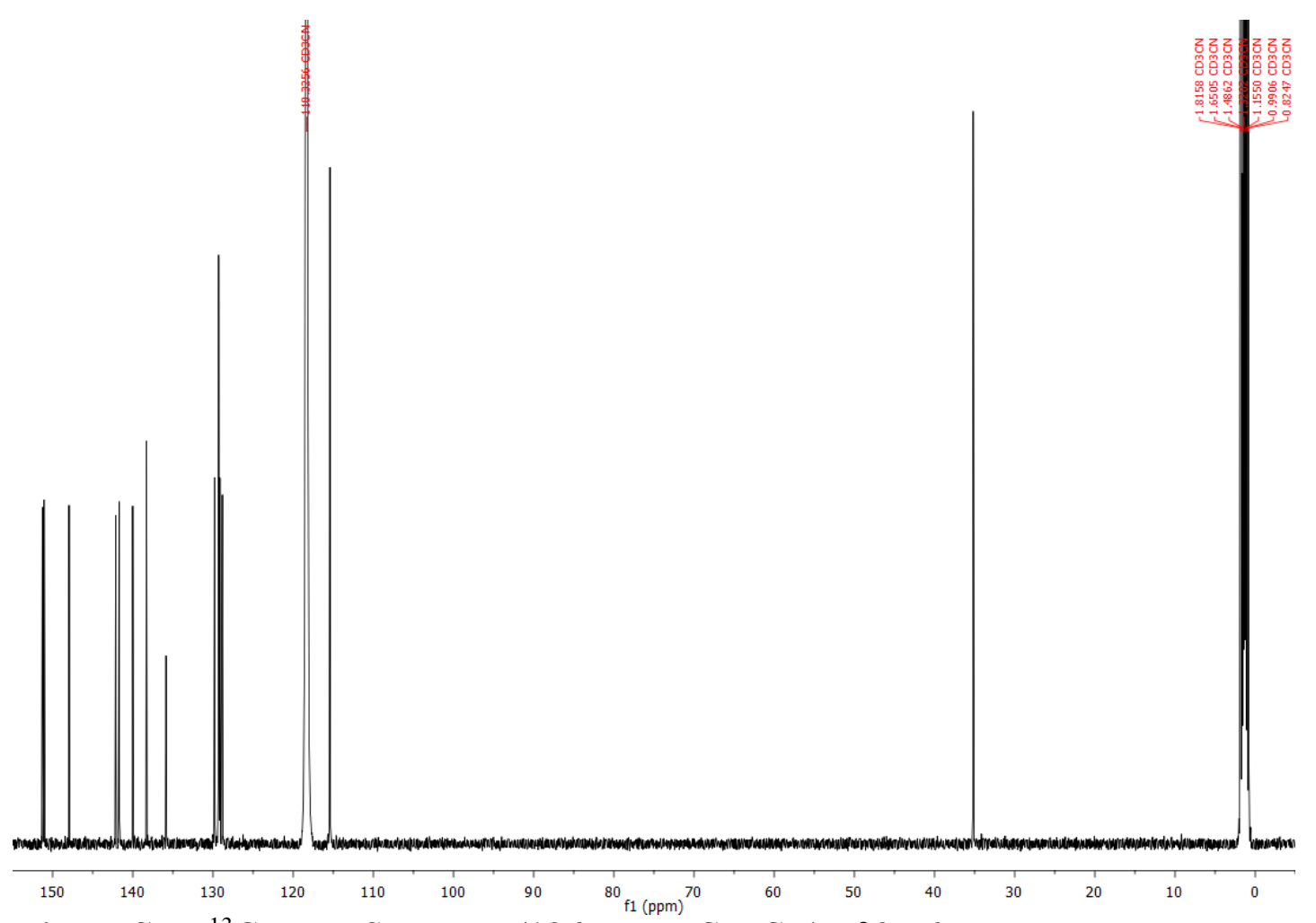

Figure S44: ${ }^{13} \mathrm{C}$ NMR Spectrum (126 MHZ, $\left.\mathrm{CD}_{3} \mathrm{CN}\right)$ of $\mathbf{2 b} \cdot 6 \mathrm{PF}_{6}$.

S40 


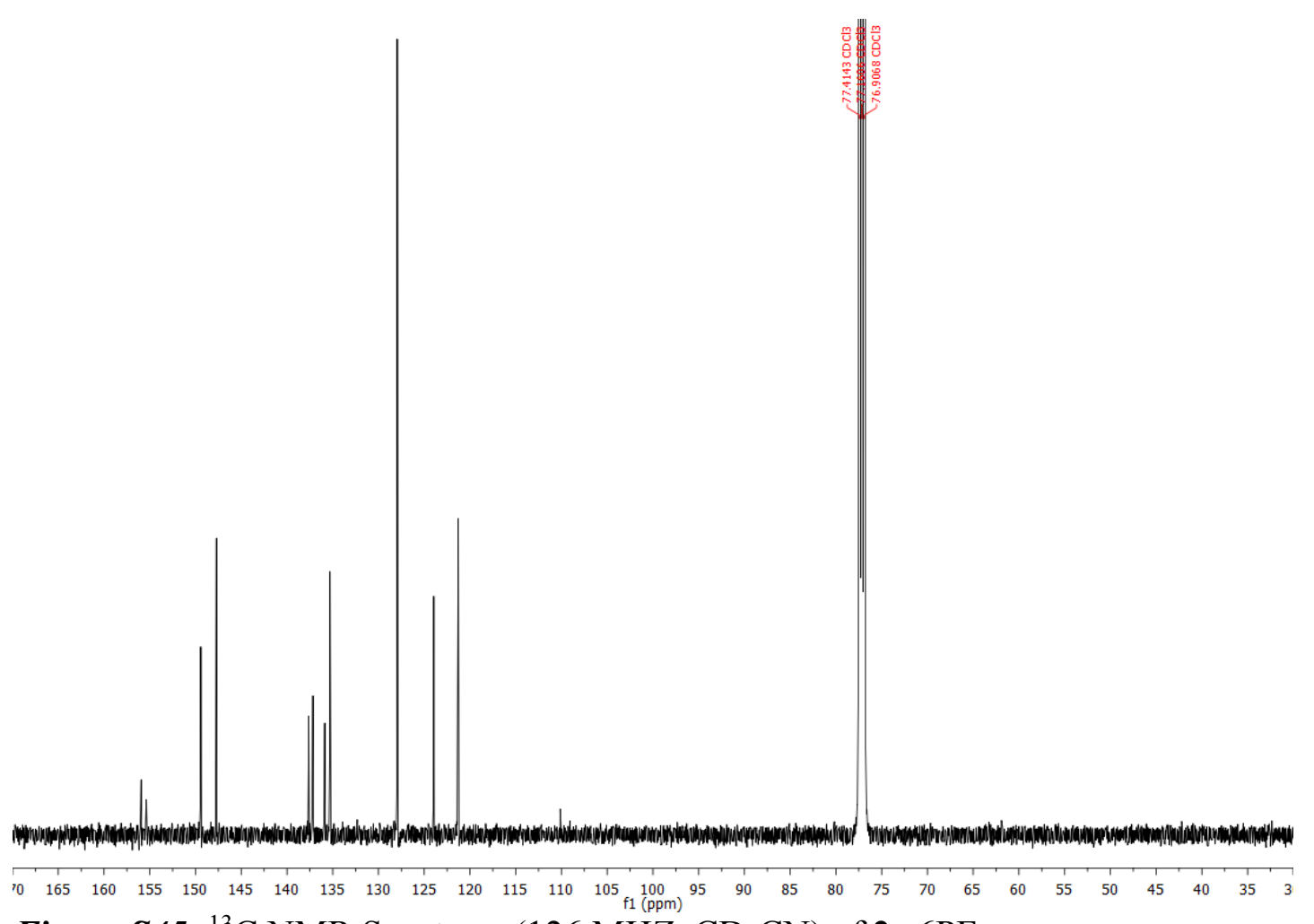

Figure S45: ${ }^{13} \mathrm{C}$ NMR Spectrum $\left(126 \mathrm{MHZ}, \mathrm{CD}_{3} \mathrm{CN}\right)$ of $2 \mathrm{c} \cdot 6 \mathrm{PF}_{6}$.

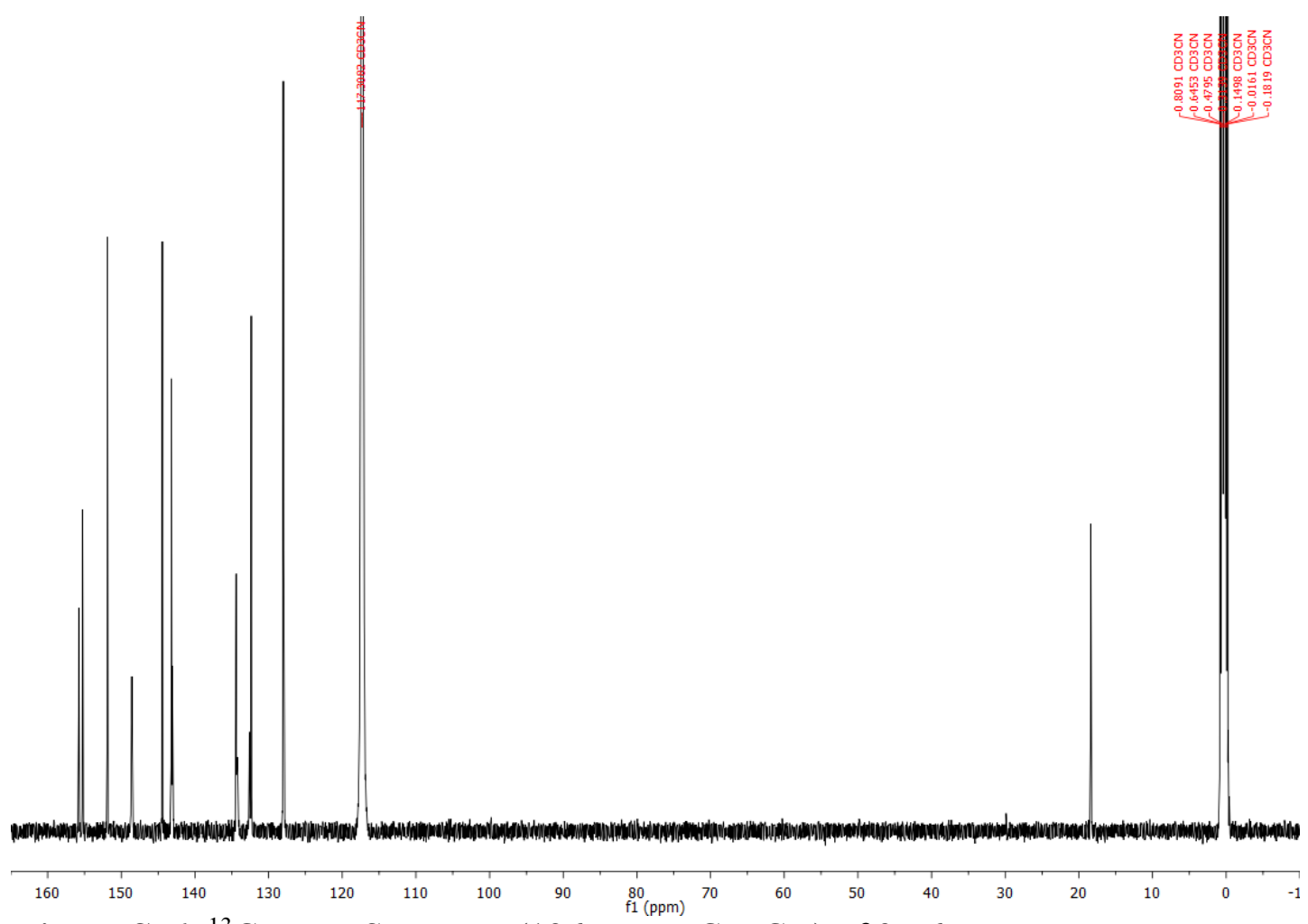

Figure S46: ${ }^{13} \mathrm{C}$ NMR Spectrum (126 MHZ, $\left.\mathrm{CD}_{3} \mathrm{CN}\right)$ of $\mathbf{2 d} \cdot 6 \mathrm{PF}_{6}$.

S41 


\section{${ }^{1}$ H DOSY Spectra}

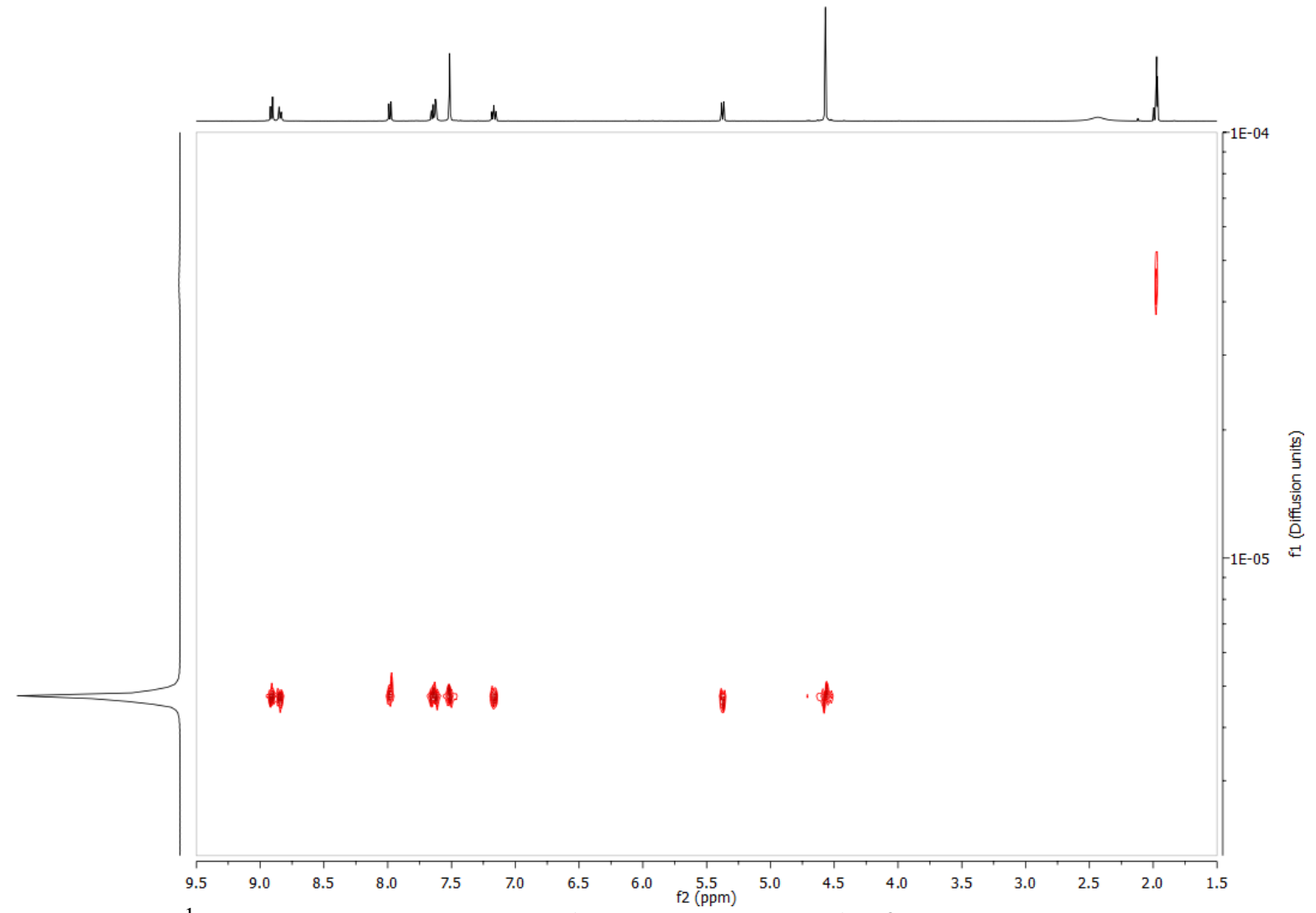

Figure S47: ${ }^{1} \mathrm{H}$ DOSY NMR Spectrum $\left(500 \mathrm{MHZ}, \mathrm{CD}_{3} \mathrm{CN}\right)$ of $\mathbf{1 b} \cdot 12 \mathrm{PF}_{6}$.

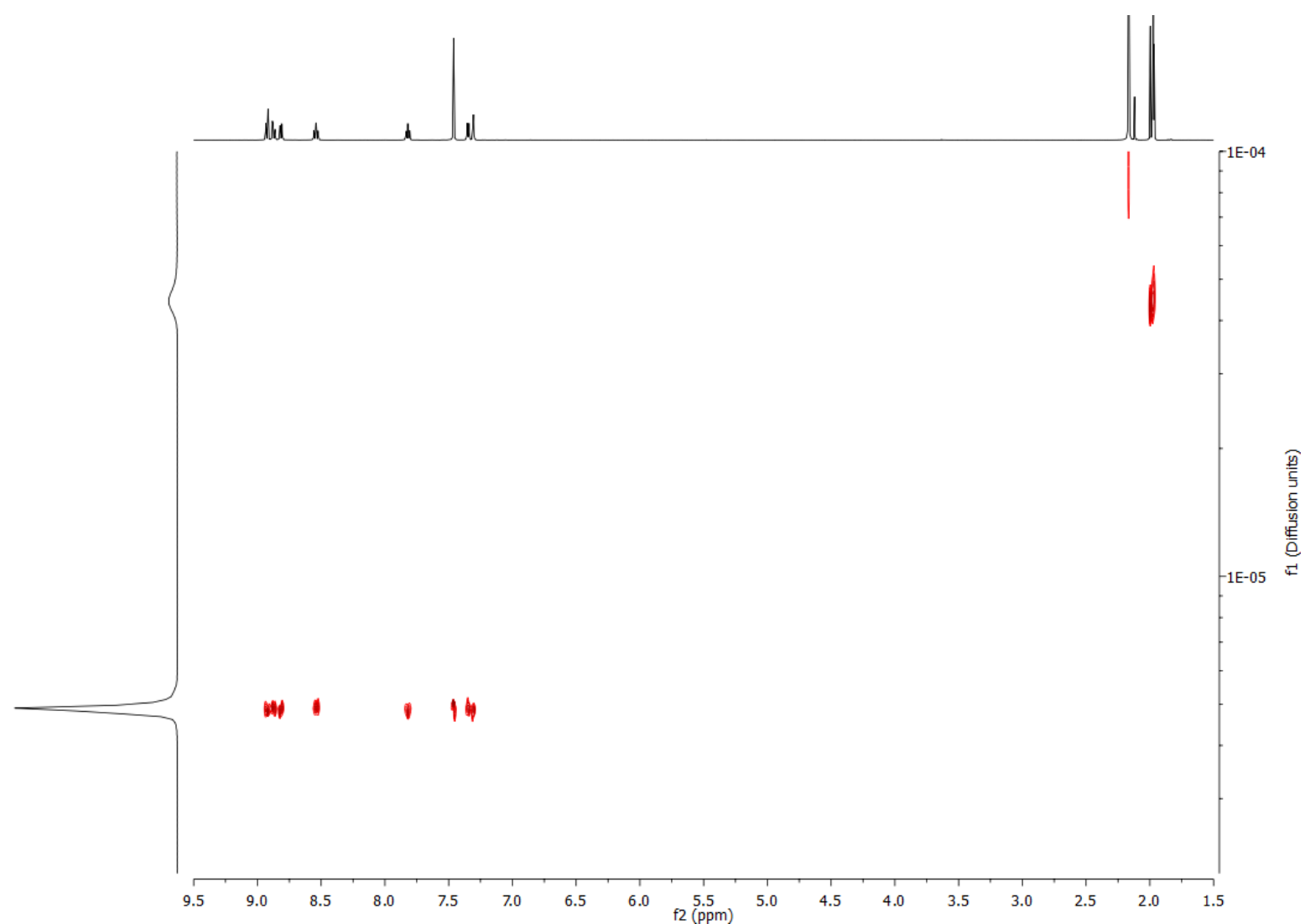

Figure S48: ${ }^{1} \mathrm{H}$ DOSY NMR Spectrum ( $\left.500 \mathrm{MHZ}, \mathrm{CD}_{3} \mathrm{CN}\right)$ of $1 \mathrm{c} \cdot 12 \mathrm{PF}_{6}$. 


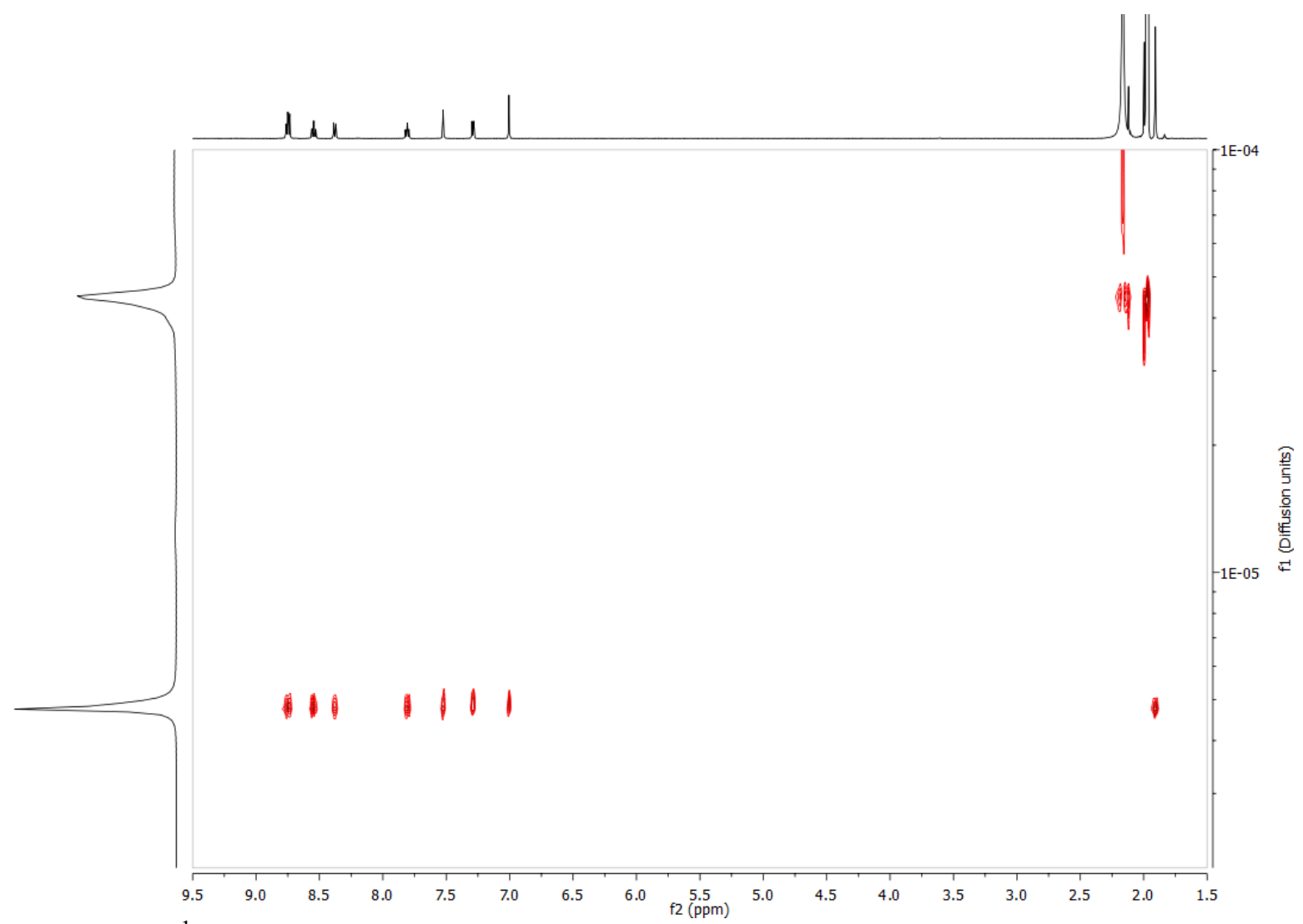

Figure S49: ${ }^{1} \mathrm{H}$ DOSY NMR Spectrum $\left(500 \mathrm{MHZ}, \mathrm{CD}_{3} \mathrm{CN}\right)$ of $\mathbf{1 d} \cdot 12 \mathrm{PF}_{6}$.

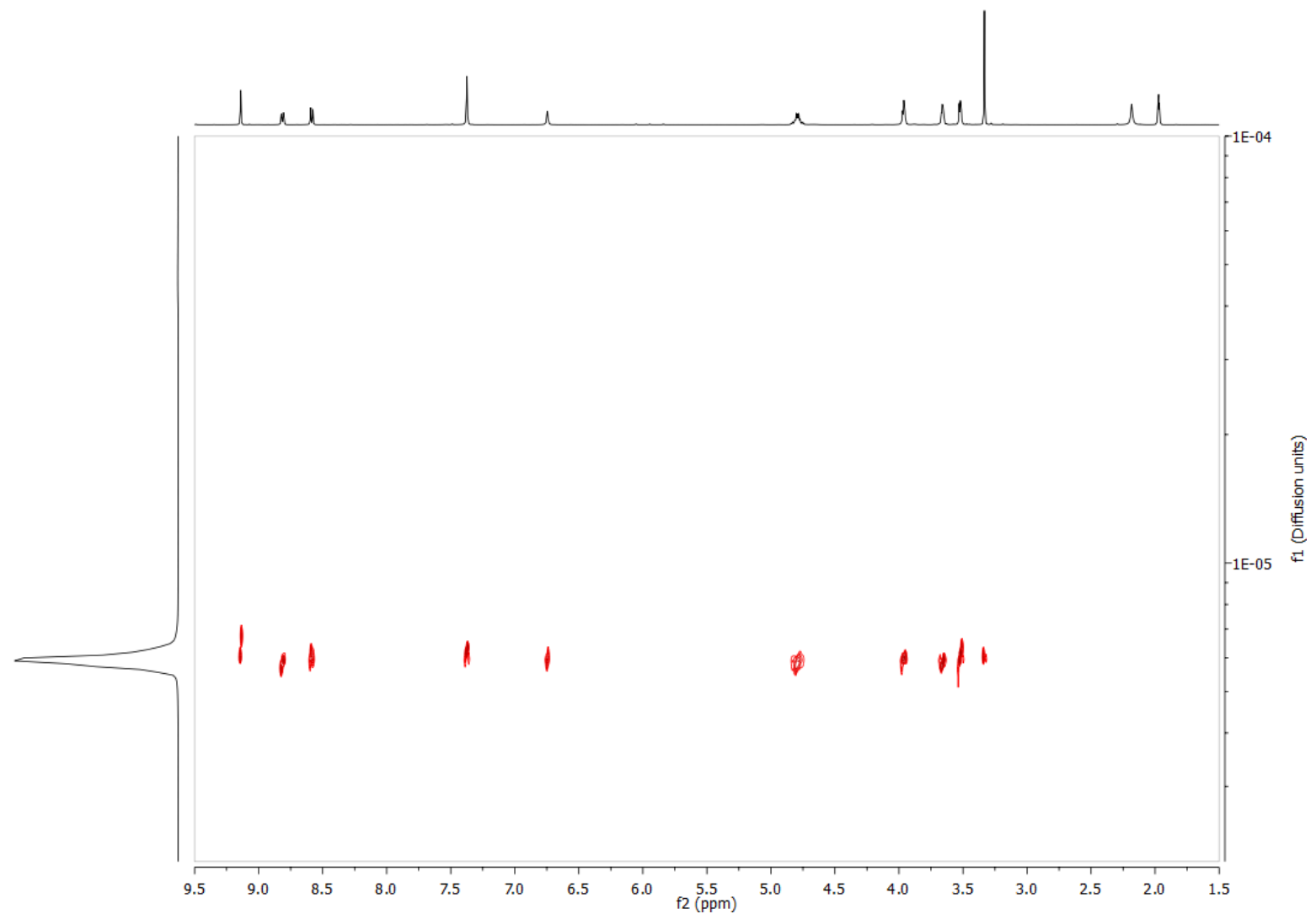

Figure S50: ${ }^{1} \mathrm{H}$ DOSY NMR Spectrum (500 MHZ, $\left.\mathrm{CD}_{3} \mathrm{CN}\right)$ of $\mathbf{2 a} \cdot 6 \mathrm{PF}_{6}$. 


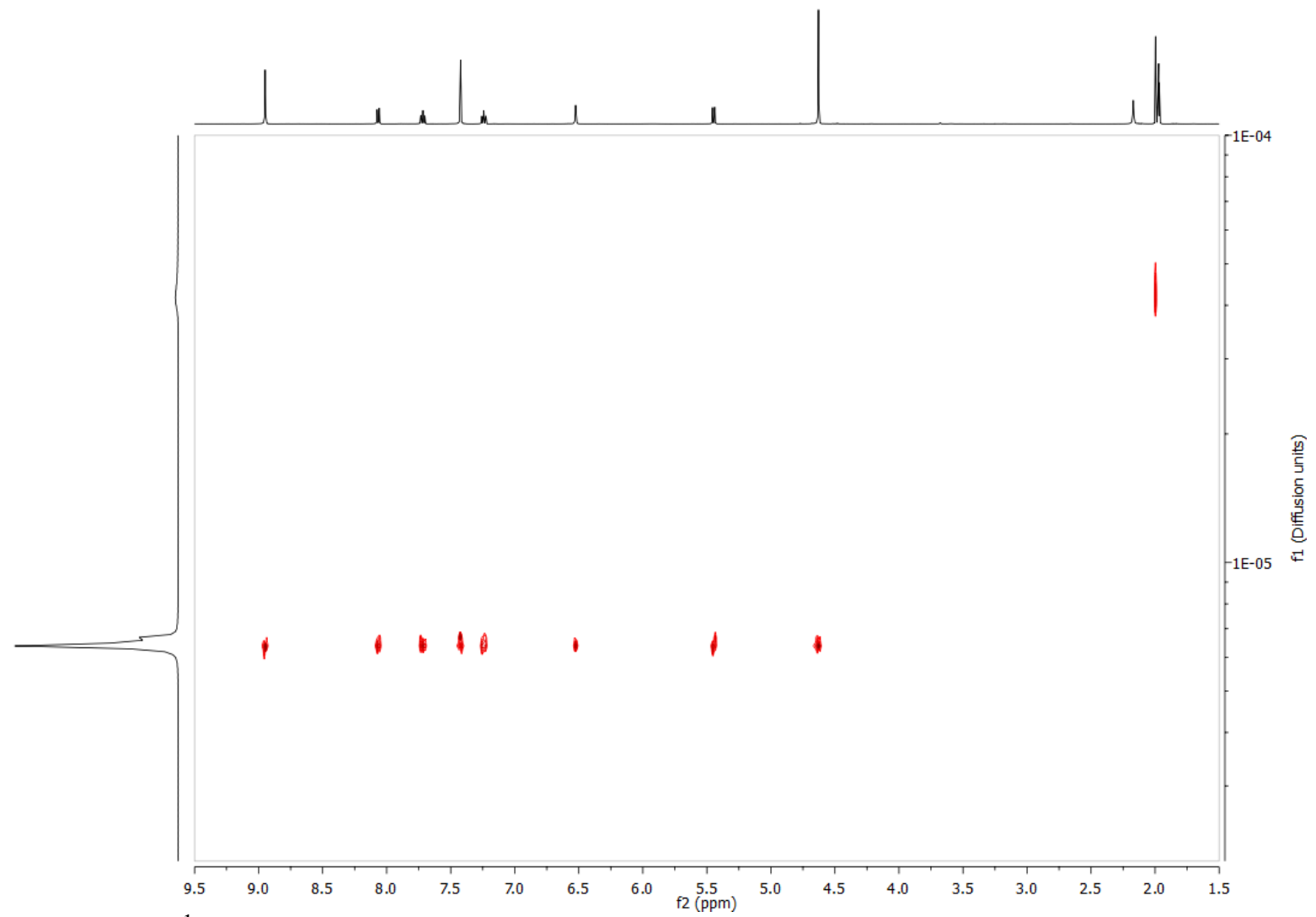

Figure S51: ${ }^{1} \mathrm{H}$ DOSY NMR Spectrum (500 MHZ, $\left.\mathrm{CD}_{3} \mathrm{CN}\right)$ of $\mathbf{2} \mathbf{b} \cdot 6 \mathrm{PF}_{6}$.

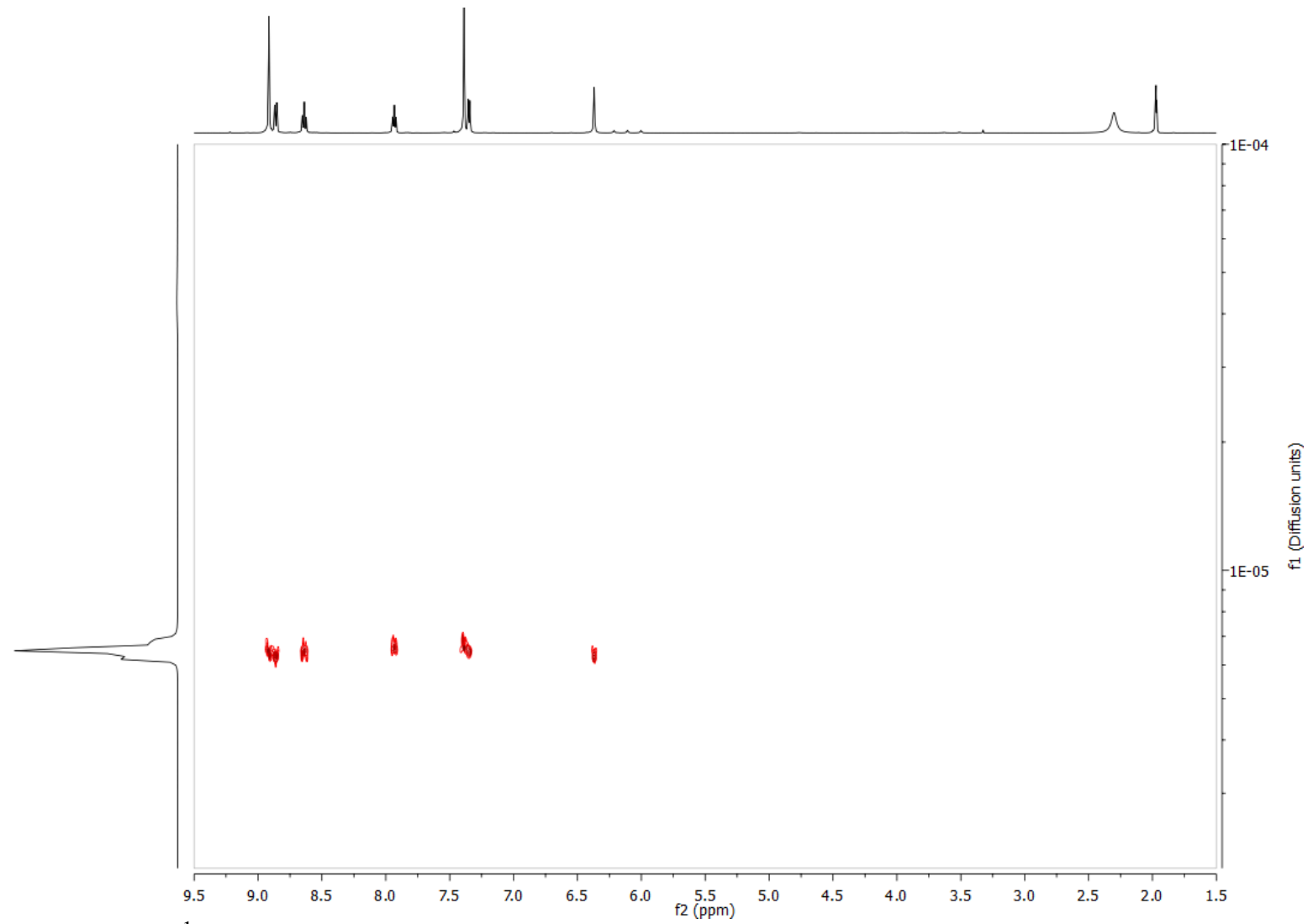

Figure S52: ${ }^{1} \mathrm{H}$ DOSY NMR Spectrum (500 MHZ, $\mathrm{CD}_{3} \mathrm{CN}$ ) of $2 \mathrm{c} \cdot 6 \mathrm{PF}_{6}$. 


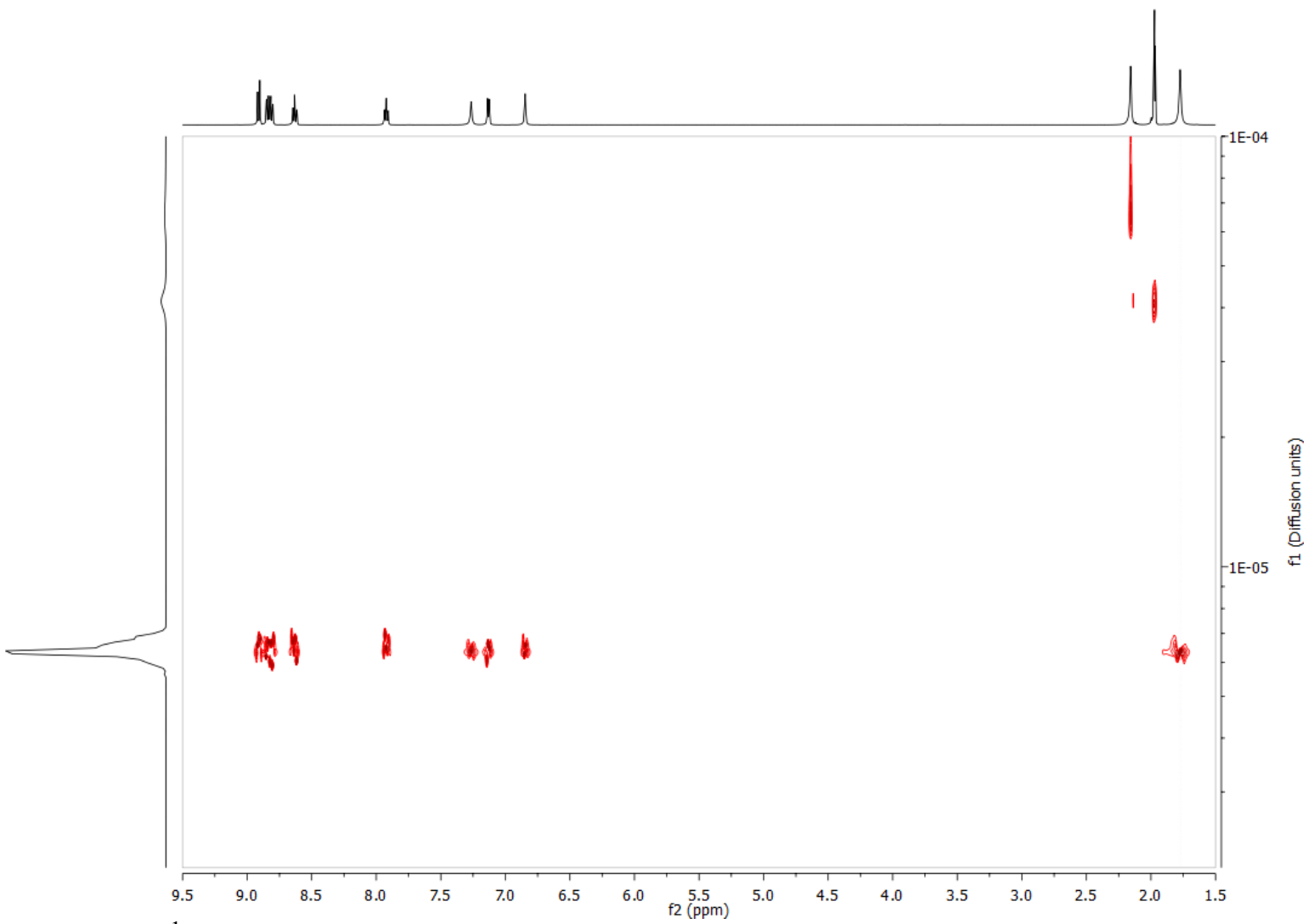

Figure S53: ${ }^{1} \mathrm{H}$ DOSY NMR Spectrum $\left(500 \mathrm{MHZ}, \mathrm{CD}_{3} \mathrm{CN}\right)$ of $\mathbf{2 d} \cdot 6 \mathrm{PF}_{6}$. 


\section{Mass Spectrometry of all complexes}

1b $\cdot 12 \mathrm{PF}_{6}$
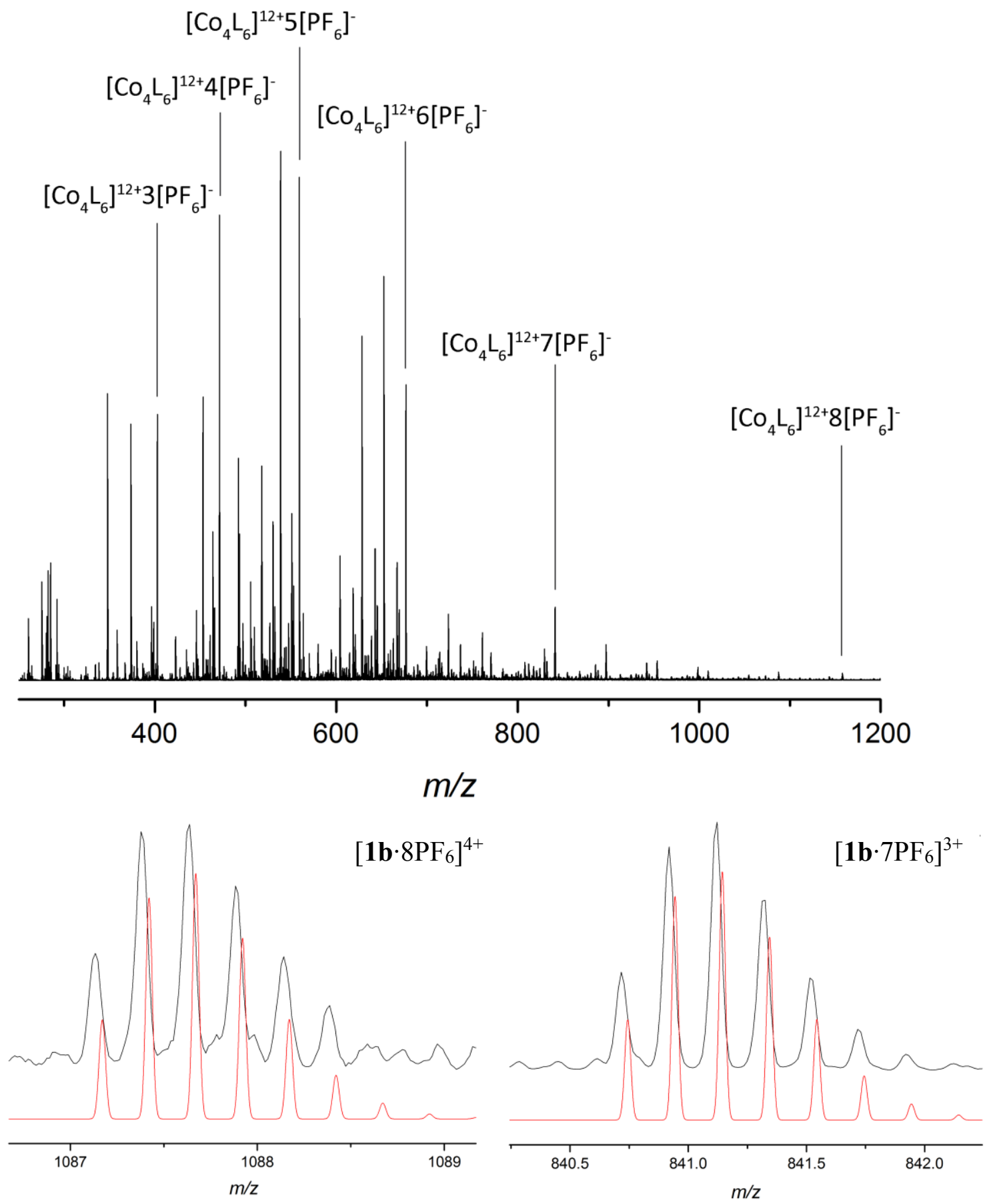

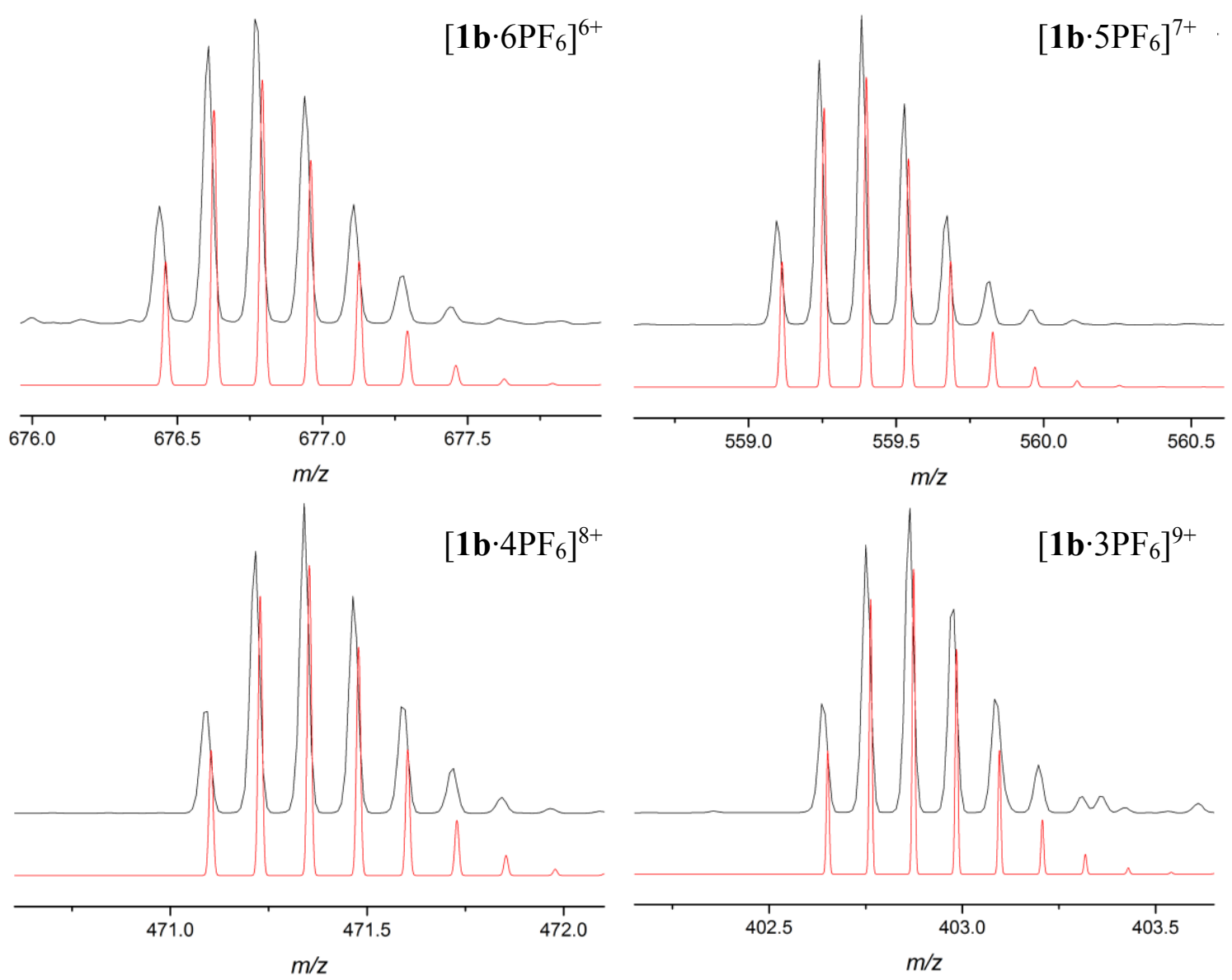

Figure S54: Mass Spectrum of $\mathbf{1 b} \cdot 12 \mathrm{PF}_{6}$. Full spectrum (top) is shown with expansions of the labelled peaks (bottom) to demonstrate isotopic distribution. Recorded data (black) is shown against modelled data for the tetrahedron (red). 
1c. $12 \mathrm{PF}_{6}$
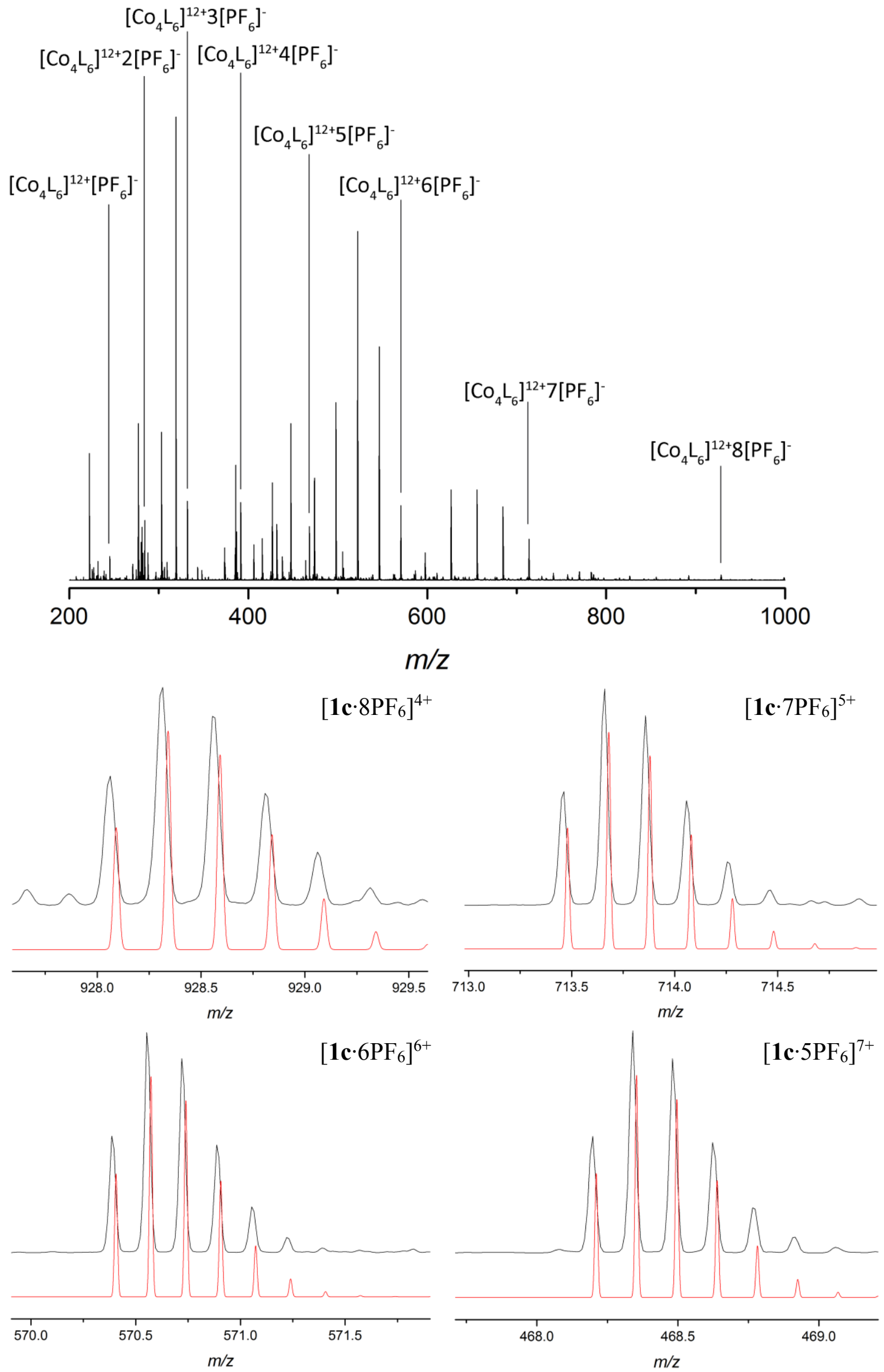

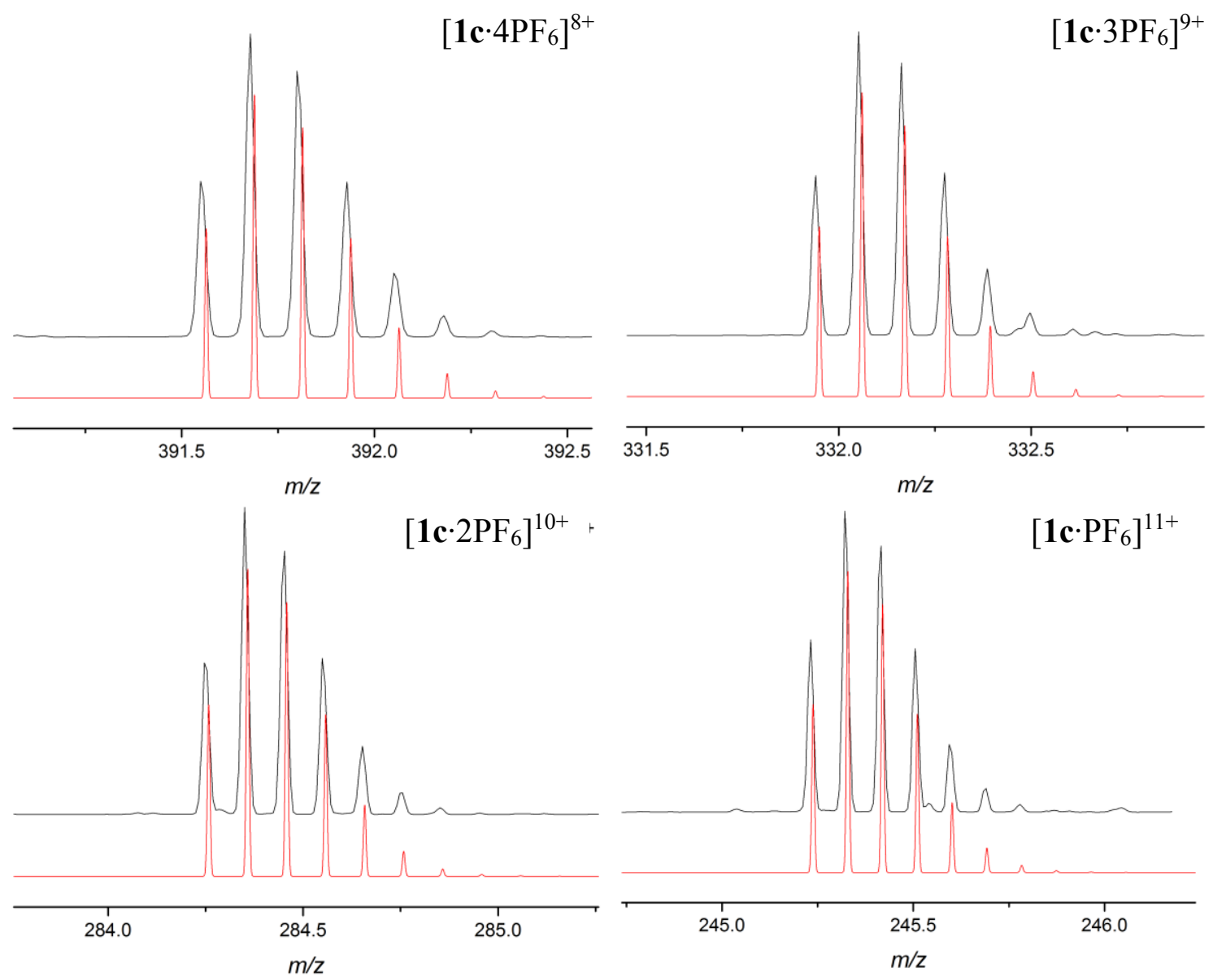

Figure S55: Mass Spectrum of 1c $12 \mathrm{PF}_{6}$. Full spectrum (top) is shown with expansions of the labelled peaks (bottom) to demonstrate isotopic distribution. Recorded data (black) is shown against modelled data for the tetrahedron (red). 
1d $\cdot 12 \mathrm{PF}_{6}$
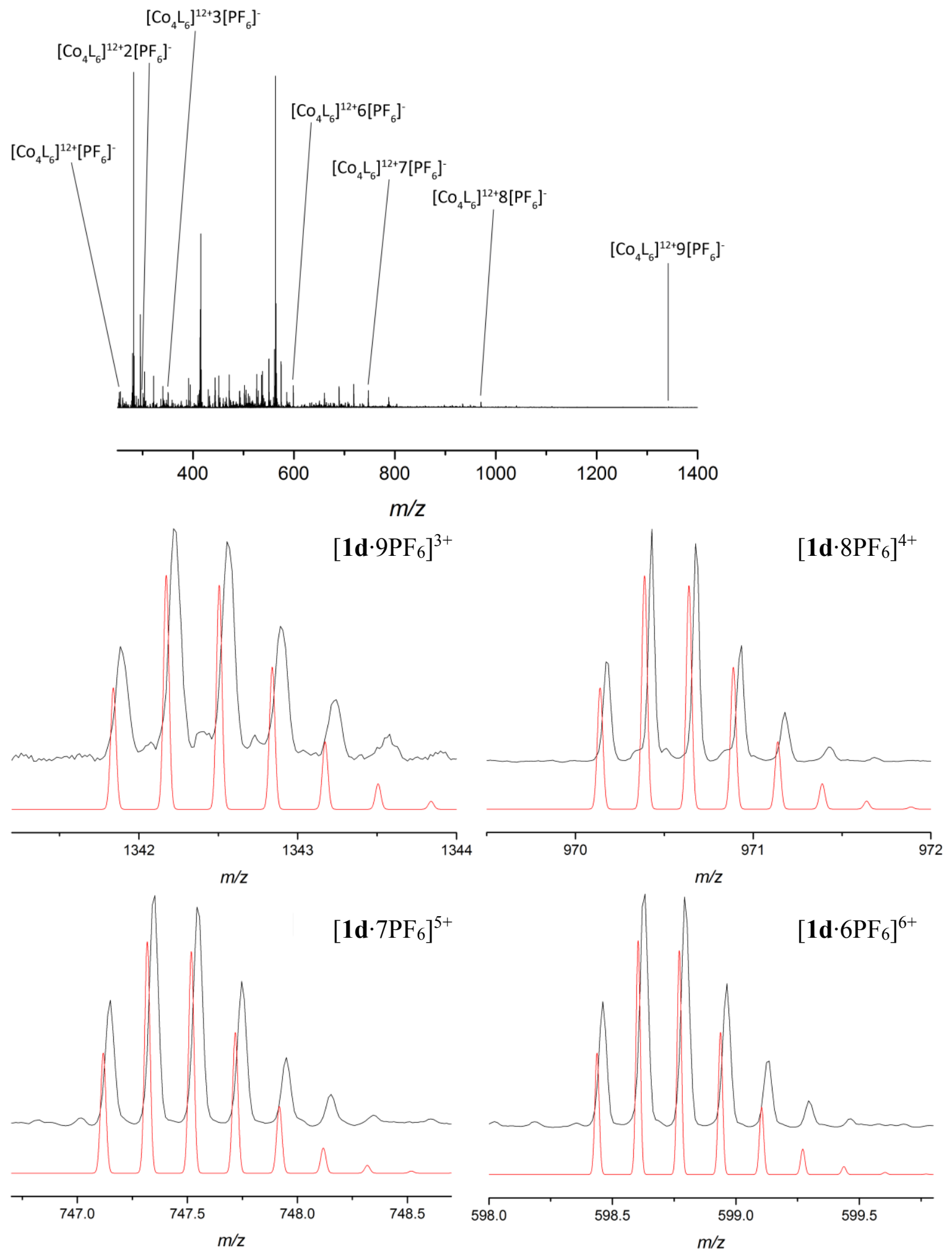

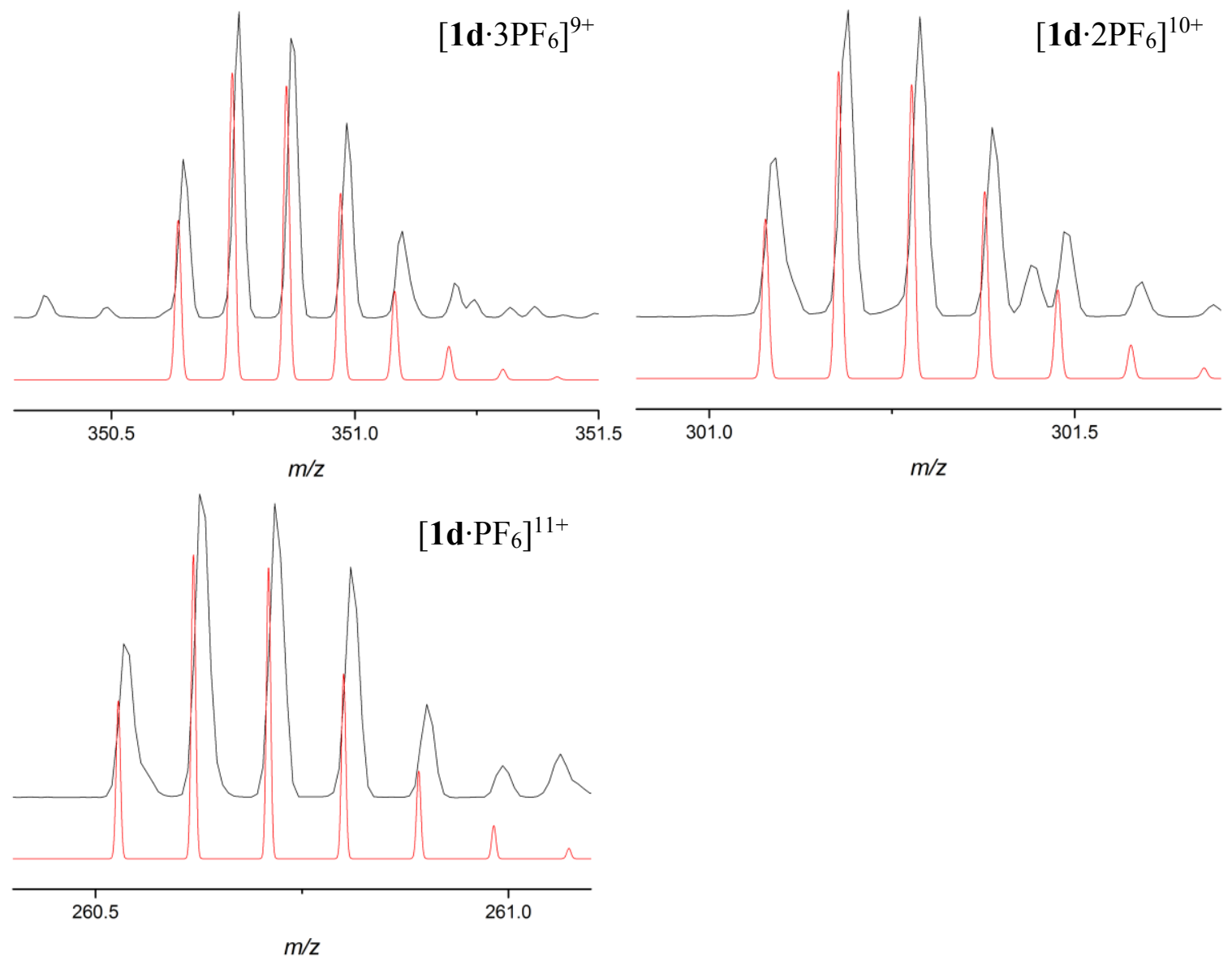

Figure S56: Mass Spectrum of $1 \mathrm{~d} \cdot 12 \mathrm{PF}_{6}$. Full spectrum (top) is shown with expansions of the labelled peaks (bottom) to demonstrate isotopic distribution. Recorded data (black) is shown against modelled data for the tetrahedron (red). 
$\mathbf{2 a} \cdot 6 \mathrm{PF}_{6}$
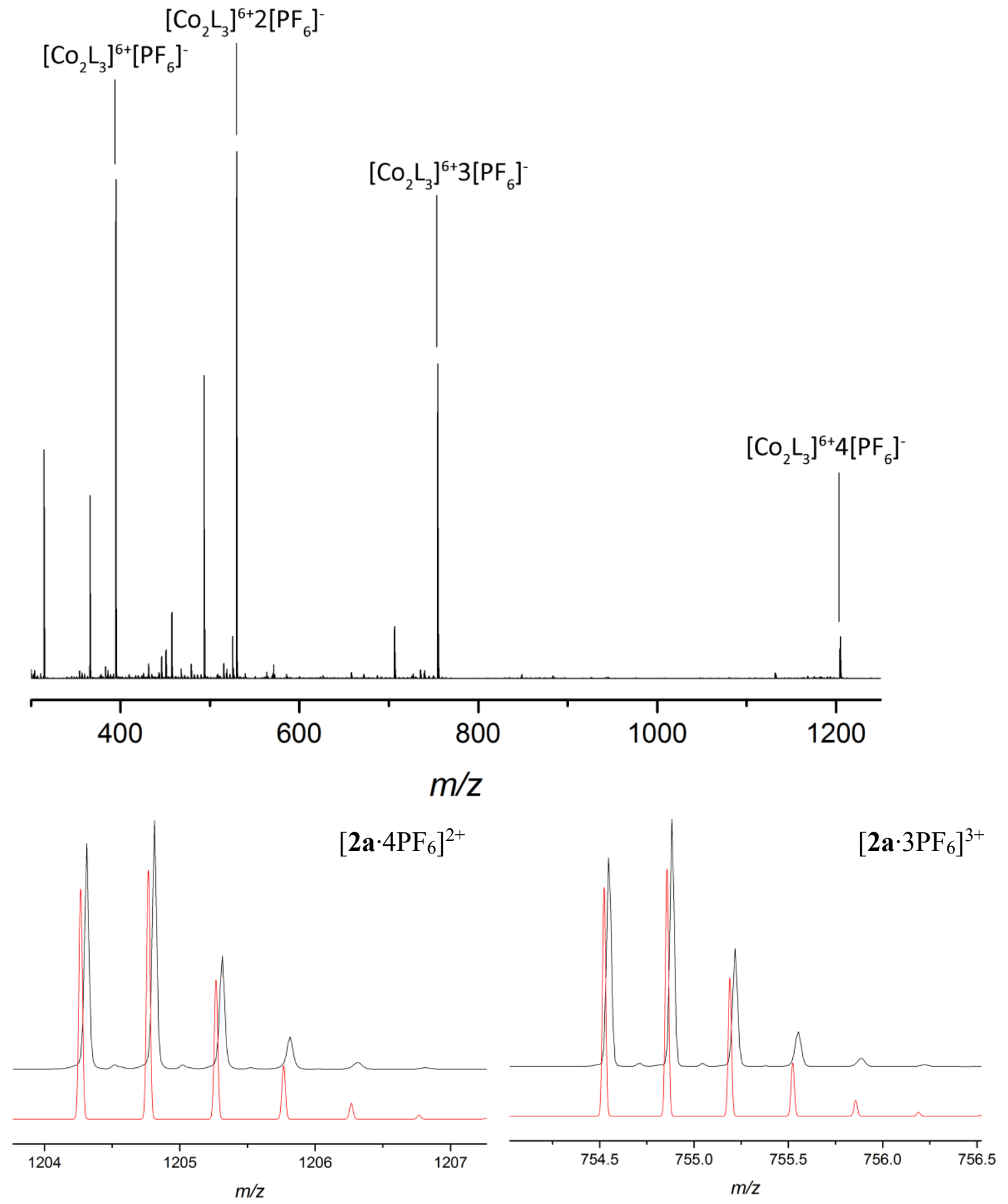

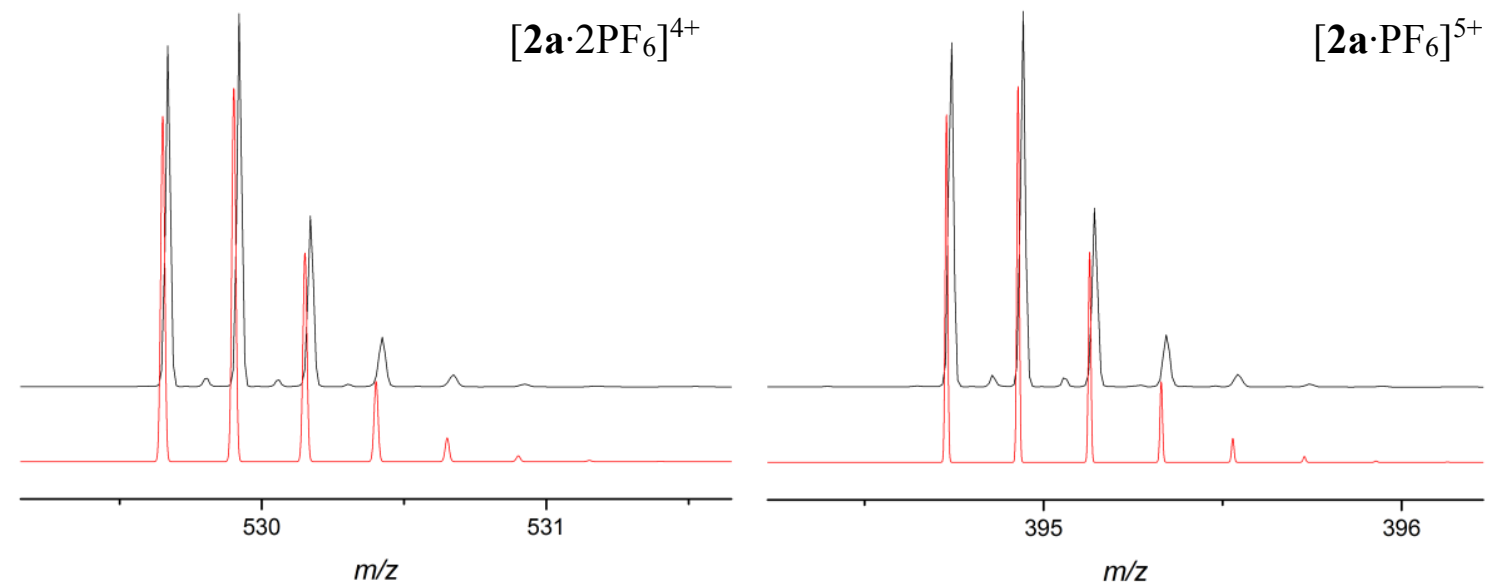

Figure S57: Mass Spectrum of $2 \mathbf{a} \cdot 6 \mathrm{PF}_{6}$. Full spectrum (top) is shown with expansions of the labelled peaks (bottom) to demonstrate isotopic distribution. Recorded data (black) is shown against modelled data for the helicate (red).

\section{2b· $6 \mathrm{PF}_{6}$}

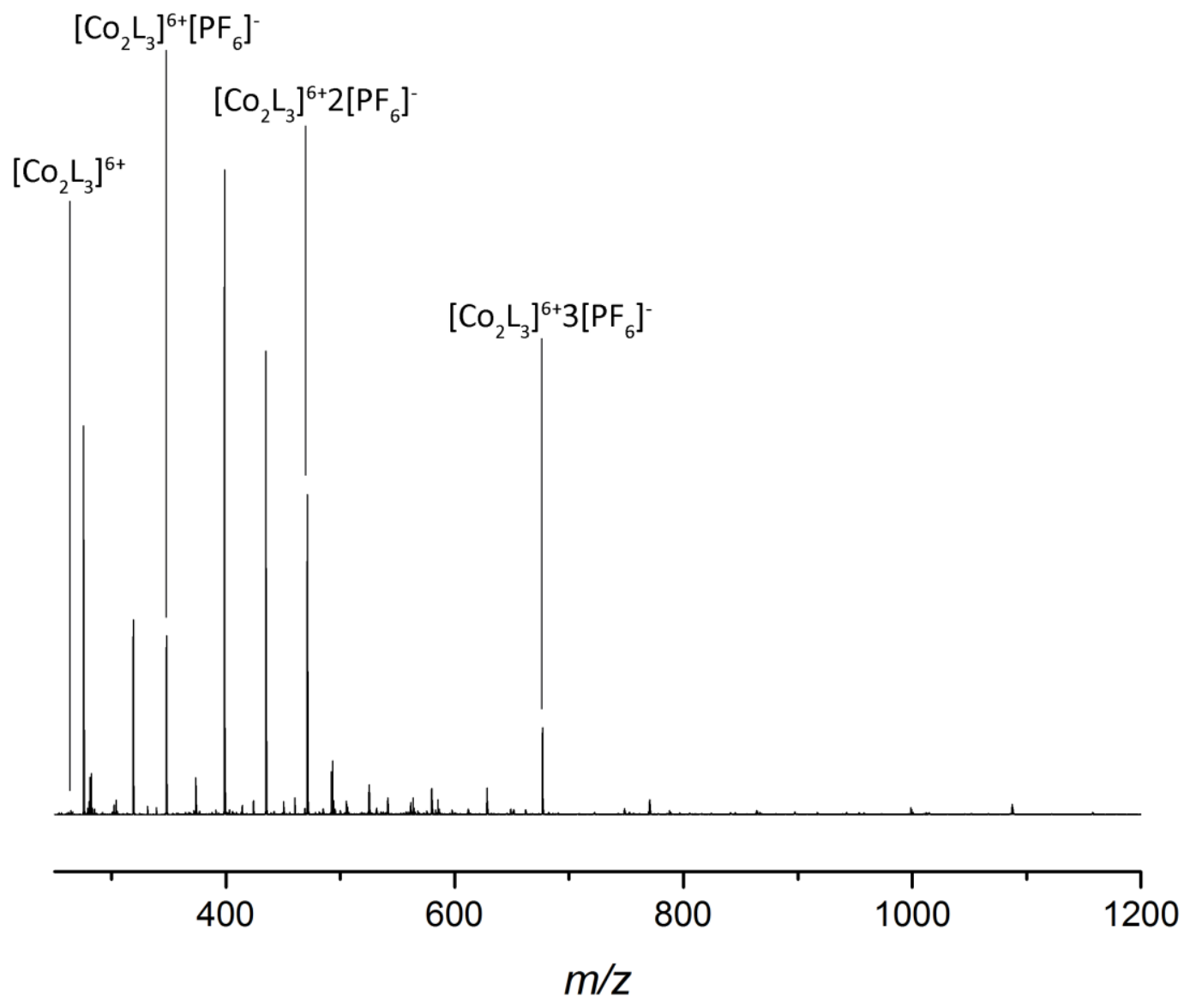



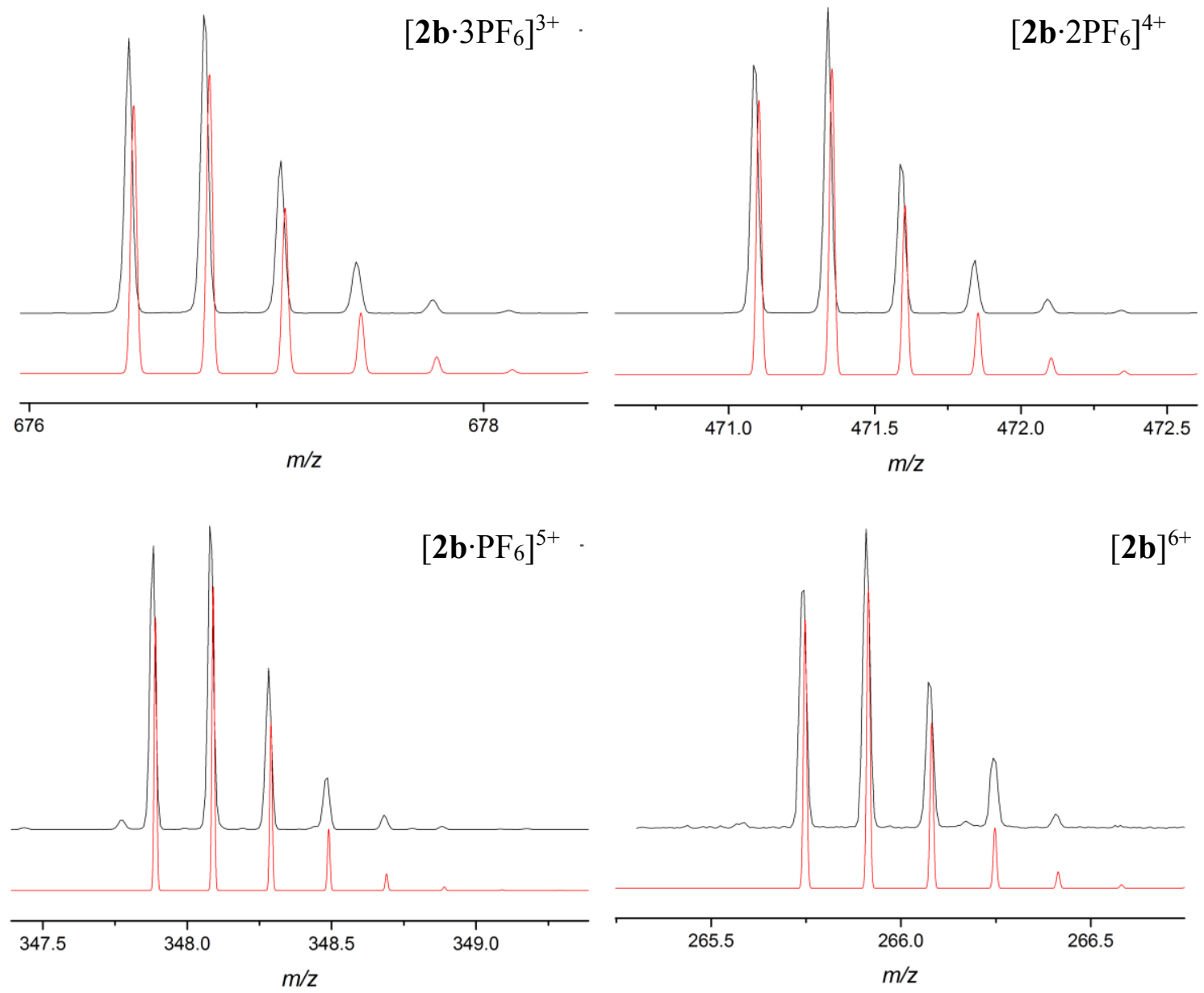

Figure S58: Mass Spectrum of $\mathbf{2 b} \cdot 6 \mathrm{PF}_{6}$. Full spectrum (top) is shown with expansions of the labelled peaks (bottom) to demonstrate isotopic distribution. Recorded data (black) is shown against modelled data for the helicate (red).

2c. $6 \mathrm{PF}_{6}$

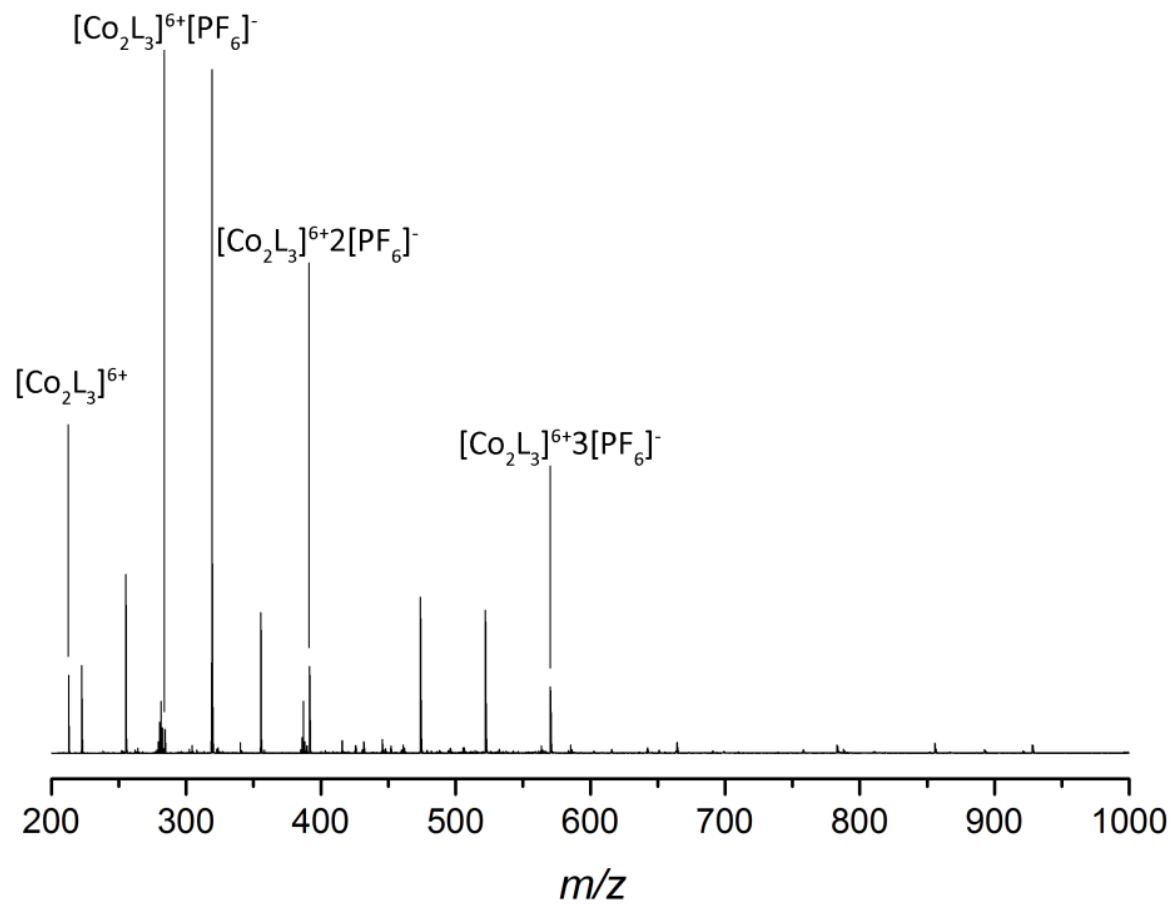



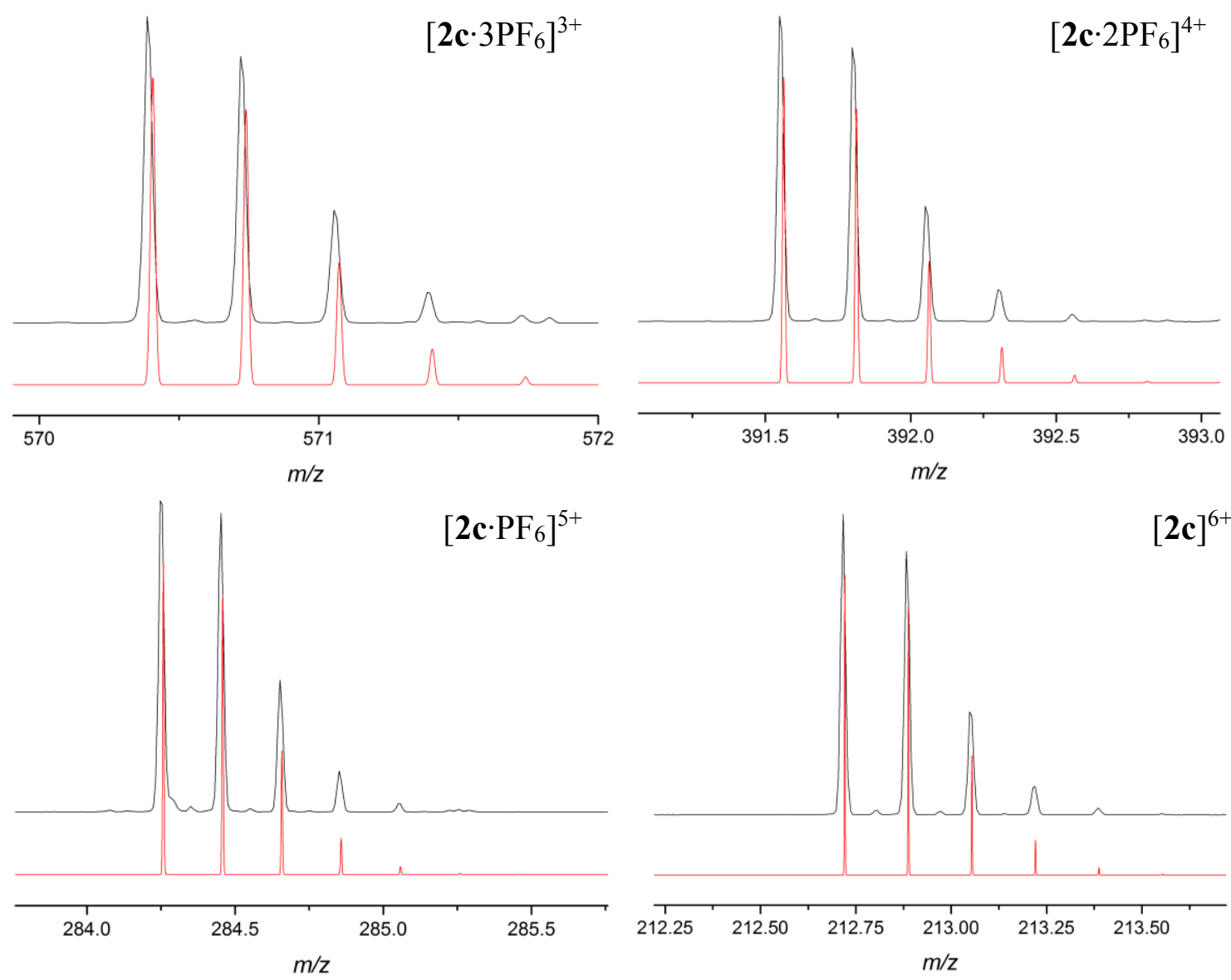

Figure S59: Mass Spectrum of 2c.6PF 6 . Full spectrum (top) is shown with expansions of the labelled peaks (bottom) to demonstrate isotopic distribution. Recorded data (black) is shown against modelled data for the helicate (red). 


\section{2d. $6 \mathrm{PF}_{6}$}
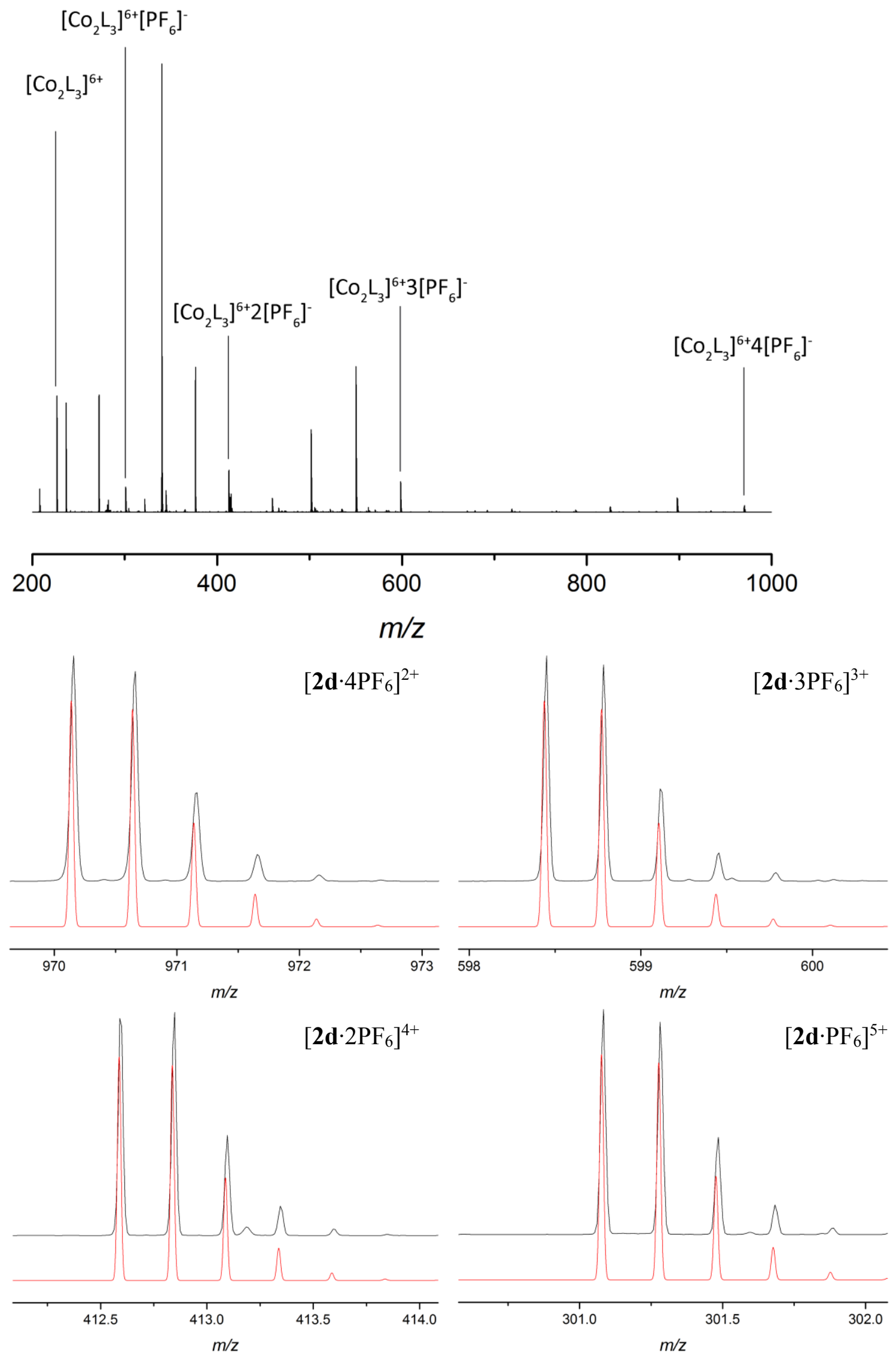


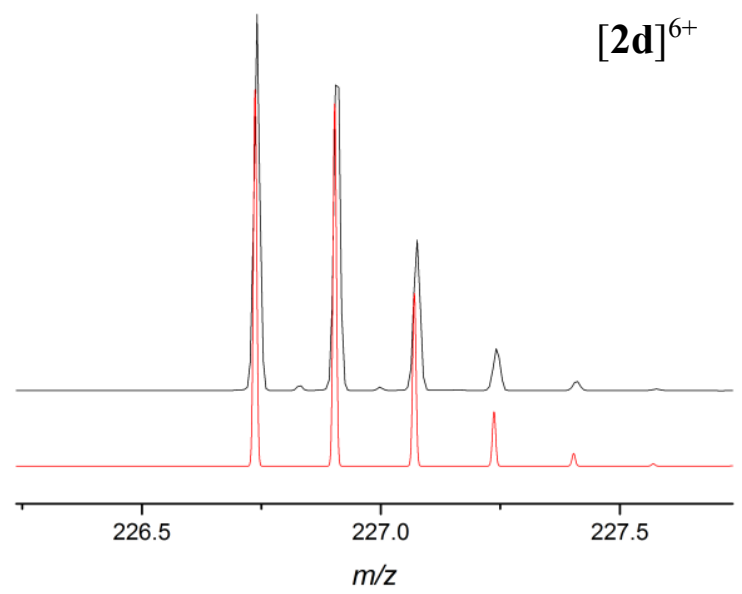

Figure S60: Mass Spectrum of $\mathbf{2 d} \cdot 6 \mathrm{PF}_{6}$. Full spectrum (top) is shown with expansions of the labelled peaks (bottom) to demonstrate isotopic distribution. Recorded data (black) is shown against modelled data for the helicate (red). 


\section{X-ray crystallography}

\section{1b·12PF 6 Crystal Data (CCDC 1425917) and Experimental}

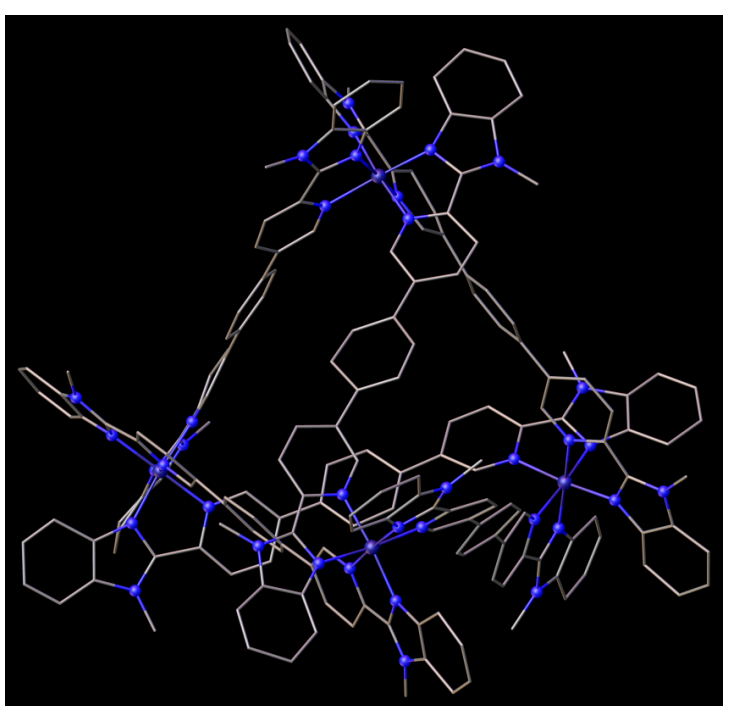

Experimental. Single red plate-shaped crystals of (PL15004) were recrystallised from a mixture of acetonitrile and diisopropyl ether by vapour diffusion. A suitable crystal $\left(0.29 \times 0.19 \times 0.06 \mathrm{~mm}^{3}\right)$ was selected and mounted on a MITIGEN holder in Paratone oil on an Agilent Technologies SuperNova diffractometer. The crystal was kept at $T=120.0 \mathrm{~K}$ during data collection. Using Olex2, ${ }^{[3]}$ the structure was solved with the ShelXT structure solution program, ${ }^{[4]}$ using the Direct Methods solution method. The model was refined with version of ShelXL using Least Squares minimisation. ${ }^{[5]}$

Crystal Data. $\mathrm{C}_{250} \mathrm{H}_{231} \mathrm{Co}_{4} \mathrm{~F}_{72} \mathrm{~N}_{65} \mathrm{P}_{12}, \quad M_{r}=6121.34$, monoclinic, $\mathrm{P} 2_{1} / \mathrm{c}$ (No. 14), $a=18.6723$ (3) $\AA, b=38.7772(8)$ $\AA, c=38.7947(6) \AA, \beta=92.0840(16)^{\circ}, \alpha=\gamma=90^{\circ}, V=$ 28071.1(9) $\AA^{3}, T=120.0 \mathrm{~K}, Z=4, Z^{\prime}=1, \mu\left(\mathrm{CuK}_{\alpha}\right)=3.447$, 116463 reflections measured, 15031 unique $\left(R_{\text {int }}=0.0805\right)$ which were used in all calculations. The final $w R_{2}$ was 0.4577 (all data) and $R_{l}$ was 0.1725 (I > 2(I)).

\begin{tabular}{|c|c|}
\hline Compound & PL15004 \\
\hline Formula & $\mathrm{C}_{250} \mathrm{H}_{231} \mathrm{Co}_{4} \mathrm{~F}_{72} \mathrm{~N}_{65} \mathrm{P}_{12}$ \\
\hline$D_{\text {calc. }} / \mathrm{g} \mathrm{cm}^{-3}$ & 1.448 \\
\hline$\mu / \mathrm{mm}^{-1}$ & 3.447 \\
\hline Formula Weight & 6121.34 \\
\hline Colour & red \\
\hline Shape & plate \\
\hline Max Size/mm & 0.29 \\
\hline Mid Size/mm & 0.19 \\
\hline Min Size/mm & 0.06 \\
\hline$T / \mathrm{K}$ & 120.0 \\
\hline Crystal System & monoclinic \\
\hline Space Group & $\mathrm{P} 2{ }_{1} / \mathrm{c}$ \\
\hline$a / \AA$ & $18.6723(3)$ \\
\hline$b / \AA$ & $38.7772(8)$ \\
\hline$c / \AA$ & $38.7947(6)$ \\
\hline$\alpha /^{\circ}$ & 90 \\
\hline$\beta /^{\circ}$ & $92.0840(16)$ \\
\hline$\left.\gamma\right|^{\circ}$ & 90 \\
\hline $\mathrm{V} / \AA^{3}$ & 28071.1(9) \\
\hline$Z$ & 4 \\
\hline$Z^{\prime}$ & 1 \\
\hline$\Theta_{\min } /^{\circ}$ & 3.224 \\
\hline$\left.\Theta_{\max }\right|^{\circ}$ & 38.078 \\
\hline Measured Refl. & 116463 \\
\hline Independent Refl. & 15031 \\
\hline Reflections Used & 10625 \\
\hline$R_{\text {int }}$ & 0.0805 \\
\hline Parameters & 1351 \\
\hline Restraints & 1200 \\
\hline Largest Peak & 1.571 \\
\hline Deepest Hole & -2.161 \\
\hline GooF & 3.301 \\
\hline$w R_{2}$ (all data) & 0.4577 \\
\hline$w R_{2}$ & 0.4398 \\
\hline$R_{l}$ (all data) & 0.2040 \\
\hline$R_{1}$ & 0.1725 \\
\hline
\end{tabular}


Experimental Extended. A red plate-shaped crystal with dimensions $0.29 \times 0.19 \times 0.06 \mathrm{~mm}^{3}$ was mounted on on a MITIGEN holder in Paratone oil. Data were collected using a Agilent Technologies SuperNova diffractometer equipped with an Oxford Cryosystems Cryostream 700+ low-temperature apparatus operating at $T=120.0 \mathrm{~K}$.

Data were measured using $\omega$ scans scans of $0.3^{\circ}$ per frame for $7.5 \mathrm{~s}$ using $\mathrm{CuK}_{\alpha}$ radiation (sealed X-ray tube, $50 \mathrm{kV}, 0.8 \mathrm{~mA}$ ). The total number of runs and images was based on the strategy calculation from the program CrysAlisPro (Agilent, V1.171.37.35e, 2014). The actually achieved resoltion was $\Theta=38.078$.

Cell parameters were retrieved using the CrysAlisPro (Agilent, V1.171.37.35e, 2014) software and refined using CrysAlisPro (Agilent, V1.171.37.35e, 2014) on 32595 reflections.

Data reduction was performed using the CrysAlisPro (Agilent, V1.171.37.35e, 2014) software, which corrects for Lorentz polarisation. The final completeness is 99.90 out to 38.078 in $\Theta$. The absorption coefficient $(\mu)$ of this material is 3.447 and the minimum and maximum transmissions are 0.910 and 0.978 .

The structure was solved by Direct Methods using the ShelXT structure solution program and refined by Least Squares using version of ShelXL. ${ }^{[4-5]}$

The structure was solved in the space group $\mathrm{P} 2{ }_{1} / \mathrm{c}$ (\# 14). All non-hydrogen atoms were refined anisotropically. Hydrogen atom positions were calculated geometrically and refined using the riding model.

The compound crystallized as relatively large well-formed block crystals with sharp edges and corners. However, when a sample of crystals was removed by pipette and transferred to a double well microscope slide they began to decompose instantly. A block crystal was scooped up in Paratone oil and transferred to the diffractometer with the absolute minimum of delay. Despite this, diffraction quality was very poor. A data collection strategy, with copper radiation, taking several days was used. The resolution of the data set was cut at $1.25 \AA$ during data processing. The tetrahedral structure was found easily by direct methods as were around half of the counterions. The remaining counterions were established by means of successive difference Fourier map analysis. Only the Co centres of the tetrahedron could be refined anisotropically. Some of the 5- and 6membered rings were modelled using idealized pentagonal and hexagonal constraints to control their geometry. These constraints were not removed. Similarly, all N-CH3 distances were restrained to be similar. Most of the counterions were found by structure solution. The remainder were established by difference map analysis. Some counterions were sufficiently well behaved to be refined using an isotropic model. The geometries of all counterions from P6 to P12 were restrained to be similar to that of P1, and isotropic displacement parameters of the fluorine atoms in these counterions were not refined. Two of the counterions, P11 and P12, could only be found in part by difference map analysis; it was clear that the counterion was present but not all fluorine atoms could be located. In these cases a model from Cambridge Structural Database entry BOBHAD was used to generate the remaining fluorine sites using the FRAG/FEND combination in ShelXL. Counterion P8 was modelled as disordered over two sites, since this was clear from difference map analysis. The solvent masking routine in Olex2 was then used to remove all other significant residual electron density. A total of 1831 electrons per unit cell were masked. This corresponds to approximately 29 molecules of acetonitrile per asymmetric unit. This solvent is included in the total chemical formula and all derived values. Doing so triggers checkCIF alerts, which should be ignored. The refinement has not converged sufficiently to allow for optimisation of the ShelXL weighting scheme. The structure is sufficient only for confirmation of connectivity and molecular shape. 


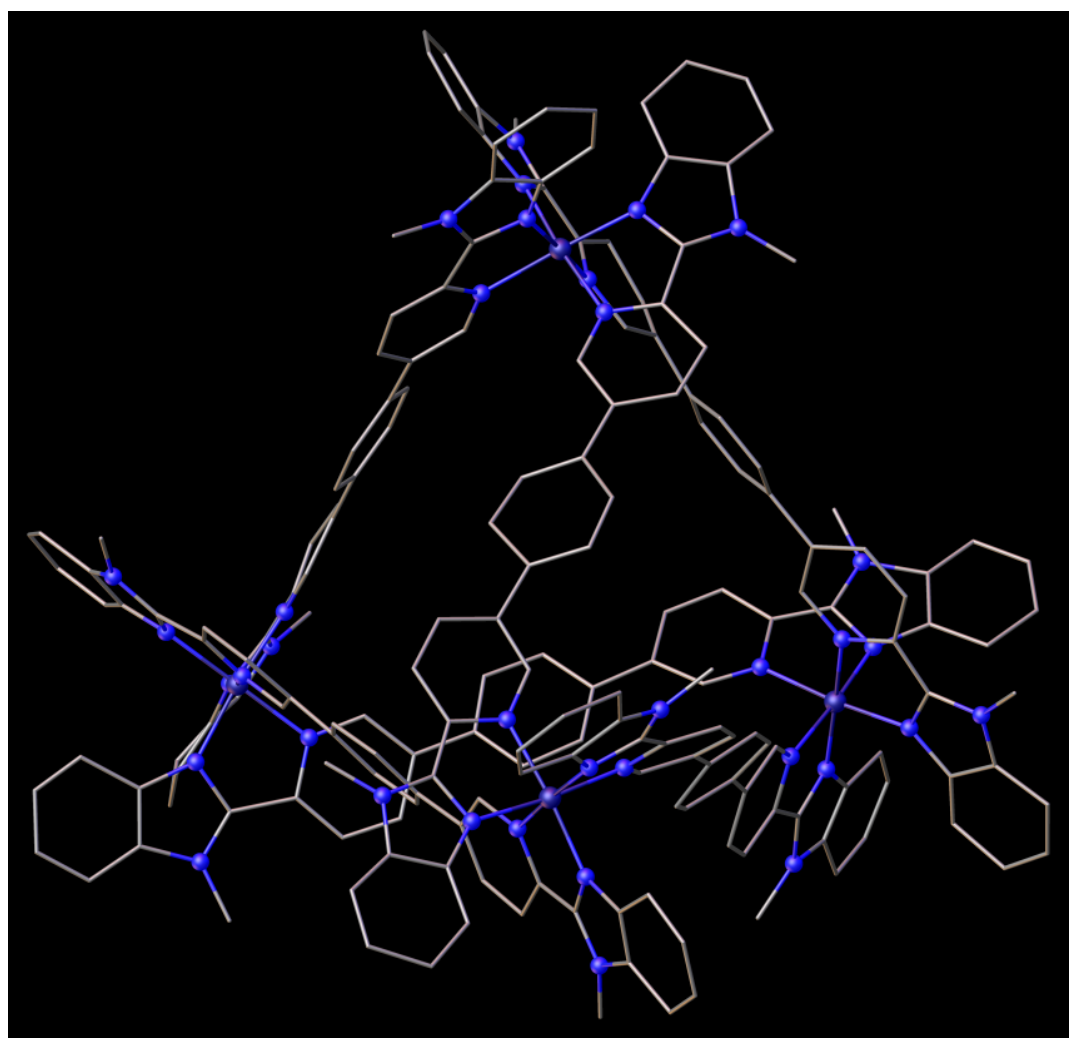

Figure S60: The tetrahedral structure of pl15004.

\section{Reflection Statistics}

$\begin{array}{llll}\text { Total reflections } & 116463 & \text { Unique reflections } & 15031 \\ \text { Completeness } & 1.0 & \text { Mean I/ } & 15.42 \\ \text { Max hkl collected } & (14,31,31) & \text { Min hkl collected } & (-14,-31,-31) \\ \text { Max hkl used } & (14,31,31) & \text { Min hkl used } & (-14,0,0) \\ \text { Lim d max } & 100.0 & \text { Lim d min } & 0.77 \\ \text { d max used } & 13.71 & \text { d min used } & 1.25 \\ \text { Friedel pairs } & 21862 & \text { Friedel pairs merged } & 1 \\ \text { Inconsistent equivalents } & 17 & R_{\text {int }} & 0.0805 \\ R_{\square} & 0.0449 & \text { Intensity transformed } & 0 \\ \text { Omitted reflections } & 0 & \text { Omitted by user } & 0 \\ \text { Multiplicity } & (18919,15421,8593,5569, \text { ReflectionAPotMax } & 20 \\ & 2195,853,256,84,10) & & \\ \text { Removed } & \text { Systematic } 1627 & \text { Filtered Off } & 0 \\ \text { Absences } & & \end{array}$

Table S2: Fractional Atomic Coordinates $\left(\times 10^{4}\right)$ and Equivalent Isotropic Displacement Parameters $\left(A^{2} \times 10^{3}\right)$ for PL15004. $U_{e q}$ is defined as $1 / 3$ of of the trace of the orthogonalised $U_{i j}$.

\begin{tabular}{lllll}
\hline Atom & \multicolumn{1}{c}{$\mathbf{x}$} & \multicolumn{1}{c}{$\mathbf{y}$} & \multicolumn{1}{c}{$\mathbf{z}$} & \multicolumn{1}{c}{$\boldsymbol{U}_{\boldsymbol{e q}}$} \\
\hline Co1 & $8255.0(18)$ & $8366.1(9)$ & $8862.6(8)$ & $29.7(12)$ \\
Co2 & $8830.7(19)$ & $5895.2(10)$ & $6521.4(8)$ & $43.9(13)$ \\
Co3 & $3181.5(18)$ & $5991.9(9)$ & $8445.7(8)$ & $33.3(12)$ \\
Co4 & $3989.7(18)$ & $8354.3(10)$ & $6037.3(8)$ & $36.9(12)$ \\
N1 & $8634(9)$ & $8235(5)$ & $8410(4)$ & $35(5)$ \\
N2 & $8618(8)$ & $8836(4)$ & $8724(4)$ & $27(5)$ \\
N3 & $9064(9)$ & $9136(5)$ & $8336(4)$ & $34(5)$ \\
N4 & $8898(8)$ & $6026(4)$ & $7011(4)$ & $25(5)$
\end{tabular}




\begin{tabular}{|c|c|c|c|c|}
\hline Atom & $\mathbf{x}$ & $\mathbf{y}$ & $\mathbf{Z}$ & $U_{e q}$ \\
\hline N5 & $9037(9)$ & $5453(5)$ & $6713(5)$ & $48(6)$ \\
\hline N6 & 8853(9) & $5113(5)$ & $7177(4)$ & $43(6)$ \\
\hline N7 & $7970(10)$ & $7895(5)$ & $8970(4)$ & $41(6)$ \\
\hline N8 & $9133(8)$ & $8220(4)$ & $9093(4)$ & $18(5)$ \\
\hline N9 & $9798(10)$ & $7786(5)$ & $9279(4)$ & $42(6)$ \\
\hline N11 & $2230(9)$ & $6182(4)$ & $8496(4)$ & $31(5)$ \\
\hline N12 & $1518(10)$ & $6626(5)$ & $8628(4)$ & $37(5)$ \\
\hline N13 & $7332(9)$ & $8482(4)$ & $8653(4)$ & $28(5)$ \\
\hline N14 & $7809(10)$ & $8520(4)$ & $9271(4)$ & $36(5)$ \\
\hline N15 & $6850(10)$ & $8718(4)$ & $9513(5)$ & $41(5)$ \\
\hline N16 & 4952(9) & $8453(4)$ & $6242(4)$ & $26(5)$ \\
\hline N17 & $4501(10)$ & $8471(4)$ & $5616(4)$ & $37(5)$ \\
\hline N18 & $5517(10)$ & $8588(5)$ & $5373(5)$ & $50(6)$ \\
\hline N19 & $7827(10)$ & $5764(5)$ & $6544(5)$ & 51(6) \\
\hline N21 & $8000(10)$ & $5435(5)$ & $5647(6)$ & $116(9)$ \\
\hline C104 & $8054(8)$ & $5551(5)$ & $5994(5)$ & $94(10)$ \\
\hline N20 & $8724(9)$ & $5720(4)$ & $6045(4)$ & $48(6)$ \\
\hline C97 & $9085(8)$ & $5708(5)$ & $5729(5)$ & $64(8)$ \\
\hline C102 & $8637(12)$ & $5532(5)$ & $5483(3)$ & $98(11)$ \\
\hline N23 & $3335(9)$ & $5852(4)$ & $8913(4)$ & $32(5)$ \\
\hline N24 & $4056(10)$ & $5634(5)$ & $9324(5)$ & $50(6)$ \\
\hline N25 & $8627(11)$ & $6370(5)$ & $6344(4)$ & $42(6)$ \\
\hline N27 & $10538(10)$ & $6467(5)$ & $6308(5)$ & $55(6)$ \\
\hline C137 & $9830(13)$ & $6367(6)$ & $6354(6)$ & $42(7)$ \\
\hline N26 & $9802(10)$ & $6041(5)$ & $6466(5)$ & $52(6)$ \\
\hline C135 & 10932(9) & $6181(3)$ & $6407(4)$ & $47(7)$ \\
\hline C134 & $11668(8)$ & $6125(3)$ & $6422(3)$ & $51(7)$ \\
\hline C133 & $11942(6)$ & $5813(4)$ & $6543(3)$ & $38(7)$ \\
\hline C132 & $11479(8)$ & $5555(3)$ & $6648(3)$ & $31(6)$ \\
\hline C131 & $10743(7)$ & $5611(4)$ & $6632(3)$ & $41(7)$ \\
\hline C130 & $10470(6)$ & $5924(4)$ & $6512(4)$ & $42(7)$ \\
\hline N28 & $4216(9)$ & $7875(4)$ & $5956(4)$ & $22(5)$ \\
\hline N29 & $3103(7)$ & $8236(4)$ & $5797(3)$ & $40(5)$ \\
\hline C156 & $3086(8)$ & $7875(4)$ & $5733(4)$ & $56(8)$ \\
\hline N30 & 2374(9) & $7784(4)$ & $5629(4)$ & $65(7)$ \\
\hline C154 & $1951(6)$ & $8088(5)$ & $5629(5)$ & $84(10)$ \\
\hline C149 & $2402(9)$ & $8367(3)$ & $5732(4)$ & $65(8)$ \\
\hline $\mathrm{C} 150$ & $2087(17)$ & $8689(8)$ & $5749(7)$ & $100(11)$ \\
\hline C151 & $1320(20)$ & $8732(10)$ & $5689(9)$ & $138(14)$ \\
\hline C152 & $1100(20)$ & $8468(12)$ & $5466(11)$ & $162(16)$ \\
\hline C153 & $1255(19)$ & $8105(10)$ & $5464(9)$ & $139(14)$ \\
\hline N31 & $3087(8)$ & $6107(5)$ & $7949(4)$ & $31(5)$ \\
\hline N32 & 2899(8) & $5549(4)$ & $8288(4)$ & $30(5)$ \\
\hline N33 & $2840(10)$ & $5192(5)$ & $7835(5)$ & $59(6)$ \\
\hline N34 & $3518(8)$ & $8245(5)$ & $6474(4)$ & $27(5)$ \\
\hline N35 & $3684(9)$ & $8823(4)$ & $6147(4)$ & $35(5)$ \\
\hline N36 & $3069(9)$ & $9163(5)$ & $6482(5)$ & $43(6)$ \\
\hline $\mathrm{C} 1$ & $8667(11)$ & $9149(6)$ & $8844(6)$ & $29(6)$ \\
\hline $\mathrm{C} 2$ & $8511(13)$ & $9303(7)$ & $9153(7)$ & $70(9)$ \\
\hline $\mathrm{C} 3$ & $8595(13)$ & $9656(7)$ & $9215(7)$ & $69(9)$ \\
\hline $\mathrm{C} 4$ & $8908(14)$ & $9859(8)$ & $8930(7)$ & $84(10)$ \\
\hline $\mathrm{C} 5$ & $9060(12)$ & $9690(7)$ & $8639(6)$ & $56(8)$ \\
\hline C6 & $8942(11)$ & $9360(6)$ & $8607(6)$ & $35(7)$ \\
\hline $\mathrm{C} 7$ & $9324(13)$ & $9246(7)$ & $8010(5)$ & $79(9)$ \\
\hline $\mathrm{C} 8$ & $8877(10)$ & $8819(6)$ & $8415(5)$ & $22(6)$ \\
\hline C9 & $8867(10)$ & $8504(5)$ & $8229(5)$ & $18(6)$ \\
\hline $\mathrm{C} 10$ & $9135(12)$ & $8441(6)$ & $7898(6)$ & $53(8)$ \\
\hline C11 & $9093(13)$ & $8110(7)$ & $7771(7)$ & $64(8)$ \\
\hline $\mathrm{C} 12$ & $8880(11)$ & $7847(6)$ & $7966(5)$ & $27(6)$ \\
\hline C13 & $8637(10)$ & $7920(5)$ & $8275(5)$ & $21(6)$ \\
\hline $\mathrm{C} 14$ & $8931(12)$ & $7486(7)$ & $7866(6)$ & $55(8)$ \\
\hline $\mathrm{C} 15$ & $8804(15)$ & $7422(9)$ & $7479(8)$ & 101(11) \\
\hline $\mathrm{C} 16$ & $8738(13)$ & $7079(7)$ & $7388(7)$ & 73(9) \\
\hline $\mathrm{C} 17$ & $8913(12)$ & $6781(7)$ & $7592(6)$ & $47(7)$ \\
\hline
\end{tabular}




\begin{tabular}{|c|c|c|c|c|}
\hline Atom & $\mathbf{x}$ & $\mathbf{y}$ & $\mathbf{z}$ & $\boldsymbol{U}_{e q}$ \\
\hline$\overline{\mathrm{C} 18}$ & $9107(13)$ & $6883(7)$ & $7944(7)$ & $71(9)$ \\
\hline C19 & $9104(13)$ & $7199(7)$ & $8074(7)$ & $70(9)$ \\
\hline $\mathrm{C} 20$ & $9066(14)$ & $5096(8)$ & $6606(8)$ & $76(9)$ \\
\hline $\mathrm{C} 21$ & $9154(13)$ & $4958(7)$ & $6273(7)$ & $77(9)$ \\
\hline $\mathrm{C} 22$ & $9153(13)$ & $4597(7)$ & $6270(7)$ & $70(9)$ \\
\hline $\mathrm{C} 23$ & $9001(14)$ & $4420(8)$ & $6566(8)$ & $81(9)$ \\
\hline $\mathrm{C} 24$ & $8850(13)$ & $4535(8)$ & $6872(7)$ & $76(9)$ \\
\hline $\mathrm{C} 25$ & $8922(14)$ & $4873(8)$ & $6860(7)$ & $76(9)$ \\
\hline $\mathrm{C} 26$ & $8638(14)$ & $5013(7)$ & $7516(5)$ & $81(9)$ \\
\hline $\mathrm{C} 27$ & $8962(11)$ & $5426(6)$ & $7053(6)$ & $34(7)$ \\
\hline $\mathrm{C} 28$ & $8930(12)$ & $5769(6)$ & $7226(6)$ & $46(7)$ \\
\hline $\mathrm{C} 29$ & $8914(9)$ & $5800(6)$ & $7604(5)$ & $22(6)$ \\
\hline $\mathrm{C} 30$ & $8916(12)$ & $6126(6)$ & $7697(6)$ & $52(8)$ \\
\hline C31 & $8921(11)$ & $6422(6)$ & $7481(6)$ & $36(7)$ \\
\hline C32 & $8919(10)$ & $6352(6)$ & $7131(6)$ & $34(7)$ \\
\hline C33 & $9810(12)$ & $8339(6)$ & $9122(5)$ & $37(7)$ \\
\hline C34 & $10162(14)$ & $8679(7)$ & $9069(6)$ & $65(8)$ \\
\hline $\mathrm{C} 35$ & $10865(16)$ & $8702(8)$ & $9120(7)$ & $81(9)$ \\
\hline C36 & $11282(18)$ & $8433(9)$ & $9247(8)$ & 109(11) \\
\hline C37 & $11007(16)$ & $8064(8)$ & $9310(7)$ & $88(10)$ \\
\hline C38 & $10230(13)$ & $8059(6)$ & $9259(6)$ & $44(7)$ \\
\hline C39 & $10001(12)$ & $7437(5)$ & $9360(6)$ & $57(8)$ \\
\hline $\mathrm{C} 40$ & $9157(11)$ & $7890(5)$ & $9187(5)$ & $15(6)$ \\
\hline C41 & $8483(11)$ & $7710(6)$ & $9152(5)$ & $24(6)$ \\
\hline $\mathrm{C} 42$ & $8344(13)$ & $7398(6)$ & $9276(6)$ & $54(8)$ \\
\hline $\mathrm{C} 43$ & $7622(16)$ & $7269(8)$ & $9205(7)$ & $94(10)$ \\
\hline $\mathrm{C} 44$ & $7128(11)$ & $7433(6)$ & $9016(5)$ & $26(6)$ \\
\hline $\mathrm{C} 45$ & $7329(12)$ & $7755(6)$ & $8910(5)$ & $36(7)$ \\
\hline C46 & $6394(14)$ & $7292(7)$ & $8947(6)$ & $58(8)$ \\
\hline $\mathrm{C} 47$ & $6237(13)$ & $6947(6)$ & $8965(5)$ & $53(8)$ \\
\hline $\mathrm{C} 48$ & $5566(12)$ & $6826(6)$ & $8908(5)$ & $37(7)$ \\
\hline C49 & $4969(12)$ & $7041(6)$ & $8787(5)$ & $36(7)$ \\
\hline C50 & $5166(14)$ & $7390(7)$ & $8765(6)$ & $72(9)$ \\
\hline C51 & $5845(14)$ & $7523(7)$ & $8875(6)$ & $67(9)$ \\
\hline C52 & $1492(13)$ & $6064(7)$ & $8490(5)$ & $44(7)$ \\
\hline C53 & $1229(12)$ & $5734(6)$ & $8416(5)$ & $40(7)$ \\
\hline C54 & $509(14)$ & $5716(7)$ & $8411(6)$ & $58(8)$ \\
\hline C55 & 77(14) & $6000(7)$ & $8477(6)$ & $65(8)$ \\
\hline C56 & $312(15)$ & $6312(8)$ & $8572(6)$ & $81(9)$ \\
\hline C57 & $1115(13)$ & $6339(6)$ & $8555(5)$ & $41(7)$ \\
\hline C58 & $1315(13)$ & $6981(5)$ & $8679(6)$ & $77(9)$ \\
\hline C59 & $2218(12)$ & $6505(6)$ & $8591(5)$ & $32(7)$ \\
\hline C61 & 2995(8) & $7021(4)$ & $8720(4)$ & $73(9)$ \\
\hline C62 & $3686(9)$ & $7147(3)$ & $8775(4)$ & $83(9)$ \\
\hline C64 & $4272(7)$ & $6932(4)$ & $8731(4)$ & $32(7)$ \\
\hline C63 & $4165(6)$ & $6591(3)$ & $8631(3)$ & $24(6)$ \\
\hline N10 & $3474(8)$ & $6464(3)$ & $8576(3)$ & $33(5)$ \\
\hline C60 & $2889(6)$ & $6679(4)$ & $8621(3)$ & $31(7)$ \\
\hline C65 & $8005(13)$ & $8580(6)$ & $9622(6)$ & $44(7)$ \\
\hline C66 & $8637(12)$ & $8495(5)$ & $9810(6)$ & $38(7)$ \\
\hline C67 & $8551(14)$ & $8566(6)$ & $10155(6)$ & $56(8)$ \\
\hline C68 & $7977(14)$ & $8671(6)$ & $10316(7)$ & $53(8)$ \\
\hline C69 & $7389(16)$ & $8747(7)$ & $10154(7)$ & $84(10)$ \\
\hline $\mathrm{C} 70$ & $7434(15)$ & $8686(6)$ & $9762(7)$ & $61(8)$ \\
\hline C71 & $6136(12)$ & $8833(7)$ & $9588(7)$ & $92(10)$ \\
\hline $\mathrm{C} 72$ & $7182(13)$ & $8617(6)$ & $9241(6)$ & $37(7)$ \\
\hline C73 & $6847(13)$ & $8571(6)$ & $8886(6)$ & $47(7)$ \\
\hline C74 & $6157(12)$ & $8666(5)$ & $8794(6)$ & $39(7)$ \\
\hline $\mathrm{C} 75$ & $5980(16)$ & $8634(7)$ & $8433(7)$ & $82(9)$ \\
\hline C76 & $6475(15)$ & $8542(6)$ & $8201(6)$ & $61(8)$ \\
\hline C77 & $7130(11)$ & $8461(5)$ & $8308(5)$ & $22(6)$ \\
\hline C78 & $6263(12)$ & $8532(5)$ & $7820(4)$ & $63(8)$ \\
\hline C79 & $5564(10)$ & $8465(5)$ & $7705(5)$ & $88(10)$ \\
\hline $\mathrm{C} 80$ & $5390(8)$ & $8462(5)$ & $7353(5)$ & $133(13)$ \\
\hline
\end{tabular}




\begin{tabular}{|c|c|c|c|c|}
\hline Atom & $\mathbf{x}$ & $\mathbf{y}$ & $\mathbf{z}$ & $U_{e q}$ \\
\hline$\overline{\mathrm{C} 81}$ & $5915(12)$ & $8527(5)$ & $7117(4)$ & $60(8)$ \\
\hline $\mathrm{C} 82$ & $6615(11)$ & $8594(5)$ & $7232(5)$ & $145(14)$ \\
\hline C83 & $6789(8)$ & $8597(5)$ & $7584(6)$ & $129(13)$ \\
\hline C84 & $4332(12)$ & $8489(5)$ & $5268(6)$ & $31(7)$ \\
\hline $\mathrm{C} 85$ & $3730(14)$ & $8453(6)$ & $5062(6)$ & $58(8)$ \\
\hline C86 & $3770(13)$ & $8476(5)$ & $4696(6)$ & $42(7)$ \\
\hline $\mathrm{C} 87$ & $4432(12)$ & $8541(5)$ & $4578(6)$ & $41(7)$ \\
\hline C88 & $5061(13)$ & $8594(5)$ & $4764(6)$ & $44(7)$ \\
\hline C89 & $4970(13)$ & $8559(6)$ & $5131(6)$ & $42(7)$ \\
\hline C90 & $6279(11)$ & $8583(6)$ & $5325(6)$ & $69(9)$ \\
\hline C91 & $5192(15)$ & $8540(6)$ & $5660(7)$ & $60(8)$ \\
\hline C92 & $5471(12)$ & $8521(5)$ & $6038(5)$ & $21(6)$ \\
\hline C93 & $6124(14)$ & $8570(6)$ & $6134(6)$ & $56(8)$ \\
\hline C94 & $6333(14)$ & $8589(6)$ & $6505(6)$ & $65(8)$ \\
\hline C95 & $5780(13)$ & $8503(6)$ & $6737(6)$ & $45(7)$ \\
\hline C96 & $5100(12)$ & $8463(5)$ & $6594(5)$ & $33(7)$ \\
\hline $\mathrm{C} 101$ & $8860(30)$ & $5443(11)$ & $5136(11)$ & $183(18)$ \\
\hline $\mathrm{C} 100$ & $9560(20)$ & $5539(10)$ & $5125(10)$ & $144(14)$ \\
\hline C99 & $10015(17)$ & $5695(7)$ & $5346(8)$ & $88(10)$ \\
\hline C98 & $9759(13)$ & $5791(6)$ & $5676(7)$ & $65(8)$ \\
\hline C103 & $7490(20)$ & $5254(11)$ & $5431(10)$ & $220(20)$ \\
\hline $\mathrm{C} 105$ & $7530(20)$ & $5571(9)$ & $6270(9)$ & $114(12)$ \\
\hline C106 & $6980(20)$ & $5373(11)$ & $6261(10)$ & $158(16)$ \\
\hline C107 & $6520(19)$ & $5440(8)$ & $6572(9)$ & $121(12)$ \\
\hline $\mathrm{C} 108$ & $6828(15)$ & $5597(7)$ & $6852(7)$ & $70(9)$ \\
\hline C109 & $7420(12)$ & $5772(6)$ & $6828(6)$ & $40(7)$ \\
\hline C110 & $6353(10)$ & $5661(4)$ & $7168(4)$ & $62(8)$ \\
\hline C111 & $6726(7)$ & $5712(4)$ & $7481(5)$ & $77(9)$ \\
\hline C112 & $6359(10)$ & $5725(4)$ & $7786(4)$ & $75(9)$ \\
\hline C113 & $5618(10)$ & $5687(4)$ & $7778(4)$ & $71(9)$ \\
\hline C114 & $5245(7)$ & $5636(4)$ & $7466(5)$ & $82(9)$ \\
\hline C115 & $5612(10)$ & $5623(4)$ & $7161(4)$ & $80(9)$ \\
\hline C116 & $3000(12)$ & $5859(5)$ & $9232(5)$ & $27(6)$ \\
\hline C117 & $2300(14)$ & $5954(6)$ & $9295(7)$ & $69(9)$ \\
\hline C118 & $2125(15)$ & $5932(6)$ & $9677(7)$ & $71(9)$ \\
\hline C119 & $2578(15)$ & $5772(7)$ & $9894(7)$ & $72(9)$ \\
\hline C120 & $3250(13)$ & $5657(6)$ & $9827(6)$ & $51(8)$ \\
\hline $\mathrm{C} 121$ & $3431(13)$ & $5710(6)$ & $9469(6)$ & $41(7)$ \\
\hline $\mathrm{C} 122$ & $4704(12)$ & $5511(7)$ & $9513(6)$ & $82(9)$ \\
\hline C123 & $3970(12)$ & $5720(5)$ & $8981(5)$ & $31(6)$ \\
\hline C124 & $4473(8)$ & $5699(4)$ & $8717(3)$ & $53(8)$ \\
\hline $\mathrm{C} 125$ & $5159(8)$ & $5560(4)$ & $8717(3)$ & $73(9)$ \\
\hline C126 & $5543(6)$ & $5562(4)$ & $8416(5)$ & $77(9)$ \\
\hline C127 & $5240(7)$ & $5703(4)$ & $8116(3)$ & $46(7)$ \\
\hline C128 & $4554(7)$ & $5842(3)$ & $8115(3)$ & $39(7)$ \\
\hline N22 & $4171(6)$ & $5840(3)$ & $8416(4)$ & $31(5)$ \\
\hline C136 & $10808(12)$ & $6799(5)$ & $6219(6)$ & $56(8)$ \\
\hline C138 & $9168(12)$ & $6572(6)$ & $6286(5)$ & $28(6)$ \\
\hline C139 & $9142(13)$ & $6899(6)$ & $6191(5)$ & $39(7)$ \\
\hline $\mathrm{C} 140$ & $8474(15)$ & $7019(7)$ & $6155(6)$ & $67(9)$ \\
\hline $\mathrm{C} 141$ & $7867(13)$ & $6830(6)$ & $6185(6)$ & $44(7)$ \\
\hline $\mathrm{C} 142$ & $7985(12)$ & $6498(6)$ & $6295(5)$ & $36(7)$ \\
\hline C143 & $7120(7)$ & $6971(5)$ & $6098(4)$ & $50(7)$ \\
\hline C148 & $6994(8)$ & $7321(4)$ & $6149(4)$ & $76(9)$ \\
\hline C147 & $6309(10)$ & $7454(3)$ & $6092(4)$ & $78(9)$ \\
\hline C146 & $5750(7)$ & $7238(4)$ & $5985(4)$ & $43(7)$ \\
\hline $\mathrm{C} 145$ & $5876(8)$ & $6889(4)$ & $5935(4)$ & $49(7)$ \\
\hline $\mathrm{C} 144$ & $6561(9)$ & $6755(3)$ & $5991(4)$ & $75(9)$ \\
\hline $\mathrm{C} 155$ & 2101(14) & $7438(5)$ & $5576(7)$ & $87(10)$ \\
\hline C157 & $3696(13)$ & $7677(7)$ & $5791(6)$ & $55(8)$ \\
\hline $\mathrm{C} 158$ & $3803(15)$ & $7389(8)$ & $5658(7)$ & $78(9)$ \\
\hline C159 & $4440(20)$ & $7211(11)$ & $5745(9)$ & $145(14)$ \\
\hline $\mathrm{C} 160$ & $5053(12)$ & $7390(6)$ & $5922(6)$ & $42(7)$ \\
\hline $\mathrm{C} 161$ & $4849(12)$ & $7719(6)$ & $6019(5)$ & $37(7)$ \\
\hline
\end{tabular}




\begin{tabular}{|c|c|c|c|c|}
\hline Atom & $\mathbf{x}$ & $\mathbf{y}$ & $\mathbf{z}$ & $U_{e q}$ \\
\hline$\overline{\mathrm{C} 162}$ & $2840(11)$ & $5215(6)$ & $8407(6)$ & $38(7)$ \\
\hline $\mathrm{C} 163$ & $2808(11)$ & $5084(6)$ & $8741(6)$ & $43(7)$ \\
\hline C164 & $2706(12)$ & $4717(7)$ & $8764(7)$ & $59(8)$ \\
\hline $\mathrm{C} 165$ & 2633(13) & $4512(7)$ & $8490(7)$ & $71(9)$ \\
\hline C166 & $2643(14)$ & $4633(8)$ & $8166(7)$ & $80(9)$ \\
\hline $\mathrm{C} 167$ & $2739(13)$ & $4972(7)$ & $8144(6)$ & $58(8)$ \\
\hline $\mathrm{C} 168$ & $2896(12)$ & $5066(6)$ & $7495(5)$ & $58(8)$ \\
\hline C169 & $2886(11)$ & $5524(6)$ & $7930(6)$ & $37(7)$ \\
\hline C170 & 2942(11) & $5818(6)$ & $7744(6)$ & $31(7)$ \\
\hline $\mathrm{C} 171$ & $2880(11)$ & $5863(6)$ & $7388(6)$ & $49(7)$ \\
\hline C172 & 2917(13) & $6194(7)$ & $7259(7)$ & $78(9)$ \\
\hline $\mathrm{C} 173$ & $3024(11)$ & $6486(6)$ & $7455(6)$ & $37(7)$ \\
\hline $\mathrm{C} 174$ & $3127(11)$ & $6410(6)$ & $7795(6)$ & $43(7)$ \\
\hline $\mathrm{C} 175$ & $3043(11)$ & $6840(6)$ & $7329(6)$ & $38(7)$ \\
\hline $\mathrm{C} 176$ & $3089(14)$ & $6888(8)$ & $6979(8)$ & $91(10)$ \\
\hline C177 & $3184(15)$ & $7232(8)$ & $6852(8)$ & $94(10)$ \\
\hline $\mathrm{C} 178$ & $3124(13)$ & $7513(7)$ & $7053(7)$ & $57(8)$ \\
\hline C179 & $3048(13)$ & $7470(7)$ & $7398(7)$ & $68(9)$ \\
\hline C180 & $3026(15)$ & $7125(8)$ & $7518(8)$ & $98(10)$ \\
\hline C181 & $3710(11)$ & $9165(6)$ & $6038(6)$ & $33(7)$ \\
\hline C182 & $4059(11)$ & $9304(6)$ & $5728(5)$ & $36(7)$ \\
\hline C183 & 3991(11) & $9626(6)$ & $5673(5)$ & $29(6)$ \\
\hline C184 & $3633(12)$ & $9843(6)$ & $5881(6)$ & $46(7)$ \\
\hline C185 & $3308(13)$ & $9717(7)$ & $6157(6)$ & $64(8)$ \\
\hline C186 & $3346(12)$ & $9370(6)$ & $6220(6)$ & $38(7)$ \\
\hline C187 & $2698(15)$ & $9261(8)$ & $6788(6)$ & $106(11)$ \\
\hline C188 & $3265(12)$ & $8848(6)$ & $6423(6)$ & $43(7)$ \\
\hline C189 & $3201(12)$ & $8520(6)$ & $6624(6)$ & $42(7)$ \\
\hline C190 & $2862(12)$ & $8467(6)$ & $6923(6)$ & $49(7)$ \\
\hline C191 & $2849(12)$ & $8156(6)$ & $7075(6)$ & $58(8)$ \\
\hline C192 & $3174(12)$ & $7860(6)$ & $6915(6)$ & $46(7)$ \\
\hline C193 & $3512(10)$ & $7930(6)$ & $6606(5)$ & $30(6)$ \\
\hline $\mathrm{P} 1$ & $796(5)$ & $5056(2)$ & $7620(2)$ & 81(3) \\
\hline F5 & $1348(6)$ & $5353(3)$ & $7583(4)$ & $107(6)$ \\
\hline $\mathrm{F} 2$ & $1418(6)$ & $4781(3)$ & $7548(3)$ & $100(6)$ \\
\hline F1 & 204(6) & $5318(3)$ & $7673(3)$ & $98(6)$ \\
\hline $\mathrm{F} 4$ & $657(7)$ & $5068(3)$ & $7218(3)$ & $106(6)$ \\
\hline F3 & $973(8)$ & $5026(3)$ & $8003(3)$ & $116(7)$ \\
\hline F6 & $247(6)$ & $4756(3)$ & $7635(3)$ & $101(6)$ \\
\hline $\mathrm{P} 2$ & $8047(5)$ & $5456(3)$ & $8469(2)$ & $89(3)$ \\
\hline F7 & $7902(8)$ & $5751(5)$ & $8220(4)$ & $122(5)$ \\
\hline F8 & $8194(8)$ & $5192(5)$ & $8764(4)$ & $123(5)$ \\
\hline F9 & $7278(8)$ & $5515(4)$ & $8624(4)$ & 98(4) \\
\hline F10 & $8831(7)$ & $5411(4)$ & $8305(4)$ & $96(4)$ \\
\hline F11 & $8382(9)$ & $5697(6)$ & $8754(5)$ & $144(5)$ \\
\hline F12 & $7758(8)$ & $5172(5)$ & $8243(4)$ & $112(5)$ \\
\hline P3 & $1029(5)$ & $8717(3)$ & $7409(2)$ & $116(3)$ \\
\hline F13 & $657(9)$ & $9075(4)$ & $7355(4)$ & $163(6)$ \\
\hline F14 & $1179(7)$ & $8701(4)$ & $7007(3)$ & $124(5)$ \\
\hline F15 & $863(7)$ & $8730(4)$ & 7793(3) & $120(5)$ \\
\hline F16 & $279(7)$ & $8550(4)$ & $7321(4)$ & $155(6)$ \\
\hline F17 & $1746(7)$ & $8893(5)$ & $7471(4)$ & $161(6)$ \\
\hline F18 & $1363(9)$ & $8358(4)$ & $7455(4)$ & $177(6)$ \\
\hline P4 & 2619(9) & $7420(5)$ & $9596(4)$ & $178(5)$ \\
\hline F19 & $2720(13)$ & $7825(8)$ & $9662(6)$ & 199(7) \\
\hline F20 & $2444(13)$ & $6997(7)$ & $9425(6)$ & $204(7)$ \\
\hline F21 & $2368(11)$ & $7600(6)$ & $9229(5)$ & $175(7)$ \\
\hline F22 & $2862(13)$ & $7261(7)$ & $9899(6)$ & $205(7)$ \\
\hline F23 & $1847(14)$ & $7409(7)$ & $9737(5)$ & $192(7)$ \\
\hline F24 & $3322(14)$ & $7366(7)$ & $9488(6)$ & $185(7)$ \\
\hline P5 & $9745(5)$ & $7350(3)$ & $5337(2)$ & 74(3) \\
\hline F25 & $9704(11)$ & $7726(6)$ & $5223(5)$ & $137(7)$ \\
\hline F26 & $9819(9)$ & $6979(5)$ & $5464(4)$ & $104(6)$ \\
\hline F27 & $9013(12)$ & $7353(6)$ & $5491(5)$ & $162(9)$ \\
\hline
\end{tabular}




\begin{tabular}{|c|c|c|c|c|}
\hline Atom & $\mathbf{x}$ & $\mathbf{y}$ & $\mathbf{z}$ & $\boldsymbol{U}_{e q}$ \\
\hline$\overline{\text { F28 }}$ & $10468(10)$ & $7334(6)$ & $5167(5)$ & $143(8)$ \\
\hline F29 & 9361(11) & $7227(5)$ & 4993(4) & $145(8)$ \\
\hline F30 & 10111(11) & $7458(5)$ & $5697(5)$ & $148(8)$ \\
\hline P6 & $6189(5)$ & $9584(2)$ & $5274(2)$ & $91(3)$ \\
\hline F31 & $6407(10)$ & $9814(4)$ & 4979(4) & 196 \\
\hline F32 & $5963(9)$ & $9338(4)$ & $5579(4)$ & 172 \\
\hline F33 & $5414(8)$ & $9709(5)$ & $5242(5)$ & 304 \\
\hline F34 & $6962(7)$ & $9439(4)$ & $5326(4)$ & 131 \\
\hline F35 & $6378(8)$ & $9873(3)$ & $5536(3)$ & 121 \\
\hline F36 & $6027(9)$ & $9289(4)$ & $5008(4)$ & 163 \\
\hline P7 & $8485(7)$ & $8000(3)$ & $6627(3)$ & $177(5)$ \\
\hline F37 & $7786(10)$ & $8067(6)$ & $6425(6)$ & 300 \\
\hline F38 & $9214(11)$ & $7934(7)$ & $6848(6)$ & 370 \\
\hline F39 & $8822(12)$ & $7830(6)$ & $6317(5)$ & 260 \\
\hline F40 & $8168(14)$ & $8155(6)$ & $6959(5)$ & 336 \\
\hline F41 & $8205(10)$ & $7638(4)$ & $6744(5)$ & 177 \\
\hline F42 & $8769(12)$ & $8355(5)$ & $6522(6)$ & 242 \\
\hline P8 & $5189(7)$ & $6753(4)$ & $7756(3)$ & $77(8)$ \\
\hline F43 & $5002(14)$ & $6971(6)$ & $7436(5)$ & 165 \\
\hline F44 & $5416(11)$ & $6534(5)$ & $8091(5)$ & 85 \\
\hline F45 & $4757(11)$ & $6449(5)$ & $7614(5)$ & 106 \\
\hline F46 & $5657(12)$ & $7057(5)$ & $7909(6)$ & 157 \\
\hline F47 & $5878(11)$ & $6619(6)$ & $7578(6)$ & 146 \\
\hline F48 & $4537(10)$ & $6897(5)$ & $7937(5)$ & 88 \\
\hline P8A & $6862(9)$ & $6723(4)$ & $7147(4)$ & $121(10)$ \\
\hline F43A & $6447(14)$ & $7038(6)$ & $7015(7)$ & 176 \\
\hline F44A & $7312(14)$ & $6405(5)$ & $7302(6)$ & 133 \\
\hline $\mathrm{F} 45 \mathrm{~A}$ & $6568(13)$ & $6494(6)$ & $6861(6)$ & 110 \\
\hline F46A & $7161(14)$ & $6942(6)$ & $7464(5)$ & 133 \\
\hline F47A & $6222(13)$ & $6621(8)$ & $7379(7)$ & 277 \\
\hline F48A & $7506(12)$ & $6830(6)$ & $6938(6)$ & 134 \\
\hline P9 & $3537(9)$ & $8055(4)$ & $8242(4)$ & 279(9) \\
\hline F49 & $3251(12)$ & $8151(6)$ & $7885(5)$ & 253 \\
\hline F50 & $3853(12)$ & $7967(6)$ & $8623(5)$ & 251 \\
\hline F51 & 2981(10) & $7774(5)$ & $8279(5)$ & 217 \\
\hline F52 & $4142(12)$ & $8333(6)$ & $8218(7)$ & 310 \\
\hline F53 & $4070(11)$ & $7787(6)$ & $8095(6)$ & 284 \\
\hline F54 & $3040(12)$ & $8327(5)$ & $8401(6)$ & 258 \\
\hline $\mathrm{P} 10$ & $8028(8)$ & $4428(4)$ & $5215(4)$ & 274(9) \\
\hline F55 & $7942(11)$ & $4210(5)$ & $4895(5)$ & 225 \\
\hline F56 & $8130(11)$ & $4674(5)$ & $5549(4)$ & 208 \\
\hline F57 & $7802(12)$ & $4140(5)$ & $5450(5)$ & 256 \\
\hline F58 & $8280(11)$ & $4739(5)$ & $4990(5)$ & 241 \\
\hline F59 & $8832(9)$ & $4323(5)$ & $5266(5)$ & 227 \\
\hline F60 & 7249(9) & $4562(5)$ & $5172(5)$ & 220 \\
\hline P11 & $13406(8)$ & $6526(4)$ & $5996(4)$ & $256(8)$ \\
\hline F61 & $12677(9)$ & $6674(5)$ & $6040(5)$ & 243 \\
\hline F62 & $14204(8)$ & $6368(5)$ & $5944(5)$ & 192 \\
\hline F63 & $13404(10)$ & $6336(5)$ & $6342(4)$ & 190 \\
\hline F64 & $13458(10)$ & $6699(5)$ & $5634(4)$ & 192 \\
\hline F65 & $13099(10)$ & $6200(4)$ & $5805(5)$ & 199 \\
\hline F66 & $13762(11)$ & $6846(5)$ & $6164(5)$ & 249 \\
\hline $\mathrm{P} 12$ & $6749(9)$ & $7734(4)$ & 7991(4) & $377(13)$ \\
\hline F67 & $7242(10)$ & $7790(5)$ & $7678(4)$ & 222 \\
\hline F68 & $6339(9)$ & $8094(4)$ & $7882(5)$ & 174 \\
\hline F69 & $7101(9)$ & $7402(4)$ & $8081(4)$ & 163 \\
\hline F70 & $6195(10)$ & $7552(5)$ & $7731(5)$ & 222 \\
\hline F71 & $7258(10)$ & $7944(5)$ & $8218(5)$ & 212 \\
\hline F72 & $6214(10)$ & $7698(5)$ & $8280(5)$ & 238 \\
\hline
\end{tabular}

Table S3: Anisotropic Displacement Parameters $\left(\times 10^{4}\right)$ PL15004. The anisotropic displacement factor exponent takes the form: $-2 \pi^{2}\left[a^{* 2} \times U_{11}+\ldots 2 h k a * \times b^{*} \times U_{12}\right]$ 


\begin{tabular}{|c|c|c|c|c|c|c|}
\hline Atom & $U_{11}$ & $U_{22}$ & $\boldsymbol{U}_{33}$ & $\boldsymbol{U}_{23}$ & $U_{13}$ & $U_{12}$ \\
\hline$\overline{\mathrm{Co} 1}$ & $33(3)$ & $42(3)$ & $14(2)$ & $1(2)$ & $-0.2(19)$ & $-8(2)$ \\
\hline $\mathrm{Co} 2$ & $45(3)$ & $70(3)$ & $17(2)$ & $-10(2)$ & $2(2)$ & $14(2)$ \\
\hline Co3 & $31(3)$ & $53(3)$ & $16(2)$ & $6(2)$ & 3.1(19) & $-5(2)$ \\
\hline $\mathrm{Co} 4$ & $34(3)$ & $62(3)$ & $14(2)$ & $11(2)$ & $-3.7(19)$ & $-3(2)$ \\
\hline P1 & $85(8)$ & $57(7)$ & $98(9)$ & $-8(6)$ & $-21(6)$ & $-6(7)$ \\
\hline F5 & $62(12)$ & $99(14)$ & $158(17)$ & $-22(12)$ & $-37(11)$ & $-9(11)$ \\
\hline $\mathrm{F} 2$ & $74(12)$ & $71(12)$ & $151(16)$ & $-26(11)$ & $-36(11)$ & $12(10)$ \\
\hline F1 & $95(13)$ & $85(13)$ & $112(14)$ & $-31(10)$ & $-20(10)$ & $44(11)$ \\
\hline $\mathrm{F} 4$ & $169(17)$ & $103(14)$ & $45(11)$ & 3(9) & $-20(11)$ & $15(12)$ \\
\hline F3 & $180(18)$ & $112(15)$ & 54(12) & $-14(10)$ & $-43(12)$ & $36(13)$ \\
\hline F6 & $92(14)$ & 84(13) & $126(15)$ & $-19(11)$ & $10(11)$ & $33(12)$ \\
\hline $\mathrm{P} 2$ & $57(6)$ & $136(8)$ & $74(6)$ & $19(5)$ & $8(5)$ & $-32(5)$ \\
\hline F7 & $80(9)$ & $167(10)$ & $121(10)$ & $45(7)$ & $24(7)$ & $-24(8)$ \\
\hline F8 & $75(9)$ & $192(11)$ & $104(9)$ & $45(7)$ & $17(7)$ & $-24(8)$ \\
\hline F9 & $68(7)$ & 151(11) & $78(9)$ & $11(8)$ & $16(6)$ & $-32(7)$ \\
\hline F10 & $59(7)$ & $148(11)$ & $80(9)$ & $12(8)$ & $3(6)$ & $-37(7)$ \\
\hline F11 & 107(10) & $211(12)$ & 115(9) & $-12(8)$ & $16(7)$ & $-46(9)$ \\
\hline F12 & $60(9)$ & $164(10)$ & 112(9) & $-1(7)$ & $19(7)$ & $-35(7)$ \\
\hline P3 & $111(8)$ & $139(8)$ & $103(6)$ & $-27(7)$ & $55(6)$ & 1(6) \\
\hline F13 & 199(13) & 177(10) & $114(11)$ & $-31(8)$ & $48(10)$ & $29(9)$ \\
\hline F14 & $108(11)$ & $157(13)$ & $111(8)$ & $-34(8)$ & $49(7)$ & $-10(9)$ \\
\hline F15 & $116(11)$ & $141(12)$ & $106(7)$ & $-22(8)$ & $52(7)$ & $14(9)$ \\
\hline F16 & 137(9) & $207(13)$ & $124(11)$ & $-24(10)$ & $56(8)$ & $-19(8)$ \\
\hline F17 & $145(10)$ & $230(14)$ & 111(11) & $-34(10)$ & $55(8)$ & $-31(9)$ \\
\hline F18 & $198(13)$ & $184(10)$ & $152(12)$ & $-26(9)$ & $53(10)$ & $32(9)$ \\
\hline P4 & $166(11)$ & $242(12)$ & $125(9)$ & $-10(9)$ & $-23(8)$ & $90(11)$ \\
\hline F19 & 197(16) & $259(13)$ & $140(13)$ & $-19(10)$ & $-26(11)$ & $76(11)$ \\
\hline F20 & $205(16)$ & $250(13)$ & $155(14)$ & $-9(10)$ & $-27(12)$ & $82(11)$ \\
\hline F21 & $160(14)$ & $241(15)$ & $122(10)$ & $-15(9)$ & $-10(9)$ & $97(12)$ \\
\hline F22 & $192(14)$ & $278(16)$ & $141(11)$ & 1(10) & $-26(9)$ & $97(13)$ \\
\hline F23 & $180(12)$ & $257(17)$ & $136(13)$ & $-14(11)$ & $-15(9)$ & $81(11)$ \\
\hline F24 & $169(11)$ & $242(16)$ & $142(13)$ & $-21(11)$ & $-20(9)$ & $86(11)$ \\
\hline P5 & $74(9)$ & 89(11) & $59(8)$ & $7(7)$ & $0(8)$ & $-20(6)$ \\
\hline F25 & $180(20)$ & $104(18)$ & $133(17)$ & $12(14)$ & $28(14)$ & $-3(15)$ \\
\hline F26 & $135(17)$ & $102(16)$ & $75(12)$ & $15(11)$ & $2(10)$ & $-7(12)$ \\
\hline F27 & $128(19)$ & $190(20)$ & $170(20)$ & $97(16)$ & $74(16)$ & $22(16)$ \\
\hline F28 & $73(15)$ & $240(30)$ & $121(16)$ & $-11(15)$ & 17(13) & 1(14) \\
\hline F29 & $210(20)$ & $160(19)$ & $64(13)$ & $26(12)$ & $-45(14)$ & $-82(16)$ \\
\hline F30 & $156(19)$ & $190(20)$ & $93(16)$ & $19(14)$ & $-36(14)$ & $-77(16)$ \\
\hline
\end{tabular}

Table S4: Bond Lengths in $\AA$ for PL15004.

\begin{tabular}{lll}
\hline Atom & Atom & Length $/ \AA$ \\
\hline Co1 & N1 & $1.985(17)$ \\
Co1 & N2 & $2.021(17)$ \\
Co1 & N7 & $1.951(18)$ \\
Co1 & N2 & $1.923(16)$ \\
Co1 & N13 & $1.932(16)$ \\
Co1 & N14 & $1.912(18)$ \\
Co2 & N4 & $1.967(16)$ \\
Co2 & N5 & $1.903(19)$ \\
Co2 & N19 & $1.947(19)$ \\
Co2 & N20 & $1.974(14)$ \\
Co2 & N25 & $1.998(18)$ \\
Co2 & N26 & $1.920(19)$ \\
Co3 & N11 & $1.940(17)$ \\
Co3 & N23 & $1.902(16)$ \\
Co3 & N31 & $1.978(17)$ \\
Co3 & N32 & $1.893(17)$ \\
Co3 & N10 & $1.973(11)$ \\
Co3 & N22 & $1.946(11)$ \\
Co4 & N16 & $1.976(16)$ \\
Co4 & N17 & $1.975(18)$
\end{tabular}

\begin{tabular}{|c|c|c|}
\hline Atom & Atom & Length/Å \\
\hline $\mathrm{Co} 4$ & N28 & $1.935(16)$ \\
\hline $\mathrm{Co} 4$ & N29 & $1.925(13)$ \\
\hline $\mathrm{Co} 4$ & N34 & $1.984(16)$ \\
\hline $\mathrm{Co} 4$ & N35 & $1.955(18)$ \\
\hline N1 & C9 & $1.34(2)$ \\
\hline N1 & C13 & $1.33(2)$ \\
\hline N2 & $\mathrm{C} 1$ & $1.30(2)$ \\
\hline N2 & $\mathrm{C} 8$ & $1.31(2)$ \\
\hline N3 & C6 & $1.39(2)$ \\
\hline N3 & $\mathrm{C} 7$ & $1.435(14)$ \\
\hline N3 & $\mathrm{C} 8$ & $1.32(2)$ \\
\hline N4 & $\mathrm{C} 28$ & $1.30(2)$ \\
\hline N4 & $\mathrm{C} 32$ & $1.35(2)$ \\
\hline N5 & $\mathrm{C} 20$ & $1.45(3)$ \\
\hline N5 & $\mathrm{C} 27$ & $1.33(2)$ \\
\hline N6 & $\mathrm{C} 25$ & $1.55(3)$ \\
\hline N6 & $\mathrm{C} 26$ & $1.442(14)$ \\
\hline N6 & $\mathrm{C} 27$ & $1.33(2)$ \\
\hline N7 & C41 & $1.37(2)$ \\
\hline N7 & $\mathrm{C} 45$ & $1.33(2)$ \\
\hline
\end{tabular}




\begin{tabular}{|c|c|c|}
\hline$\overline{\text { Atom }}$ & Atom & Length/Å \\
\hline N8 & C33 & $1.35(2)$ \\
\hline N8 & $\mathrm{C} 40$ & $1.33(2)$ \\
\hline N9 & C38 & $1.33(2)$ \\
\hline N9 & C39 & $1.439(14)$ \\
\hline N9 & $\mathrm{C} 40$ & $1.30(2)$ \\
\hline N11 & C52 & $1.45(2)$ \\
\hline N11 & C59 & $1.31(2)$ \\
\hline N12 & C57 & $1.37(2)$ \\
\hline N12 & C58 & $1.445(15)$ \\
\hline $\mathrm{N} 12$ & C59 & $1.40(2)$ \\
\hline N13 & $\mathrm{C} 73$ & $1.35(2)$ \\
\hline N13 & C77 & $1.38(2)$ \\
\hline N14 & C65 & $1.42(2)$ \\
\hline $\mathrm{N} 14$ & C72 & $1.23(2)$ \\
\hline N15 & $\mathrm{C} 70$ & $1.44(3)$ \\
\hline N15 & C71 & $1.444(15)$ \\
\hline N15 & $\mathrm{C} 72$ & $1.31(2)$ \\
\hline N16 & C92 & $1.30(2)$ \\
\hline N16 & C96 & $1.38(2)$ \\
\hline N17 & $\mathrm{C} 84$ & $1.38(2)$ \\
\hline N17 & C91 & $1.32(3)$ \\
\hline N18 & C89 & $1.37(2)$ \\
\hline N18 & C90 & $1.442(14)$ \\
\hline N18 & C91 & $1.30(3)$ \\
\hline N19 & C105 & $1.40(3)$ \\
\hline N19 & C109 & $1.36(2)$ \\
\hline $\mathrm{N} 21$ & C104 & 1.4200 \\
\hline $\mathrm{N} 21$ & C102 & 1.4200 \\
\hline N21 & C103 & $1.428(16)$ \\
\hline C104 & N20 & 1.4200 \\
\hline C104 & C105 & $1.48(3)$ \\
\hline N20 & C97 & 1.4200 \\
\hline C97 & C102 & 1.4200 \\
\hline C97 & C98 & $1.32(3)$ \\
\hline $\mathrm{C} 102$ & C101 & $1.46(4)$ \\
\hline N23 & C116 & $1.41(2)$ \\
\hline $\mathrm{N} 23$ & C123 & $1.31(2)$ \\
\hline $\mathrm{N} 24$ & C121 & $1.35(2)$ \\
\hline N24 & C122 & $1.473(15)$ \\
\hline N24 & C123 & $1.38(2)$ \\
\hline $\mathrm{N} 25$ & C138 & $1.30(2)$ \\
\hline $\mathrm{N} 25$ & C142 & $1.31(2)$ \\
\hline $\mathrm{N} 27$ & C137 & $1.39(2)$ \\
\hline $\mathrm{N} 27$ & C135 & $1.380(19)$ \\
\hline $\mathrm{N} 27$ & C136 & $1.429(14)$ \\
\hline C137 & N26 & $1.34(2)$ \\
\hline C137 & C138 & $1.49(3)$ \\
\hline N26 & C130 & $1.334(19)$ \\
\hline C135 & C134 & 1.3900 \\
\hline C135 & C130 & 1.3900 \\
\hline C134 & C133 & 1.3900 \\
\hline C133 & C132 & 1.3900 \\
\hline C132 & C131 & 1.3900 \\
\hline C131 & C130 & 1.3900 \\
\hline N28 & C157 & $1.38(3)$ \\
\hline N28 & C161 & $1.34(2)$ \\
\hline N29 & C156 & 1.4200 \\
\hline N29 & C149 & 1.4200 \\
\hline C156 & N30 & 1.4200 \\
\hline C156 & C157 & $1.39(3)$ \\
\hline N30 & C154 & 1.4200 \\
\hline N30 & C155 & $1.446(15)$ \\
\hline C154 & C149 & 1.4200 \\
\hline C154 & C153 & $1.43(4)$ \\
\hline
\end{tabular}

\begin{tabular}{|c|c|c|}
\hline Atom & Atom & Length/Å \\
\hline$\overline{\mathrm{C} 149}$ & $\mathrm{C} 150$ & $1.38(3)$ \\
\hline $\mathrm{C} 150$ & $\mathrm{C} 151$ & $1.46(4)$ \\
\hline C151 & C152 & $1.39(4)$ \\
\hline C152 & C153 & $1.44(4)$ \\
\hline N31 & $\mathrm{C} 170$ & $1.39(2)$ \\
\hline N31 & C174 & $1.32(2)$ \\
\hline N32 & $\mathrm{C} 162$ & $1.38(2)$ \\
\hline N32 & C169 & $1.39(2)$ \\
\hline N33 & $\mathrm{C} 167$ & $1.49(3)$ \\
\hline N33 & $\mathrm{C} 168$ & $1.414(14)$ \\
\hline N33 & C169 & $1.34(2)$ \\
\hline N34 & C189 & $1.36(2)$ \\
\hline N34 & C193 & $1.33(2)$ \\
\hline N35 & C181 & $1.39(2)$ \\
\hline N35 & C188 & $1.35(2)$ \\
\hline N36 & C186 & $1.41(2)$ \\
\hline N36 & C187 & $1.446(15)$ \\
\hline N36 & C188 & $1.30(2)$ \\
\hline $\mathrm{C} 1$ & $\mathrm{C} 2$ & $1.38(3)$ \\
\hline $\mathrm{C} 1$ & C6 & $1.35(2)$ \\
\hline $\mathrm{C} 2$ & $\mathrm{C} 3$ & $1.40(3)$ \\
\hline $\mathrm{C} 3$ & $\mathrm{C} 4$ & $1.50(3)$ \\
\hline $\mathrm{C} 4$ & $\mathrm{C} 5$ & $1.34(3)$ \\
\hline $\mathrm{C} 5$ & C6 & $1.30(3)$ \\
\hline $\mathrm{C} 8$ & C9 & $1.42(2)$ \\
\hline C9 & $\mathrm{C} 10$ & $1.42(3)$ \\
\hline $\mathrm{C} 10$ & $\mathrm{C} 11$ & $1.38(3)$ \\
\hline $\mathrm{C} 11$ & $\mathrm{C} 12$ & $1.34(3)$ \\
\hline $\mathrm{C} 12$ & $\mathrm{C} 13$ & $1.33(2)$ \\
\hline $\mathrm{C} 12$ & $\mathrm{C} 14$ & $1.46(3)$ \\
\hline $\mathrm{C} 14$ & $\mathrm{C} 15$ & $1.53(3)$ \\
\hline $\mathrm{C} 14$ & C19 & $1.41(3)$ \\
\hline $\mathrm{C} 15$ & $\mathrm{C} 16$ & $1.38(3)$ \\
\hline $\mathrm{C} 16$ & $\mathrm{C} 17$ & $1.43(3)$ \\
\hline $\mathrm{C} 17$ & $\mathrm{C} 18$ & $1.45(3)$ \\
\hline $\mathrm{C} 17$ & C31 & $1.46(3)$ \\
\hline $\mathrm{C} 18$ & C19 & $1.33(3)$ \\
\hline $\mathrm{C} 20$ & $\mathrm{C} 21$ & $1.41(3)$ \\
\hline $\mathrm{C} 20$ & $\mathrm{C} 25$ & $1.35(3)$ \\
\hline $\mathrm{C} 21$ & $\mathrm{C} 22$ & $1.40(3)$ \\
\hline $\mathrm{C} 22$ & $\mathrm{C} 23$ & $1.38(3)$ \\
\hline $\mathrm{C} 23$ & $\mathrm{C} 24$ & $1.31(3)$ \\
\hline $\mathrm{C} 24$ & $\mathrm{C} 25$ & $1.32(3)$ \\
\hline $\mathrm{C} 27$ & $\mathrm{C} 28$ & $1.49(3)$ \\
\hline $\mathrm{C} 28$ & $\mathrm{C} 29$ & $1.47(3)$ \\
\hline $\mathrm{C} 29$ & $\mathrm{C} 30$ & $1.32(3)$ \\
\hline $\mathrm{C} 30$ & C31 & $1.42(3)$ \\
\hline C31 & $\mathrm{C} 32$ & $1.39(2)$ \\
\hline C33 & C34 & $1.49(3)$ \\
\hline C33 & C38 & $1.43(3)$ \\
\hline C34 & C35 & $1.32(3)$ \\
\hline $\mathrm{C} 35$ & $\mathrm{C} 36$ & $1.38(3)$ \\
\hline $\mathrm{C} 36$ & $\mathrm{C} 37$ & $1.54(4)$ \\
\hline $\mathrm{C} 37$ & C38 & $1.46(3)$ \\
\hline $\mathrm{C} 40$ & $\mathrm{C} 41$ & $1.44(2)$ \\
\hline $\mathrm{C} 41$ & $\mathrm{C} 42$ & $1.33(3)$ \\
\hline $\mathrm{C} 42$ & $\mathrm{C} 43$ & $1.45(3)$ \\
\hline $\mathrm{C} 43$ & $\mathrm{C} 44$ & $1.32(3)$ \\
\hline $\mathrm{C} 44$ & $\mathrm{C} 45$ & $1.37(2)$ \\
\hline $\mathrm{C} 44$ & $\mathrm{C} 46$ & $1.49(3)$ \\
\hline $\mathrm{C} 46$ & $\mathrm{C} 47$ & $1.37(3)$ \\
\hline $\mathrm{C} 46$ & C51 & $1.38(3)$ \\
\hline $\mathrm{C} 47$ & $\mathrm{C} 48$ & $1.35(3)$ \\
\hline $\mathrm{C} 48$ & C49 & $1.46(3)$ \\
\hline
\end{tabular}




\begin{tabular}{|c|c|c|}
\hline$\overline{\text { Atom }}$ & Atom & Length/Å \\
\hline$\overline{\mathrm{C} 49}$ & $\mathrm{C} 50$ & $1.40(3)$ \\
\hline C49 & C64 & $1.38(2)$ \\
\hline $\mathrm{C} 50$ & C51 & $1.42(3)$ \\
\hline C52 & C53 & $1.40(3)$ \\
\hline C52 & $\mathrm{C} 57$ & $1.31(3)$ \\
\hline C53 & C54 & $1.35(3)$ \\
\hline C54 & C55 & $1.39(3)$ \\
\hline C55 & C56 & $1.33(3)$ \\
\hline C56 & C57 & $1.51(3)$ \\
\hline C59 & $\mathrm{C} 60$ & $1.42(2)$ \\
\hline C61 & C62 & 1.3900 \\
\hline C61 & C60 & 1.3900 \\
\hline C62 & C64 & 1.3900 \\
\hline C64 & C63 & 1.3900 \\
\hline C63 & N10 & 1.3900 \\
\hline N10 & C60 & 1.3900 \\
\hline C65 & C66 & $1.40(3)$ \\
\hline C65 & C70 & $1.28(3)$ \\
\hline C66 & C67 & $1.38(3)$ \\
\hline C67 & C68 & $1.32(3)$ \\
\hline C68 & C69 & $1.28(3)$ \\
\hline C69 & C70 & $1.54(3)$ \\
\hline $\mathrm{C} 72$ & C73 & $1.50(3)$ \\
\hline C73 & C74 & $1.38(3)$ \\
\hline C74 & C75 & $1.44(3)$ \\
\hline C75 & C76 & $1.36(3)$ \\
\hline C76 & C77 & $1.31(3)$ \\
\hline C76 & $\mathrm{C} 78$ & $1.52(3)$ \\
\hline $\mathrm{C} 78$ & C79 & 1.3900 \\
\hline $\mathrm{C} 78$ & $\mathrm{C} 83$ & 1.3900 \\
\hline C79 & $\mathrm{C} 80$ & 1.3900 \\
\hline $\mathrm{C} 80$ & C81 & 1.3900 \\
\hline C81 & $\mathrm{C} 82$ & 1.3900 \\
\hline C81 & C95 & $1.49(2)$ \\
\hline $\mathrm{C} 82$ & $\mathrm{C} 83$ & 1.3900 \\
\hline C84 & $\mathrm{C} 85$ & $1.36(3)$ \\
\hline C84 & C89 & $1.35(3)$ \\
\hline $\mathrm{C} 85$ & $\mathrm{C} 86$ & $1.43(3)$ \\
\hline C86 & C87 & $1.36(3)$ \\
\hline C87 & $\mathrm{C} 88$ & $1.37(3)$ \\
\hline C88 & C89 & $1.45(3)$ \\
\hline C91 & $\mathrm{C} 92$ & $1.54(3)$ \\
\hline C92 & C93 & $1.28(3)$ \\
\hline C93 & C94 & $1.48(3)$ \\
\hline C94 & C95 & $1.43(3)$ \\
\hline C95 & C96 & $1.38(3)$ \\
\hline C101 & $\mathrm{C} 100$ & $1.36(5)$ \\
\hline $\mathrm{C} 100$ & C99 & $1.34(4)$ \\
\hline C99 & C98 & $1.43(3)$ \\
\hline C105 & C106 & $1.27(4)$ \\
\hline C106 & $\mathrm{C} 107$ & $1.53(4)$ \\
\hline C107 & C108 & $1.35(3)$ \\
\hline C108 & C109 & $1.30(3)$ \\
\hline C108 & C110 & $1.56(3)$ \\
\hline C110 & C111 & 1.3900 \\
\hline $\mathrm{C} 110$ & C115 & 1.3900 \\
\hline C111 & C112 & 1.3900 \\
\hline C112 & C113 & 1.3900 \\
\hline C113 & C114 & 1.3900 \\
\hline C113 & $\mathrm{C} 127$ & $1.511(15)$ \\
\hline C114 & C115 & 1.3900 \\
\hline C116 & C117 & $1.39(3)$ \\
\hline C116 & C121 & $1.33(2)$ \\
\hline $\mathrm{C} 117$ & C118 & $1.53(3)$ \\
\hline
\end{tabular}

\begin{tabular}{|c|c|c|}
\hline Atom & Atom & Length/Å \\
\hline$\overline{C 118}$ & C119 & $1.33(3)$ \\
\hline C119 & $\mathrm{C} 120$ & $1.37(3)$ \\
\hline C120 & C121 & $1.46(3)$ \\
\hline C123 & C124 & $1.42(2)$ \\
\hline C124 & $\mathrm{C} 125$ & 1.3900 \\
\hline $\mathrm{C} 124$ & N22 & 1.3900 \\
\hline $\mathrm{C} 125$ & $\mathrm{C} 126$ & 1.3900 \\
\hline C126 & $\mathrm{C} 127$ & 1.3900 \\
\hline C127 & C128 & 1.3900 \\
\hline C128 & N22 & 1.3900 \\
\hline C138 & C139 & $1.32(2)$ \\
\hline C139 & C140 & $1.33(3)$ \\
\hline $\mathrm{C} 140$ & C141 & $1.36(3)$ \\
\hline C141 & C142 & $1.37(3)$ \\
\hline C141 & C143 & $1.52(2)$ \\
\hline C143 & C148 & 1.3900 \\
\hline C143 & C144 & 1.3900 \\
\hline C148 & $\mathrm{C} 147$ & 1.3900 \\
\hline $\mathrm{C} 147$ & C146 & 1.3900 \\
\hline C146 & C145 & 1.3900 \\
\hline C146 & $\mathrm{C} 160$ & $1.44(2)$ \\
\hline C145 & C144 & 1.3900 \\
\hline C157 & C158 & $1.25(3)$ \\
\hline C158 & C159 & $1.41(4)$ \\
\hline C159 & $\mathrm{C} 160$ & $1.48(4)$ \\
\hline C160 & $\mathrm{C} 161$ & $1.39(3)$ \\
\hline C162 & $\mathrm{C} 163$ & $1.39(3)$ \\
\hline C162 & $\mathrm{C} 167$ & $1.40(3)$ \\
\hline C163 & C164 & $1.44(3)$ \\
\hline C164 & $\mathrm{C} 165$ & $1.33(3)$ \\
\hline C165 & C166 & $1.34(3)$ \\
\hline C166 & C167 & $1.33(3)$ \\
\hline C169 & $\mathrm{C} 170$ & $1.36(3)$ \\
\hline $\mathrm{C} 170$ & $\mathrm{C} 171$ & $1.39(3)$ \\
\hline $\mathrm{C} 171$ & $\mathrm{C} 172$ & $1.38(3)$ \\
\hline C172 & C173 & $1.38(3)$ \\
\hline C173 & C174 & $1.36(3)$ \\
\hline C173 & $\mathrm{C} 175$ & $1.46(3)$ \\
\hline C175 & C176 & $1.38(3)$ \\
\hline C175 & C180 & $1.33(3)$ \\
\hline C176 & $\mathrm{C} 177$ & $1.43(3)$ \\
\hline C177 & $\mathrm{C} 178$ & $1.35(3)$ \\
\hline C178 & C179 & $1.36(3)$ \\
\hline C178 & C192 & $1.45(3)$ \\
\hline C179 & C180 & $1.42(3)$ \\
\hline C181 & C182 & $1.49(3)$ \\
\hline C181 & C186 & $1.28(2)$ \\
\hline C182 & C183 & $1.27(2)$ \\
\hline C183 & C184 & $1.36(2)$ \\
\hline C184 & C185 & $1.34(3)$ \\
\hline C185 & C186 & $1.37(3)$ \\
\hline C188 & C189 & $1.50(3)$ \\
\hline C189 & C190 & $1.36(3)$ \\
\hline C190 & C191 & $1.34(3)$ \\
\hline C191 & C192 & $1.45(3)$ \\
\hline C192 & C193 & $1.40(3)$ \\
\hline P1 & F5 & $1.558(11)$ \\
\hline $\mathrm{P} 1$ & $\mathrm{~F} 2$ & $1.608(11)$ \\
\hline $\mathrm{P} 1$ & $\mathrm{~F} 1$ & $1.523(11)$ \\
\hline $\mathrm{P} 1$ & $\mathrm{~F} 4$ & $1.573(11)$ \\
\hline P1 & F3 & $1.513(11)$ \\
\hline $\mathrm{P} 1$ & F6 & $1.553(11)$ \\
\hline $\mathrm{P} 2$ & F7 & $1.514(18)$ \\
\hline P2 & F8 & $1.551(18)$ \\
\hline
\end{tabular}




\begin{tabular}{lll}
\hline Atom & Atom & Length/A \\
\hline P2 & F9 & $1.594(16)$ \\
P2 & F10 & $1.627(16)$ \\
P2 & F11 & $1.56(2)$ \\
P2 & F12 & $1.497(18)$ \\
P3 & F13 & $1.565(12)$ \\
P3 & F14 & $1.598(11)$ \\
P3 & F15 & $1.531(11)$ \\
P3 & F16 & $1.569(12)$ \\
P3 & F17 & $1.515(12)$ \\
P3 & F18 & $1.533(12)$ \\
P4 & F19 & $1.60(3)$ \\
P4 & F20 & $1.79(3)$ \\
P4 & F21 & $1.64(2)$ \\
P4 & F22 & $1.39(2)$ \\
P4 & F23 & $1.56(3)$ \\
P4 & F24 & $1.41(2)$ \\
P5 & F25 & $1.53(2)$ \\
P5 & F26 & $1.53(2)$ \\
P5 & F27 & $1.51(2)$ \\
P5 & F28 & $1.52(2)$ \\
P5 & F29 & $1.564(18)$ \\
P5 & F30 & $1.59(2)$ \\
P6 & F31 & $1.519(12)$ \\
P6 & F32 & $1.589(11)$ \\
P6 & F33 & $1.527(12)$ \\
P6 & F34 & $1.555(11)$ \\
P6 & F35 & $1.545(11)$ \\
P6 & F36 & $1.562(12)$ \\
P7 & F37 & $1.520(13)$ \\
P7 & F38 & $1.602(13)$ \\
P7 & F39 & $1.528(13)$ \\
P7 & F40 & $1.558(13)$ \\
P7 & F41 & $1.574(13)$ \\
P7 & F42 & $1.534(13)$ \\
P8 & F43 & $1.529(13)$ \\
P8 & F44 & $1.596(13)$ \\
& &
\end{tabular}

\begin{tabular}{lll}
\hline Atom & Atom & Length/A \\
\hline P8 & F45 & $1.521(13)$ \\
P8 & F46 & $1.570(14)$ \\
P8 & F47 & $1.572(13)$ \\
P8 & F48 & $1.531(13)$ \\
P8A & F43A & $1.524(14)$ \\
P8A & F44A & $1.598(13)$ \\
P8A & F45A & $1.509(13)$ \\
P8A & F46A & $1.578(14)$ \\
P8A & F47A & $1.572(14)$ \\
P8A & F48A & $1.531(14)$ \\
P9 & F49 & $1.514(13)$ \\
P9 & F50 & $1.607(13)$ \\
P9 & F51 & $1.515(13)$ \\
P9 & F52 & $1.566(14)$ \\
P9 & F53 & $1.561(14)$ \\
P9 & F54 & $1.549(14)$ \\
P10 & F55 & $1.506(13)$ \\
P10 & F56 & $1.617(13)$ \\
P10 & F57 & $1.511(13)$ \\
P10 & F58 & $1.571(13)$ \\
P10 & F59 & $1.561(13)$ \\
P10 & F60 & $1.548(13)$ \\
P11 & F61 & $1.493(13)$ \\
P11 & F62 & $1.630(13)$ \\
P11 & F63 & $1.532(13)$ \\
P11 & F64 & $1.562(13)$ \\
P11 & F65 & $1.564(13)$ \\
P11 & F66 & $1.540(14)$ \\
P12 & F67 & $1.565(14)$ \\
P12 & F68 & $1.639(13)$ \\
P12 & F69 & $1.483(13)$ \\
P12 & F70 & $1.586(14)$ \\
P12 & F71 & $1.508(14)$ \\
P12 & F72 & $1.535(14)$ \\
& & \\
& & \\
& & \\
& & \\
P5) \\
\end{tabular}

Table S5: Bond Angles in ${ }^{\circ}$ for PL15004.

\begin{tabular}{llll}
\hline Atom & Atom & Atom & \multicolumn{1}{c}{ Angle $^{\circ}$} \\
\hline N1 & Co1 & N2 & $82.1(7)$ \\
N7 & Co1 & N1 & $93.3(7)$ \\
N7 & Co1 & N2 & $174.9(7)$ \\
N8 & Co1 & N1 & $90.6(7)$ \\
N8 & Co1 & N2 & $95.7(7)$ \\
N8 & Co1 & N7 & $81.9(7)$ \\
N8 & Co1 & N13 & $175.1(7)$ \\
N13 & Co1 & N1 & $91.6(7)$ \\
N13 & Co1 & N2 & $88.9(6)$ \\
N13 & Co1 & N7 & $93.6(7)$ \\
N14 & Co1 & N1 & $173.7(8)$ \\
N14 & Co1 & N2 & $95.7(7)$ \\
N14 & Co1 & N7 & $89.1(7)$ \\
N14 & Co1 & N8 & $95.5(7)$ \\
N14 & Co1 & N13 & $82.4(8)$ \\
N4 & Co2 & N20 & $174.3(7)$ \\
N4 & Co2 & N25 & $95.7(7)$ \\
N5 & Co2 & N4 & $81.3(8)$ \\
N5 & Co2 & N19 & $85.9(8)$ \\
N5 & Co2 & N20 & $93.9(7)$ \\
N5 & Co2 & N25 & $177.0(8)$ \\
N5 & Co2 & N26 & $97.5(8)$ \\
& & &
\end{tabular}

\begin{tabular}{llll}
\hline Atom & Atom & Atom & \multicolumn{1}{c}{ Angle $^{\circ}$} \\
\hline N19 & Co2 & N4 & $93.0(7)$ \\
N19 & Co2 & N20 & $83.5(8)$ \\
N19 & Co2 & N25 & $94.9(8)$ \\
N20 & Co2 & N25 & $89.1(7)$ \\
N26 & Co2 & N4 & $90.2(7)$ \\
N26 & Co2 & N19 & $175.6(8)$ \\
N26 & Co2 & N20 & $93.5(7)$ \\
N26 & Co2 & N25 & $81.8(8)$ \\
N11 & Co3 & N31 & $87.9(7)$ \\
N11 & Co3 & N10 & $82.3(7)$ \\
N11 & Co3 & N22 & $174.6(7)$ \\
N23 & Co3 & N11 & $96.8(7)$ \\
N23 & Co3 & N31 & $174.9(7)$ \\
N23 & Co3 & N10 & $89.4(6)$ \\
N23 & Co3 & N22 & $82.0(7)$ \\
N32 & Co3 & N11 & $97.6(7)$ \\
N32 & Co3 & N23 & $94.6(7)$ \\
N32 & Co3 & N31 & $82.9(7)$ \\
N32 & Co3 & N10 & $176.0(6)$ \\
N32 & Co3 & N22 & $87.7(6)$ \\
N10 & Co3 & N31 & $93.2(6)$ \\
N22 & Co3 & N31 & $93.5(6)$ \\
& & &
\end{tabular}




\begin{tabular}{|c|c|c|c|}
\hline Atom & Atom & Atom & Angle $/^{\circ}$ \\
\hline N22 & $\mathrm{Co} 3$ & N10 & $92.4(6)$ \\
\hline N16 & $\mathrm{Co} 4$ & N34 & $97.1(7)$ \\
\hline N17 & $\mathrm{Co} 4$ & N16 & $80.1(7)$ \\
\hline N17 & $\mathrm{Co} 4$ & N34 & $177.2(7)$ \\
\hline $\mathrm{N} 28$ & $\mathrm{Co} 4$ & N16 & $92.9(7)$ \\
\hline N28 & $\mathrm{Co} 4$ & N17 & $88.3(7)$ \\
\hline N28 & $\mathrm{Co} 4$ & N34 & $92.3(7)$ \\
\hline N28 & $\mathrm{Co} 4$ & N35 & $174.3(7)$ \\
\hline N29 & $\mathrm{Co} 4$ & N16 & $173.8(7)$ \\
\hline N29 & $\mathrm{Co} 4$ & N17 & $95.0(7)$ \\
\hline N29 & $\mathrm{Co} 4$ & N28 & $83.1(7)$ \\
\hline N29 & $\mathrm{Co} 4$ & N34 & $87.8(6)$ \\
\hline N29 & $\mathrm{Co} 4$ & N35 & $94.3(7)$ \\
\hline N35 & $\mathrm{Co} 4$ & N16 & $90.1(7)$ \\
\hline N35 & $\mathrm{Co} 4$ & N17 & $96.9(7)$ \\
\hline N35 & $\mathrm{Co} 4$ & N34 & $82.6(7)$ \\
\hline C9 & N1 & Co1 & $113.3(14)$ \\
\hline C13 & N1 & Co1 & $126.2(15)$ \\
\hline C13 & N1 & C9 & $120.4(18)$ \\
\hline $\mathrm{C} 1$ & $\mathrm{~N} 2$ & Co1 & $140.0(16)$ \\
\hline $\mathrm{C} 1$ & N2 & $\mathrm{C} 8$ & $110.3(19)$ \\
\hline $\mathrm{C} 8$ & N2 & Co1 & $109.7(15)$ \\
\hline C6 & N3 & $\mathrm{C} 7$ & $123(2)$ \\
\hline $\mathrm{C} 8$ & N3 & C6 & $110.7(18)$ \\
\hline $\mathrm{C} 8$ & N3 & $\mathrm{C} 7$ & $126(2)$ \\
\hline $\mathrm{C} 28$ & N4 & $\mathrm{Co} 2$ & $115.0(15)$ \\
\hline $\mathrm{C} 28$ & N4 & $\mathrm{C} 32$ & $119.8(19)$ \\
\hline C32 & N4 & $\mathrm{Co} 2$ & $125.1(15)$ \\
\hline $\mathrm{C} 20$ & N5 & $\mathrm{Co} 2$ & $139.3(17)$ \\
\hline $\mathrm{C} 27$ & N5 & $\mathrm{Co} 2$ & $115.5(16)$ \\
\hline $\mathrm{C} 27$ & N5 & $\mathrm{C} 20$ & $102(2)$ \\
\hline $\mathrm{C} 26$ & N6 & $\mathrm{C} 25$ & $127(2)$ \\
\hline $\mathrm{C} 27$ & N6 & $\mathrm{C} 25$ & $104.0(19)$ \\
\hline $\mathrm{C} 27$ & N6 & $\mathrm{C} 26$ & $129(2)$ \\
\hline C41 & N7 & Col & $114.2(15)$ \\
\hline $\mathrm{C} 45$ & N7 & Col & $126.6(16)$ \\
\hline $\mathrm{C} 45$ & N7 & C41 & $118.9(19)$ \\
\hline C33 & N8 & Co1 & $136.0(15)$ \\
\hline $\mathrm{C} 40$ & N8 & Co1 & $115.5(14)$ \\
\hline $\mathrm{C} 40$ & N8 & $\mathrm{C} 33$ & $106.5(17)$ \\
\hline C38 & N9 & C39 & $127(2)$ \\
\hline $\mathrm{C} 40$ & N9 & C38 & $106.9(19)$ \\
\hline $\mathrm{C} 40$ & N9 & C39 & $126(2)$ \\
\hline C52 & N11 & $\mathrm{Co} 3$ & $138.6(15)$ \\
\hline C59 & N11 & $\mathrm{Co} 3$ & $114.8(15)$ \\
\hline C59 & N11 & C52 & $106.4(18)$ \\
\hline C57 & N12 & C58 & $131(2)$ \\
\hline C57 & N12 & C59 & $102.5(18)$ \\
\hline C59 & N12 & C58 & $126(2)$ \\
\hline C73 & N13 & Co1 & $112.8(15)$ \\
\hline C73 & N13 & C77 & $120.0(18)$ \\
\hline $\mathrm{C} 77$ & N13 & Co1 & $127.1(14)$ \\
\hline C65 & N14 & Co1 & $138.1(16)$ \\
\hline $\mathrm{C} 72$ & N14 & Co1 & $117.1(17)$ \\
\hline $\mathrm{C} 72$ & N14 & C65 & $105(2)$ \\
\hline $\mathrm{C} 70$ & N15 & C71 & $125(2)$ \\
\hline $\mathrm{C} 72$ & N15 & $\mathrm{C} 70$ & $98(2)$ \\
\hline $\mathrm{C} 72$ & N15 & C71 & $136(2)$ \\
\hline C92 & N16 & $\mathrm{Co} 4$ & $118.7(14)$ \\
\hline C92 & N16 & C96 & $118.0(18)$ \\
\hline C96 & N16 & $\mathrm{Co} 4$ & $123.2(14)$ \\
\hline C84 & N17 & $\mathrm{Co} 4$ & $136.3(15)$ \\
\hline C91 & N17 & $\mathrm{Co} 4$ & $116.0(16)$ \\
\hline C91 & N17 & $\mathrm{C} 84$ & $107.6(19)$ \\
\hline
\end{tabular}

\begin{tabular}{|c|c|c|c|}
\hline Atom & Atom & Atom & Angle $/^{\circ}$ \\
\hline $\mathrm{C} 89$ & N18 & C90 & $129(2)$ \\
\hline C91 & N18 & C89 & $102(2)$ \\
\hline C91 & N18 & C90 & $127(2)$ \\
\hline $\mathrm{C} 105$ & N19 & $\mathrm{Co} 2$ & $118(2)$ \\
\hline C109 & N19 & $\mathrm{Co} 2$ & $126.6(17)$ \\
\hline C109 & N19 & C105 & $114(2)$ \\
\hline C104 & $\mathrm{N} 21$ & C103 & $137(3)$ \\
\hline C102 & N21 & C104 & 108.0 \\
\hline C102 & N21 & C103 & 115(3) \\
\hline N21 & C104 & C105 & $133(2)$ \\
\hline N20 & C104 & N21 & 108.0 \\
\hline N20 & C104 & C105 & $119(2)$ \\
\hline C104 & N20 & $\mathrm{Co} 2$ & $110.3(12)$ \\
\hline C104 & N20 & C97 & 108.0 \\
\hline C97 & N20 & $\mathrm{Co} 2$ & $141.7(12)$ \\
\hline C102 & C97 & N20 & 108.0 \\
\hline C98 & C97 & N20 & $127.6(19)$ \\
\hline C98 & C97 & C102 & $124(2)$ \\
\hline N21 & $\mathrm{C} 102$ & C97 & 108.0 \\
\hline N21 & C102 & C101 & $128(3)$ \\
\hline C97 & $\mathrm{C} 102$ & C101 & $124(3)$ \\
\hline C116 & N23 & Co3 & $140.9(15)$ \\
\hline C123 & N23 & $\mathrm{Co} 3$ & $114.0(14)$ \\
\hline C123 & N23 & C116 & $104.9(17)$ \\
\hline C121 & N24 & C122 & $125(2)$ \\
\hline C121 & $\mathrm{N} 24$ & C123 & $106.2(19)$ \\
\hline C123 & N24 & C122 & $129(2)$ \\
\hline C138 & N25 & $\mathrm{Co} 2$ & $118.3(16)$ \\
\hline C138 & N25 & C142 & $117(2)$ \\
\hline C142 & N25 & $\mathrm{Co} 2$ & $124.3(16)$ \\
\hline C137 & N27 & C136 & $129(2)$ \\
\hline C135 & N27 & C137 & $103.8(18)$ \\
\hline $\mathrm{C} 135$ & N27 & C136 & $127.0(19)$ \\
\hline N27 & C137 & C138 & $128(2)$ \\
\hline N26 & C137 & N27 & $111(2)$ \\
\hline N26 & C137 & C138 & $121(2)$ \\
\hline C137 & N26 & $\mathrm{Co} 2$ & $111.3(16)$ \\
\hline C130 & N26 & $\mathrm{Co} 2$ & $140.1(16)$ \\
\hline C130 & N26 & C137 & 108.6(19) \\
\hline N27 & C135 & C134 & $130.8(14)$ \\
\hline $\mathrm{N} 27$ & C135 & C130 & $109.1(14)$ \\
\hline C134 & C135 & C130 & 120.0 \\
\hline C135 & C134 & C133 & 120.0 \\
\hline C132 & C133 & C134 & 120.0 \\
\hline C133 & C132 & C131 & 120.0 \\
\hline C130 & C131 & C132 & 120.0 \\
\hline N26 & C130 & C135 & $107.7(15)$ \\
\hline N26 & C130 & C131 & $132.3(15)$ \\
\hline C131 & C130 & C135 & 120.0 \\
\hline C157 & N28 & $\mathrm{Co} 4$ & $117.3(15)$ \\
\hline C161 & N28 & $\mathrm{Co} 4$ & $126.8(15)$ \\
\hline C161 & $\mathrm{N} 28$ & C157 & $115.7(19)$ \\
\hline C156 & N29 & $\mathrm{Co} 4$ & $109.5(10)$ \\
\hline C149 & N29 & $\mathrm{Co} 4$ & $140.4(10)$ \\
\hline C149 & N29 & C156 & 108.0 \\
\hline N30 & C156 & N29 & 108.0 \\
\hline C157 & C156 & N29 & $120.4(17)$ \\
\hline C157 & C156 & N30 & $131.6(17)$ \\
\hline C156 & N30 & C155 & $126.3(18)$ \\
\hline C154 & N30 & C156 & 108.0 \\
\hline C154 & N30 & C155 & $125.2(18)$ \\
\hline N30 & C154 & C149 & 108.0 \\
\hline N30 & $\mathrm{C} 154$ & C153 & $122(2)$ \\
\hline C149 & C154 & C153 & $128(2)$ \\
\hline
\end{tabular}




\begin{tabular}{|c|c|c|c|}
\hline Atom & Atom & Atom & Angle $/^{\circ}$ \\
\hline N29 & C149 & C154 & 108.0 \\
\hline $\mathrm{C} 150$ & C149 & N29 & $135(2)$ \\
\hline C150 & C149 & C154 & $117(2)$ \\
\hline C149 & $\mathrm{C} 150$ & C151 & 121(3) \\
\hline C152 & C151 & C150 & $106(4)$ \\
\hline C151 & C152 & C153 & $132(4)$ \\
\hline C154 & C153 & C152 & 103(3) \\
\hline C170 & N31 & Co3 & $112.5(14)$ \\
\hline C174 & N31 & Co3 & $129.5(16)$ \\
\hline C174 & N31 & $\mathrm{C} 170$ & $118.0(19)$ \\
\hline C162 & N32 & Co3 & $140.2(15)$ \\
\hline C162 & N32 & C169 & $105.7(18)$ \\
\hline C169 & N32 & Co3 & $112.4(14)$ \\
\hline C168 & N33 & C167 & $125(2)$ \\
\hline C169 & N33 & $\mathrm{C} 167$ & $110(2)$ \\
\hline C169 & N33 & $\mathrm{C} 168$ & $126(2)$ \\
\hline C189 & N34 & $\mathrm{Co} 4$ & $114.3(15)$ \\
\hline C193 & N34 & $\mathrm{Co} 4$ & $122.6(15)$ \\
\hline C193 & N34 & C189 & $123.1(19)$ \\
\hline C181 & N35 & $\mathrm{Co} 4$ & $143.5(16)$ \\
\hline C188 & N35 & $\mathrm{Co} 4$ & $114.9(16)$ \\
\hline C188 & N35 & C181 & $101.5(19)$ \\
\hline C186 & N36 & C187 & $130(2)$ \\
\hline C188 & N36 & C186 & $107(2)$ \\
\hline C188 & N36 & C187 & $122(2)$ \\
\hline N2 & $\mathrm{C} 1$ & $\mathrm{C} 2$ & $134(2)$ \\
\hline $\mathrm{N} 2$ & $\mathrm{C} 1$ & C6 & $110(2)$ \\
\hline C6 & $\mathrm{C} 1$ & $\mathrm{C} 2$ & $115(2)$ \\
\hline $\mathrm{C} 1$ & $\mathrm{C} 2$ & C3 & $123(3)$ \\
\hline $\mathrm{C} 2$ & $\mathrm{C} 3$ & $\mathrm{C} 4$ & $116(3)$ \\
\hline $\mathrm{C} 5$ & $\mathrm{C} 4$ & C3 & $118(3)$ \\
\hline C6 & C5 & $\mathrm{C} 4$ & 121(3) \\
\hline $\mathrm{C} 1$ & C6 & N3 & $102(2)$ \\
\hline $\mathrm{C} 5$ & C6 & N3 & 131(2) \\
\hline $\mathrm{C} 5$ & C6 & $\mathrm{C} 1$ & $127(2)$ \\
\hline $\mathrm{N} 2$ & $\mathrm{C} 8$ & N3 & $106.2(19)$ \\
\hline N2 & $\mathrm{C} 8$ & C9 & $121(2)$ \\
\hline N3 & $\mathrm{C} 8$ & C9 & $133(2)$ \\
\hline N1 & C9 & $\mathrm{C} 8$ & $113.8(19)$ \\
\hline N1 & C9 & $\mathrm{C} 10$ & $118(2)$ \\
\hline $\mathrm{C} 10$ & C9 & $\mathrm{C} 8$ & $128(2)$ \\
\hline $\mathrm{C} 11$ & $\mathrm{C} 10$ & C9 & $118(2)$ \\
\hline C12 & $\mathrm{C} 11$ & $\mathrm{C} 10$ & 121(3) \\
\hline $\mathrm{C} 11$ & $\mathrm{C} 12$ & C14 & $124(2)$ \\
\hline C13 & $\mathrm{C} 12$ & $\mathrm{C} 11$ & $118(2)$ \\
\hline $\mathrm{C} 13$ & $\mathrm{C} 12$ & C14 & $118(2)$ \\
\hline N1 & $\mathrm{C} 13$ & $\mathrm{C} 12$ & $124(2)$ \\
\hline $\mathrm{C} 12$ & C14 & C15 & $114(2)$ \\
\hline C19 & $\mathrm{C} 14$ & C12 & $128(2)$ \\
\hline C19 & C14 & C15 & $118(2)$ \\
\hline $\mathrm{C} 16$ & $\mathrm{C} 15$ & $\mathrm{C} 14$ & $115(3)$ \\
\hline C15 & $\mathrm{C} 16$ & $\mathrm{C} 17$ & $128(3)$ \\
\hline $\mathrm{C} 16$ & $\mathrm{C} 17$ & $\mathrm{C} 18$ & $110(2)$ \\
\hline $\mathrm{C} 16$ & $\mathrm{C} 17$ & C31 & $128(2)$ \\
\hline C18 & $\mathrm{C} 17$ & C31 & $122(2)$ \\
\hline C19 & C18 & $\mathrm{C} 17$ & $127(3)$ \\
\hline C18 & C19 & $\mathrm{C} 14$ & 121(3) \\
\hline $\mathrm{C} 21$ & $\mathrm{C} 20$ & N5 & $129(3)$ \\
\hline $\mathrm{C} 25$ & $\mathrm{C} 20$ & N5 & $113(3)$ \\
\hline $\mathrm{C} 25$ & $\mathrm{C} 20$ & $\mathrm{C} 21$ & $117(3)$ \\
\hline $\mathrm{C} 22$ & $\mathrm{C} 21$ & $\mathrm{C} 20$ & 113(3) \\
\hline $\mathrm{C} 23$ & $\mathrm{C} 22$ & $\mathrm{C} 21$ & 119(3) \\
\hline $\mathrm{C} 24$ & $\mathrm{C} 23$ & $\mathrm{C} 22$ & $130(3)$ \\
\hline $\mathrm{C} 23$ & $\mathrm{C} 24$ & $\mathrm{C} 25$ & 106(3) \\
\hline
\end{tabular}

\begin{tabular}{|c|c|c|c|}
\hline Atom & Atom & Atom & Angle $/^{\circ}$ \\
\hline$\overline{\mathrm{C} 20}$ & $\mathrm{C} 25$ & N6 & $103(3)$ \\
\hline $\mathrm{C} 24$ & $\mathrm{C} 25$ & N6 & $124(3)$ \\
\hline $\mathrm{C} 24$ & $\mathrm{C} 25$ & $\mathrm{C} 20$ & $133(3)$ \\
\hline N5 & $\mathrm{C} 27$ & $\mathrm{C} 28$ & $113(2)$ \\
\hline N6 & $\mathrm{C} 27$ & N5 & $117(2)$ \\
\hline N6 & $\mathrm{C} 27$ & $\mathrm{C} 28$ & $130(2)$ \\
\hline N4 & $\mathrm{C} 28$ & $\mathrm{C} 27$ & $113(2)$ \\
\hline N4 & $\mathrm{C} 28$ & $\mathrm{C} 29$ & $125(2)$ \\
\hline $\mathrm{C} 29$ & $\mathrm{C} 28$ & $\mathrm{C} 27$ & $121(2)$ \\
\hline $\mathrm{C} 30$ & $\mathrm{C} 29$ & $\mathrm{C} 28$ & $110(2)$ \\
\hline $\mathrm{C} 29$ & $\mathrm{C} 30$ & C31 & $128(2)$ \\
\hline $\mathrm{C} 30$ & $\mathrm{C} 31$ & $\mathrm{C} 17$ & $127(2)$ \\
\hline C32 & C31 & $\mathrm{C} 17$ & $118(2)$ \\
\hline C32 & C31 & C30 & $115(2)$ \\
\hline N4 & $\mathrm{C} 32$ & C31 & $121(2)$ \\
\hline N8 & C33 & C34 & $135(2)$ \\
\hline N8 & $\mathrm{C} 33$ & C38 & $106(2)$ \\
\hline C38 & $\mathrm{C} 33$ & C34 & $119(2)$ \\
\hline C35 & C34 & $\mathrm{C} 33$ & $119(3)$ \\
\hline C34 & $\mathrm{C} 35$ & C36 & $123(3)$ \\
\hline $\mathrm{C} 35$ & $\mathrm{C} 36$ & C37 & $125(3)$ \\
\hline C38 & C37 & C36 & $109(3)$ \\
\hline N9 & C38 & $\mathrm{C} 33$ & $107(2)$ \\
\hline N9 & C38 & C37 & $127(2)$ \\
\hline C33 & C38 & C37 & $125(2)$ \\
\hline N8 & $\mathrm{C} 40$ & $\mathrm{C} 41$ & $114.7(19)$ \\
\hline N9 & $\mathrm{C} 40$ & N8 & $113.3(19)$ \\
\hline N9 & $\mathrm{C} 40$ & $\mathrm{C} 41$ & $132(2)$ \\
\hline N7 & C41 & $\mathrm{C} 40$ & $112.7(19)$ \\
\hline $\mathrm{C} 42$ & C41 & N7 & $121(2)$ \\
\hline $\mathrm{C} 42$ & $\mathrm{C} 41$ & $\mathrm{C} 40$ & $126(2)$ \\
\hline C41 & $\mathrm{C} 42$ & $\mathrm{C} 43$ & $116(2)$ \\
\hline $\mathrm{C} 44$ & $\mathrm{C} 43$ & $\mathrm{C} 42$ & $124(3)$ \\
\hline C43 & $\mathrm{C} 44$ & $\mathrm{C} 45$ & $114(2)$ \\
\hline C43 & $\mathrm{C} 44$ & C46 & $123(2)$ \\
\hline $\mathrm{C} 45$ & $\mathrm{C} 44$ & $\mathrm{C} 46$ & $123(2)$ \\
\hline N7 & $\mathrm{C} 45$ & C44 & $125(2)$ \\
\hline $\mathrm{C} 47$ & $\mathrm{C} 46$ & $\mathrm{C} 44$ & $123(2)$ \\
\hline $\mathrm{C} 47$ & $\mathrm{C} 46$ & C51 & $119(3)$ \\
\hline C51 & $\mathrm{C} 46$ & $\mathrm{C} 44$ & $118(2)$ \\
\hline $\mathrm{C} 48$ & $\mathrm{C} 47$ & $\mathrm{C} 46$ & $122(2)$ \\
\hline $\mathrm{C} 47$ & $\mathrm{C} 48$ & C49 & $123(2)$ \\
\hline $\mathrm{C} 50$ & C49 & $\mathrm{C} 48$ & $112(2)$ \\
\hline C64 & $\mathrm{C} 49$ & $\mathrm{C} 48$ & $126(2)$ \\
\hline C64 & C49 & $\mathrm{C} 50$ & $122(2)$ \\
\hline C49 & C50 & C51 & $125(3)$ \\
\hline $\mathrm{C} 46$ & C51 & $\mathrm{C} 50$ & $118(3)$ \\
\hline C53 & C52 & N11 & $128(2)$ \\
\hline C57 & C52 & N11 & $105(2)$ \\
\hline C57 & C52 & C53 & $127(2)$ \\
\hline C54 & C53 & C52 & $113(2)$ \\
\hline C53 & C54 & C55 & $123(3)$ \\
\hline C56 & C55 & C54 & $126(3)$ \\
\hline C55 & C56 & C57 & $112(3)$ \\
\hline N12 & C57 & C56 & $126(2)$ \\
\hline C52 & C57 & N12 & $114(2)$ \\
\hline C52 & C57 & C56 & $120(2)$ \\
\hline N11 & C59 & N12 & $112.0(19)$ \\
\hline N11 & C59 & $\mathrm{C} 60$ & $116.9(19)$ \\
\hline N12 & C59 & C60 & $131(2)$ \\
\hline C62 & C61 & C60 & 120.0 \\
\hline C61 & C62 & C64 & 120.0 \\
\hline C49 & C64 & C62 & $122.6(14)$ \\
\hline C49 & C64 & C63 & $117.4(14)$ \\
\hline
\end{tabular}




\begin{tabular}{|c|c|c|c|}
\hline Atom & Atom & Atom & Angle $/^{\circ}$ \\
\hline C63 & C64 & C62 & 120.0 \\
\hline N10 & C63 & C64 & 120.0 \\
\hline C63 & N10 & $\mathrm{Co} 3$ & $127.8(8)$ \\
\hline C63 & N10 & C60 & 120.0 \\
\hline C60 & N10 & $\mathrm{Co} 3$ & $112.2(8)$ \\
\hline C61 & C60 & C59 & $126.2(14)$ \\
\hline N10 & C60 & C59 & $113.5(14)$ \\
\hline N10 & $\mathrm{C} 60$ & C61 & 120.0 \\
\hline C66 & C65 & N14 & $130(2)$ \\
\hline $\mathrm{C} 70$ & C65 & N14 & $106(2)$ \\
\hline $\mathrm{C} 70$ & C65 & C66 & $124(2)$ \\
\hline C67 & C66 & C65 & $109(2)$ \\
\hline C68 & C67 & C66 & $130(3)$ \\
\hline C69 & C68 & C67 & $122(3)$ \\
\hline C68 & C69 & $\mathrm{C} 70$ & $112(3)$ \\
\hline N15 & C70 & C69 & $125(2)$ \\
\hline C65 & C70 & N15 & $112(2)$ \\
\hline C65 & $\mathrm{C} 70$ & C69 & $123(3)$ \\
\hline N14 & $\mathrm{C} 72$ & N15 & $119(2)$ \\
\hline N14 & $\mathrm{C} 72$ & C73 & $115(2)$ \\
\hline N15 & C72 & C73 & $126(2)$ \\
\hline N13 & C73 & $\mathrm{C} 72$ & $112(2)$ \\
\hline N13 & C73 & C74 & $123(2)$ \\
\hline C74 & C73 & C72 & $124(2)$ \\
\hline C73 & C74 & $\mathrm{C} 75$ & $114(2)$ \\
\hline C76 & $\mathrm{C} 75$ & C74 & $122(3)$ \\
\hline $\mathrm{C} 75$ & C76 & C78 & $119(2)$ \\
\hline C77 & C76 & $\mathrm{C} 75$ & $120(3)$ \\
\hline C77 & C76 & C78 & $120(2)$ \\
\hline $\mathrm{C} 76$ & C77 & N13 & $121(2)$ \\
\hline C79 & C78 & C76 & $121.8(18)$ \\
\hline C79 & C78 & $\mathrm{C} 83$ & 120.0 \\
\hline C83 & C78 & C76 & $118.2(18)$ \\
\hline $\mathrm{C} 80$ & C79 & $\mathrm{C} 78$ & 120.0 \\
\hline C79 & $\mathrm{C} 80$ & C81 & 120.0 \\
\hline $\mathrm{C} 80$ & C81 & $\mathrm{C} 95$ & $122.9(18)$ \\
\hline C82 & C81 & $\mathrm{C} 80$ & 120.0 \\
\hline C82 & C81 & $\mathrm{C} 95$ & $116.9(18)$ \\
\hline C81 & C82 & C83 & 120.0 \\
\hline C82 & $\mathrm{C} 83$ & C78 & 120.0 \\
\hline C85 & C84 & N17 & $136(2)$ \\
\hline C89 & $\mathrm{C} 84$ & N17 & $103(2)$ \\
\hline C89 & C84 & $\mathrm{C} 85$ & $121(2)$ \\
\hline C84 & $\mathrm{C} 85$ & C86 & $120(2)$ \\
\hline C87 & C86 & $\mathrm{C} 85$ & $115(2)$ \\
\hline C86 & C87 & $\mathrm{C} 88$ & $129(2)$ \\
\hline C87 & $\mathrm{C} 88$ & C89 & $112(2)$ \\
\hline N18 & C89 & C88 & $124(2)$ \\
\hline C84 & C89 & N18 & $113(2)$ \\
\hline C84 & C89 & C88 & $123(2)$ \\
\hline N17 & C91 & $\mathrm{C} 92$ & $114(2)$ \\
\hline N18 & C91 & N17 & $114(2)$ \\
\hline N18 & C91 & C92 & $132(2)$ \\
\hline N16 & C92 & C91 & $111(2)$ \\
\hline C93 & C92 & N16 & $125(2)$ \\
\hline C93 & $\mathrm{C} 92$ & C91 & $124(2)$ \\
\hline C92 & C93 & C94 & $121(2)$ \\
\hline C95 & C94 & C93 & $115(2)$ \\
\hline C94 & C95 & C81 & $120(2)$ \\
\hline C96 & C95 & C81 & $122(2)$ \\
\hline C96 & C95 & C94 & $117(2)$ \\
\hline C95 & C96 & N16 & $123(2)$ \\
\hline C100 & C101 & C102 & $106(4)$ \\
\hline C99 & $\mathrm{C} 100$ & C101 & $134(4)$ \\
\hline
\end{tabular}

\begin{tabular}{|c|c|c|c|}
\hline Atom & Atom & Atom & Angle $/^{\circ}$ \\
\hline$\overline{\mathrm{C} 100}$ & C99 & C98 & $118(3)$ \\
\hline C97 & C98 & C99 & $115(2)$ \\
\hline N19 & C105 & C104 & $109(3)$ \\
\hline C106 & $\mathrm{C} 105$ & N19 & $130(4)$ \\
\hline C106 & C105 & C104 & $120(4)$ \\
\hline C105 & C106 & C107 & $110(4)$ \\
\hline C108 & C107 & C106 & $118(3)$ \\
\hline $\mathrm{C} 107$ & $\mathrm{C} 108$ & C110 & $118(3)$ \\
\hline C109 & C108 & C107 & $121(3)$ \\
\hline C109 & $\mathrm{C} 108$ & C110 & $119(2)$ \\
\hline C108 & C109 & N19 & $123(2)$ \\
\hline C111 & C110 & C108 & $115.3(17)$ \\
\hline C111 & C110 & C115 & 120.0 \\
\hline C115 & C110 & C108 & $124.1(17)$ \\
\hline $\mathrm{C} 110$ & C111 & $\mathrm{C} 112$ & 120.0 \\
\hline C113 & $\mathrm{C} 112$ & C111 & 120.0 \\
\hline C112 & C113 & C114 & 120.0 \\
\hline C112 & C113 & C127 & $118.3(14)$ \\
\hline C114 & C113 & C127 & $121.7(14)$ \\
\hline C115 & C114 & C113 & 120.0 \\
\hline C114 & C115 & C110 & 120.0 \\
\hline C117 & C116 & N23 & $128(2)$ \\
\hline C121 & C116 & N23 & $109(2)$ \\
\hline C121 & C116 & C117 & $123(2)$ \\
\hline C116 & C117 & C118 & $113(2)$ \\
\hline C119 & C118 & C117 & 119(3) \\
\hline C118 & C119 & C120 & $127(3)$ \\
\hline C119 & C120 & C121 & $112(2)$ \\
\hline N24 & $\mathrm{C} 121$ & $\mathrm{C} 120$ & $127(2)$ \\
\hline C116 & C121 & N24 & $109(2)$ \\
\hline C116 & C121 & C120 & $124(2)$ \\
\hline N23 & C123 & N24 & $111.2(19)$ \\
\hline $\mathrm{N} 23$ & C123 & C124 & 119.7(19) \\
\hline N24 & C123 & C124 & $129(2)$ \\
\hline C125 & C124 & C123 & $131.0(14)$ \\
\hline $\mathrm{C} 125$ & $\mathrm{C} 124$ & N22 & 120.0 \\
\hline N22 & $\mathrm{C} 124$ & C123 & $108.9(14)$ \\
\hline C124 & C125 & C126 & 120.0 \\
\hline $\mathrm{C} 127$ & C126 & C125 & 120.0 \\
\hline C126 & C127 & C113 & $121.5(13)$ \\
\hline C126 & C127 & C128 & 120.0 \\
\hline C128 & $\mathrm{C} 127$ & C113 & $118.4(13)$ \\
\hline N22 & C128 & C127 & 120.0 \\
\hline C124 & N22 & Co3 & $115.3(9)$ \\
\hline C128 & N22 & $\mathrm{Co} 3$ & $124.6(9)$ \\
\hline C128 & N22 & C124 & 120.0 \\
\hline N25 & C138 & C137 & $107(2)$ \\
\hline N25 & C138 & C139 & $127(2)$ \\
\hline C139 & C138 & C137 & $126(2)$ \\
\hline C138 & C139 & C140 & $113(2)$ \\
\hline C139 & C140 & C141 & $126(3)$ \\
\hline C140 & C141 & C142 & $114(2)$ \\
\hline $\mathrm{C} 140$ & C141 & C143 & $123(2)$ \\
\hline $\mathrm{C} 142$ & C141 & C143 & $123(2)$ \\
\hline N25 & C142 & C141 & $123(2)$ \\
\hline C148 & C143 & C141 & $118.6(15)$ \\
\hline C148 & C143 & C144 & 120.0 \\
\hline $\mathrm{C} 144$ & C143 & C141 & $121.3(15)$ \\
\hline $\mathrm{C} 147$ & C148 & C143 & 120.0 \\
\hline C146 & C147 & C148 & 120.0 \\
\hline $\mathrm{C} 147$ & C146 & $\mathrm{C} 160$ & $117.8(15)$ \\
\hline $\mathrm{C} 145$ & C146 & C147 & 120.0 \\
\hline $\mathrm{C} 145$ & C146 & $\mathrm{C} 160$ & $122.1(15)$ \\
\hline C144 & C145 & C146 & 120.0 \\
\hline
\end{tabular}




\begin{tabular}{|c|c|c|c|}
\hline Atom & Atom & Atom & Angle $/^{\circ}$ \\
\hline$\overline{\mathrm{C} 145}$ & C144 & C143 & 120.0 \\
\hline N28 & C157 & C156 & $109(2)$ \\
\hline C158 & C157 & N28 & $125(3)$ \\
\hline C158 & C157 & C156 & $125(3)$ \\
\hline C157 & C158 & C159 & 119(3) \\
\hline C158 & C159 & C160 & $121(3)$ \\
\hline C146 & $\mathrm{C} 160$ & C159 & $124(2)$ \\
\hline C161 & $\mathrm{C} 160$ & C146 & $126(2)$ \\
\hline C161 & $\mathrm{C} 160$ & C159 & $110(2)$ \\
\hline N28 & $\mathrm{C} 161$ & C160 & $128(2)$ \\
\hline N32 & $\mathrm{C} 162$ & C163 & $131(2)$ \\
\hline N32 & $\mathrm{C} 162$ & $\mathrm{C} 167$ & $113(2)$ \\
\hline C163 & C162 & C167 & $115(2)$ \\
\hline $\mathrm{C} 162$ & C163 & C164 & $115(2)$ \\
\hline C165 & $\mathrm{C} 164$ & C163 & $123(3)$ \\
\hline C164 & $\mathrm{C} 165$ & C166 & $123(3)$ \\
\hline C167 & C166 & C165 & $114(3)$ \\
\hline C162 & C167 & N33 & $101(2)$ \\
\hline C166 & $\mathrm{C} 167$ & N33 & $130(3)$ \\
\hline C166 & $\mathrm{C} 167$ & C162 & $129(3)$ \\
\hline N33 & C169 & N32 & $110(2)$ \\
\hline N33 & C169 & C170 & $132(2)$ \\
\hline $\mathrm{C} 170$ & C169 & N32 & $118(2)$ \\
\hline C169 & $\mathrm{C} 170$ & N31 & $113(2)$ \\
\hline C169 & C170 & C171 & $129(2)$ \\
\hline C171 & C170 & N31 & $118(2)$ \\
\hline C172 & C171 & C170 & $118(2)$ \\
\hline C173 & C172 & C171 & $125(3)$ \\
\hline C172 & C173 & $\mathrm{C} 175$ & $127(2)$ \\
\hline C174 & C173 & C172 & $112(2)$ \\
\hline C174 & C173 & C175 & $122(2)$ \\
\hline N31 & C174 & C173 & $129(2)$ \\
\hline C176 & $\mathrm{C} 175$ & C173 & $118(2)$ \\
\hline C180 & C175 & C173 & $127(2)$ \\
\hline C180 & $\mathrm{C} 175$ & C176 & $116(3)$ \\
\hline $\mathrm{C} 175$ & C176 & C177 & 119(3) \\
\hline $\mathrm{C} 178$ & $\mathrm{C} 177$ & C176 & $123(3)$ \\
\hline C177 & C178 & C179 & 119(3) \\
\hline C177 & C178 & C192 & $122(3)$ \\
\hline C179 & C178 & C192 & $119(3)$ \\
\hline C178 & C179 & C180 & $116(3)$ \\
\hline $\mathrm{C} 175$ & C180 & C179 & $127(3)$ \\
\hline N35 & C181 & C182 & $128(2)$ \\
\hline C186 & C181 & N35 & $114(2)$ \\
\hline C186 & C181 & C182 & $119(2)$ \\
\hline C183 & C182 & C181 & $117(2)$ \\
\hline C182 & C183 & C184 & $124(2)$ \\
\hline C185 & C184 & C183 & $120(2)$ \\
\hline C184 & C185 & C186 & 119(3) \\
\hline C181 & C186 & N36 & $105(2)$ \\
\hline C181 & C186 & C185 & $123(2)$ \\
\hline C185 & C186 & N36 & $132(2)$ \\
\hline N35 & C188 & C189 & $114(2)$ \\
\hline N36 & C188 & N35 & $113(2)$ \\
\hline N36 & C188 & C189 & $133(2)$ \\
\hline N34 & C189 & C188 & 113(2) \\
\hline C190 & C189 & N34 & $118(2)$ \\
\hline C190 & C189 & C188 & $128(2)$ \\
\hline C191 & C190 & C189 & $122(2)$ \\
\hline C190 & C191 & C192 & $121(2)$ \\
\hline C191 & C192 & C178 & $123(2)$ \\
\hline C193 & C192 & C178 & $122(2)$ \\
\hline C193 & C192 & C191 & $115(2)$ \\
\hline N34 & C193 & C192 & $121(2)$ \\
\hline
\end{tabular}

\begin{tabular}{|c|c|c|c|}
\hline Atom & Atom & Atom & Angle $/^{\circ}$ \\
\hline$\overline{\mathrm{F} 5}$ & P1 & F2 & $89.5(7)$ \\
\hline F5 & $\mathrm{P} 1$ & F4 & $88.4(7)$ \\
\hline $\mathrm{F} 1$ & P1 & F5 & $90.2(7)$ \\
\hline F1 & $\mathrm{P} 1$ & $\mathrm{~F} 2$ & $177.7(8)$ \\
\hline $\mathrm{F} 1$ & P1 & F4 & $91.1(7)$ \\
\hline F1 & P1 & F6 & $90.7(7)$ \\
\hline F4 & $\mathrm{P} 1$ & $\mathrm{~F} 2$ & $86.7(7)$ \\
\hline F3 & $\mathrm{P} 1$ & F5 & $91.4(7)$ \\
\hline F3 & P1 & $\mathrm{F} 2$ & $89.3(7)$ \\
\hline F3 & P1 & $\mathrm{F} 1$ & $93.0(7)$ \\
\hline F3 & $\mathrm{P} 1$ & $\mathrm{~F} 4$ & $176.0(9)$ \\
\hline F3 & $\mathrm{P} 1$ & F6 & $91.5(8)$ \\
\hline F6 & P1 & F5 & $176.9(9)$ \\
\hline F6 & $\mathrm{P} 1$ & $\mathrm{~F} 2$ & $89.6(7)$ \\
\hline F6 & $\mathrm{P} 1$ & $\mathrm{~F} 4$ & $88.6(7)$ \\
\hline F7 & $\mathrm{P} 2$ & F8 & $172.2(13)$ \\
\hline F7 & $\mathrm{P} 2$ & F9 & $89.6(10)$ \\
\hline F7 & $\mathrm{P} 2$ & F10 & $88.5(9)$ \\
\hline F7 & P2 & F11 & $93.4(12)$ \\
\hline F8 & $\mathrm{P} 2$ & F9 & $87.3(9)$ \\
\hline F8 & $\mathrm{P} 2$ & F10 & $94.5(9)$ \\
\hline F8 & P2 & F11 & $79.4(11)$ \\
\hline F9 & $\mathrm{P} 2$ & F10 & $177.9(11)$ \\
\hline F11 & $\mathrm{P} 2$ & F9 & $89.5(10)$ \\
\hline F11 & $\mathrm{P} 2$ & F10 & $89.9(9)$ \\
\hline F12 & $\mathrm{P} 2$ & F7 & $97.5(11)$ \\
\hline F12 & P2 & F8 & $89.7(12)$ \\
\hline F12 & $\mathrm{P} 2$ & F9 & $90.9(9)$ \\
\hline F12 & $\mathrm{P} 2$ & F10 & $90.1(10)$ \\
\hline F12 & P2 & F11 & $169.1(13)$ \\
\hline F13 & P3 & F14 & $89.9(8)$ \\
\hline F13 & P3 & F16 & $87.0(8)$ \\
\hline F15 & P3 & F13 & $89.7(7)$ \\
\hline F15 & P3 & F14 & $178.4(9)$ \\
\hline F15 & P3 & F16 & $90.8(7)$ \\
\hline F15 & P3 & F18 & $90.8(8)$ \\
\hline F16 & P3 & F14 & $87.6(7)$ \\
\hline F17 & P3 & F13 & $90.4(8)$ \\
\hline F17 & P3 & F14 & $89.3(7)$ \\
\hline F17 & P3 & F15 & $92.3(8)$ \\
\hline F17 & P3 & F16 & $175.9(10)$ \\
\hline F17 & P3 & F18 & $92.2(9)$ \\
\hline F18 & P3 & F13 & $177.4(10)$ \\
\hline F18 & P3 & F14 & $89.5(7)$ \\
\hline F18 & P3 & F16 & $90.4(8)$ \\
\hline F19 & P4 & F20 & $167.0(15)$ \\
\hline F19 & $\mathrm{P} 4$ & F21 & $75.5(13)$ \\
\hline F21 & P4 & F20 & $91.6(13)$ \\
\hline F22 & $\mathrm{P} 4$ & F19 & $105.4(18)$ \\
\hline F22 & P4 & F20 & $87.5(17)$ \\
\hline F22 & $\mathrm{P} 4$ & F21 & $177.1(18)$ \\
\hline F22 & $\mathrm{P} 4$ & $\mathrm{~F} 23$ & $88.2(17)$ \\
\hline F22 & $\mathrm{P} 4$ & F24 & $84.6(15)$ \\
\hline F23 & P4 & F19 & $94.3(16)$ \\
\hline F23 & $\mathrm{P} 4$ & F20 & $86.9(16)$ \\
\hline F23 & P4 & F21 & $94.5(13)$ \\
\hline F24 & P4 & F19 & 95.1(19) \\
\hline F24 & $\mathrm{P} 4$ & F20 & $85.0(15)$ \\
\hline F24 & $\mathrm{P} 4$ & F21 & $92.6(15)$ \\
\hline F24 & $\mathrm{P} 4$ & F23 & $169(2)$ \\
\hline F25 & P5 & F29 & $91.5(11)$ \\
\hline F25 & P5 & F30 & $91.0(11)$ \\
\hline F26 & P5 & F25 & $176.9(12)$ \\
\hline F26 & P5 & F29 & $91.4(11)$ \\
\hline
\end{tabular}




\begin{tabular}{|c|c|c|c|c|c|c|c|}
\hline Atom & Atom & Atom & Angle $/^{\circ}$ & Atom & Atom & Atom & Angle $/^{\circ}$ \\
\hline F26 & P5 & F30 & $86.1(10)$ & $\overline{\mathrm{F} 45 \mathrm{~A}}$ & P8A & F48A & $92.4(10)$ \\
\hline $\mathrm{F} 27$ & P5 & $\mathrm{F} 25$ & $94.0(12)$ & F46A & P8A & F44A & $87.6(9)$ \\
\hline F27 & P5 & F26 & $87.2(11)$ & F47A & P8A & F44A & $89.5(9)$ \\
\hline $\mathrm{F} 27$ & P5 & F28 & $177.1(14)$ & F47A & P8A & F46A & $86.9(9)$ \\
\hline $\mathrm{F} 27$ & P5 & F29 & $87.0(13)$ & F48A & P8A & F44A & $89.7(9)$ \\
\hline $\mathrm{F} 27$ & P5 & F30 & $90.9(13)$ & F48A & P8A & F46A & $90.2(10)$ \\
\hline F28 & P5 & F25 & $87.1(12)$ & F48A & P8A & F47A & $177.0(12)$ \\
\hline F28 & P5 & F26 & $91.8(12)$ & F49 & P9 & F50 & $177.8(12)$ \\
\hline F28 & P5 & F29 & $90.3(12)$ & F49 & P9 & F51 & $92.5(10)$ \\
\hline F28 & P5 & F30 & $91.8(11)$ & F49 & P9 & F52 & $90.4(10)$ \\
\hline F29 & P5 & F30 & $176.8(12)$ & F49 & P9 & F53 & $92.2(10)$ \\
\hline F31 & P6 & F32 & 179.1(10) & F49 & P9 & F54 & $90.0(10)$ \\
\hline F31 & P6 & F33 & $91.8(9)$ & F51 & P9 & F50 & $89.5(9)$ \\
\hline F31 & P6 & F34 & $92.1(8)$ & F51 & P9 & F52 & $176.7(12)$ \\
\hline F31 & P6 & F35 & $90.5(8)$ & F51 & P9 & F53 & $90.3(10)$ \\
\hline F31 & P6 & F36 & $89.0(8)$ & F51 & P9 & F54 & $91.7(10)$ \\
\hline F33 & P6 & F32 & $88.6(8)$ & F52 & P9 & F50 & $87.7(9)$ \\
\hline F33 & P6 & F34 & $176.0(10)$ & F53 & P9 & F50 & $88.8(9)$ \\
\hline F33 & P6 & F35 & $91.0(8)$ & F53 & P9 & F52 & $88.0(9)$ \\
\hline F33 & P6 & F36 & $91.0(8)$ & F54 & P9 & F50 & $89.0(9)$ \\
\hline F34 & P6 & F32 & $87.5(7)$ & F54 & P9 & F52 & $89.9(10)$ \\
\hline F34 & P6 & F36 & $88.8(8)$ & F54 & P9 & F53 & $177.0(12)$ \\
\hline F35 & P6 & F32 & $90.3(7)$ & F55 & P10 & F56 & $177.7(12)$ \\
\hline F35 & P6 & F34 & $89.2(7)$ & F55 & P10 & F57 & $93.4(10)$ \\
\hline F35 & P6 & F36 & $177.9(9)$ & F55 & P10 & F58 & $89.9(9)$ \\
\hline F36 & P6 & F32 & $90.2(8)$ & F55 & P10 & F59 & $91.9(10)$ \\
\hline F37 & P7 & F38 & $178.6(12)$ & F55 & P10 & F60 & $91.5(10)$ \\
\hline F37 & P7 & F39 & $92.2(9)$ & F57 & P10 & F56 & $89.0(9)$ \\
\hline F37 & P7 & F40 & $90.8(9)$ & F57 & P10 & F58 & $176.7(12)$ \\
\hline F37 & P7 & F41 & $90.6(9)$ & F57 & P10 & F59 & $91.2(10)$ \\
\hline F37 & P7 & F42 & $90.6(9)$ & F57 & P10 & F60 & $91.8(10)$ \\
\hline F39 & P7 & F38 & $89.2(9)$ & F58 & P10 & F56 & $87.8(9)$ \\
\hline F39 & P7 & F40 & $176.1(12)$ & F59 & P10 & F56 & $88.2(9)$ \\
\hline F39 & P7 & F41 & $89.6(9)$ & F59 & P10 & F58 & $87.9(9)$ \\
\hline F39 & P7 & F42 & $91.3(9)$ & F60 & P10 & F56 & $88.4(9)$ \\
\hline F40 & P7 & F38 & $87.7(9)$ & F60 & P10 & F58 & $88.9(9)$ \\
\hline F40 & P7 & F41 & $88.0(9)$ & F60 & P10 & F59 & $175.4(12)$ \\
\hline F41 & P7 & F38 & $89.3(9)$ & F61 & P11 & F62 & $179.4(13)$ \\
\hline F42 & P7 & F38 & $89.4(9)$ & F61 & P11 & F63 & $93.0(10)$ \\
\hline F42 & P7 & F40 & $91.0(9)$ & F61 & P11 & F64 & $91.4(9)$ \\
\hline F42 & P7 & F41 & $178.4(11)$ & F61 & P11 & F65 & $92.5(10)$ \\
\hline F43 & P8 & F44 & $177.6(12)$ & F61 & P11 & F66 & $91.4(10)$ \\
\hline F43 & P8 & F46 & $90.1(9)$ & F63 & P11 & F62 & $87.6(9)$ \\
\hline F43 & P8 & F47 & $89.6(9)$ & F63 & P11 & F64 & $175.2(12)$ \\
\hline F43 & P8 & F48 & $90.5(9)$ & F63 & P11 & F65 & $90.6(9)$ \\
\hline F45 & P8 & F43 & $91.9(9)$ & F63 & P11 & F66 & $92.0(9)$ \\
\hline F45 & P8 & F44 & $90.1(9)$ & F64 & P11 & F62 & $88.0(9)$ \\
\hline F45 & P8 & F46 & $177.6(12)$ & F64 & P11 & F65 & $87.4(9)$ \\
\hline F45 & P8 & F47 & $90.9(9)$ & F65 & P11 & F62 & $87.6(9)$ \\
\hline F45 & P8 & F48 & $91.6(9)$ & F66 & P11 & F62 & $88.5(9)$ \\
\hline F46 & P8 & F44 & $87.8(9)$ & F66 & P11 & F64 & $89.7(9)$ \\
\hline F46 & P8 & F47 & $87.9(9)$ & F66 & P11 & F65 & $175.2(12)$ \\
\hline F47 & P8 & F44 & $89.1(9)$ & F67 & P12 & F68 & $88.0(9)$ \\
\hline F48 & P8 & F44 & $90.7(9)$ & F67 & P12 & F70 & $87.6(9)$ \\
\hline F48 & P8 & F46 & $89.7(9)$ & F69 & P12 & F67 & $92.0(10)$ \\
\hline F48 & P8 & F47 & $177.5(12)$ & F69 & P12 & F68 & $177.8(12)$ \\
\hline F43A & P8A & F44A & $177.1(12)$ & F69 & P12 & F70 & $92.0(10)$ \\
\hline $\mathrm{F} 43 \mathrm{~A}$ & P8A & F46A & $89.5(10)$ & F69 & P12 & F71 & $93.8(10)$ \\
\hline F43A & P8A & F47A & $90.2(10)$ & F69 & P12 & F72 & $92.5(10)$ \\
\hline F43A & P8A & F48A & $90.4(10)$ & F70 & P12 & F68 & $85.8(9)$ \\
\hline $\mathrm{F} 45 \mathrm{~A}$ & P8A & F43A & $93.3(10)$ & F71 & P12 & F67 & $90.1(10)$ \\
\hline $\mathrm{F} 45 \mathrm{~A}$ & P8A & F44A & $89.6(9)$ & F71 & P12 & F68 & $88.4(9)$ \\
\hline $\mathrm{F} 45 \mathrm{~A}$ & P8A & F46A & $176.2(12)$ & F71 & P12 & F70 & $173.8(12)$ \\
\hline F45A & P8A & F47A & $90.5(10)$ & F71 & P12 & F72 & $92.1(10)$ \\
\hline
\end{tabular}




\begin{tabular}{llll}
\hline Atom & Atom & Atom & \multicolumn{1}{c}{ Angle $^{\circ}$} \\
\hline F72 & P12 & F67 & $174.9(12)$ \\
F72 & P12 & F68 & $87.4(9)$ \\
F72 & P12 & F70 & $89.8(10)$
\end{tabular}


Table S6: Hydrogen Fractional Atomic Coordinates $\left(\times 10^{4}\right)$ and Equivalent Isotropic Displacement Parameters $\left(\AA^{2} \times 10^{3}\right)$ for PL15004. $U_{e q}$ is defined as $1 / 3$ of of the trace of the orthogonalised $U_{i j}$.

\begin{tabular}{|c|c|c|c|c|}
\hline Atom & & & & $U_{e q}$ \\
\hline H134 & 11984 & 6301 & 6351 & 61 \\
\hline H133 & 12445 & 5775 & 6554 & 46 \\
\hline H132 & 11666 & 5342 & 6730 & 37 \\
\hline H131 & 10427 & 5435 & 6704 & 49 \\
\hline H150 & 2377 & 8885 & 5801 & 120 \\
\hline H151 & 1021 & 8905 & 5783 & 165 \\
\hline H152 & 799 & 8542 & 5279 & 194 \\
\hline H153 & 965 & 7922 & 5375 & 167 \\
\hline $\mathrm{H} 2$ & 8339 & 9162 & 9332 & 84 \\
\hline H3 & 8461 & 9760 & 9425 & 82 \\
\hline H4 & 8998 & 10099 & 8953 & 101 \\
\hline H5 & 9257 & 9814 & 8453 & 67 \\
\hline H7A & 9425 & 9494 & 8018 & 118 \\
\hline H7B & 9765 & 9120 & 7962 & 118 \\
\hline $\mathrm{H} 7 \mathrm{C}$ & 8961 & 9199 & 7827 & 118 \\
\hline H10 & 9338 & 8622 & 7769 & 63 \\
\hline H11 & 9218 & 8068 & 7539 & 76 \\
\hline H13 & 8454 & 7736 & 8408 & 25 \\
\hline H15 & 8772 & 7604 & 7315 & 121 \\
\hline H16 & 8553 & 7036 & 7161 & 88 \\
\hline H18 & 9252 & 6703 & 8097 & 85 \\
\hline H19 & 9220 & 7232 & 8313 & 84 \\
\hline H21 & 9209 & 5095 & 6073 & 92 \\
\hline $\mathrm{H} 22$ & 9255 & 4475 & 6065 & 84 \\
\hline $\mathrm{H} 23$ & 9007 & 4176 & 6545 & 98 \\
\hline H24 & 8716 & 4402 & 7065 & 92 \\
\hline $\mathrm{H} 26 \mathrm{~A}$ & 8604 & 4761 & 7529 & 121 \\
\hline $\mathrm{H} 26 \mathrm{~B}$ & 8994 & 5095 & 7689 & 121 \\
\hline $\mathrm{H} 26 \mathrm{C}$ & 8171 & 5115 & 7561 & 121 \\
\hline H29 & 8904 & 5610 & 7759 & 27 \\
\hline H30 & 8914 & 6169 & 7938 & 62 \\
\hline H32 & 8933 & 6538 & 6972 & 40 \\
\hline H34 & 9887 & 8875 & 9001 & 78 \\
\hline H35 & 11093 & 8913 & 9065 & 97 \\
\hline H36 & 11774 & 8479 & 9299 & 131 \\
\hline H37 & 11297 & 7871 & 9373 & 105 \\
\hline H39A & 10515 & 7428 & 9421 & 85 \\
\hline H39B & 9728 & 7354 & 9554 & 85 \\
\hline H39C & 9899 & 7289 & 9158 & 85 \\
\hline H42 & 8694 & 7268 & 9403 & 65 \\
\hline H43 & 7499 & 7052 & 9300 & 113 \\
\hline H45 & 6983 & 7888 & 8783 & 43 \\
\hline H47 & 6612 & 6788 & 9019 & 64 \\
\hline H48 & 5479 & 6589 & 8949 & 44 \\
\hline H50 & 4821 & 7547 & 8671 & 86 \\
\hline H51 & 5921 & 7765 & 8898 & 81 \\
\hline H53 & 1528 & 5541 & 8374 & 48 \\
\hline H54 & 285 & 5501 & 8361 & 70 \\
\hline H55 & -426 & 5970 & 8452 & 78 \\
\hline H56 & 11 & 6494 & 8643 & 97 \\
\hline H58A & 1746 & 7120 & 8728 & 116 \\
\hline H58B & 1067 & 7068 & 8469 & 116 \\
\hline H58C & 996 & 6997 & 8873 & 116 \\
\hline H61 & 2595 & 7168 & 8750 & 87 \\
\hline H62 & 3759 & 7380 & 8843 & 100 \\
\hline
\end{tabular}




\begin{tabular}{|c|c|c|c|c|}
\hline Atom & & & & $U_{e q}$ \\
\hline H63 & 4565 & 6444 & 8601 & 28 \\
\hline H66 & 9058 & 8402 & 9715 & 45 \\
\hline H67 & 8968 & 8535 & 10299 & 67 \\
\hline H68 & 8003 & 8690 & 10560 & 64 \\
\hline H69 & 6974 & 8831 & 10262 & 100 \\
\hline H71A & 6113 & 8886 & 9834 & 138 \\
\hline H71B & 6020 & 9040 & 9452 & 138 \\
\hline $\mathrm{H} 71 \mathrm{C}$ & 5791 & 8650 & 9527 & 138 \\
\hline H74 & 5825 & 8746 & 8956 & 47 \\
\hline H75 & 5502 & 8678 & 8352 & 99 \\
\hline H77 & 7467 & 8387 & 8146 & 26 \\
\hline H79 & 5204 & 8421 & 7866 & 105 \\
\hline H80 & 4912 & 8416 & 7275 & 160 \\
\hline H82 & 6974 & 8639 & 7071 & 174 \\
\hline H83 & 7267 & 8643 & 7662 & 155 \\
\hline H85 & 3281 & 8413 & 5163 & 70 \\
\hline H86 & 3361 & 8448 & 4545 & 51 \\
\hline H87 & 4465 & 8550 & 4335 & 50 \\
\hline H88 & 5504 & 8648 & 4664 & 52 \\
\hline H90A & 6373 & 8627 & 5082 & 103 \\
\hline H90B & 6510 & 8763 & 5468 & 103 \\
\hline H90C & 6473 & 8357 & 5393 & 103 \\
\hline H93 & 6479 & 8595 & 5967 & 68 \\
\hline H94 & 6802 & 8653 & 6584 & 78 \\
\hline H96 & 4713 & 8441 & 6744 & 40 \\
\hline H101 & 8572 & 5340 & 4956 & 220 \\
\hline H100 & 9764 & 5482 & 4912 & 172 \\
\hline H99 & 10495 & 5740 & 5286 & 105 \\
\hline H98 & 10052 & 5907 & 5845 & 78 \\
\hline H10A & 7070 & 5197 & 5564 & 326 \\
\hline H10B & 7346 & 5399 & 5234 & 326 \\
\hline $\mathrm{H} 10 \mathrm{C}$ & 7708 & 5041 & 5348 & 326 \\
\hline H106 & 6881 & 5207 & 6087 & 190 \\
\hline H107 & 6030 & 5374 & 6568 & 145 \\
\hline H109 & 7575 & 5911 & 7018 & 48 \\
\hline H111 & 7232 & 5738 & 7486 & 92 \\
\hline H112 & 6614 & 5760 & 7999 & 90 \\
\hline H114 & 4739 & 5610 & 7461 & 98 \\
\hline H115 & 5357 & 5589 & 6947 & 96 \\
\hline H117 & 1964 & 6025 & 9119 & 82 \\
\hline H118 & 1698 & 6032 & 9758 & 85 \\
\hline H119 & 2419 & 5734 & 10121 & 86 \\
\hline H120 & 3564 & 5554 & 9995 & 61 \\
\hline $\mathrm{H} 12 \mathrm{~A}$ & 5088 & 5474 & 9351 & 122 \\
\hline H12B & 4858 & 5684 & 9685 & 122 \\
\hline $\mathrm{H} 12 \mathrm{C}$ & 4599 & 5294 & 9630 & 122 \\
\hline H125 & 5366 & 5464 & 8922 & 88 \\
\hline H126 & 6012 & 5467 & 8417 & 92 \\
\hline H128 & 4347 & 5939 & 7910 & 47 \\
\hline H13A & 10407 & 6954 & 6159 & 84 \\
\hline H13B & 11087 & 6895 & 6415 & 84 \\
\hline $\mathrm{H} 13 \mathrm{C}$ & 11115 & 6777 & 6021 & 84 \\
\hline H139 & 9555 & 7035 & 6153 & 47 \\
\hline H140 & 8418 & 7257 & 6104 & 81 \\
\hline H142 & 7583 & 6356 & 6337 & 43 \\
\hline H148 & 7376 & 7468 & 6222 & 92 \\
\hline H147 & 6222 & 7693 & 6126 & 93 \\
\hline H145 & 5494 & 6741 & 5862 & 59 \\
\hline H144 & 6647 & 6517 & 5957 & 90 \\
\hline
\end{tabular}




\begin{tabular}{|c|c|c|c|c|c|}
\hline Atom & & & & & $\boldsymbol{U}_{e q}$ \\
\hline H15A & 2499 & 7274 & 5589 & 130 & \\
\hline H15B & 1762 & 7384 & 5756 & 130 & \\
\hline $\mathrm{H} 15 \mathrm{C}$ & 1856 & 7423 & 5349 & 130 & \\
\hline H158 & 3458 & 7292 & 5501 & 93 & \\
\hline H159 & 4480 & 6973 & 5691 & 174 & \\
\hline H161 & 5199 & 7850 & 6145 & 44 & \\
\hline H163 & 2850 & 5227 & 8939 & 52 & \\
\hline H164 & 2691 & 4615 & 8987 & 71 & \\
\hline H165 & 2572 & 4272 & 8525 & 85 & \\
\hline H166 & 2585 & 4488 & 7969 & 95 & \\
\hline H16A & 2845 & 4815 & 7495 & 87 & \\
\hline H16B & 2518 & 5169 & 7346 & 87 & \\
\hline $\mathrm{H} 16 \mathrm{C}$ & 3366 & 5128 & 7408 & 87 & \\
\hline H171 & 2813 & 5671 & 7238 & 59 & \\
\hline H172 & 2866 & 6222 & 7016 & 93 & \\
\hline H174 & 3244 & 6599 & 7942 & 51 & \\
\hline H176 & 3058 & 6698 & 6824 & 109 & \\
\hline H177 & 3294 & 7262 & 6617 & 113 & \\
\hline H179 & 3012 & 7661 & 7550 & 81 & \\
\hline H180 & 2995 & 7094 & 7760 & 117 & \\
\hline H182 & 4319 & 9160 & 5579 & 43 & \\
\hline H183 & 4203 & 9720 & 5475 & 34 & \\
\hline H184 & 3613 & 10083 & 5831 & 55 & \\
\hline H185 & 3057 & 9865 & 6305 & 77 & \\
\hline H18A & 2600 & 9509 & 6783 & 160 & \\
\hline H18B & 2245 & 9134 & 6795 & 160 & \\
\hline $\mathrm{H} 18 \mathrm{C}$ & 2997 & 9205 & 6993 & 160 & \\
\hline H190 & 2627 & 8655 & 7028 & 58 & \\
\hline H191 & 2624 & 8129 & 7289 & 69 & \\
\hline H193 & 3742 & 7748 & 6490 & 36 & \\
\hline
\end{tabular}


1d $\cdot 12 \mathrm{PF}_{6}(\mathrm{CCDC} 1425919)$ Crystal Data and Experimental

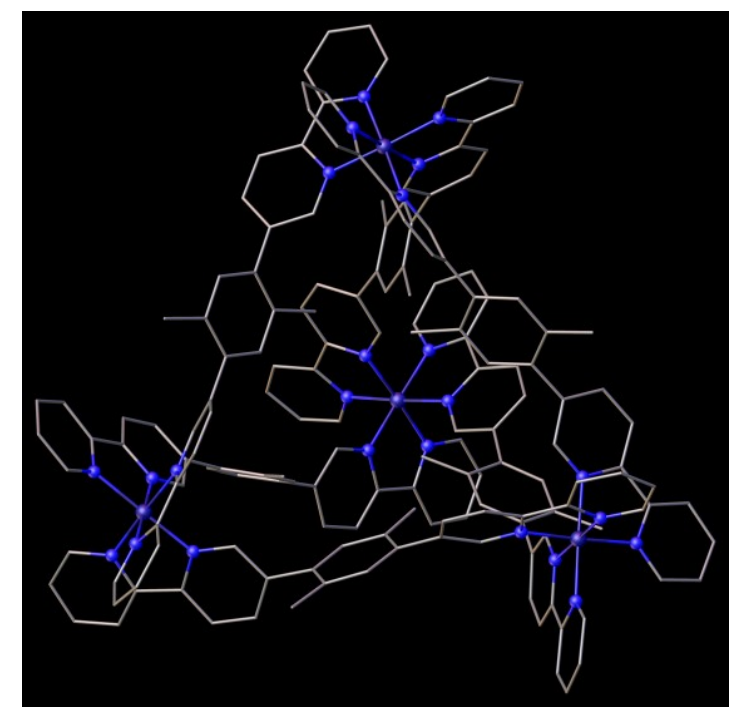

Experimental. Single yellow prism-shaped crystals of (PL15015-sq) were recrystallised from a mixture of acetonitrile and diisopropyl ether by vapour diffusion. A suitable crystal $(0.29 \times 0.19 \times 0.19)$ was selected and mounted on a MITIGEN holder in Paratone oil on a Rigaku Oxford Diffraction SuperNova diffractometer. The crystal was kept at $T=120.0 \mathrm{~K}$ during data collection. Using Olex2, ${ }^{[3]}$ the structure was solved with the ShelXT structure solution program, ${ }^{[5]}$ using the direct methods solution method. The model was refined with version of ShelXL using Least Squares minimisation. ${ }^{[5]}$

Crystal Data. $\mathrm{C}_{168} \mathrm{H}_{132} \mathrm{Co}_{4} \mathrm{~F}_{72} \mathrm{~N}_{24} \mathrm{P}_{12}, M_{r}=4462.33$, trigonal, R-3c (No. 167), $\mathrm{a}=20.5996(2) \AA, \mathrm{b}=20.5996(2) \AA, \mathrm{c}=$ $195.2193(18) \AA, \alpha=90^{\circ}, \quad \beta=90^{\circ}, \quad \gamma=120^{\circ}, \quad V=$ $71741.6(15) \AA^{3}, \quad T=120.0 \mathrm{~K}, \quad Z=12, \quad Z^{\prime}=0.33333$, $\mu\left(\mathrm{CuK}_{\alpha}\right)=3.803,97557$ reflections measured, 16050 unique $\left(R_{\text {int }}=0.0818\right)$ which were used in all calculations. The final $w R_{2}$ was 0.2877 (all data) and $R_{l}$ was 0.0911 (I $>2(\mathrm{I})$ ).

\begin{tabular}{|c|c|}
\hline Compound & PL15015-sq \\
\hline Formula & $\mathrm{C}_{168} \mathrm{H}_{132} \mathrm{Co}_{4} \mathrm{~F}_{72} \mathrm{~N}_{24} \mathrm{P}_{12}$ \\
\hline$D_{\text {calc. }} / \mathrm{g} \mathrm{cm}^{-3}$ & 1.239 \\
\hline$\mu / \mathrm{mm}^{-1}$ & 3.803 \\
\hline Formula Weight & 4462.33 \\
\hline Colour & yellow \\
\hline Shape & prism \\
\hline Max Size/mm & 0.29 \\
\hline Mid Size/mm & 0.19 \\
\hline Min Size/mm & 0.19 \\
\hline$T / \mathrm{K}$ & 120.0 \\
\hline Crystal System & trigonal \\
\hline Space Group & $\mathrm{R}-3 \mathrm{c}$ \\
\hline$a / \AA$ & $20.5996(2)$ \\
\hline$b / \AA$ & $20.5996(2)$ \\
\hline$c / \AA$ & $195.2193(18)$ \\
\hline$\alpha /^{\circ}$ & 90 \\
\hline$\beta 1^{\circ}$ & 90 \\
\hline$\gamma /^{\circ}$ & 120 \\
\hline $\mathrm{V} / \AA^{3}$ & $71741.6(15)$ \\
\hline$Z$ & 12 \\
\hline$Z^{\prime}$ & 0.33333 \\
\hline$\theta_{\min } 1^{\circ}$ & 3.069 \\
\hline$\theta_{\max } l^{\circ}$ & 75.084 \\
\hline Measured Refl. & 97557 \\
\hline Independent Refl. & 16050 \\
\hline Reflections Used & 11098 \\
\hline$R_{\text {int }}$ & 0.0818 \\
\hline Parameters & 872 \\
\hline Restraints & 526 \\
\hline Largest Peak & 1.762 \\
\hline Deepest Hole & -1.026 \\
\hline GooF & 1.069 \\
\hline$w R_{2}$ (all data) & 0.2877 \\
\hline$w R_{2}$ & 0.2689 \\
\hline$R_{1}$ (all data) & 0.1153 \\
\hline$R_{1}$ & 0.0911 \\
\hline
\end{tabular}




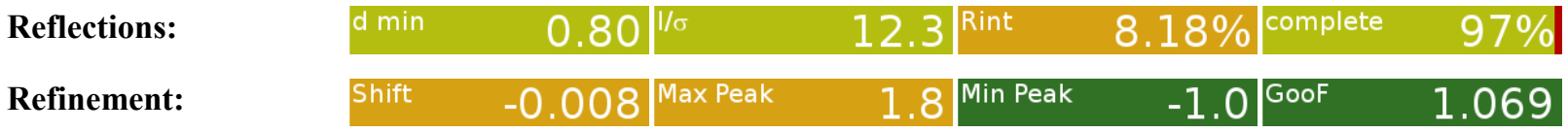

A yellow prism-shaped crystal with dimensions $0.29 \times 0.19 \times 0.19$ was mounted on a MITIGEN holder in Paratone oil. Data were collected using a Rigaku Oxford Diffraction SuperNova diffractometer equipped with an Oxford Cryosystems Cryostream 700+ low-temperature apparatus operating at $T=120.0 \mathrm{~K}$.

Data were measured using $\omega$ scans scans of $0.3^{\circ}$ per frame for $10.0 \mathrm{~s}$ using $\mathrm{CuK}_{\alpha}$ radiation (sealed X-ray tube, $50 \mathrm{kV}, 0.8 \mathrm{~mA}$ ). The total number of runs and images was based on the strategy calculation from the program CrysAlisPro (Agilent, V1.171.37.35e, 2014). The actually achieved resolution was $\theta=75.084$.

Cell parameters were retrieved using the CrysAlisPro (Agilent, V1.171.37.35e, 2014) software and refined using CrysAlisPro (Agilent, V1.171.37.35e, 2014) on 20459 reflections, 21 of the observed reflections. Data reduction was performed using the CrysAlisPro (Agilent, V1.171.37.35e, 2014) software, which corrects for Lorentz polarisation. The final completeness is 99.20 out to 75.084 in $\theta$. The absorption coefficient $(\mu)$ of this material is 3.803 and the minimum and maximum transmissions are 0.59974 and 1.00000 .

The structure was solved in the space group R-3c (\# 167) by direct methods using the ShelXT structure solution program and refined by Least Squares using version of ShelXL. ${ }^{[4-5]}$ All non-hydrogen atoms were refined anisotropically. Hydrogen atom positions were calculated geometrically and refined using the riding model. The crystals decompose instantly when removed from the mother liquor. An aliquot of ML plus crystals was transferred by pipette onto a microscope slide with shallow wells, a suitable crystal identified and quickly pushed into Paratone oil and mounted to minimise solvent loss and crystal decomposition. The SQUEEZE routine of PLATON was used to account for electron density pertaining to a lot of disordered solvent. A total of 6023 electrons per unit cell were handled by SQUEEZE. This equates to 30 molecules of acetonitrile per asymmetric unit. However, it is not at all clear from the (un-SQUEEZED) difference map that the residual solvent is acetonitrile. A mix of acetonitrile and diisopropyl ether was used in the crystallization, and other solvents including water were used in the synthesis. In the absence of any other information it was not thought appropriate to speculate on the identity of the squeezed solvent and include it in the total chemical formula. The moiety formula is thus presented as "compound + solvent". The methyl groups attached to one central aryl ring were modelled as disordered over two sites, as evidenced by peaks in a difference Fourier map. Bond distance and displacement ellipsoid restraints were used. Geometric similarity and displacement ellipsoid restraints were used to control some of the PF6 anions. Some anions lie on crystallographic symmetry elements (e.g. P4 and P5). In the case of P5 the anion is disordered about the threefold axis. Occupancies of the anions were adjusted for charge balance (one complete tetrahedron carries a charge of $12+$ ).

The value of $Z^{\prime}$ is 0.33333 . 


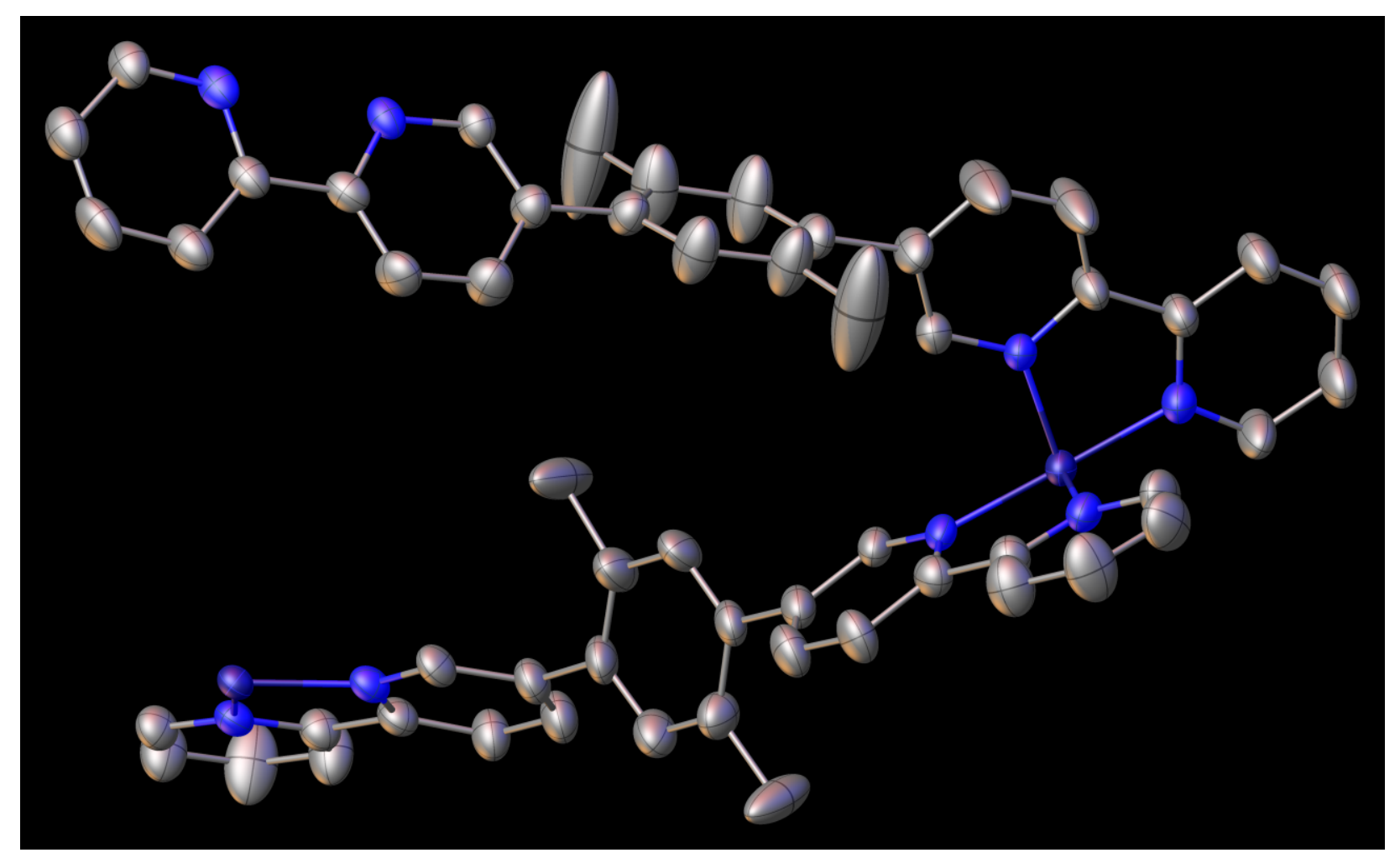

Figure S61: The asymmetric part of the cation. Displacement ellipsoids are at the 50pc probability level. H atoms and disorder are not shown.

\section{Reflection Statistics}

Total reflections (after filtering) 97606

Completeness $\quad 0.974$

hklsub $>\max </$ sub $>$ collected $\quad(25,24,244)$

hkl max used

Lim $d_{\max }$ collected

$\mathrm{d}_{\max }$ used

$(0,25,244)$

100.0

14.4

Friedel pairs

Inconsistent equivalents

Rsigma

17220

479

0.0512

Omitted reflections

Multiplicity

0

Removed systematic absences

$\begin{array}{ll}\text { Unique reflections } & 16050 \\ \text { Mean } \mathrm{I} / \sigma & 12.32 \\ \mathrm{hkl} / \mathrm{sub}>\text { min }</ \text { sub }>\text { collected } & (-20,-25,-238) \\ \mathrm{hkl} l_{\min } \text { used } & (-21,0,0) \\ \text { Lim dmin collected } & 0.77 \\ \mathrm{~d}_{\text {min }} \text { used } & 0.8 \\ \text { Friedel pairs merged } & 1 \\ \mathrm{R}_{\text {int }} & 0.0818 \\ \text { Intensity transformed } & 0 \\ \text { Omitted by user (OMIT hkl) } & 49 \\ \text { Maximum multiplicity } & 21 \\ \text { Filtered off (Shel/OMIT) } & 0\end{array}$

Table S7: Fractional Atomic Coordinates $\left(\times 10^{4}\right)$ and Equivalent Isotropic Displacement Parameters $\left(\AA^{2} \times 10^{3}\right)$ for PL15015_sq. $U_{e q}$ is defined as $1 / 3$ of the trace of the orthogonalised $U_{i j}$.

\begin{tabular}{lclll}
\hline Atom & \multicolumn{1}{c}{$\mathbf{x}$} & \multicolumn{1}{c}{$\mathbf{y}$} & $\mathbf{z}$ & $\boldsymbol{U}_{\boldsymbol{e q}}$ \\
\hline Co1 & $10500.3(4)$ & $6837.7(4)$ & $6979.4(2)$ & $31.38(19)$ \\
Co2 & 6667 & 3333 & $7530.6(2)$ & $28.8(3)$ \\
N1 & $11015(2)$ & $6836(2)$ & $6897.2(2)$ & $39.2(8)$ \\
N2 & $9646.0(18)$ & $6451.1(19)$ & $6919.3(2)$ & $31.9(7)$ \\
N3 & $4126(2)$ & $4510(2)$ & $7001.4(2)$ & $33.1(7)$ \\
N4 & $2794(2)$ & $4151(2)$ & $7040.0(2)$ & $35.3(8)$ \\
N5 & $10609.8(19)$ & $7812(2)$ & $6961.8(2)$ & $39.4(8)$ \\
N6 & $9958.9(18)$ & $6869(2)$ & $7060.0(2)$ & $32.8(7)$ \\
N7 & $7583(2)$ & $3667.9(19)$ & $7480.0(2)$ & $33.3(7)$ \\
N8 & $7044(2)$ & $2797.2(19)$ & $7583.4(2)$ & $34.1(7)$ \\
C1 & $11729(3)$ & $6996(4)$ & $6892.6(3)$ & $62.1(16)$ \\
C2 & $12040(3)$ & $6951(4)$ & $6831.4(3)$ & $64.1(16)$ \\
C3 & $11612(3)$ & $6742(4)$ & $6772.7(2)$ & $59.5(15)$
\end{tabular}




\begin{tabular}{|c|c|c|c|c|}
\hline Atom & $\mathbf{x}$ & $\mathbf{y}$ & $\mathbf{z}$ & $U_{e q}$ \\
\hline$\overline{\mathrm{C} 4}$ & $10863(3)$ & $6565(4)$ & $6777.1(2)$ & $57.1(14)$ \\
\hline $\mathrm{C} 5$ & $10586(3)$ & $6610(3)$ & $6840.2(2)$ & $41.6(10)$ \\
\hline C6 & $9810(3)$ & $6429(3)$ & $6852.0(2)$ & $42.2(10)$ \\
\hline $\mathrm{C} 7$ & $9277(4)$ & $6233(5)$ & $6802.6(3)$ & $84(2)$ \\
\hline $\mathrm{C} 8$ & $8554(4)$ & $6051(5)$ & $6820.7(3)$ & $85(2)$ \\
\hline C9 & $8376(2)$ & $6053(3)$ & $6888.8(2)$ & $44.2(11)$ \\
\hline $\mathrm{C} 10$ & $8941(2)$ & $6268(2)$ & $6936.5(2)$ & $35.1(9)$ \\
\hline C11 & $7611(3)$ & $5864(3)$ & $6913.3(2)$ & $42.5(10)$ \\
\hline $\mathrm{C} 12$ & $7395(3)$ & $6385(3)$ & $6914.8(4)$ & $72.2(19)$ \\
\hline C13 & $6689(3)$ & $6166(3)$ & $6942.1(4)$ & $67.7(17)$ \\
\hline C14 & $6198(3)$ & $5459(3)$ & $6964.5(2)$ & $42(1)$ \\
\hline C15 & $6412(4)$ & $4918(4)$ & $6961.3(4)$ & $82(2)$ \\
\hline $\mathrm{C} 16$ & $7134(4)$ & $5148(4)$ & $6935.3(5)$ & $89(3)$ \\
\hline $\mathrm{C} 17$ & $7924(6)$ & $7192(5)$ & $6893.7(8)$ & $180(7)$ \\
\hline C18 & $5953(6)$ & $4144(6)$ & $6985.6(9)$ & 219(9) \\
\hline C19 & $4801(2)$ & $4666(2)$ & $6975.6(2)$ & $35.0(9)$ \\
\hline $\mathrm{C} 20$ & $5469(2)$ & $5305(3)$ & $6993.3(2)$ & $38.9(9)$ \\
\hline $\mathrm{C} 21$ & $5430(3)$ & $5810(3)$ & $7038.9(3)$ & $48.9(11)$ \\
\hline $\mathrm{C} 22$ & $4746(3)$ & $5659(3)$ & $7064.4(2)$ & $47.2(11)$ \\
\hline $\mathrm{C} 23$ & $4103(3)$ & $5004(3)$ & $7045.9(2)$ & $36.2(9)$ \\
\hline $\mathrm{C} 24$ & $3347(2)$ & $4768(3)$ & $7072.0(2)$ & $35.6(9)$ \\
\hline $\mathrm{C} 25$ & $3196(3)$ & $5113(3)$ & $7126.1(2)$ & $46.2(11)$ \\
\hline $\mathrm{C} 26$ & $2464(3)$ & $4824(3)$ & $7146.2(2)$ & $53.3(13)$ \\
\hline $\mathrm{C} 27$ & 1897(3) & $4206(3)$ & $7113.9(2)$ & $50.0(12)$ \\
\hline $\mathrm{C} 28$ & 2078(3) & $3878(3)$ & $7059.8(2)$ & $41.4(10)$ \\
\hline $\mathrm{C} 29$ & $10936(3)$ & $8256(3)$ & $6907.3(2)$ & $51.2(12)$ \\
\hline C30 & $10920(4)$ & $8903(3)$ & 6896.1(3) & $64.8(16)$ \\
\hline C31 & $10549(4)$ & $9112(4)$ & $6942.3(3)$ & $72.3(18)$ \\
\hline C32 & $10208(4)$ & $8658(3)$ & 6999.3(3) & $61.5(14)$ \\
\hline C33 & $10251(3)$ & $8014(3)$ & $7007.9(2)$ & $42.2(10)$ \\
\hline C34 & $9943(2)$ & $7511(3)$ & $7065.9(2)$ & $38.0(9)$ \\
\hline C35 & $9666(3)$ & $7676(3)$ & $7125.2(2)$ & $51.2(12)$ \\
\hline C36 & $9413(3)$ & $7161(3)$ & $7178.3(2)$ & $51.2(12)$ \\
\hline $\mathrm{C} 37$ & $9435(2)$ & $6502(3)$ & $7172.9(2)$ & $41.6(10)$ \\
\hline C38 & $9706(2)$ & $6376(3)$ & $7111.9(2)$ & $35.5(9)$ \\
\hline C39 & $9201(2)$ & $5951(3)$ & $7229.3(2)$ & $40.8(10)$ \\
\hline $\mathrm{C} 42$ & $8771(3)$ & $4952(3)$ & $7338.9(2)$ & $44.9(11)$ \\
\hline $\mathrm{C} 40$ & $8635(3)$ & $5218(3)$ & $7221.4(2)$ & $52.8(13)$ \\
\hline $\mathrm{C} 41$ & $8425(3)$ & $4724(3)$ & $7276.2(2)$ & $57.4(14)$ \\
\hline $\mathrm{C} 43$ & $9359(3)$ & $5690(3)$ & $7346.7(2)$ & $54.7(13)$ \\
\hline $\mathrm{C} 44$ & $9566(3)$ & $6188(3)$ & $7292.6(3)$ & $51.4(12)$ \\
\hline $\mathrm{C} 45$ & $10249(7)$ & $6951(7)$ & $7305.4(6)$ & $92(5)$ \\
\hline C40A & $8635(3)$ & $5218(3)$ & $7221.4(2)$ & $52.8(13)$ \\
\hline C41A & $8425(3)$ & $4724(3)$ & $7276.2(2)$ & $57.4(14)$ \\
\hline C43A & $9359(3)$ & $5690(3)$ & $7346.7(2)$ & $54.7(13)$ \\
\hline C44A & $9566(3)$ & $6188(3)$ & $7292.6(3)$ & $51.4(12)$ \\
\hline $\mathrm{C} 45 \mathrm{~A}$ & $9852(10)$ & $6005(10)$ & $7412.5(7)$ & $102(7)$ \\
\hline C46 & $7748(8)$ & $3912(6)$ & $7258.7(5)$ & $90(5)$ \\
\hline C46A & $8152(14)$ & $4889(14)$ & $7158.3(7)$ & 199(17) \\
\hline C47 & $7823(3)$ & $4145(3)$ & $7425.7(2)$ & $38.4(9)$ \\
\hline $\mathrm{C} 48$ & $8544(3)$ & $4433(3)$ & $7398.2(2)$ & $42.7(10)$ \\
\hline C49 & $9021(3)$ & $4231(3)$ & $7427.6(2)$ & $51.1(12)$ \\
\hline C50 & $8775(3)$ & $3729(3)$ & $7483.2(2)$ & $47.8(11)$ \\
\hline C51 & $8053(2)$ & $3460(3)$ & $7507.0(2)$ & $37.7(9)$ \\
\hline C52 & $7721(3)$ & $2919(3)$ & $7563.4(2)$ & $38.0(9)$ \\
\hline C53 & $8058(3)$ & $2563(4)$ & 7594.7(3) & $59.3(15)$ \\
\hline C54 & $7683(4)$ & $2066(4)$ & $7647.6(4)$ & $84(2)$ \\
\hline C55 & $6992(3)$ & 1939(3) & $7668.0(3)$ & $58.8(14)$ \\
\hline C56 & $6680(3)$ & $2309(3)$ & $7634.4(2)$ & $40.9(10)$ \\
\hline P1 & $9324.9(8)$ & 4101.7(9) & $7732.3(2)$ & $54.8(4)$ \\
\hline F1 & $9387(2)$ & $4825(2)$ & $7766.1(2)$ & $93.2(13)$ \\
\hline $\mathrm{F} 2$ & $8426.3(18)$ & $3695(2)$ & $7739.9(2)$ & $77.5(11)$ \\
\hline F3 & $9256.6(19)$ & $4389(3)$ & $7658.9(2)$ & 86.1(13) \\
\hline $\mathrm{F} 4$ & $9253(2)$ & $3365(3)$ & $7699.4(2)$ & $94.2(13)$ \\
\hline
\end{tabular}




\begin{tabular}{lclll}
\hline Atom & $\mathbf{x}$ & \multicolumn{1}{c}{$\mathbf{y}$} & $\mathbf{z}$ & \multicolumn{1}{c}{$\boldsymbol{U}_{\boldsymbol{c}}$} \\
\hline F5 & $10205.9(18)$ & $4507(2)$ & $7724.9(2)$ & $76.9(11)$ \\
F6 & $9375(2)$ & $3792(2)$ & $7805.7(2)$ & $72.5(10)$ \\
P2 & $7887.6(12)$ & $7871.6(15)$ & $7100.2(2)$ & $122(1)$ \\
F7 & $7789(3)$ & $8551(3)$ & $7121.7(3)$ & $134.7(19)$ \\
F8 & $7413(4)$ & $7781(5)$ & $7035.5(4)$ & $184(3)$ \\
F9 & $8643(3)$ & $8405(4)$ & $7063.1(4)$ & $156(2)$ \\
F10 & $7999(4)$ & $7204(4)$ & $7074.3(5)$ & $191(3)$ \\
F11 & $8343(4)$ & $7905(5)$ & $7165.7(4)$ & $169(3)$ \\
F12 & $7166(4)$ & $7339(5)$ & $7138.7(6)$ & $213(4)$ \\
P3 & $11905(2)$ & $8911.7(17)$ & $6719.3(2)$ & $133.8(11)$ \\
F13 & $11604(6)$ & $9445(5)$ & $6743.6(5)$ & $234(4)$ \\
F14 & $11665(7)$ & $9026(7)$ & $6646.8(4)$ & $257(4)$ \\
F15 & $12653(4)$ & $9582(4)$ & $6696.9(3)$ & $174(3)$ \\
F16 & $11963(5)$ & $8282(4)$ & $6681.6(3)$ & $193(3)$ \\
F17 & $12100(4)$ & $8806(5)$ & $6789.7(3)$ & $180(3)$ \\
F18 & $11072(5)$ & $8295(5)$ & $6737.7(4)$ & $207(3)$ \\
P4 & 13333 & 6667 & 6667 & $28.8(5)$ \\
F19 & $13361.7(18)$ & $7314.2(17)$ & $6712.1(2)$ & $65.0(9)$ \\
P5 & 3333 & 6667 & $6995.8(2)$ & $53.1(6)$ \\
F20 & 3333 & 6667 & $6914.5(3)$ & $68.3(16)$ \\
F21 & 3333 & 6667 & $7076.7(3)$ & $95(2)$ \\
F22 & $3493(17)$ & $7452(5)$ & $6999.7(5)$ & $203(7)$ \\
F23 & $2667(11)$ & $6795(18)$ & $6992.2(4)$ & $206(8)$ \\
P6 & 10000 & $2547(2)$ & 7500 & $137.2(16)$ \\
F25 & $9802(8)$ & $1905(6)$ & $7452.0(6)$ & $295(6)$ \\
F26 & $9177(6)$ & $2263(11)$ & $7484.0(11)$ & $424(13)$ \\
F27 & $9928(6)$ & $3095(6)$ & $7549.0(5)$ & $221(4)$
\end{tabular}

Table S8: Anisotropic Displacement Parameters $\left(\times 10^{4}\right)$ PL15015_sq. The anisotropic displacement factor exponent takes the form: $-2 \pi^{2}\left[h^{2} a^{* 2} \times U_{11}+\ldots+2 h k a^{*} \times b^{*} \times U_{12}\right]$

\begin{tabular}{|c|c|c|c|c|c|c|}
\hline Atom & $U_{11}$ & $\boldsymbol{U}_{22}$ & $\boldsymbol{U}_{33}$ & $\boldsymbol{U}_{23}$ & $\boldsymbol{U}_{13}$ & $U_{12}$ \\
\hline$\overline{\mathrm{Col}}$ & $24.5(3)$ & $35.9(4)$ & $25.7(3)$ & $0.5(2)$ & $3.5(2)$ & $9.1(3)$ \\
\hline $\mathrm{Co} 2$ & $32.5(4)$ & $32.5(4)$ & $21.3(4)$ & 0 & 0 & $16.26(18)$ \\
\hline N1 & $31.0(18)$ & $46(2)$ & $33.2(16)$ & $2.1(15)$ & $5.0(14)$ & $13.4(16)$ \\
\hline $\mathrm{N} 2$ & $26.1(16)$ & $34.7(18)$ & $28.6(15)$ & $0.1(13)$ & $3.2(13)$ & $10.5(14)$ \\
\hline N3 & $38.0(19)$ & $44(2)$ & $26.4(14)$ & $4.1(14)$ & $1.4(13)$ & $27.4(17)$ \\
\hline N4 & $39.2(19)$ & $50(2)$ & $31.2(15)$ & $2.4(15)$ & $-1.5(14)$ & $32.8(18)$ \\
\hline N5 & $29.5(18)$ & $43(2)$ & $32.4(16)$ & $2.1(15)$ & $1.4(14)$ & $8.6(16)$ \\
\hline N6 & $20.9(15)$ & $41.3(19)$ & $27.3(14)$ & $0.7(14)$ & $0.9(12)$ & $8.9(14)$ \\
\hline N7 & $41.5(19)$ & $34.9(18)$ & $23.1(13)$ & $1.0(13)$ & $0.1(13)$ & $18.9(16)$ \\
\hline N8 & $38.1(19)$ & $36.5(19)$ & $25.8(14)$ & $-2.6(13)$ & $-0.1(13)$ & $17.2(16)$ \\
\hline $\mathrm{C} 1$ & $31(2)$ & $104(5)$ & $42(2)$ & $-2(3)$ & $1(2)$ & $27(3)$ \\
\hline $\mathrm{C} 2$ & $44(3)$ & $106(5)$ & $45(3)$ & $2(3)$ & $11(2)$ & $40(3)$ \\
\hline $\mathrm{C} 3$ & $53(3)$ & $100(5)$ & $37(2)$ & $3(3)$ & $13(2)$ & $48(3)$ \\
\hline $\mathrm{C} 4$ & $52(3)$ & 94(4) & $34(2)$ & $-3(2)$ & $7(2)$ & $44(3)$ \\
\hline $\mathrm{C} 5$ & $37(2)$ & $59(3)$ & $30.1(18)$ & $-0.7(19)$ & $4.7(17)$ & $24(2)$ \\
\hline C6 & $37(2)$ & $66(3)$ & $28.9(19)$ & $-1.0(19)$ & $4.4(17)$ & $30(2)$ \\
\hline $\mathrm{C} 7$ & $58(4)$ & $180(8)$ & $31(2)$ & $-12(3)$ & $-4(2)$ & $71(5)$ \\
\hline $\mathrm{C} 8$ & $55(4)$ & 173(8) & $39(3)$ & $-18(4)$ & $-11(2)$ & $65(5)$ \\
\hline C9 & $30(2)$ & $64(3)$ & $42(2)$ & $1(2)$ & $-0.7(18)$ & $26(2)$ \\
\hline $\mathrm{C} 10$ & $34(2)$ & $38(2)$ & $33.3(18)$ & $2.5(16)$ & $5.5(16)$ & $17.1(18)$ \\
\hline C11 & $33(2)$ & $54(3)$ & $45(2)$ & $-1.8(19)$ & $1.5(18)$ & $25(2)$ \\
\hline C12 & $47(3)$ & $49(3)$ & $122(5)$ & $9(3)$ & $26(3)$ & $25(2)$ \\
\hline C13 & $49(3)$ & $48(3)$ & $112(5)$ & $9(3)$ & $22(3)$ & $29(2)$ \\
\hline $\mathrm{C} 14$ & $33(2)$ & $44(2)$ & $50(2)$ & $-2.4(19)$ & $0.5(18)$ & $19.5(19)$ \\
\hline C15 & $76(4)$ & $61(3)$ & $128(6)$ & $33(4)$ & $46(4)$ & $48(3)$ \\
\hline C16 & $63(3)$ & $71(4)$ & $153(7)$ & $43(4)$ & 49(4) & $48(3)$ \\
\hline C17 & $121(7)$ & $77(5)$ & $357(19)$ & $63(7)$ & $137(10)$ & $60(5)$ \\
\hline C18 & $126(8)$ & $111(6)$ & $460(20)$ & $148(10)$ & $178(12)$ & $92(6)$ \\
\hline C19 & $36(2)$ & $41(2)$ & $35.2(18)$ & $3.2(17)$ & $4.6(16)$ & $24.8(19)$ \\
\hline $\mathrm{C} 20$ & $34(2)$ & $43(2)$ & $43(2)$ & $-0.5(19)$ & $1.5(18)$ & $22(2)$ \\
\hline $\mathrm{C} 21$ & $48(3)$ & $43(3)$ & $58(3)$ & $-3(2)$ & $3(2)$ & $25(2)$ \\
\hline
\end{tabular}




\begin{tabular}{|c|c|c|c|c|c|c|}
\hline Atom & $U_{11}$ & $\boldsymbol{U}_{22}$ & $\boldsymbol{U}_{33}$ & $\boldsymbol{U}_{23}$ & $U_{13}$ & $U_{12}$ \\
\hline $\mathrm{C} 22$ & $49(3)$ & $52(3)$ & $50(2)$ & $-10(2)$ & $2(2)$ & $32(2)$ \\
\hline $\mathrm{C} 23$ & $46(2)$ & $43(2)$ & $30.1(17)$ & $-1.7(17)$ & $-1.9(17)$ & $31(2)$ \\
\hline $\mathrm{C} 24$ & $41(2)$ & $48(2)$ & $28.9(17)$ & $1.3(17)$ & $-0.9(16)$ & $31(2)$ \\
\hline $\mathrm{C} 25$ & $50(3)$ & $67(3)$ & $36(2)$ & $-4(2)$ & $-1.0(19)$ & $40(3)$ \\
\hline $\mathrm{C} 26$ & $65(3)$ & $82(4)$ & $38(2)$ & $-1(2)$ & $8(2)$ & $56(3)$ \\
\hline $\mathrm{C} 27$ & $49(3)$ & $69(3)$ & $46(2)$ & $5(2)$ & $7(2)$ & $40(3)$ \\
\hline C28 & $38(2)$ & $57(3)$ & $40(2)$ & $3(2)$ & $0.9(18)$ & $32(2)$ \\
\hline $\mathrm{C} 29$ & $50(3)$ & $46(3)$ & $43(2)$ & $11(2)$ & $13(2)$ & $14(2)$ \\
\hline $\mathrm{C} 30$ & $71(4)$ & $51(3)$ & $58(3)$ & $17(3)$ & $16(3)$ & $20(3)$ \\
\hline C31 & $95(5)$ & $60(4)$ & $72(4)$ & $27(3)$ & $25(4)$ & $46(4)$ \\
\hline C32 & $69(4)$ & $64(4)$ & $62(3)$ & $13(3)$ & $15(3)$ & $41(3)$ \\
\hline C33 & $41(2)$ & $44(3)$ & $41(2)$ & $2.2(19)$ & $5.0(19)$ & $20(2)$ \\
\hline C34 & $34(2)$ & $44(2)$ & $38(2)$ & $-1.2(18)$ & $2.1(17)$ & $21(2)$ \\
\hline $\mathrm{C} 35$ & $58(3)$ & $61(3)$ & $43(2)$ & $4(2)$ & $16(2)$ & $36(3)$ \\
\hline $\mathrm{C} 36$ & $53(3)$ & $71(3)$ & $39(2)$ & $5(2)$ & $13(2)$ & $38(3)$ \\
\hline C37 & $31(2)$ & $58(3)$ & $31.5(19)$ & $2.2(19)$ & 4.3(16) & $19(2)$ \\
\hline C38 & $27(2)$ & $43(2)$ & $29.7(18)$ & $3.5(17)$ & $2.5(15)$ & $12.1(18)$ \\
\hline C39 & $35(2)$ & $57(3)$ & $30.5(18)$ & $5.0(19)$ & $10.2(17)$ & $23(2)$ \\
\hline $\mathrm{C} 42$ & $37(2)$ & $61(3)$ & $31.3(19)$ & $11(2)$ & $12.0(17)$ & $20(2)$ \\
\hline $\mathrm{C} 40$ & $58(3)$ & $59(3)$ & $26.8(19)$ & $-2(2)$ & $5(2)$ & $18(3)$ \\
\hline $\mathrm{C} 41$ & $61(3)$ & $55(3)$ & $34(2)$ & $0(2)$ & $8(2)$ & $12(3)$ \\
\hline $\mathrm{C} 43$ & $47(3)$ & $74(4)$ & $35(2)$ & $6(2)$ & $2(2)$ & $24(3)$ \\
\hline $\mathrm{C} 44$ & $44(3)$ & $54(3)$ & $41(2)$ & $10(2)$ & $4(2)$ & $13(2)$ \\
\hline C45 & $71(8)$ & $73(8)$ & $56(6)$ & $27(6)$ & $-30(6)$ & $-20(6)$ \\
\hline $\mathrm{C} 40 \mathrm{~A}$ & $58(3)$ & $59(3)$ & $26.8(19)$ & $-2(2)$ & $5(2)$ & $18(3)$ \\
\hline C41A & $61(3)$ & $55(3)$ & $34(2)$ & $0(2)$ & $8(2)$ & $12(3)$ \\
\hline $\mathrm{C} 43 \mathrm{~A}$ & $47(3)$ & $74(4)$ & $35(2)$ & $6(2)$ & $2(2)$ & $24(3)$ \\
\hline $\mathrm{C} 44 \mathrm{~A}$ & $44(3)$ & $54(3)$ & $41(2)$ & $10(2)$ & $4(2)$ & $13(2)$ \\
\hline $\mathrm{C} 45 \mathrm{~A}$ & $99(12)$ & $97(12)$ & $48(7)$ & $9(7)$ & $-33(8)$ & $2(10)$ \\
\hline C46 & 101(10) & $55(7)$ & $36(4)$ & $8(4)$ & $-8(5)$ & $-19(6)$ \\
\hline C46A & 178(19) & $170(20)$ & $39(6)$ & $34(9)$ & $-39(10)$ & $-73(17)$ \\
\hline C47 & $47(2)$ & $45(2)$ & $24.3(16)$ & $5.4(16)$ & $4.3(17)$ & $23(2)$ \\
\hline C48 & $43(2)$ & $54(3)$ & $29.4(18)$ & $5.0(19)$ & $9.3(18)$ & $23(2)$ \\
\hline C49 & $45(3)$ & $71(3)$ & $39(2)$ & $8(2)$ & $12(2)$ & $30(3)$ \\
\hline $\mathrm{C} 50$ & $41(3)$ & $65(3)$ & $41(2)$ & $10(2)$ & 8.0(19) & $29(2)$ \\
\hline C51 & $41(2)$ & $48(2)$ & $26.0(16)$ & $-0.4(17)$ & $-1.4(16)$ & $23(2)$ \\
\hline C52 & $40(2)$ & $45(2)$ & $32.8(18)$ & $3.3(18)$ & $-0.2(17)$ & $24(2)$ \\
\hline C53 & $49(3)$ & $82(4)$ & $60(3)$ & $26(3)$ & $10(2)$ & $43(3)$ \\
\hline $\mathrm{C} 54$ & $71(4)$ & $104(5)$ & $95(5)$ & $58(4)$ & $18(4)$ & $58(4)$ \\
\hline C55 & $52(3)$ & $76(4)$ & $52(3)$ & $24(3)$ & $4(2)$ & $34(3)$ \\
\hline C56 & $46(3)$ & $45(2)$ & $31.6(18)$ & $7.9(18)$ & $2.3(18)$ & $22(2)$ \\
\hline $\mathrm{P} 1$ & $44.4(7)$ & $64.3(9)$ & $54.1(7)$ & $23.6(6)$ & $11.8(6)$ & $26.1(7)$ \\
\hline F1 & $92(3)$ & $69(2)$ & $119(3)$ & $14(2)$ & $14(3)$ & $41(2)$ \\
\hline $\mathrm{F} 2$ & $47.4(18)$ & $87(3)$ & $97(3)$ & $46(2)$ & $23.1(18)$ & $32.8(18)$ \\
\hline F3 & $54(2)$ & $130(3)$ & $67(2)$ & $50(2)$ & 14.1(16) & $41(2)$ \\
\hline F4 & $82(3)$ & 104(3) & $103(3)$ & $-24(3)$ & $-18(2)$ & $52(3)$ \\
\hline F5 & $48.6(18)$ & $95(3)$ & $76(2)$ & $28(2)$ & 7.2(16) & $27.6(19)$ \\
\hline F6 & $78(2)$ & $86(2)$ & $60.1(18)$ & $29.5(18)$ & $11.6(17)$ & $46(2)$ \\
\hline P2 & $60.6(12)$ & $101.9(17)$ & $207(3)$ & $48.9(18)$ & $4.4(15)$ & $43.6(12)$ \\
\hline F7 & $108(4)$ & $152(5)$ & $177(5)$ & $37(4)$ & $16(4)$ & $89(4)$ \\
\hline F8 & $151(5)$ & 197(7) & $257(7)$ & $-18(5)$ & $-68(5)$ & $127(5)$ \\
\hline F9 & 97(4) & $173(5)$ & $212(6)$ & $60(5)$ & $27(4)$ & 77(4) \\
\hline F10 & $147(5)$ & $174(6)$ & $290(8)$ & $-16(5)$ & $-51(5)$ & $109(5)$ \\
\hline F11 & $146(5)$ & $266(8)$ & $170(5)$ & $52(5)$ & $20(4)$ & $159(5)$ \\
\hline F12 & $104(4)$ & $199(6)$ & $358(9)$ & $126(6)$ & $56(5)$ & 93(4) \\
\hline P3 & $196(3)$ & $102.5(19)$ & $83.3(14)$ & 27.1(14) & $50.1(18)$ & $60(2)$ \\
\hline F13 & $285(10)$ & $216(7)$ & $247(8)$ & $92(6)$ & $128(7)$ & $159(7)$ \\
\hline F14 & 291(10) & $273(10)$ & $163(6)$ & $96(6)$ & $36(6)$ & 107(8) \\
\hline F15 & $218(6)$ & $151(5)$ & $114(4)$ & $25(4)$ & $88(4)$ & $62(5)$ \\
\hline F16 & $300(9)$ & $142(5)$ & $127(4)$ & $36(4)$ & $100(5)$ & $104(5)$ \\
\hline F17 & $170(5)$ & $306(9)$ & $84(3)$ & $55(4)$ & $64(3)$ & $134(6)$ \\
\hline F18 & $199(6)$ & $237(7)$ & $154(6)$ & $80(5)$ & $41(5)$ & $86(6)$ \\
\hline P4 & $28.1(7)$ & $28.1(7)$ & $30.1(10)$ & 0 & 0 & $14.1(4)$ \\
\hline F19 & $63(2)$ & $51.3(18)$ & $80(2)$ & $-27.8(16)$ & $3.1(16)$ & $28.1(16)$ \\
\hline
\end{tabular}




\begin{tabular}{llccccc}
\hline Atom & $\boldsymbol{U}_{\mathbf{1 1}}$ & $\boldsymbol{U}_{\mathbf{2 2}}$ & $\boldsymbol{U}_{\mathbf{3 3}}$ & $\boldsymbol{U}_{\mathbf{2 3}}$ & $\boldsymbol{U}_{\mathbf{1 3}}$ & $\boldsymbol{U}_{\mathbf{1 2}}$ \\
\hline P5 & $56.2(8)$ & $56.2(8)$ & $47.0(11)$ & 0 & 0 & $28.1(4)$ \\
F20 & $78(2)$ & $78(2)$ & $49(3)$ & 0 & 0 & $39.1(12)$ \\
F21 & $118(4)$ & $118(4)$ & $49(3)$ & 0 & 0 & $59.2(19)$ \\
F22 & $430(20)$ & $48(4)$ & $95(6)$ & $-3(4)$ & $-6(12)$ & $96(8)$ \\
F23 & $192(11)$ & $490(20)$ & $73(5)$ & $77(12)$ & $49(7)$ & $274(16)$ \\
P6 & $146(4)$ & $118(2)$ & $157(4)$ & $-25.0(16)$ & $-50(3)$ & $72.9(19)$ \\
F25 & $338(14)$ & $242(10)$ & $320(13)$ & $-148(10)$ & $-43(11)$ & $156(10)$ \\
F26 & $205(9)$ & $480(20)$ & $610(30)$ & $-310(20)$ & $-143(12)$ & $194(11)$ \\
F27 & $208(8)$ & $261(10)$ & $256(9)$ & $-98(8)$ & $-73(8)$ & $164(8)$
\end{tabular}

Table S9: Bond Lengths in $\AA$ for PL15015_sq.

\begin{tabular}{|c|c|c|c|c|c|}
\hline Atom & Atom & Length/Å & Atom & Atom & Length/Å \\
\hline$\overline{\text { Co1 }}$ & N1 & $1.925(4)$ & $\overline{\mathrm{C} 20}$ & $\mathrm{C} 21$ & $1.402(6)$ \\
\hline Co1 & $\mathrm{N} 2$ & $1.925(3)$ & $\mathrm{C} 21$ & $\mathrm{C} 22$ & $1.376(7)$ \\
\hline Col & $\mathrm{N} 3^{1}$ & $1.926(4)$ & $\mathrm{C} 22$ & $\mathrm{C} 23$ & $1.385(7)$ \\
\hline Col & $\mathrm{N} 4^{1}$ & $1.938(4)$ & $\mathrm{C} 23$ & $\mathrm{C} 24$ & $1.471(6)$ \\
\hline Col & N5 & $1.934(4)$ & $\mathrm{C} 24$ & $\mathrm{C} 25$ & $1.392(6)$ \\
\hline Co1 & N6 & $1.948(3)$ & $\mathrm{C} 25$ & $\mathrm{C} 26$ & $1.372(7)$ \\
\hline $\mathrm{Co} 2$ & $\mathrm{~N} 7^{1}$ & $1.926(4)$ & $\mathrm{C} 26$ & $\mathrm{C} 27$ & $1.376(8)$ \\
\hline $\mathrm{Co} 2$ & N7 & $1.926(4)$ & $\mathrm{C} 27$ & $\mathrm{C} 28$ & $1.400(6)$ \\
\hline $\mathrm{Co} 2$ & $\mathrm{~N}^{2}{ }^{2}$ & $1.926(4)$ & $\mathrm{C} 29$ & $\mathrm{C} 30$ & $1.366(8)$ \\
\hline $\mathrm{Co} 2$ & $N 8^{2}$ & $1.935(4)$ & $\mathrm{C} 30$ & C31 & $1.383(9)$ \\
\hline $\mathrm{Co} 2$ & N8 & $1.935(4)$ & C31 & $\mathrm{C} 32$ & $1.397(8)$ \\
\hline $\mathrm{Co} 2$ & N8 ${ }^{1}$ & $1.935(4)$ & C32 & $\mathrm{C} 33$ & $1.384(7)$ \\
\hline N1 & $\mathrm{C} 1$ & $1.340(6)$ & C33 & C34 & $1.450(6)$ \\
\hline N1 & $\mathrm{C} 5$ & $1.351(6)$ & C34 & $\mathrm{C} 35$ & $1.406(6)$ \\
\hline N2 & C6 & $1.364(5)$ & $\mathrm{C} 35$ & $\mathrm{C} 36$ & $1.385(7)$ \\
\hline N2 & $\mathrm{C} 10$ & $1.347(5)$ & C36 & C37 & $1.385(7)$ \\
\hline N3 & $\mathrm{Col}^{2}$ & $1.926(4)$ & C37 & $\mathrm{C} 38$ & $1.394(6)$ \\
\hline N3 & C19 & $1.358(5)$ & C37 & C39 & $1.478(6)$ \\
\hline N3 & $\mathrm{C} 23$ & $1.357(5)$ & C39 & $\mathrm{C} 40$ & $1.380(7)$ \\
\hline N4 & $\mathrm{Co}^{2}$ & $1.938(3)$ & C39 & C44 & $1.401(6)$ \\
\hline N4 & $\mathrm{C} 24$ & $1.362(6)$ & C39 & $\mathrm{C} 40 \mathrm{~A}$ & $1.380(7)$ \\
\hline N4 & $\mathrm{C} 28$ & $1.347(6)$ & C39 & $\mathrm{C} 44 \mathrm{~A}$ & $1.401(6)$ \\
\hline N5 & $\mathrm{C} 29$ & $1.345(6)$ & $\mathrm{C} 42$ & C41 & $1.375(7)$ \\
\hline N5 & $\mathrm{C} 33$ & $1.355(6)$ & $\mathrm{C} 42$ & $\mathrm{C} 43$ & $1.400(8)$ \\
\hline N6 & $\mathrm{C} 34$ & $1.343(6)$ & $\mathrm{C} 42$ & C41A & $1.375(7)$ \\
\hline N6 & C38 & $1.342(5)$ & $\mathrm{C} 42$ & $\mathrm{C} 43 \mathrm{~A}$ & $1.400(8)$ \\
\hline N7 & $\mathrm{C} 47$ & $1.360(5)$ & $\mathrm{C} 42$ & $\mathrm{C} 48$ & $1.485(6)$ \\
\hline N7 & C51 & $1.348(5)$ & $\mathrm{C} 40$ & $\mathrm{C} 41$ & $1.389(7)$ \\
\hline N8 & $\mathrm{C} 52$ & $1.347(6)$ & $\mathrm{C} 41$ & $\mathrm{C} 46$ & $1.590(12)$ \\
\hline N8 & C56 & $1.346(5)$ & $\mathrm{C} 43$ & $\mathrm{C} 44$ & $1.384(7)$ \\
\hline $\mathrm{C} 1$ & $\mathrm{C} 2$ & $1.381(7)$ & C44 & $\mathrm{C} 45$ & $1.516(12)$ \\
\hline $\mathrm{C} 2$ & C3 & $1.377(7)$ & $\mathrm{C} 40 \mathrm{~A}$ & C41A & $1.389(7)$ \\
\hline $\mathrm{C} 3$ & $\mathrm{C} 4$ & $1.401(7)$ & $\mathrm{C} 40 \mathrm{~A}$ & C46A & $1.514(16)$ \\
\hline $\mathrm{C} 4$ & $\mathrm{C} 5$ & $1.379(6)$ & $\mathrm{C} 43 \mathrm{~A}$ & C44A & $1.384(7)$ \\
\hline C5 & C6 & $1.466(6)$ & $\mathrm{C} 43 \mathrm{~A}$ & $\mathrm{C} 45 \mathrm{~A}$ & $1.563(13)$ \\
\hline C6 & $\mathrm{C} 7$ & $1.363(7)$ & C47 & C48 & $1.402(6)$ \\
\hline $\mathrm{C} 7$ & $\mathrm{C} 8$ & $1.389(8)$ & $\mathrm{C} 48$ & $\mathrm{C} 49$ & $1.371(7)$ \\
\hline $\mathrm{C} 8$ & C9 & $1.379(7)$ & $\mathrm{C} 49$ & $\mathrm{C} 50$ & $1.406(7)$ \\
\hline C9 & $\mathrm{C} 10$ & $1.381(6)$ & $\mathrm{C} 50$ & C51 & $1.381(6)$ \\
\hline C9 & $\mathrm{C} 11$ & $1.501(6)$ & C51 & C52 & $1.470(6)$ \\
\hline $\mathrm{C} 11$ & $\mathrm{C} 12$ & $1.350(7)$ & C52 & C53 & $1.379(6)$ \\
\hline C11 & $\mathrm{C} 16$ & $1.370(8)$ & $\mathrm{C} 53$ & C54 & $1.387(8)$ \\
\hline $\mathrm{C} 12$ & $\mathrm{C} 13$ & $1.397(8)$ & C54 & C55 & $1.371(8)$ \\
\hline $\mathrm{C} 12$ & $\mathrm{C} 17$ & $1.519(10)$ & $\mathrm{C} 55$ & C56 & $1.384(7)$ \\
\hline C13 & $\mathrm{C} 14$ & $1.364(8)$ & $\mathrm{P} 1$ & $\mathrm{~F} 1$ & $1.574(4)$ \\
\hline C14 & $\mathrm{C} 15$ & $1.390(7)$ & $\mathrm{P} 1$ & $\mathrm{~F} 2$ & $1.612(3)$ \\
\hline $\mathrm{C} 14$ & $\mathrm{C} 20$ & $1.482(6)$ & $\mathrm{P} 1$ & F3 & $1.585(3)$ \\
\hline C15 & $\mathrm{C} 16$ & $1.409(8)$ & $\mathrm{P} 1$ & $\mathrm{~F} 4$ & $1.585(4)$ \\
\hline $\mathrm{C} 15$ & $\mathrm{C} 18$ & $1.467(11)$ & $\mathrm{P} 1$ & F5 & $1.580(3)$ \\
\hline C19 & $\mathrm{C} 20$ & $1.391(6)$ & $\mathrm{P} 1$ & F6 & $1.592(3)$ \\
\hline
\end{tabular}




\begin{tabular}{lll}
\hline Atom & Atom & Length/A \\
\hline P2 & F7 & $1.570(6)$ \\
P2 & F8 & $1.550(7)$ \\
P2 & F9 & $1.562(6)$ \\
P2 & F10 & $1.586(7)$ \\
P2 & F11 & $1.568(7)$ \\
P2 & F12 & $1.532(7)$ \\
P3 & F13 & $1.580(8)$ \\
P3 & F14 & $1.556(8)$ \\
P3 & F15 & $1.532(7)$ \\
P3 & F16 & $1.548(7)$ \\
P3 & F17 & $1.479(6)$ \\
P3 & F18 & $1.583(8)$ \\
P4 & F19 & $1.579(3)$ \\
P4 & F19 & $1.579(3)$ \\
P4 & F19 & $1.579(3)$ \\
P4 & F19 & $1.579(3)$ \\
P4 & F19 & $1.579(3)$ \\
P4 & F19 & $1.579(3)$ \\
P5 & F20 & $1.586(5)$ \\
P5 & F21 & $1.579(6)$
\end{tabular}

\begin{tabular}{lll}
\hline Atom & Atom & Length/A \\
\hline P5 & F22 & $1.484(7)$ \\
P5 & F22 & $1.484(8)$ \\
P5 & F22 & $1.484(8)$ \\
P5 & F23 & $1.523(7)$ \\
P5 & F23 $^{8}$ & $1.523(8)$ \\
P5 & F23 $^{9}$ & $1.523(7)$ \\
F22 & F23 $^{8}$ & $1.455(17)$ \\
F22 & F23 & $1.563(16)$ \\
F23 & F22 & $1.455(17)$ \\
P6 & F25 & $1.503(8)$ \\
P6 & F25 & $1.503(8)$ \\
P6 & F26 & $1.523(10)$ \\
P6 & F26 & $1.523(10)$ \\
P6 & F27 & $1.542(8)$ \\
P6 & F27 & $1.542(8)$ \\
$------• ~$ & &
\end{tabular}

${ }^{1} 1+\mathrm{Y}-\mathrm{X}, 1-\mathrm{X},+\mathrm{Z} ; \quad{ }^{2} 1-\mathrm{Y},+\mathrm{X}-\mathrm{Y},+\mathrm{Z} ; \quad{ }^{3} 2 / 3-\mathrm{Y}+\mathrm{X},-2 / 3+\mathrm{X}, 4 / 3-\mathrm{Z}$ ${ }^{4} 8 / 3-X, 4 / 3-Y, 4 / 3-Z ; \quad{ }^{5} 2-Y,+X-Y,+Z ; \quad{ }^{6} 2+Y-X, 2-X,+Z$ ${ }^{7} 2 / 3+\mathrm{Y}, 4 / 3-\mathrm{X}+\mathrm{Y}, 4 / 3-\mathrm{Z} ;{ }^{8}+\mathrm{Y}-\mathrm{X}, 1-\mathrm{X},+\mathrm{Z} ;{ }^{9} 1-\mathrm{Y}, 1+\mathrm{X}-\mathrm{Y},+\mathrm{Z} ;{ }^{10} 2$ $\mathrm{X}, 1-\mathrm{X}+\mathrm{Y}, 3 / 2-\mathrm{Z}$

Table S10: Bond Angles in ${ }^{\circ}$ for PL15015_sq.

\begin{tabular}{|c|c|c|c|}
\hline Atom & Atom & Atom & Angle $/^{\circ}$ \\
\hline N1 & Co1 & N2 & $83.22(15)$ \\
\hline N1 & Col & $\mathrm{N} 3^{1}$ & $88.22(15)$ \\
\hline N1 & Col & $\mathrm{N} 4^{1}$ & $96.62(15)$ \\
\hline N1 & Col & N5 & $94.33(16)$ \\
\hline N1 & Col & N6 & $176.99(16)$ \\
\hline N2 & Col & $\mathrm{N} 3^{1}$ & $95.33(15)$ \\
\hline $\mathrm{N} 2$ & Col & $\mathrm{N} 4^{1}$ & $178.79(17)$ \\
\hline N2 & Col & N5 & $85.96(15)$ \\
\hline N2 & Col & N6 & $94.92(14)$ \\
\hline $\mathrm{N} 3^{1}$ & $\mathrm{Co} 1$ & $\mathrm{~N} 4^{1}$ & $83.47(15)$ \\
\hline $\mathrm{N} 3^{1}$ & Col & N5 & $177.27(14)$ \\
\hline $\mathrm{N} 3^{1}$ & $\mathrm{Co} 1$ & N6 & $94.33(15)$ \\
\hline $\mathrm{N} 4^{1}$ & Col & N6 & $85.29(14)$ \\
\hline N5 & Col & $\mathrm{N} 4^{1}$ & $95.25(16)$ \\
\hline N5 & $\mathrm{Co} 1$ & N6 & $83.15(15)$ \\
\hline $\mathrm{N} 7^{1}$ & $\mathrm{Co} 2$ & $\mathrm{~N} 7^{2}$ & $96.08(13)$ \\
\hline $\mathrm{N} 7^{1}$ & $\mathrm{Co} 2$ & N7 & $96.07(13)$ \\
\hline N7 & $\mathrm{Co} 2$ & $\mathrm{~N}^{2}{ }^{2}$ & $96.07(13)$ \\
\hline $\mathrm{N} 7^{2}$ & $\mathrm{Co} 2$ & N8 & $177.03(17)$ \\
\hline$N 7^{2}$ & $\mathrm{Co} 2$ & $N 8^{1}$ & $86.82(14)$ \\
\hline N7 & $\mathrm{Co} 2$ & N8 & $82.91(14)$ \\
\hline N7 & $\mathrm{Co} 2$ & $N 8^{2}$ & $86.82(15)$ \\
\hline $\mathrm{N} 7^{1}$ & $\mathrm{Co} 2$ & N8 & $86.82(15)$ \\
\hline $\mathrm{N} 7^{2}$ & $\mathrm{Co} 2$ & $N 8^{2}$ & $82.91(15)$ \\
\hline $\mathrm{N} 7^{1}$ & $\mathrm{Co} 2$ & $N 8^{1}$ & $82.91(15)$ \\
\hline $\mathrm{N} 7^{1}$ & $\mathrm{Co} 2$ & $N 8^{2}$ & $177.03(15)$ \\
\hline N7 & $\mathrm{Co} 2$ & $N 8^{1}$ & $177.03(15)$ \\
\hline$N 8^{2}$ & $\mathrm{Co} 2$ & N8 & $94.23(13)$ \\
\hline$N 8^{2}$ & $\mathrm{Co} 2$ & $N 8^{1}$ & $94.24(13)$ \\
\hline N8 ${ }^{1}$ & $\mathrm{Co} 2$ & N8 & $94.24(13)$ \\
\hline $\mathrm{C} 1$ & N1 & Co1 & $126.4(3)$ \\
\hline $\mathrm{C} 1$ & N1 & C5 & $118.7(4)$ \\
\hline $\mathrm{C} 5$ & N1 & Col & $114.9(3)$ \\
\hline $\mathrm{C} 6$ & N2 & Co1 & $114.2(3)$ \\
\hline $\mathrm{C} 10$ & N2 & $\mathrm{Co} 1$ & $126.7(3)$ \\
\hline $\mathrm{C} 10$ & $\mathrm{~N} 2$ & C6 & $118.8(4)$ \\
\hline C19 & N3 & $\mathrm{Co}^{2}$ & $126.9(3)$ \\
\hline $\mathrm{C} 23$ & N3 & $\mathrm{Co}^{2}$ & $114.6(3)$ \\
\hline
\end{tabular}

\begin{tabular}{llll}
\hline Atom & Atom & Atom & Angle ${ }^{\circ}$ \\
\hline C23 & N3 & C19 & $118.4(4)$ \\
C24 & N4 & Co1 ${ }^{2}$ & $113.7(3)$ \\
C28 & N4 & Co1 ${ }^{2}$ & $126.1(3)$ \\
C28 & N4 & C24 & $119.3(4)$ \\
C29 & N5 & Co1 & $127.3(4)$ \\
C29 & N5 & C33 & $118.7(4)$ \\
C33 & N5 & Co1 & $113.8(3)$ \\
C34 & N6 & Co1 & $113.4(3)$ \\
C38 & N6 & Co1 & $126.6(3)$ \\
C38 & N6 & C34 & $119.3(4)$ \\
C47 & N7 & Co2 & $126.6(3)$ \\
C51 & N7 & Co2 & $114.7(3)$ \\
C51 & N7 & C47 & $118.4(4)$ \\
C52 & N8 & Co2 & $114.4(3)$ \\
C56 & N8 & Co2 & $126.0(3)$ \\
C56 & N8 & C52 & $119.5(4)$ \\
N1 & C1 & C2 & $121.9(5)$ \\
C3 & C2 & C1 & $119.7(5)$ \\
C2 & C3 & C4 & $118.6(4)$ \\
C5 & C4 & C3 & $118.5(5)$ \\
N1 & C5 & C4 & $122.4(4)$ \\
N1 & C5 & C6 & $113.6(4)$ \\
C4 & C5 & C6 & $123.9(4)$ \\
N2 & C6 & C5 & $113.6(4)$ \\
C7 & C6 & N2 & $121.0(4)$ \\
C7 & C6 & C5 & $125.4(4)$ \\
C6 & C7 & C8 & $119.8(5)$ \\
C9 & C8 & C7 & $119.7(5)$ \\
C8 & C9 & C10 & $118.0(4)$ \\
C8 & C9 & C11 & $123.6(4)$ \\
C10 & C9 & C11 & $118.4(4)$ \\
N2 & C10 & C9 & $122.6(4)$ \\
C12 & C11 & C9 & $121.4(5)$ \\
C12 & C11 & C16 & $120.3(5)$ \\
C16 & C11 & C9 & $118.3(5)$ \\
C11 & C12 & C13 & $117.0(5)$ \\
C13 & C12 & C17 & $121.5(6)$ \\
& C12 & C17 & $121.2(6)$ \\
& & &
\end{tabular}




\begin{tabular}{|c|c|c|c|}
\hline Atom & Atom & Atom & Angle $/^{\circ}$ \\
\hline C14 & C13 & C12 & $124.3(5)$ \\
\hline C13 & C14 & $\mathrm{C} 15$ & $118.6(5)$ \\
\hline C13 & $\mathrm{C} 14$ & $\mathrm{C} 20$ & $118.7(4)$ \\
\hline $\mathrm{C} 15$ & C14 & $\mathrm{C} 20$ & $122.6(5)$ \\
\hline $\mathrm{C} 14$ & $\mathrm{C} 15$ & $\mathrm{C} 16$ & $116.7(6)$ \\
\hline $\mathrm{C} 14$ & $\mathrm{C} 15$ & C18 & $124.5(6)$ \\
\hline $\mathrm{C} 16$ & $\mathrm{C} 15$ & C18 & $118.7(6)$ \\
\hline C11 & C16 & $\mathrm{C} 15$ & $122.9(6)$ \\
\hline N3 & C19 & $\mathrm{C} 20$ & $122.9(4)$ \\
\hline C19 & $\mathrm{C} 20$ & C14 & $121.7(4)$ \\
\hline C19 & $\mathrm{C} 20$ & $\mathrm{C} 21$ & $117.6(4)$ \\
\hline $\mathrm{C} 21$ & $\mathrm{C} 20$ & $\mathrm{C} 14$ & $120.7(4)$ \\
\hline $\mathrm{C} 22$ & $\mathrm{C} 21$ & $\mathrm{C} 20$ & $119.6(5)$ \\
\hline $\mathrm{C} 21$ & $\mathrm{C} 22$ & $\mathrm{C} 23$ & $119.9(4)$ \\
\hline N3 & $\mathrm{C} 23$ & $\mathrm{C} 22$ & $121.5(4)$ \\
\hline N3 & $\mathrm{C} 23$ & $\mathrm{C} 24$ & $113.7(4)$ \\
\hline $\mathrm{C} 22$ & $\mathrm{C} 23$ & $\mathrm{C} 24$ & $124.8(4)$ \\
\hline N4 & $\mathrm{C} 24$ & $\mathrm{C} 23$ & $113.8(4)$ \\
\hline N4 & $\mathrm{C} 24$ & $\mathrm{C} 25$ & $121.8(4)$ \\
\hline $\mathrm{C} 25$ & $\mathrm{C} 24$ & $\mathrm{C} 23$ & $124.4(4)$ \\
\hline $\mathrm{C} 26$ & $\mathrm{C} 25$ & $\mathrm{C} 24$ & $118.2(5)$ \\
\hline $\mathrm{C} 25$ & $\mathrm{C} 26$ & $\mathrm{C} 27$ & $120.7(4)$ \\
\hline $\mathrm{C} 26$ & $\mathrm{C} 27$ & $\mathrm{C} 28$ & $118.9(5)$ \\
\hline N4 & $\mathrm{C} 28$ & $\mathrm{C} 27$ & $120.9(5)$ \\
\hline N5 & $\mathrm{C} 29$ & C30 & $122.8(5)$ \\
\hline $\mathrm{C} 29$ & $\mathrm{C} 30$ & C31 & $119.2(5)$ \\
\hline C30 & $\mathrm{C} 31$ & $\mathrm{C} 32$ & $118.8(6)$ \\
\hline C33 & $\mathrm{C} 32$ & C31 & $119.1(5)$ \\
\hline N5 & C33 & C 32 & $121.4(4)$ \\
\hline N5 & $\mathrm{C} 33$ & C34 & $114.1(4)$ \\
\hline C32 & $\mathrm{C} 33$ & C34 & $124.4(4)$ \\
\hline N6 & C34 & C33 & $114.8(4)$ \\
\hline N6 & C34 & C35 & $121.4(4)$ \\
\hline $\mathrm{C} 35$ & C34 & C33 & $123.7(4)$ \\
\hline C36 & $\mathrm{C} 35$ & C34 & $118.3(5)$ \\
\hline $\mathrm{C} 35$ & $\mathrm{C} 36$ & C37 & $120.6(4)$ \\
\hline C36 & C37 & C38 & $117.4(4)$ \\
\hline C36 & C37 & C39 & $122.6(4)$ \\
\hline C38 & C37 & C39 & $120.0(5)$ \\
\hline N6 & C38 & C37 & $122.9(4)$ \\
\hline $\mathrm{C} 40$ & C39 & C37 & $122.0(4)$ \\
\hline $\mathrm{C} 40$ & C39 & $\mathrm{C} 44$ & $119.5(4)$ \\
\hline C44 & C39 & C37 & $118.5(5)$ \\
\hline C40A & C39 & C37 & $122.0(4)$ \\
\hline C40A & C39 & C44A & $119.5(4)$ \\
\hline C44A & C39 & C37 & $118.5(5)$ \\
\hline C41 & $\mathrm{C} 42$ & $\mathrm{C} 43$ & $119.2(4)$ \\
\hline $\mathrm{C} 41$ & $\mathrm{C} 42$ & $\mathrm{C} 48$ & $121.7(5)$ \\
\hline C43 & $\mathrm{C} 42$ & C48 & $119.1(4)$ \\
\hline C41A & $\mathrm{C} 42$ & C43A & $119.2(4)$ \\
\hline $\mathrm{C} 41 \mathrm{~A}$ & $\mathrm{C} 42$ & C48 & $121.7(5)$ \\
\hline $\mathrm{C} 43 \mathrm{~A}$ & $\mathrm{C} 42$ & $\mathrm{C} 48$ & $119.1(4)$ \\
\hline C39 & $\mathrm{C} 40$ & C41 & $119.9(4)$ \\
\hline $\mathrm{C} 42$ & C41 & $\mathrm{C} 40$ & $121.2(5)$ \\
\hline $\mathrm{C} 42$ & $\mathrm{C} 41$ & $\mathrm{C} 46$ & $125.7(6)$ \\
\hline $\mathrm{C} 40$ & $\mathrm{C} 41$ & $\mathrm{C} 46$ & $113.1(5)$ \\
\hline $\mathrm{C} 44$ & $\mathrm{C} 43$ & $\mathrm{C} 42$ & $120.0(5)$ \\
\hline C39 & $\mathrm{C} 44$ & $\mathrm{C} 45$ & $124.4(6)$ \\
\hline $\mathrm{C} 43$ & $\mathrm{C} 44$ & C39 & $120.2(5)$ \\
\hline $\mathrm{C} 43$ & C44 & C45 & $115.1(6)$ \\
\hline C39 & $\mathrm{C} 40 \mathrm{~A}$ & C41A & $119.9(4)$ \\
\hline C39 & C40A & C46A & $126.0(9)$ \\
\hline C41A & $\mathrm{C} 40 \mathrm{~A}$ & C46A & $113.9(9)$ \\
\hline $\mathrm{C} 42$ & C41A & $\mathrm{C} 40 \mathrm{~A}$ & $121.2(5)$ \\
\hline
\end{tabular}

\begin{tabular}{|c|c|c|c|}
\hline Atom & Atom & Atom & Angle $/^{\circ}$ \\
\hline$\overline{\mathrm{C} 42}$ & C43A & $\mathrm{C} 45 \mathrm{~A}$ & $124.8(8)$ \\
\hline C44A & $\mathrm{C} 43 \mathrm{~A}$ & $\mathrm{C} 42$ & $120.0(5)$ \\
\hline C44A & $\mathrm{C} 43 \mathrm{~A}$ & $\mathrm{C} 45 \mathrm{~A}$ & $115.1(8)$ \\
\hline $\mathrm{C} 43 \mathrm{~A}$ & C44A & C39 & $120.2(5)$ \\
\hline N7 & C47 & $\mathrm{C} 48$ & $122.0(4)$ \\
\hline $\mathrm{C} 47$ & $\mathrm{C} 48$ & $\mathrm{C} 42$ & $119.2(4)$ \\
\hline C49 & $\mathrm{C} 48$ & $\mathrm{C} 42$ & $122.0(4)$ \\
\hline C49 & $\mathrm{C} 48$ & $\mathrm{C} 47$ & $118.8(4)$ \\
\hline $\mathrm{C} 48$ & C49 & $\mathrm{C} 50$ & $119.7(5)$ \\
\hline C51 & C50 & $\mathrm{C} 49$ & $118.3(5)$ \\
\hline N7 & C51 & $\mathrm{C} 50$ & $122.8(4)$ \\
\hline N7 & C51 & C52 & $113.5(4)$ \\
\hline $\mathrm{C} 50$ & C51 & $\mathrm{C} 52$ & $123.7(4)$ \\
\hline N8 & C52 & C51 & $113.8(4)$ \\
\hline N8 & C52 & C53 & $121.5(4)$ \\
\hline C53 & C52 & C51 & $124.7(4)$ \\
\hline C52 & C53 & C54 & $118.7(5)$ \\
\hline C55 & C54 & $\mathrm{C} 53$ & $120.0(5)$ \\
\hline C54 & C55 & C56 & $118.8(5)$ \\
\hline N8 & C56 & C55 & $121.6(5)$ \\
\hline $\mathrm{F} 1$ & $\mathrm{P} 1$ & $\mathrm{~F} 2$ & $89.0(2)$ \\
\hline $\mathrm{F} 1$ & $\mathrm{P} 1$ & F3 & $90.3(3)$ \\
\hline F1 & $\mathrm{P} 1$ & $\mathrm{~F} 4$ & $179.0(3)$ \\
\hline F1 & $\mathrm{P} 1$ & F5 & $90.6(3)$ \\
\hline F1 & $\mathrm{P} 1$ & F6 & $90.7(2)$ \\
\hline F3 & $\mathrm{P} 1$ & $\mathrm{~F} 2$ & $89.06(19)$ \\
\hline F3 & $\mathrm{P} 1$ & F4 & $90.5(3)$ \\
\hline F3 & $\mathrm{P} 1$ & F6 & $178.5(3)$ \\
\hline F4 & $\mathrm{P} 1$ & $\mathrm{~F} 2$ & $90.4(2)$ \\
\hline F4 & $\mathrm{P} 1$ & F6 & $88.6(2)$ \\
\hline F5 & $\mathrm{P} 1$ & $\mathrm{~F} 2$ & $179.5(3)$ \\
\hline F5 & $\mathrm{P} 1$ & F3 & $90.73(19)$ \\
\hline F5 & $\mathrm{P} 1$ & $\mathrm{~F} 4$ & $90.1(2)$ \\
\hline F5 & $\mathrm{P} 1$ & F6 & $90.4(2)$ \\
\hline F6 & $\mathrm{P} 1$ & $\mathrm{~F} 2$ & $89.8(2)$ \\
\hline F7 & $\mathrm{P} 2$ & F10 & $176.9(5)$ \\
\hline F8 & $\mathrm{P} 2$ & F7 & $88.3(4)$ \\
\hline F8 & $\mathrm{P} 2$ & F9 & $93.1(5)$ \\
\hline F8 & $\mathrm{P} 2$ & F10 & $89.6(4)$ \\
\hline F8 & P2 & F11 & $176.2(5)$ \\
\hline F9 & $\mathrm{P} 2$ & F7 & $91.4(4)$ \\
\hline F9 & $\mathrm{P} 2$ & F10 & $86.4(4)$ \\
\hline F9 & $\mathrm{P} 2$ & F11 & $89.0(4)$ \\
\hline F11 & P2 & F7 & $94.8(4)$ \\
\hline F11 & $\mathrm{P} 2$ & F10 & $87.4(4)$ \\
\hline F12 & $\mathrm{P} 2$ & F7 & $89.7(4)$ \\
\hline F12 & $\mathrm{P} 2$ & F8 & $89.2(5)$ \\
\hline F12 & $\mathrm{P} 2$ & F9 & $177.5(5)$ \\
\hline F12 & $\mathrm{P} 2$ & F10 & $92.6(5)$ \\
\hline F12 & $\mathrm{P} 2$ & F11 & $88.6(5)$ \\
\hline F13 & P3 & F18 & $81.1(6)$ \\
\hline F14 & P3 & F13 & $84.5(6)$ \\
\hline F14 & P3 & F18 & $91.7(6)$ \\
\hline F15 & P3 & F13 & $91.6(5)$ \\
\hline F15 & P3 & F14 & $81.7(5)$ \\
\hline F15 & P3 & F16 & $98.8(5)$ \\
\hline F15 & P3 & F18 & $170.6(5)$ \\
\hline F16 & P3 & F13 & $161.3(7)$ \\
\hline F16 & P3 & F14 & $81.8(6)$ \\
\hline F16 & P3 & F18 & $86.7(5)$ \\
\hline F17 & P3 & F13 & $93.1(5)$ \\
\hline F17 & P3 & F14 & $177.1(6)$ \\
\hline F17 & P3 & F15 & $100.0(5)$ \\
\hline F17 & P3 & F16 & $100.3(5)$ \\
\hline
\end{tabular}


Table S11: Hydrogen Fractional Atomic Coordinates $\left(\times 10^{4}\right)$ and Equivalent Isotropic Displacement Parameters $\left(\AA^{2} \times 10^{3}\right)$ for PL15015_sq. $U_{e q}$ is defined as $1 / 3$ of the trace of the orthogonalised $U_{i j}$.

\begin{tabular}{|c|c|c|c|c|}
\hline Atom & & & & $U_{e q}$ \\
\hline$\overline{\mathrm{H} 1}$ & 12031 & 7145 & 6933 & 75 \\
\hline $\mathrm{H} 2$ & 12547 & 7064 & 6830 & 77 \\
\hline H3 & 11821 & 6718 & 6730 & 71 \\
\hline $\mathrm{H} 4$ & 10552 & 6418 & 6738 & 69 \\
\hline H7 & 9399 & 6219 & 6756 & 101 \\
\hline H8 & 8183 & 5927 & 6786 & 102 \\
\hline H10 & 8829 & 6287 & 6983 & 42 \\
\hline H13 & 6539 & 6533 & 6945 & 81 \\
\hline H16 & 7297 & 4791 & 6933 & 106 \\
\hline H17A & 8260 & 7463 & 6932 & 270 \\
\hline H17B & 7632 & 7432 & 6881 & 270 \\
\hline $\mathrm{H} 17 \mathrm{C}$ & 8221 & 7200 & 6854 & 270 \\
\hline H18A & 6185 & 3849 & 6971 & 328 \\
\hline H18B & 5450 & 3924 & 6966 & 328 \\
\hline $\mathrm{H} 18 \mathrm{C}$ & 5919 & 4141 & 7036 & 328 \\
\hline H19 & 4817 & 4323 & 6944 & 42 \\
\hline $\mathrm{H} 21$ & 5874 & 6254 & 7052 & 59 \\
\hline $\mathrm{H} 22$ & 4714 & 6004 & 7095 & 57 \\
\hline $\mathrm{H} 25$ & 3589 & 5537 & 7148 & 55 \\
\hline H26 & 2348 & 5052 & 7183 & 64 \\
\hline $\mathrm{H} 27$ & 1392 & 4005 & 7128 & 60 \\
\hline H 28 & 1689 & 3456 & 7037 & 50 \\
\hline H29 & 11189 & 8114 & 6875 & 61 \\
\hline H30 & 11160 & 9204 & 6857 & 78 \\
\hline H31 & 10526 & 9557 & 6935 & 87 \\
\hline H32 & 9950 & 8790 & 7032 & 74 \\
\hline H35 & 9651 & 8128 & 7129 & 61 \\
\hline H36 & 9223 & 7261 & 7219 & 61 \\
\hline H38 & 9713 & 5920 & 7107 & 43 \\
\hline H40 & 8390 & 5051 & 7178 & 63 \\
\hline H43 & 9617 & 5848 & 7389 & 66 \\
\hline $\mathrm{H} 45 \mathrm{~A}$ & 10109 & 7339 & 7302 & 137 \\
\hline $\mathrm{H} 45 \mathrm{~B}$ & 10636 & 7043 & 7271 & 137 \\
\hline $\mathrm{H} 45 \mathrm{C}$ & 10444 & 6963 & 7351 & 137 \\
\hline $\mathrm{H} 41 \mathrm{~A}$ & 8035 & 4220 & 7270 & 69 \\
\hline $\mathrm{H} 44 \mathrm{~A}$ & 9956 & 6693 & 7298 & 62 \\
\hline $\mathrm{H} 45 \mathrm{D}$ & 9535 & 5977 & 7451 & 154 \\
\hline $\mathrm{H} 45 \mathrm{E}$ & 10239 & 6529 & 7405 & 154 \\
\hline $\mathrm{H} 45 \mathrm{~F}$ & 10091 & 5707 & 7423 & 154 \\
\hline $\mathrm{H} 46 \mathrm{~A}$ & 7720 & 3835 & 7209 & 134 \\
\hline $\mathrm{H} 46 \mathrm{~B}$ & 7278 & 3868 & 7275 & 134 \\
\hline $\mathrm{H} 46 \mathrm{C}$ & 7828 & 3533 & 7281 & 134 \\
\hline H46D & 7794 & 4357 & 7166 & 299 \\
\hline $\mathrm{H} 46 \mathrm{E}$ & 7879 & 5156 & 7149 & 299 \\
\hline $\mathrm{H} 46 \mathrm{~F}$ & 8471 & 4941 & 7119 & 299 \\
\hline H47 & 7492 & 4289 & 7406 & 46 \\
\hline H49 & 9516 & 4428 & 7411 & 61 \\
\hline H50 & 9097 & 3580 & 7504 & 57 \\
\hline H53 & 8538 & 2656 & 7580 & 71 \\
\hline H54 & 7904 & 1814 & 7670 & 100 \\
\hline H55 & 6732 & 1603 & 7704 & 71 \\
\hline H56 & 6197 & 2216 & 7648 & 49 \\
\hline
\end{tabular}




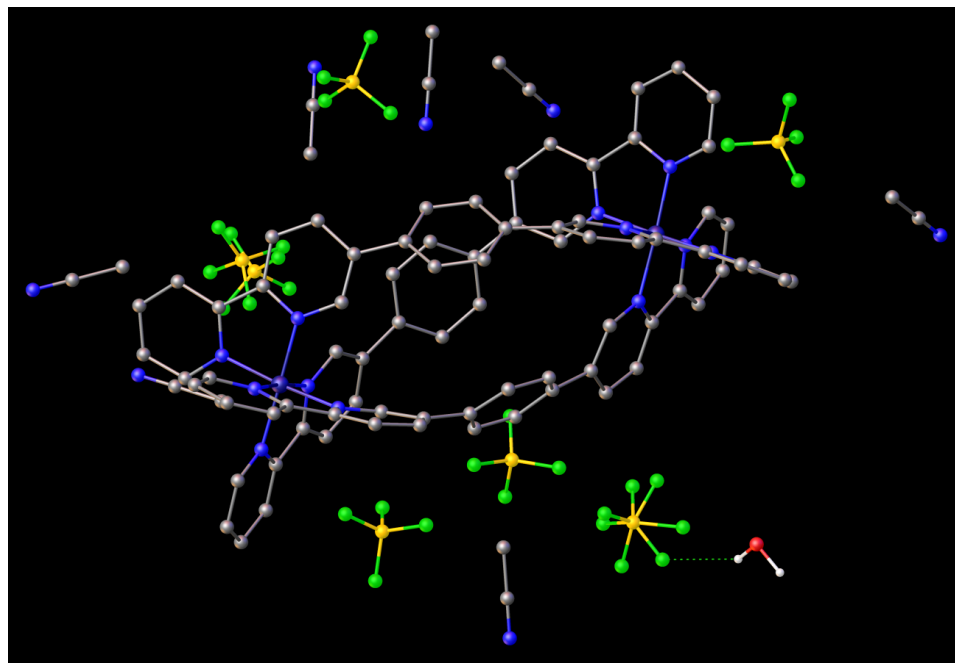

Experimental. Single yellow block-shaped crystals of (PL15013) were recrystallised from a mixture of acetonitrile and diisopropyl ether by vapour diffusion. A suitable crystal $\left(0.27 \times 0.20 \times 0.08 \mathrm{~mm}^{3}\right)$ was selected and mounted on a MITIGEN holder in Paratone oil on a Rigaku Oxford Diffraction SuperNova diffractometer. The crystal was kept at $T=120.0 \mathrm{~K}$ during data collection. Using Olex2, ${ }^{[3]}$ the structure was solved with the ShelXT structure solution program, using the Direct Methods solution method. ${ }^{[4]}$ The model was refined with version of ShelXL using Least Squares minimisation. ${ }^{[5]}$

Crystal Data. $\mathrm{C}_{92} \mathrm{H}_{76} \mathrm{~B}_{6} \mathrm{Co}_{2} \mathrm{~F}_{24} \mathrm{~N}_{19} \mathrm{O}_{0.5}, \quad M_{r}=$ 2094.43, triclinic, P-1 (No. 2), $\mathrm{a}=15.00712(20) \AA, \mathrm{b}=16.8669$ (2) $\AA$, $\mathrm{c}=20.4390(3) \AA, \alpha=73.1727(12)^{\circ}, \beta=68.6423(12)^{\circ}, \gamma=$ 80.3164(11) $)^{\circ}, V=4600.52(11) \AA^{3}, T=120.0 \mathrm{~K}, Z=2, Z^{\prime}=1$, $\mu\left(\mathrm{CuK}_{\alpha}\right)=3.772,151138$ reflections measured, 19157 unique $\left(R_{\text {int }}=0.0763\right)$ which were used in all calculations. The final $w R_{2}$ was 0.1539 (all data) and $R_{l}$ was 0.0552 (I $>2(\mathrm{I})$ ).

\section{Compound}

Formula

$D_{\text {calc. }} / \mathrm{g} \mathrm{cm}^{-3}$

$\mu / \mathrm{mm}^{-1}$

Formula Weight

Colour

Shape

Max Size/mm

Mid Size/mm

Min Size/mm

$T / \mathrm{K}$

Crystal System

Space Group

$a / \AA$

$b / \AA$

$c / \AA ̊$

$\alpha l^{\circ}$

$\beta \rho^{\circ}$

$\mathrm{V} / \AA^{3}$

Z

$Z^{\prime}$

$\theta_{\min } I^{\circ}$

$\theta_{\max } l^{\circ}$

Measured Refl.

Independent Refl.

Reflections Used

$R_{\text {int }}$

Parameters

Restraints

Largest Peak

Deepest Hole

GooF

$w R_{2}$ (all data)

$w R_{2}$

$R_{l}$ (all data)

$R_{l}$

\section{PL15013}

$\mathrm{C}_{92} \mathrm{H}_{76} \mathrm{~B}_{6} \mathrm{Co}_{2} \mathrm{~F}_{24} \mathrm{~N}_{19} \mathrm{O}$

0.5

1.512

3.772

2094.43

yellow

block

0.27

0.20

0.08

120.0

triclinic

P-1

15.00712(20)

$16.8669(2)$

20.4390(3)

$73.1727(12)$

68.6423(12)

$80.3164(11)$

4600.52(11)

2

1

3.163

76.249

151138

19157

17903

0.0763

1382

163

1.346

$-0.758$

1.027

0.1539

0.1498

0.0584

0.0552 


\begin{tabular}{|c|c|c|c|c|}
\hline Reflections: & $\mathrm{d} \min$ & $0.79^{1 / \sigma}$ & $22.4^{\text {Rint }}$ & $7.63 \%$ complete $2.100 \%$ \\
\hline Refinement: & Shift/esd & -0.001 Max Peak & 4 Min Peak & $-0.8^{G}$ \\
\hline
\end{tabular}

A yellow block-shaped crystal with dimensions $0.27 \times 0.20 \times 0.08$ was mounted on a MITIGEN holder in Paratone oil. Data were collected using a Rigaku Oxford Diffraction SuperNova diffractometer equipped with an Oxford Cryosystems Cryostream 700+ low-temperature apparatus operating at $T=120.0 \mathrm{~K}$.

Data were measured using $\omega$ scans scans of $1.0^{\circ}$ per frame for $5.0 \mathrm{~s}$ using $\mathrm{CuK}_{\alpha}$ radiation (sealed X-ray tube, $50 \mathrm{kV}, 0.8 \mathrm{~mA}$ ). The total number of runs and images was based on the strategy calculation from the program CrysAlisPro (Agilent, V1.171.37.35e, 2014). The actually achieved resolution was $\theta=76.249$.

Cell parameters were retrieved using the CrysAlisPro (Agilent, V1.171.37.35e, 2014) software and refined using CrysAlisPro (Agilent, V1.171.37.35e, 2014) on 76965 reflections, 51 of the observed reflections. Data reduction was performed using the CrysAlisPro (Agilent, V1.171.37.35e, 2014) software which corrects for Lorentz polarisation. The final completeness is 100.00 out to 76.249 in $\theta$. The absorption coefficient $(\mu)$ of this material is 3.772 and the minimum and maximum transmissions are 0.491 and 0.790 .

The structure was solved in the space group P-1 (\# 2) by Direct Methods using the ShelXT structure solution program and refined by Least Squares using version of ShelXL. ${ }^{[4-5]}$ All non-hydrogen atoms were refined anisotropically. Hydrogen atom positions were calculated geometrically and refined using the riding model. The crystals lose solvent very quickly when removed from the mother liquor. Crystals plus ML were transferred by pipette onto a shallow well microscope slide and a small block crystal was quickly pushed into Paratone oil, picked up and mounted on the diffractometer. This appears to have prevented significant solvent loss as all acetonitrile molecules were easily identified. One peak was assigned as a half-occupied water molecule. The water $\mathrm{H}$ atoms were based on residual density peaks, and restrained in place by Olex2. Some tetrafluoroborate anions were modelled as disordered over two sites as indicated by elongated ellipsoids and residual electron density peaks.

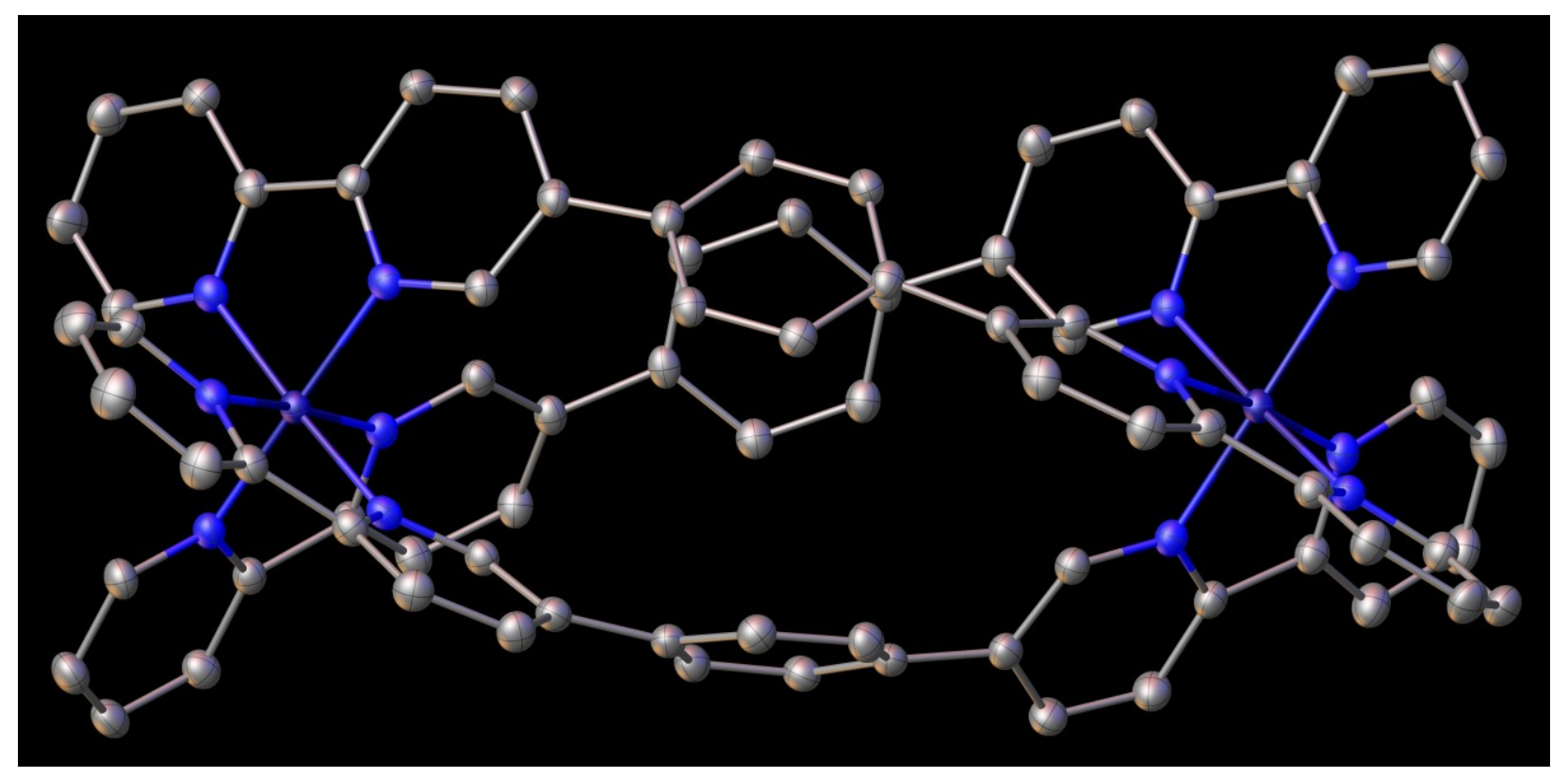

Figure S62: The helicate structure in p115013. Displacement ellipsoids are at the 50pc probability level. 


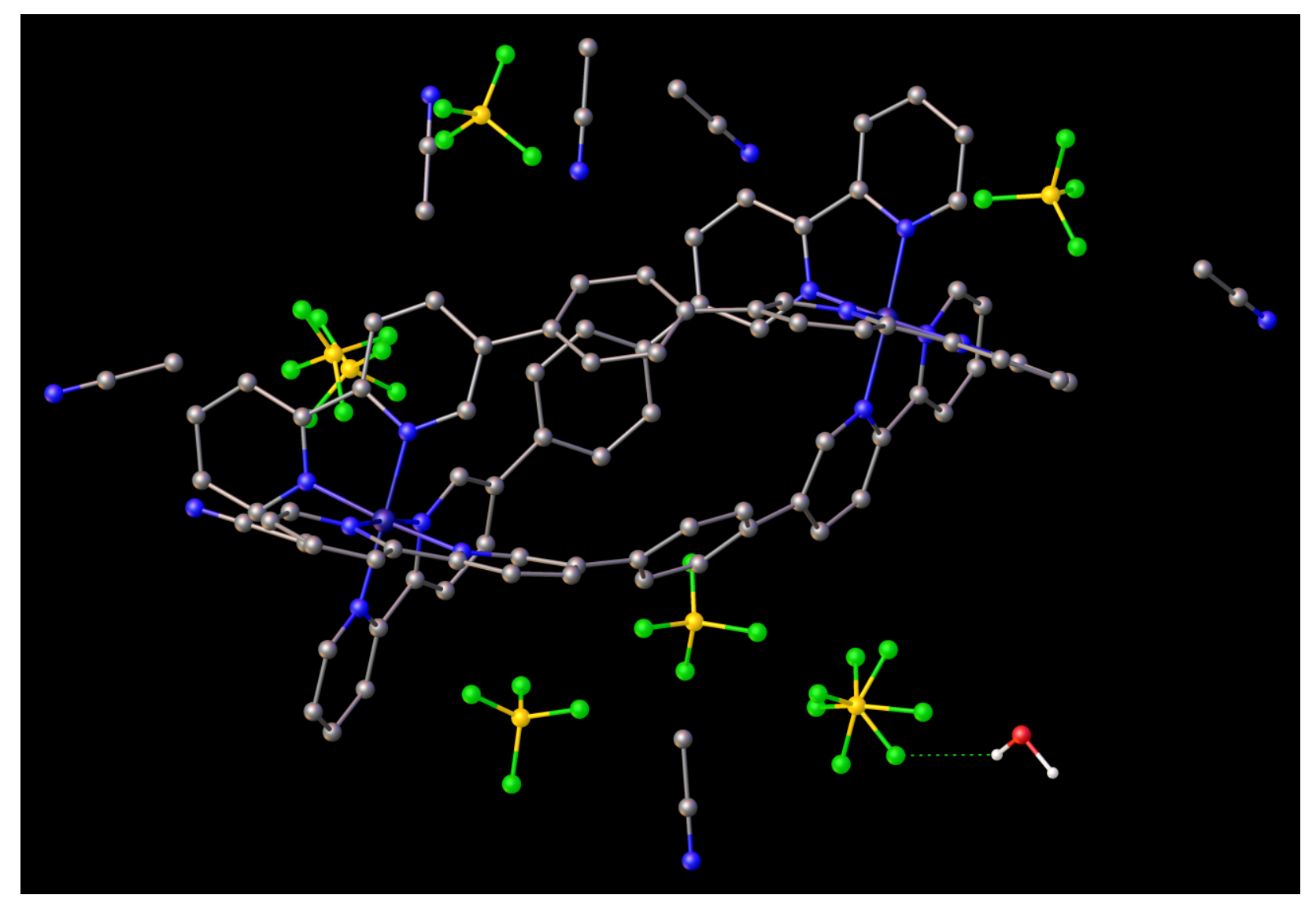

Figure S63: The asymmetric unit of pl15013.

\section{Reflection Statistics}

Total reflections (after filtering) 151138

Completeness

hklsub $>\max </$ sub $>$ collected $\quad(18,21,25)$

$\mathrm{hkl}_{\max }$ used

Lim $d_{\max }$ collected

$\mathrm{d}_{\max }$ used

Friedel pairs

Inconsistent equivalents

$\mathrm{R}_{\text {sigma }}$

Omitted reflections

Multiplicity

Removed systematic absences

0.995

$(18,21,25)$

100.0

13.97

15298

14

0.0297

0

1)

0
(3467, 5522, 6120, 5481, 3936,Maximum multiplicity 20 $3297,2482,1605,1009,604$, $336,214,181,127,46,16,11$,
Unique reflections

Mean $\mathrm{I} / \sigma$

hklsub $>\min </$ sub $>$ collected

$\mathrm{hkl}_{\text {min }}$ used

Lim $d_{\min }$ collected

$\mathrm{d}_{\text {min }}$ used

Friedel pairs merged

$\mathrm{R}_{\text {int }}$

Intensity transformed
Filtered off (Shel/OMIT) 0
19157

22.4

$(-18,-21,-25)$

$(-17,-20,0)$

0.77

0.79

1

0.0763

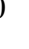

Table S12: Fractional Atomic Coordinates $\left(\times 10^{4}\right)$ and Equivalent Isotropic Displacement Parameters $\left(\AA^{2} \times 10^{3}\right)$ for PL15013. $U_{e q}$ is defined as $1 / 3$ of the trace of the orthogonalised $U_{i j}$.

\begin{tabular}{lclcl}
\hline Atom & $\mathbf{x}$ & $\mathbf{y}$ & $\mathbf{z}$ & $\boldsymbol{U}_{\boldsymbol{e q}}$ \\
\hline Co1 & $1829.2(2)$ & $2193.1(2)$ & $4617.0(2)$ & $16.67(10)$ \\
Co2 & $6982.1(2)$ & $7349.2(2)$ & $1076.3(2)$ & $16.54(10)$ \\
N1 & $708.8(13)$ & $2331.4(11)$ & $5438.2(10)$ & $19.8(4)$ \\
N2 & $2424.9(13)$ & $2773.8(11)$ & $5020.6(10)$ & $18.4(3)$ \\
N3 & $8238.4(14)$ & $7105.6(11)$ & $431.3(10)$ & $20.3(4)$ \\
N4 & $7314.3(13)$ & $6361.7(11)$ & $1734.7(10)$ & $18.6(3)$ \\
N5 & $1136.7(13)$ & $1709.7(12)$ & $4212.7(10)$ & $21.0(4)$ \\
N6 & $1432.1(13)$ & $3221.6(12)$ & $4035.8(10)$ & $19.4(4)$ \\
N7 & $7422.2(13)$ & $8012.8(11)$ & $1507.7(10)$ & $19.8(4)$
\end{tabular}




\begin{tabular}{|c|c|c|c|c|}
\hline Atom & $\mathbf{x}$ & $\mathbf{y}$ & $\mathbf{z}$ & $U_{e q}$ \\
\hline N8 & $5750.8(13)$ & $7543.9(11)$ & 1784(1) & $18.9(4)$ \\
\hline N9 & $2259.6(13)$ & $1139.0(12)$ & $5144.1(10)$ & $20.0(4)$ \\
\hline N10 & $3020.5(13)$ & 2026.3(11) & $3854(1)$ & $18.8(4)$ \\
\hline N11 & $6649.7(13)$ & $8285.5(11)$ & $380.9(10)$ & $19.3(4)$ \\
\hline N12 & 6439.3(13) & $6731.2(11)$ & $670.7(10)$ & $18.3(3)$ \\
\hline $\mathrm{C} 1$ & $-168.5(16)$ & $2066.0(14)$ & $5614.8(13)$ & $22.8(4)$ \\
\hline $\mathrm{C} 2$ & $-906.6(17)$ & 2159.7(15) & $6242.1(13)$ & $26.6(5)$ \\
\hline $\mathrm{C} 3$ & $-752.0(17)$ & $2544.4(16)$ & $6699.1(14)$ & $28.5(5)$ \\
\hline $\mathrm{C} 4$ & $147.0(17)$ & $2834.5(16)$ & $6516.2(13)$ & $26.1(5)$ \\
\hline $\mathrm{C} 5$ & $860.5(16)$ & $2721.7(14)$ & $5882.0(12)$ & $21.2(4)$ \\
\hline C6 & $1824.0(16)$ & $3012.7(14)$ & $5623.2(12)$ & $20.8(4)$ \\
\hline $\mathrm{C} 7$ & $2100.8(17)$ & $3537.8(15)$ & $5912.6(12)$ & $24.5(5)$ \\
\hline $\mathrm{C} 8$ & 2992.7(17) & $3860.2(15)$ & $5560.5(12)$ & $24.3(5)$ \\
\hline C9 & $3618.4(16)$ & $3621.4(14)$ & $4936.6(12)$ & $20.2(4)$ \\
\hline $\mathrm{C} 10$ & $3307.2(15)$ & $3054.2(13)$ & $4706.4(11)$ & $19.0(4)$ \\
\hline $\mathrm{C} 11$ & $4536.7(15)$ & 3999.1(14) & $4475.1(11)$ & $19.9(4)$ \\
\hline $\mathrm{C} 12$ & $5307.9(16)$ & $3537.0(14)$ & $4085.0(13)$ & $23.0(4)$ \\
\hline C13 & $6108.4(16)$ & $3922.4(14)$ & $3565.9(13)$ & $22.9(4)$ \\
\hline $\mathrm{C} 14$ & $6156.2(15)$ & $4782.2(14)$ & $3422.4(12)$ & $20.2(4)$ \\
\hline $\mathrm{C} 15$ & $5409.3(16)$ & $5231.9(14)$ & $3843.4(12)$ & $21.7(4)$ \\
\hline $\mathrm{C} 16$ & $4606.7(16)$ & $4846.4(14)$ & $4364.7(12)$ & $21.8(4)$ \\
\hline $\mathrm{C} 17$ & $8650.1(17)$ & $7537.3(15)$ & $-255.3(13)$ & $25.8(5)$ \\
\hline C18 & $9554.2(18)$ & $7294.5(17)$ & $-683.8(14)$ & $29.9(5)$ \\
\hline C19 & $10049.5(17)$ & $6593.7(16)$ & $-399.9(13)$ & $27.0(5)$ \\
\hline $\mathrm{C} 20$ & $9624.4(16)$ & $6143.2(15)$ & $305.9(13)$ & $23.7(4)$ \\
\hline $\mathrm{C} 21$ & $8717.8(16)$ & $6408.7(13)$ & $708.5(12)$ & $20.3(4)$ \\
\hline $\mathrm{C} 22$ & $8175.7(15)$ & 5973.1(13) & $1448.3(12)$ & $19.4(4)$ \\
\hline $\mathrm{C} 23$ & $8455.3(16)$ & $5199.5(14)$ & $1821.9(13)$ & $24.3(5)$ \\
\hline $\mathrm{C} 24$ & $7832.7(17)$ & $4806.7(14)$ & $2486.6(13)$ & $25.1(5)$ \\
\hline $\mathrm{C} 25$ & $6930.5(16)$ & $5199.8(14)$ & $2780.5(12)$ & $20.3(4)$ \\
\hline $\mathrm{C} 26$ & $6721.0(15)$ & $5990.7(13)$ & 2391.1(12) & $19.3(4)$ \\
\hline $\mathrm{C} 27$ & $1071.8(17)$ & 893.9(14) & $4306.4(13)$ & $24.0(4)$ \\
\hline $\mathrm{C} 28$ & 494.8(19) & 613.9(16) & $4034.8(14)$ & $29.0(5)$ \\
\hline $\mathrm{C} 29$ & $-47.1(18)$ & $1190.5(16)$ & $3664.6(14)$ & $29.6(5)$ \\
\hline $\mathrm{C} 30$ & $35.8(17)$ & $2031.6(16)$ & $3547.6(13)$ & $26.9(5)$ \\
\hline C31 & $645.9(16)$ & $2273.6(14)$ & $3816.4(12)$ & $21.6(4)$ \\
\hline $\mathrm{C} 32$ & $856.3(15)$ & $3132.3(14)$ & $3684.5(12)$ & $20.1(4)$ \\
\hline C33 & $581.4(17)$ & $3805.9(15)$ & $3197.7(13)$ & $24.9(5)$ \\
\hline C34 & $942.3(17)$ & $4568.7(15)$ & $3035.2(13)$ & $24.2(4)$ \\
\hline $\mathrm{C} 35$ & $1558.8(15)$ & $4657.7(14)$ & $3380.8(12)$ & $20.8(4)$ \\
\hline $\mathrm{C} 36$ & $1748.9(15)$ & $3973.7(14)$ & $3898.1(12)$ & $20.5(4)$ \\
\hline $\mathrm{C} 37$ & $2095.2(15)$ & $5400.9(13)$ & $3177.4(12)$ & $20.4(4)$ \\
\hline C38 & $2278.0(16)$ & $5668.1(14)$ & $3700.3(12)$ & $23.1(4)$ \\
\hline C39 & $2899.2(16)$ & $6285.8(14)$ & $3494.4(12)$ & $22.9(4)$ \\
\hline $\mathrm{C} 40$ & $3369.2(15)$ & $6640.6(13)$ & $2759.4(12)$ & $20.6(4)$ \\
\hline C41 & $3156.2(16)$ & $6395.6(14)$ & $2238.0(12)$ & $22.1(4)$ \\
\hline $\mathrm{C} 42$ & $2520.2(16)$ & $5787.8(14)$ & $2442.9(12)$ & $21.8(4)$ \\
\hline $\mathrm{C} 43$ & $8330.6(16)$ & $8185.6(14)$ & $1343.1(13)$ & $24.3(5)$ \\
\hline $\mathrm{C} 44$ & $8572.3(18)$ & $8644.4(16)$ & $1712.0(15)$ & $30.0(5)$ \\
\hline $\mathrm{C} 45$ & $7853(2)$ & $8944.0(17)$ & $2256.1(15)$ & $32.7(5)$ \\
\hline C46 & 6911.4(19) & $8775.1(16)$ & $2423.4(14)$ & $28.4(5)$ \\
\hline $\mathrm{C} 47$ & $6716.1(16)$ & $8296.5(14)$ & $2051.6(12)$ & $21.2(4)$ \\
\hline $\mathrm{C} 48$ & $5772.0(16)$ & $8008.6(13)$ & 2219.3(12) & $20.0(4)$ \\
\hline C49 & $4972.2(17)$ & $8119.0(15)$ & $2810.4(13)$ & $24.7(5)$ \\
\hline $\mathrm{C} 50$ & 4164.1(17) & 7701.3(14) & $2986.0(13)$ & $23.9(4)$ \\
\hline C51 & $4142.2(15)$ & $7200.0(13)$ & $2554.6(12)$ & $20.2(4)$ \\
\hline C52 & $4946.9(16)$ & 7168.6(13) & $1941.5(12)$ & $19.6(4)$ \\
\hline C53 & $1814.2(16)$ & $731.4(14)$ & $5834.7(12)$ & $22.5(4)$ \\
\hline C54 & 2193.3(17) & $-15.1(15)$ & $6166.5(13)$ & $25.2(5)$ \\
\hline C55 & $3067.1(18)$ & $-357.9(15)$ & $5781.8(13)$ & $27.0(5)$ \\
\hline C56 & $3535.5(17)$ & 64.3(14) & $5071.3(13)$ & $25.0(5)$ \\
\hline C57 & $3121.5(16)$ & $810.6(13)$ & $4768.6(12)$ & $20.1(4)$ \\
\hline C58 & $3562.4(16)$ & $1326.8(13)$ & $4037.7(12)$ & $20.1(4)$ \\
\hline C59 & $4482.6(17)$ & $1163.8(14)$ & $3573.6(13)$ & $23.5(4)$ \\
\hline
\end{tabular}




\begin{tabular}{|c|c|c|c|c|}
\hline Atom & $\mathbf{x}$ & $\mathbf{y}$ & $\mathbf{z}$ & $U_{e q}$ \\
\hline$\overline{\mathrm{C} 60}$ & $4873.2(17)$ & $1752.8(14)$ & $2933.8(13)$ & $23.6(4)$ \\
\hline C61 & $4333.4(16)$ & 2491.3(14) & $2752.4(12)$ & $20.3(4)$ \\
\hline C62 & $3392.4(16)$ & $2583.3(14)$ & $3223.0(12)$ & $19.8(4)$ \\
\hline C63 & $4724.9(16)$ & $3205.5(13)$ & $2143.2(11)$ & $20.2(4)$ \\
\hline C64 & $4124.9(17)$ & $3777.4(14)$ & $1816.6(12)$ & $22.6(4)$ \\
\hline C65 & $4460.1(16)$ & $4515.7(14)$ & $1332.6(12)$ & $21.7(4)$ \\
\hline C66 & 5412.1(16) & $4695.8(13)$ & $1154.5(11)$ & $19.9(4)$ \\
\hline C67 & 6028.1(16) & 4104.4(14) & $1447.3(12)$ & $22.4(4)$ \\
\hline C68 & $5688.8(16)$ & $3363.4(14)$ & 1937.1(12) & $22.8(4)$ \\
\hline C69 & $6772.5(17)$ & $9086.0(14)$ & $292.8(13)$ & $23.2(4)$ \\
\hline $\mathrm{C} 70$ & $6437.0(18)$ & $9717.8(15)$ & $-185.0(14)$ & $26.7(5)$ \\
\hline C71 & $5976.2(17)$ & $9527.6(14)$ & $-590.9(13)$ & $25.1(5)$ \\
\hline C72 & $5846.1(17)$ & $8701.0(14)$ & $-503.1(12)$ & $23.3(4)$ \\
\hline C73 & $6186.0(15)$ & 8094.2(13) & $-10.3(12)$ & $19.4(4)$ \\
\hline C74 & $6047.4(15)$ & $7207.7(13)$ & $167.3(11)$ & $18.8(4)$ \\
\hline C75 & $5507.6(17)$ & $6864.4(14)$ & $-93.4(12)$ & $22.5(4)$ \\
\hline C76 & $5316.6(17)$ & $6035.6(14)$ & $199.7(12)$ & $22.8(4)$ \\
\hline C77 & $5697.2(16)$ & $5544.7(13)$ & $732.2(12)$ & $19.8(4)$ \\
\hline $\mathrm{C} 78$ & $6283.5(15)$ & $5918.7(13)$ & $928.2(12)$ & $19.4(4)$ \\
\hline $\mathrm{F} 1$ & $-720.3(11)$ & $6645.3(10)$ & $2180.3(9)$ & $36.5(3)$ \\
\hline $\mathrm{F} 2$ & $291.5(11)$ & $7229.3(10)$ & $1056.6(9)$ & $36.3(3)$ \\
\hline F3 & $846.7(11)$ & $6747.4(11)$ & 2009.7(9) & $38.5(4)$ \\
\hline $\mathrm{F} 4$ & $451.0(13)$ & $5831.7(10)$ & $1548.9(9)$ & $39.4(4)$ \\
\hline B1 & $219(2)$ & 6613.2(18) & 1699.2(15) & $26.9(5)$ \\
\hline F5 & $5711.4(14)$ & $11531.8(12)$ & $1200.0(12)$ & $50.6(5)$ \\
\hline F6 & $6488.6(18)$ & $10677.7(15)$ & 1933.1(13) & $65.0(6)$ \\
\hline F7 & 6999.1(14) & $10661.3(15)$ & $752.1(12)$ & $57.7(6)$ \\
\hline F8 & $5582.7(14)$ & $10131.4(13)$ & $1490.5(12)$ & $53.4(5)$ \\
\hline B2 & $6190.5(19)$ & $10751.3(18)$ & $1372.3(17)$ & $28.3(6)$ \\
\hline F9 & $8892(3)$ & 4691(3) & $4498(2)$ & $63.7(12)$ \\
\hline F9A & $8545(7)$ & $4732(4)$ & $3792(4)$ & $71(2)$ \\
\hline F10 & $8487(4)$ & $4269(7)$ & $3712(3)$ & $129(4)$ \\
\hline F10A & $8533(4)$ & $3428(4)$ & $4017(4)$ & $72(2)$ \\
\hline F11 & $8776(3)$ & $3336(2)$ & $4678(3)$ & $71.7(14)$ \\
\hline F11A & $9053(4)$ & $3987(5)$ & $4703(3)$ & $69(2)$ \\
\hline F12 & $7436.7(13)$ & $4150.8(13)$ & $4825.9(10)$ & $48.9(4)$ \\
\hline B3 & $8396(2)$ & $4079(2)$ & $4392.6(18)$ & $42.7(7)$ \\
\hline F13 & $8690(3)$ & $2908(2)$ & $2548(3)$ & $138.6(18)$ \\
\hline F14 & $8835.9(17)$ & $1609.6(14)$ & $2399.2(15)$ & $70.2(7)$ \\
\hline F15 & $7292(2)$ & $2137(2)$ & $2943.7(15)$ & $107.0(12)$ \\
\hline F16 & $8080.6(18)$ & $2705(2)$ & $1777.0(13)$ & $82.2(9)$ \\
\hline B4 & $8213.2(15)$ & $2353.6(14)$ & $2401.0(11)$ & $13.4(4)$ \\
\hline F17 & $4764.8(13)$ & $2257.2(9)$ & $5716.4(9)$ & $36.3(3)$ \\
\hline F18 & $4873.0(11)$ & $847.9(9)$ & $6076.7(8)$ & $30.5(3)$ \\
\hline F19 & $4878.4(12)$ & $1496.9(10)$ & $4925.4(8)$ & $32.6(3)$ \\
\hline F20 & $3508.2(11)$ & $1537.7(10)$ & $5892.1(9)$ & $35.7(3)$ \\
\hline B5 & $4497(2)$ & $1541.2(17)$ & $5652.1(15)$ & $25.3(5)$ \\
\hline F23 & $2060(2)$ & $2206.6(15)$ & $1469.0(14)$ & $53.7(7)$ \\
\hline F22 & $2120.4(18)$ & $1499.9(13)$ & $2581.4(12)$ & $35.6(5)$ \\
\hline $\mathrm{F} 24$ & $3410.5(16)$ & $2145.1(16)$ & $1731.1(13)$ & $47.7(7)$ \\
\hline F21 & $2094.7(13)$ & $2908.0(11)$ & $2270.9(10)$ & $29.7(5)$ \\
\hline B6 & $2398(3)$ & $2192(2)$ & 2011(2) & 26.8(8) \\
\hline F21A & $2314(13)$ & $1405(9)$ & $1691(10)$ & $116(6)$ \\
\hline F22A & 2933(8) & 2593(9) & $1399(7)$ & $78(4)$ \\
\hline F23A & $2312(10)$ & $1924(14)$ & $2578(7)$ & $104(6)$ \\
\hline F24A & $1266(6)$ & $2477(7)$ & $1946(6)$ & $57(3)$ \\
\hline B6A & $2183(12)$ & $2160(13)$ & $1905(11)$ & $69(5)$ \\
\hline $\mathrm{O} 1$ & $9651(4)$ & $5517(3)$ & $4792(3)$ & $52.3(11)$ \\
\hline N13 & $-211.3(19)$ & $-597.1(18)$ & $2565.8(15)$ & $44.7(6)$ \\
\hline C79 & $319(2)$ & $-93.2(18)$ & $2298.4(15)$ & $34.5(6)$ \\
\hline $\mathrm{C} 80$ & $987(2)$ & $559.8(19)$ & 1958.5(19) & $43.7(7)$ \\
\hline N14 & $1765.5(16)$ & $5125.6(15)$ & $-251.4(13)$ & $35.1(5)$ \\
\hline C81 & $1829.3(18)$ & $4788.4(16)$ & $304.1(15)$ & $30.6(5)$ \\
\hline C82 & $1917(3)$ & $4348(2)$ & $1003.6(17)$ & $45.7(7)$ \\
\hline
\end{tabular}




\begin{tabular}{lcccc}
\hline Atom & $\mathbf{x}$ & $\mathbf{y}$ & $\mathbf{z}$ & $\boldsymbol{U}_{\boldsymbol{e q}}$ \\
\hline N15 & $3050(2)$ & $6070(2)$ & $580.2(16)$ & $50.3(7)$ \\
C83 & $2753(2)$ & $6584.1(18)$ & $201.5(15)$ & $33.9(6)$ \\
C84 & $2360(2)$ & $7241(2)$ & $-277.0(17)$ & $43.6(7)$ \\
N16 & $3841(2)$ & $8102.0(19)$ & $845.0(17)$ & $60.5(9)$ \\
C85 & $3034(2)$ & $8258.6(17)$ & $980.4(17)$ & $43.0(7)$ \\
C86 & $2006(2)$ & $8474(2)$ & $1162(2)$ & $51.2(8)$ \\
N17 & $10830(3)$ & $10470(3)$ & $337(2)$ & $69.1(9)$ \\
C87 & $10028(3)$ & $10648(2)$ & $559(2)$ & $54.3(9)$ \\
C88 & $9009(3)$ & $10881(3)$ & $864(4)$ & $101(2)$ \\
N18 & $8122(2)$ & $1103.1(17)$ & $5410.7(16)$ & $47.8(6)$ \\
C89 & $7684(2)$ & $1588.4(18)$ & $5111.9(16)$ & $35.2(6)$ \\
C90 & $7122(2)$ & $2201(2)$ & $4729(2)$ & $48.4(8)$ \\
N19 & $2624(2)$ & $-844.4(19)$ & $2458.9(18)$ & $52.7(7)$ \\
C91 & $3023(2)$ & $-465.7(19)$ & $2633.2(18)$ & $42.2(7)$ \\
C92 & $3536(2)$ & $6(2)$ & $2857(2)$ & $47.8(7)$
\end{tabular}

Table S13: Anisotropic Displacement Parameters $\left(\times 10^{4}\right)$ PL15013. The anisotropic displacement factor exponent takes the form: $-2 \pi^{2}\left[h^{2} a^{* 2} \times U_{11}+\ldots+2 h k a * \times b^{*} \times U_{12}\right]$

\begin{tabular}{|c|c|c|c|c|c|c|}
\hline Atom & $U_{11}$ & $U_{22}$ & $U_{33}$ & $\boldsymbol{U}_{23}$ & $U_{13}$ & $U_{12}$ \\
\hline$\overline{C o 1}$ & $17.39(17)$ & $16.90(17)$ & $14.79(17)$ & $-3.13(13)$ & $-4.23(13)$ & $-2.82(12)$ \\
\hline $\mathrm{Co} 2$ & $17.58(17)$ & $15.02(17)$ & $16.55(17)$ & $-4.02(13)$ & $-4.74(13)$ & $-1.96(12)$ \\
\hline N1 & $19.2(8)$ & $19.5(9)$ & $19.3(9)$ & $-4.0(7)$ & $-5.2(7)$ & $-2.0(7)$ \\
\hline N2 & 19.1(8) & $18.8(8)$ & $15.7(8)$ & $-1.9(7)$ & $-5.8(7)$ & $-1.8(7)$ \\
\hline N3 & $22.1(9)$ & $18.1(8)$ & $20.8(9)$ & $-5.5(7)$ & $-6.2(7)$ & $-2.9(7)$ \\
\hline N4 & $21.0(9)$ & $17.5(8)$ & $19.6(9)$ & $-5.9(7)$ & $-7.7(7)$ & $-2.9(7)$ \\
\hline N5 & 21.0(9) & 22.1(9) & $17.6(9)$ & $-3.4(7)$ & $-3.2(7)$ & $-5.6(7)$ \\
\hline N6 & $18.0(8)$ & $21.2(9)$ & $17.2(8)$ & $-4.2(7)$ & $-3.7(7)$ & $-3.2(7)$ \\
\hline N7 & $21.2(9)$ & $17.9(8)$ & $20.4(9)$ & $-2.8(7)$ & $-8.2(7)$ & $-2.3(7)$ \\
\hline N8 & 22.1(9) & $16.0(8)$ & $18.7(9)$ & $-4.4(7)$ & $-7.5(7)$ & $0.0(7)$ \\
\hline N9 & $21.7(9)$ & $19.5(9)$ & $18.8(9)$ & $-4.2(7)$ & $-6.0(7)$ & $-4.4(7)$ \\
\hline N10 & $20.8(9)$ & $18.9(8)$ & 17.7(9) & $-5.4(7)$ & $-6.3(7)$ & $-3.3(7)$ \\
\hline N11 & $19.3(8)$ & $16.5(8)$ & $20.0(9)$ & $-4.4(7)$ & $-3.7(7)$ & $-2.6(7)$ \\
\hline N12 & $17.8(8)$ & $18.1(8)$ & $16.6(8)$ & $-4.2(7)$ & $-2.6(7)$ & $-2.0(7)$ \\
\hline $\mathrm{C} 1$ & $20.7(10)$ & $24.6(11)$ & $23.0(11)$ & $-5.1(9)$ & $-6.7(9)$ & $-4.3(8)$ \\
\hline $\mathrm{C} 2$ & $20.1(10)$ & $29.3(12)$ & $27.0(12)$ & $-5.8(9)$ & $-4.2(9)$ & $-3.7(9)$ \\
\hline $\mathrm{C} 3$ & $20.9(11)$ & $34.1(13)$ & $25.3(12)$ & $-8.9(10)$ & $-0.1(9)$ & $-3.4(9)$ \\
\hline $\mathrm{C} 4$ & $23.7(11)$ & $32.4(12)$ & $21.6(11)$ & $-9.2(9)$ & $-3.5(9)$ & $-5.0(9)$ \\
\hline $\mathrm{C} 5$ & $20.7(10)$ & $22.8(10)$ & $19(1)$ & $-4.0(8)$ & $-6.0(8)$ & $-2.6(8)$ \\
\hline C6 & $20.3(10)$ & 23.1(10) & $16.5(10)$ & $-3.1(8)$ & $-4.1(8)$ & $-2.5(8)$ \\
\hline $\mathrm{C} 7$ & $25.1(11)$ & $29.6(11)$ & $19(1)$ & $-8.8(9)$ & $-4.3(9)$ & $-4.6(9)$ \\
\hline $\mathrm{C} 8$ & $27.7(11)$ & 27.1(11) & $21.0(11)$ & $-7.9(9)$ & $-8.3(9)$ & $-6.1(9)$ \\
\hline C9 & $20.1(10)$ & $21.2(10)$ & $18.2(10)$ & $-1.4(8)$ & $-6.8(8)$ & $-3.5(8)$ \\
\hline C10 & $18.9(10)$ & $19.9(10)$ & $16.4(10)$ & $-2.8(8)$ & $-5.1(8)$ & $-1.5(8)$ \\
\hline C11 & $20.4(10)$ & $23.3(10)$ & $15.5(9)$ & $-2.3(8)$ & $-5.8(8)$ & $-5.3(8)$ \\
\hline $\mathrm{C} 12$ & $23(1)$ & $19.6(10)$ & $25.0(11)$ & $-2.4(8)$ & $-8.1(9)$ & $-3.1(8)$ \\
\hline $\mathrm{C} 13$ & $20.7(10)$ & $21.6(10)$ & $23.9(11)$ & $-3.4(9)$ & $-6.3(9)$ & $-1.6(8)$ \\
\hline C14 & $19.2(10)$ & $22.1(10)$ & $18.9(10)$ & $-2.0(8)$ & $-6.9(8)$ & $-4.6(8)$ \\
\hline C15 & $23.9(10)$ & $21.2(10)$ & $20.7(10)$ & $-4.3(8)$ & $-7.5(9)$ & $-4.9(8)$ \\
\hline C16 & $22.6(10)$ & $24.3(11)$ & $19.3(10)$ & $-6.4(8)$ & $-6.7(8)$ & $-2.4(8)$ \\
\hline $\mathrm{C} 17$ & $24.5(11)$ & $25.7(11)$ & $22.6(11)$ & $-2.6(9)$ & $-4.8(9)$ & $-3.0(9)$ \\
\hline C18 & $26.3(12)$ & $32.8(13)$ & $21.7(11)$ & $-2.5(10)$ & $-1.0(9)$ & $-2.1(10)$ \\
\hline C19 & $23.3(11)$ & $30.5(12)$ & $23.6(11)$ & $-7.6(9)$ & $-2.8(9)$ & $-2.3(9)$ \\
\hline $\mathrm{C} 20$ & $20.9(10)$ & $24.1(11)$ & $24.7(11)$ & $-5.2(9)$ & $-6.5(9)$ & $-1.8(8)$ \\
\hline $\mathrm{C} 21$ & $22.2(10)$ & $18.9(10)$ & $20.3(10)$ & $-4.6(8)$ & $-6.9(8)$ & $-4.0(8)$ \\
\hline $\mathrm{C} 22$ & $18(1)$ & $19.8(10)$ & $20.5(10)$ & $-5.0(8)$ & $-5.8(8)$ & $-3.2(8)$ \\
\hline $\mathrm{C} 23$ & $19.7(10)$ & $21.3(10)$ & $26.9(12)$ & $-2.3(9)$ & $-5.3(9)$ & $-0.5(8)$ \\
\hline $\mathrm{C} 24$ & $23.0(11)$ & $21.2(10)$ & $27.3(12)$ & $-0.4(9)$ & $-8.3(9)$ & $-1.5(8)$ \\
\hline $\mathrm{C} 25$ & $20.6(10)$ & $20.7(10)$ & $20.1(10)$ & $-3.2(8)$ & $-7.2(8)$ & $-4.9(8)$ \\
\hline $\mathrm{C} 26$ & $20.7(10)$ & $19(1)$ & $18.5(10)$ & $-4.2(8)$ & $-6.3(8)$ & $-3.3(8)$ \\
\hline $\mathrm{C} 27$ & $28.5(11)$ & $21(1)$ & $21.4(11)$ & $-4.9(8)$ & $-5.9(9)$ & $-5.1(8)$ \\
\hline $\mathrm{C} 28$ & $34.0(13)$ & $26.7(12)$ & $26.9(12)$ & $-6.2(9)$ & $-7.2(10)$ & $-11.4(10)$ \\
\hline $\mathrm{C} 29$ & $29.7(12)$ & $33.4(13)$ & $28.6(12)$ & $-6.6(10)$ & $-9.5(10)$ & $-12.4(10)$ \\
\hline
\end{tabular}




\begin{tabular}{|c|c|c|c|c|c|c|}
\hline Atom & $U_{11}$ & $U_{22}$ & $U_{33}$ & $\boldsymbol{U}_{23}$ & $U_{13}$ & $U_{12}$ \\
\hline$\overline{\mathrm{C} 30}$ & $25.6(11)$ & $31.2(12)$ & $25.1(11)$ & $-5.1(9)$ & $-8.9(9)$ & $-7.4(9)$ \\
\hline C31 & $21(1)$ & $24.7(11)$ & $18(1)$ & $-3.8(8)$ & $-4.9(8)$ & $-5.1(8)$ \\
\hline $\mathrm{C} 32$ & $17.2(9)$ & $23.2(11)$ & $18.6(10)$ & $-4.2(8)$ & $-4.2(8)$ & $-3.9(8)$ \\
\hline $\mathrm{C} 33$ & $23.8(11)$ & $27.1(11)$ & $23.9(11)$ & $-2.9(9)$ & $-10.1(9)$ & $-3.7(9)$ \\
\hline C34 & $23.9(11)$ & $24.6(11)$ & $23.0(11)$ & $-2.1(9)$ & $-9.4(9)$ & $-2.0(8)$ \\
\hline $\mathrm{C} 35$ & $18.6(10)$ & $21(1)$ & $19.7(10)$ & $-4.2(8)$ & $-3.3(8)$ & $-1.9(8)$ \\
\hline $\mathrm{C} 36$ & $19.3(10)$ & $21.4(10)$ & $19.9(10)$ & $-5.1(8)$ & $-5.1(8)$ & $-2.5(8)$ \\
\hline C37 & $18.4(9)$ & $18.3(10)$ & $22.4(11)$ & $-3.4(8)$ & $-6.3(8)$ & $0.1(8)$ \\
\hline C38 & $24.1(11)$ & $23(1)$ & $18.7(10)$ & $-3.5(8)$ & $-3.9(8)$ & $-2.9(8)$ \\
\hline C39 & $22.9(10)$ & $24.8(11)$ & 21.1(11) & $-7.2(9)$ & $-6.4(9)$ & $-1.3(8)$ \\
\hline $\mathrm{C} 40$ & $18.5(10)$ & $18.8(10)$ & $22.1(11)$ & $-3.8(8)$ & $-5.3(8)$ & $-0.3(8)$ \\
\hline $\mathrm{C} 41$ & $22.6(10)$ & $21.3(10)$ & $19.3(10)$ & $-2.3(8)$ & $-5.7(8)$ & $-1.8(8)$ \\
\hline $\mathrm{C} 42$ & $22.5(10)$ & $20.3(10)$ & $22.5(11)$ & $-4.4(8)$ & $-8.7(9)$ & $-0.3(8)$ \\
\hline $\mathrm{C} 43$ & $21.5(10)$ & $23.6(11)$ & $28.5(12)$ & $-6.4(9)$ & $-8.6(9)$ & $-2.9(8)$ \\
\hline $\mathrm{C} 44$ & $26.6(12)$ & $31.4(12)$ & $36.8(14)$ & $-11.0(11)$ & $-12.6(10)$ & $-5.9(10)$ \\
\hline $\mathrm{C} 45$ & $35.4(13)$ & $36.6(13)$ & $33.3(13)$ & $-14.2(11)$ & $-12.6(11)$ & $-9.2(11)$ \\
\hline $\mathrm{C} 46$ & $31.7(12)$ & $30.2(12)$ & $27.6(12)$ & $-12.5(10)$ & $-9(1)$ & $-6.1(10)$ \\
\hline $\mathrm{C} 47$ & $24.4(11)$ & $19.3(10)$ & $19.2(10)$ & $-3.5(8)$ & $-6.9(8)$ & $-3.3(8)$ \\
\hline $\mathrm{C} 48$ & $23.3(10)$ & $18.1(10)$ & $19.2(10)$ & $-5.6(8)$ & $-7.2(8)$ & $-1.3(8)$ \\
\hline C49 & $26.7(11)$ & $25.6(11)$ & $23.0(11)$ & $-9.9(9)$ & $-6.9(9)$ & $-1.4(9)$ \\
\hline $\mathrm{C} 50$ & $24.1(11)$ & $24.7(11)$ & $21.2(11)$ & $-8.2(9)$ & $-3.9(9)$ & $-1.6(9)$ \\
\hline C51 & $20.4(10)$ & $18.9(10)$ & $20.3(10)$ & $-4.3(8)$ & $-6.6(8)$ & $-0.4(8)$ \\
\hline C52 & $21.1(10)$ & $17.6(9)$ & $19.8(10)$ & $-4.8(8)$ & $-6.1(8)$ & $-1.9(8)$ \\
\hline C53 & $24.2(10)$ & $23.3(11)$ & 18.1(10) & $-2.7(8)$ & $-5.1(8)$ & $-5.3(8)$ \\
\hline C54 & $29.1(12)$ & $23.9(11)$ & $19.7(10)$ & $-0.3(9)$ & $-6.7(9)$ & $-6.4(9)$ \\
\hline C55 & $32.0(12)$ & $21.8(11)$ & $24.6(12)$ & $-1.6(9)$ & $-9.8(10)$ & $-2.1(9)$ \\
\hline C56 & $27.3(11)$ & $20.9(10)$ & $23.7(11)$ & $-3.9(9)$ & $-6.6(9)$ & $-1.4(9)$ \\
\hline C57 & $22.6(10)$ & $18.8(10)$ & $18.6(10)$ & $-4.7(8)$ & $-5.6(8)$ & $-3.7(8)$ \\
\hline C58 & $23.3(10)$ & $17.6(10)$ & $19.6(10)$ & $-5.0(8)$ & $-6.2(8)$ & $-3.5(8)$ \\
\hline C59 & $24.5(11)$ & $19.5(10)$ & $22.4(11)$ & $-5.2(8)$ & $-3.5(9)$ & $-0.9(8)$ \\
\hline $\mathrm{C} 60$ & $24.4(11)$ & $21.9(11)$ & $21.2(11)$ & $-6.4(9)$ & $-2.4(9)$ & $-2.6(8)$ \\
\hline C61 & $23.7(10)$ & $20.4(10)$ & $17.4(10)$ & $-6.6(8)$ & $-4.2(8)$ & $-5.6(8)$ \\
\hline C62 & $22(1)$ & $20.2(10)$ & $17.4(10)$ & $-4.9(8)$ & $-5.6(8)$ & $-3.7(8)$ \\
\hline C63 & $24.5(10)$ & $19.4(10)$ & $15.3(10)$ & $-5.5(8)$ & $-2.8(8)$ & $-4.6(8)$ \\
\hline C64 & $24.5(10)$ & $24.4(11)$ & $19.5(10)$ & $-5.6(8)$ & $-5.7(8)$ & $-6.8(8)$ \\
\hline C65 & $24.6(11)$ & $22.6(10)$ & 19.1(10) & $-5.3(8)$ & $-7.7(8)$ & $-3.6(8)$ \\
\hline C66 & $25.4(11)$ & $18.5(10)$ & $15.2(9)$ & $-5.9(8)$ & $-3.1(8)$ & $-6.1(8)$ \\
\hline C67 & $22.2(10)$ & $20.8(10)$ & $21.9(10)$ & $-6.2(8)$ & $-3.0(8)$ & $-4.2(8)$ \\
\hline C68 & $22.8(10)$ & $20(1)$ & $21.4(10)$ & $-3.5(8)$ & $-3.2(8)$ & $-2.7(8)$ \\
\hline C69 & $25.9(11)$ & $18.5(10)$ & $24.0(11)$ & $-3.1(8)$ & $-7.2(9)$ & $-4.9(8)$ \\
\hline C70 & $31.3(12)$ & $18.6(10)$ & $27.3(12)$ & $-2.7(9)$ & $-8.1(10)$ & $-2.9(9)$ \\
\hline C71 & $28.0(11)$ & $19.9(10)$ & $22.4(11)$ & $-0.9(8)$ & $-6.5(9)$ & $-0.1(8)$ \\
\hline $\mathrm{C} 72$ & $25.7(11)$ & $22.2(11)$ & $19.5(10)$ & $-4.0(8)$ & $-6.1(9)$ & $-1.1(8)$ \\
\hline C73 & $19.9(10)$ & $18.7(10)$ & $17.9(10)$ & $-4.8(8)$ & $-3.5(8)$ & $-2.5(8)$ \\
\hline C74 & $20(1)$ & $19.4(10)$ & $14.3(9)$ & $-3.7(8)$ & $-2.3(8)$ & $-2.9(8)$ \\
\hline $\mathrm{C} 75$ & $28.2(11)$ & $22(1)$ & $16.6(10)$ & $-3.0(8)$ & $-7.3(8)$ & $-4.1(8)$ \\
\hline C76 & $26.1(11)$ & $25.0(11)$ & $18.5(10)$ & $-5.6(8)$ & $-6.1(8)$ & $-7.5(9)$ \\
\hline C77 & $21.8(10)$ & $18.1(10)$ & $17.2(10)$ & $-5.4(8)$ & $-2.1(8)$ & $-3.8(8)$ \\
\hline C78 & $20.1(10)$ & $18.2(10)$ & $17.5(10)$ & $-4.0(8)$ & $-3.2(8)$ & $-2.3(8)$ \\
\hline $\mathrm{F} 1$ & $29.3(8)$ & $41.3(9)$ & $35.9(8)$ & $-10.9(7)$ & $-5.9(6)$ & $-3.5(6)$ \\
\hline $\mathrm{F} 2$ & $35.7(8)$ & $38.8(8)$ & $30.1(8)$ & $-3.8(6)$ & $-11.3(6)$ & $1.2(6)$ \\
\hline F3 & $33.0(8)$ & $53.6(10)$ & $36.8(8)$ & $-19.7(7)$ & $-16.1(7)$ & $1.0(7)$ \\
\hline F4 & $48.8(9)$ & $34.4(8)$ & 40.1(9) & $-16.7(7)$ & $-19.5(8)$ & $7.7(7)$ \\
\hline B1 & $26.3(13)$ & $31.0(14)$ & $26.1(13)$ & $-10.4(11)$ & $-11.0(11)$ & $1.9(10)$ \\
\hline F5 & $52.5(11)$ & $44.8(10)$ & $64.3(12)$ & $-15.4(9)$ & $-32.7(10)$ & $4.5(8)$ \\
\hline F6 & $81.7(15)$ & $71.0(14)$ & $62.7(13)$ & $-39.2(11)$ & $-46.1(12)$ & $30.9(12)$ \\
\hline F7 & $40.1(10)$ & $80.9(15)$ & $59.3(12)$ & $-44.0(11)$ & $0.6(9)$ & $-15.9(9)$ \\
\hline F8 & $42.4(10)$ & $51.9(11)$ & $59.7(12)$ & $-14.1(9)$ & $-5.6(9)$ & $-13.0(8)$ \\
\hline B2 & $22.0(12)$ & $29.4(13)$ & $37.6(15)$ & $-16.5(12)$ & $-9.7(11)$ & $1.5(10)$ \\
\hline F9 & 47.6(19) & $59(2)$ & $64(2)$ & $-4.6(18)$ & $-2.9(16)$ & $-3.1(16)$ \\
\hline F9A & $68(4)$ & 63(4) & $50(4)$ & $5(3)$ & $-1(3)$ & $6(3)$ \\
\hline F10 & $49(3)$ & $298(11)$ & $33(2)$ & $-43(4)$ & $-15.8(19)$ & $17(6)$ \\
\hline F10A & $43(3)$ & $71(4)$ & $91(5)$ & $-60(4)$ & $29(3)$ & $-27(3)$ \\
\hline F11 & $49(2)$ & $50(2)$ & $120(4)$ & $-21(2)$ & $-39(2)$ & 13.1(15) \\
\hline
\end{tabular}




\begin{tabular}{|c|c|c|c|c|c|c|}
\hline Atom & $U_{11}$ & $U_{22}$ & $U_{33}$ & $\boldsymbol{U}_{23}$ & $U_{13}$ & $U_{12}$ \\
\hline F11A & $49(3)$ & $116(7)$ & $42(3)$ & $-25(3)$ & $-17(2)$ & $7(3)$ \\
\hline F12 & $38.4(9)$ & $61.5(12)$ & $34.8(9)$ & $-12.6(8)$ & $-2.4(7)$ & $7.3(8)$ \\
\hline B3 & $34.1(15)$ & $56.4(19)$ & 31.1(15) & $-13.1(14)$ & $-8.2(12)$ & $11.5(13)$ \\
\hline F13 & $206(4)$ & $75.3(18)$ & $242(5)$ & $-93(2)$ & $-193(4)$ & $71(2)$ \\
\hline F14 & $55.4(12)$ & $44.5(11)$ & $92.5(18)$ & $-13.6(11)$ & $-14.9(12)$ & $15.4(10)$ \\
\hline F15 & $91.9(19)$ & $94(2)$ & $53.8(14)$ & $16.6(14)$ & $30.5(14)$ & $20.6(16)$ \\
\hline F16 & $61.7(14)$ & $124(2)$ & $44.3(12)$ & $24.0(13)$ & $-23.7(11)$ & $-28.6(14)$ \\
\hline B4 & $13.5(9)$ & $19.2(10)$ & 7.3(9) & $-8.1(8)$ & $-5.9(7)$ & $13.0(8)$ \\
\hline F17 & $51.0(9)$ & $25.7(7)$ & $37.7(8)$ & $-6.6(6)$ & $-21.5(7)$ & $-4.1(7)$ \\
\hline F18 & $39.8(8)$ & $25.0(7)$ & $24.2(7)$ & $-5.2(5)$ & $-12.3(6)$ & $6.0(6)$ \\
\hline F19 & 41.1(8) & $33.7(8)$ & $21.3(7)$ & $-4.2(6)$ & $-9.6(6)$ & $-5.1(6)$ \\
\hline F20 & $30.2(8)$ & $43.6(9)$ & $31.9(8)$ & $-7.0(7)$ & $-11.4(6)$ & $-0.9(6)$ \\
\hline B5 & $30.2(13)$ & $24.3(12)$ & $21.0(12)$ & $-4.1(10)$ & $-10(1)$ & $-0.1(10)$ \\
\hline F23 & $83.6(19)$ & $48.7(14)$ & $48.0(14)$ & $-12.3(11)$ & $-42.2(14)$ & $-8.8(12)$ \\
\hline F22 & $38.6(12)$ & $26.4(10)$ & $32.7(11)$ & $0.5(8)$ & $-6.3(9)$ & $-5.1(8)$ \\
\hline F24 & $37.1(12)$ & $55.6(14)$ & $47.1(13)$ & $-27.2(11)$ & $5.6(10)$ & $-14.3(10)$ \\
\hline F21 & $32.2(9)$ & $27.0(9)$ & $29.0(9)$ & $-9.9(7)$ & $-6.2(7)$ & $-4.3(7)$ \\
\hline B6 & $40(2)$ & $24.6(16)$ & $14.9(17)$ & $-4.6(13)$ & $-4.7(14)$ & $-11.9(14)$ \\
\hline $\mathrm{F} 21 \mathrm{~A}$ & $113(11)$ & $106(9)$ & $117(12)$ & $-28(8)$ & $-27(9)$ & $-4(7)$ \\
\hline F22A & $41(5)$ & 102(9) & $66(7)$ & $-9(6)$ & $1(5)$ & $0(5)$ \\
\hline F23A & $53(8)$ & $172(16)$ & $66(8)$ & $-19(8)$ & $-17(6)$ & $20(9)$ \\
\hline F24A & $47(5)$ & $64(6)$ & $48(6)$ & $17(5)$ & $-27(4)$ & $-6(4)$ \\
\hline B6A & $45(7)$ & $89(10)$ & $61(9)$ & $-1(7)$ & $-19(6)$ & $-2(6)$ \\
\hline $\mathrm{O} 1$ & $52(3)$ & $48(3)$ & $49(3)$ & $-8(2)$ & $-16(2)$ & $7(2)$ \\
\hline N13 & $37.5(13)$ & $48.1(15)$ & $42.7(14)$ & $-10.9(12)$ & $-4.6(11)$ & $-8.0(11)$ \\
\hline C79 & $35.7(14)$ & $35.4(14)$ & $31.5(13)$ & $-10.8(11)$ & $-10.5(11)$ & $2.5(12)$ \\
\hline $\mathrm{C} 80$ & $46.6(17)$ & $35.8(15)$ & 47.9(17) & $-6.0(13)$ & $-16.0(14)$ & $-8.1(13)$ \\
\hline N14 & $29.6(11)$ & $39.3(12)$ & $35.2(13)$ & $-12.3(10)$ & $-7.9(9)$ & $-0.8(9)$ \\
\hline C81 & $25.6(11)$ & $27.6(12)$ & $36.6(14)$ & $-12.8(11)$ & $-6(1)$ & $2.0(9)$ \\
\hline $\mathrm{C} 82$ & $53.8(18)$ & $40.0(16)$ & $36.7(16)$ & $-5.0(13)$ & $-14.4(14)$ & $4.7(13)$ \\
\hline N15 & $41.3(14)$ & $59.5(17)$ & $44.7(15)$ & $0.9(13)$ & $-19.2(12)$ & $-2.3(12)$ \\
\hline $\mathrm{C} 83$ & $32.3(13)$ & $38.5(14)$ & $29.6(13)$ & $-7.7(11)$ & $-8.3(11)$ & $-4.9(11)$ \\
\hline C84 & $43.7(16)$ & $41.7(16)$ & $37.0(15)$ & $-1.8(13)$ & $-12.7(13)$ & $3.5(13)$ \\
\hline N16 & $53.2(18)$ & $40.6(15)$ & $50.5(17)$ & $12.0(13)$ & $2.5(14)$ & $8.2(13)$ \\
\hline $\mathrm{C} 85$ & $53.4(19)$ & $23.4(12)$ & $36.2(15)$ & $1.5(11)$ & $-2.2(13)$ & $-5.3(12)$ \\
\hline C86 & $45.1(17)$ & $48.1(18)$ & $56(2)$ & $-11.8(15)$ & $-7.8(15)$ & $-13.8(14)$ \\
\hline N17 & $57(2)$ & $89(3)$ & $65(2)$ & $-18.4(19)$ & $-19.2(17)$ & $-18.4(18)$ \\
\hline C87 & $59(2)$ & $40.8(17)$ & $69(2)$ & 4.1(16) & $-36.8(19)$ & $-17.1(15)$ \\
\hline C88 & $60(3)$ & $49(2)$ & $207(7)$ & $-21(3)$ & $-71(4)$ & $3(2)$ \\
\hline N18 & $54.3(16)$ & $39.7(14)$ & $52.6(16)$ & $-5.6(12)$ & $-25.4(14)$ & $-5.0(12)$ \\
\hline C89 & $34.9(13)$ & $35.6(14)$ & $36.5(14)$ & $-9.1(11)$ & $-11.3(11)$ & $-7.5(11)$ \\
\hline C90 & $46.5(17)$ & $44.3(17)$ & $59(2)$ & $-10.3(15)$ & $-27.7(16)$ & $4.2(14)$ \\
\hline N19 & $57.1(17)$ & $46.3(15)$ & $57.0(18)$ & $-9.6(13)$ & $-24.4(15)$ & $-3.4(13)$ \\
\hline C91 & $40.3(15)$ & $37.3(15)$ & $42.3(16)$ & $-2.8(12)$ & $-12.0(13)$ & $-1.8(12)$ \\
\hline $\mathrm{C} 92$ & $45.7(17)$ & $42.4(17)$ & $56(2)$ & $-11.0(15)$ & $-19.3(15)$ & $-2.3(13)$ \\
\hline
\end{tabular}

Table S14: Bond Lengths in Å for PL15013.

\begin{tabular}{|c|c|c|}
\hline Atom & Atom & Length/Å \\
\hline$\overline{\mathrm{Co} 1}$ & N1 & $1.9334(19)$ \\
\hline Col & N2 & $1.9335(19)$ \\
\hline Col & N5 & $1.9309(19)$ \\
\hline Col & N6 & $1.9365(19)$ \\
\hline Col & N9 & $1.9366(19)$ \\
\hline Col & N10 & $1.9393(19)$ \\
\hline $\mathrm{Co} 2$ & N3 & $1.9245(19)$ \\
\hline $\mathrm{Co} 2$ & N4 & $1.9353(19)$ \\
\hline $\mathrm{Co} 2$ & N7 & $1.9263(19)$ \\
\hline $\mathrm{Co} 2$ & N8 & $1.9336(19)$ \\
\hline $\mathrm{Co} 2$ & N11 & $1.9274(19)$ \\
\hline $\mathrm{Co} 2$ & $\mathrm{~N} 12$ & $1.9276(19)$ \\
\hline N1 & $\mathrm{C} 1$ & $1.350(3)$ \\
\hline N1 & $\mathrm{C} 5$ & $1.362(3)$ \\
\hline
\end{tabular}

\begin{tabular}{|c|c|c|}
\hline Atom & Atom & Length/Å \\
\hline N2 & C6 & $1.360(3)$ \\
\hline N2 & $\mathrm{C} 10$ & $1.343(3)$ \\
\hline N3 & $\mathrm{C} 17$ & $1.345(3)$ \\
\hline N3 & $\mathrm{C} 21$ & $1.359(3)$ \\
\hline N4 & $\mathrm{C} 22$ & $1.352(3)$ \\
\hline N4 & $\mathrm{C} 26$ & $1.347(3)$ \\
\hline N5 & $\mathrm{C} 27$ & $1.348(3)$ \\
\hline N5 & C31 & $1.362(3)$ \\
\hline N6 & $\mathrm{C} 32$ & $1.358(3)$ \\
\hline N6 & $\mathrm{C} 36$ & $1.347(3)$ \\
\hline N7 & $\mathrm{C} 43$ & $1.341(3)$ \\
\hline N7 & $\mathrm{C} 47$ & $1.366(3)$ \\
\hline N8 & $\mathrm{C} 48$ & $1.356(3)$ \\
\hline N8 & C52 & $1.347(3)$ \\
\hline
\end{tabular}




\begin{tabular}{|c|c|c|}
\hline Atom & Atom & Length/Å \\
\hline N9 & C53 & $1.348(3)$ \\
\hline N9 & C57 & $1.360(3)$ \\
\hline N10 & C58 & $1.356(3)$ \\
\hline N10 & C62 & $1.345(3)$ \\
\hline N11 & C69 & $1.345(3)$ \\
\hline N11 & C73 & $1.363(3)$ \\
\hline N12 & C74 & $1.361(3)$ \\
\hline N12 & C78 & $1.345(3)$ \\
\hline $\mathrm{C} 1$ & $\mathrm{C} 2$ & $1.386(3)$ \\
\hline $\mathrm{C} 2$ & $\mathrm{C} 3$ & $1.381(4)$ \\
\hline $\mathrm{C} 3$ & $\mathrm{C} 4$ & $1.396(3)$ \\
\hline $\mathrm{C} 4$ & $\mathrm{C} 5$ & $1.388(3)$ \\
\hline $\mathrm{C} 5$ & C6 & $1.464(3)$ \\
\hline C6 & $\mathrm{C} 7$ & $1.383(3)$ \\
\hline $\mathrm{C} 7$ & $\mathrm{C} 8$ & $1.386(3)$ \\
\hline $\mathrm{C} 8$ & C9 & $1.403(3)$ \\
\hline C9 & $\mathrm{C} 10$ & $1.388(3)$ \\
\hline C9 & $\mathrm{C} 11$ & $1.482(3)$ \\
\hline $\mathrm{C} 11$ & $\mathrm{C} 12$ & $1.403(3)$ \\
\hline C11 & C16 & $1.397(3)$ \\
\hline $\mathrm{C} 12$ & $\mathrm{C} 13$ & $1.388(3)$ \\
\hline C13 & C14 & $1.403(3)$ \\
\hline $\mathrm{C} 14$ & $\mathrm{C} 15$ & $1.401(3)$ \\
\hline $\mathrm{C} 14$ & $\mathrm{C} 25$ & $1.482(3)$ \\
\hline $\mathrm{C} 15$ & $\mathrm{C} 16$ & $1.392(3)$ \\
\hline $\mathrm{C} 17$ & $\mathrm{C} 18$ & $1.390(3)$ \\
\hline C18 & C19 & $1.381(4)$ \\
\hline C19 & $\mathrm{C} 20$ & $1.387(3)$ \\
\hline $\mathrm{C} 20$ & $\mathrm{C} 21$ & $1.386(3)$ \\
\hline $\mathrm{C} 21$ & $\mathrm{C} 22$ & $1.466(3)$ \\
\hline $\mathrm{C} 22$ & $\mathrm{C} 23$ & $1.391(3)$ \\
\hline $\mathrm{C} 23$ & $\mathrm{C} 24$ & $1.386(3)$ \\
\hline $\mathrm{C} 24$ & $\mathrm{C} 25$ & $1.404(3)$ \\
\hline $\mathrm{C} 25$ & $\mathrm{C} 26$ & $1.392(3)$ \\
\hline $\mathrm{C} 27$ & $\mathrm{C} 28$ & $1.386(3)$ \\
\hline $\mathrm{C} 28$ & $\mathrm{C} 29$ & $1.391(4)$ \\
\hline $\mathrm{C} 29$ & $\mathrm{C} 30$ & $1.388(4)$ \\
\hline $\mathrm{C} 30$ & C31 & $1.389(3)$ \\
\hline C31 & $\mathrm{C} 32$ & $1.462(3)$ \\
\hline C32 & $\mathrm{C} 33$ & $1.392(3)$ \\
\hline C33 & C34 & $1.384(3)$ \\
\hline C34 & $\mathrm{C} 35$ & $1.401(3)$ \\
\hline $\mathrm{C} 35$ & $\mathrm{C} 36$ & $1.390(3)$ \\
\hline C35 & $\mathrm{C} 37$ & $1.478(3)$ \\
\hline C37 & C38 & $1.398(3)$ \\
\hline C37 & $\mathrm{C} 42$ & $1.401(3)$ \\
\hline C38 & C39 & $1.385(3)$ \\
\hline C39 & $\mathrm{C} 40$ & $1.401(3)$ \\
\hline $\mathrm{C} 40$ & $\mathrm{C} 41$ & $1.399(3)$ \\
\hline $\mathrm{C} 40$ & C51 & $1.482(3)$ \\
\hline $\mathrm{C} 41$ & $\mathrm{C} 42$ & $1.388(3)$ \\
\hline $\mathrm{C} 43$ & $\mathrm{C} 44$ & $1.390(3)$ \\
\hline $\mathrm{C} 44$ & $\mathrm{C} 45$ & $1.388(4)$ \\
\hline $\mathrm{C} 45$ & $\mathrm{C} 46$ & $1.386(4)$ \\
\hline $\mathrm{C} 46$ & $\mathrm{C} 47$ & $1.383(3)$ \\
\hline $\mathrm{C} 47$ & $\mathrm{C} 48$ & $1.463(3)$ \\
\hline $\mathrm{C} 48$ & C49 & $1.391(3)$ \\
\hline C49 & $\mathrm{C} 50$ & $1.386(3)$ \\
\hline $\mathrm{C} 50$ & C51 & $1.398(3)$ \\
\hline C51 & C52 & $1.395(3)$ \\
\hline C53 & C54 & $1.384(3)$ \\
\hline C54 & C55 & $1.389(3)$ \\
\hline C55 & C56 & $1.391(3)$ \\
\hline C56 & $\mathrm{C} 57$ & $1.385(3)$ \\
\hline
\end{tabular}

\begin{tabular}{|c|c|c|}
\hline Atom & Atom & Length $/ \AA ̊$ \\
\hline$\overline{\mathrm{C} 57}$ & C58 & $1.466(3)$ \\
\hline C58 & C59 & $1.397(3)$ \\
\hline C59 & C60 & $1.388(3)$ \\
\hline C60 & C61 & $1.399(3)$ \\
\hline C61 & C62 & $1.402(3)$ \\
\hline C61 & C63 & $1.478(3)$ \\
\hline C63 & C64 & $1.400(3)$ \\
\hline C63 & C68 & $1.401(3)$ \\
\hline C64 & C65 & $1.385(3)$ \\
\hline C65 & C66 & $1.403(3)$ \\
\hline C66 & C67 & $1.398(3)$ \\
\hline C66 & C77 & $1.483(3)$ \\
\hline C67 & C68 & $1.394(3)$ \\
\hline C69 & $\mathrm{C} 70$ & $1.385(3)$ \\
\hline $\mathrm{C} 70$ & C71 & $1.384(4)$ \\
\hline C71 & C72 & $1.391(3)$ \\
\hline $\mathrm{C} 72$ & C73 & $1.387(3)$ \\
\hline $\mathrm{C} 73$ & C74 & $1.465(3)$ \\
\hline C74 & C75 & $1.388(3)$ \\
\hline $\mathrm{C} 75$ & C76 & $1.386(3)$ \\
\hline C76 & C77 & $1.403(3)$ \\
\hline $\mathrm{C} 77$ & C78 & $1.387(3)$ \\
\hline $\mathrm{F} 1$ & B1 & $1.397(3)$ \\
\hline $\mathrm{F} 2$ & B1 & $1.400(3)$ \\
\hline F3 & B1 & $1.390(3)$ \\
\hline F4 & B1 & $1.398(3)$ \\
\hline F5 & B2 & $1.404(3)$ \\
\hline F6 & B2 & $1.342(4)$ \\
\hline F7 & B2 & $1.425(3)$ \\
\hline F8 & B2 & $1.413(3)$ \\
\hline F9 & B3 & $1.475(5)$ \\
\hline F9A & B3 & $1.368(7)$ \\
\hline F10 & B3 & $1.294(6)$ \\
\hline F10A & B3 & $1.461(6)$ \\
\hline F11 & B3 & $1.347(5)$ \\
\hline F11A & B3 & $1.323(6)$ \\
\hline F12 & B3 & $1.395(4)$ \\
\hline F13 & B4 & $1.421(4)$ \\
\hline F14 & B4 & $1.433(3)$ \\
\hline F15 & B4 & $1.447(3)$ \\
\hline F16 & B4 & $1.314(3)$ \\
\hline F17 & B5 & $1.389(3)$ \\
\hline F18 & B5 & $1.409(3)$ \\
\hline F19 & B5 & $1.403(3)$ \\
\hline F20 & B5 & $1.384(3)$ \\
\hline F23 & B6 & $1.370(4)$ \\
\hline F22 & B6 & $1.384(4)$ \\
\hline F24 & B6 & $1.412(5)$ \\
\hline F21 & B6 & $1.399(4)$ \\
\hline F21A & B6A & $1.424(19)$ \\
\hline F22A & B6A & $1.364(18)$ \\
\hline F23A & B6A & $1.394(18)$ \\
\hline F24A & B6A & $1.371(16)$ \\
\hline N13 & C79 & $1.138(4)$ \\
\hline C79 & $\mathrm{C} 80$ & $1.457(4)$ \\
\hline N14 & C81 & $1.144(4)$ \\
\hline C81 & $\mathrm{C} 82$ & $1.450(4)$ \\
\hline N15 & C83 & $1.132(4)$ \\
\hline C83 & C84 & $1.451(4)$ \\
\hline N16 & C85 & $1.141(5)$ \\
\hline $\mathrm{C} 85$ & C86 & $1.457(5)$ \\
\hline N17 & C87 & $1.142(5)$ \\
\hline C87 & C88 & $1.459(6)$ \\
\hline N18 & C89 & $1.139(4)$ \\
\hline
\end{tabular}




\begin{tabular}{lll}
\hline Atom & Atom & Length/ $\AA$ \\
\hline C89 & C90 & $1.453(4)$ \\
N19 & C91 & $1.145(5)$ \\
C91 & C92 & $1.451(5)$
\end{tabular}

Table S15: Bond Angles in ${ }^{\circ}$ for PL15013.

\begin{tabular}{|c|c|c|c|c|c|c|c|}
\hline Atom & Atom & Atom & Angle $/^{\circ}$ & Atom & Atom & Atom & Angle $/^{\circ}$ \\
\hline N1 & Co1 & N2 & $83.23(8)$ & $\overline{\mathrm{C} 53}$ & N9 & $\mathrm{C} 57$ & $118.54(19)$ \\
\hline N1 & Col & N6 & $89.62(8)$ & C57 & N9 & Col & $114.58(15)$ \\
\hline N1 & Col & N9 & $93.37(8)$ & C58 & N10 & Col & $114.54(15)$ \\
\hline N1 & Co1 & N10 & $174.71(8)$ & C62 & N10 & Col & $125.79(15)$ \\
\hline $\mathrm{N} 2$ & Co1 & N6 & $92.13(8)$ & C62 & N10 & C58 & $119.01(19)$ \\
\hline $\mathrm{N} 2$ & Col & N9 & $90.16(8)$ & C69 & N11 & $\mathrm{Co} 2$ & $126.23(16)$ \\
\hline N2 & Col & N10 & $92.93(8)$ & C69 & N11 & C73 & 119.11(19) \\
\hline N5 & Co1 & N1 & $93.39(8)$ & C73 & N11 & $\mathrm{Co} 2$ & $114.49(14)$ \\
\hline N5 & Co1 & $\mathrm{N} 2$ & $174.07(8)$ & C74 & N12 & $\mathrm{Co} 2$ & $114.48(14)$ \\
\hline N5 & Col & N6 & $82.95(8)$ & C78 & N12 & $\mathrm{Co} 2$ & $125.22(15)$ \\
\hline N5 & Col & N9 & $94.92(8)$ & C78 & N12 & C74 & $119.29(19)$ \\
\hline N5 & Col & N10 & $90.74(8)$ & N1 & $\mathrm{C} 1$ & $\mathrm{C} 2$ & $121.9(2)$ \\
\hline N6 & Co1 & N9 & $176.42(8)$ & $\mathrm{C} 3$ & $\mathrm{C} 2$ & $\mathrm{C} 1$ & $119.5(2)$ \\
\hline N6 & Col & N10 & $94.16(8)$ & $\mathrm{C} 2$ & $\mathrm{C} 3$ & $\mathrm{C} 4$ & $119.1(2)$ \\
\hline N9 & Col & N10 & $82.98(8)$ & $\mathrm{C} 5$ & $\mathrm{C} 4$ & $\mathrm{C} 3$ & $118.9(2)$ \\
\hline N3 & $\mathrm{Co} 2$ & N4 & $83.10(8)$ & N1 & $\mathrm{C} 5$ & $\mathrm{C} 4$ & $121.8(2)$ \\
\hline N3 & $\mathrm{Co} 2$ & N7 & $94.82(8)$ & N1 & $\mathrm{C} 5$ & C6 & $114.08(19)$ \\
\hline N3 & $\mathrm{Co} 2$ & N8 & $175.70(8)$ & $\mathrm{C} 4$ & $\mathrm{C} 5$ & C6 & $124.1(2)$ \\
\hline N3 & $\mathrm{Co} 2$ & N11 & $95.42(8)$ & $\mathrm{N} 2$ & C6 & $\mathrm{C} 5$ & $113.65(19)$ \\
\hline N3 & $\mathrm{Co} 2$ & $\mathrm{~N} 12$ & $89.54(8)$ & $\mathrm{N} 2$ & C6 & $\mathrm{C} 7$ & $121.5(2)$ \\
\hline N7 & $\mathrm{Co} 2$ & N4 & $89.16(8)$ & $\mathrm{C} 7$ & C6 & $\mathrm{C} 5$ & $124.7(2)$ \\
\hline N7 & $\mathrm{Co} 2$ & N8 & $83.55(8)$ & C6 & $\mathrm{C} 7$ & $\mathrm{C} 8$ & $119.3(2)$ \\
\hline N7 & $\mathrm{Co} 2$ & N11 & $94.53(8)$ & $\mathrm{C} 7$ & $\mathrm{C} 8$ & C9 & $119.8(2)$ \\
\hline N7 & $\mathrm{Co} 2$ & N12 & $175.31(8)$ & $\mathrm{C} 8$ & C9 & $\mathrm{C} 11$ & $123.9(2)$ \\
\hline N8 & $\mathrm{Co} 2$ & N4 & $92.89(8)$ & $\mathrm{C} 10$ & C9 & $\mathrm{C} 8$ & $117.3(2)$ \\
\hline N11 & $\mathrm{Co} 2$ & N4 & $176.14(8)$ & $\mathrm{C} 10$ & C9 & $\mathrm{C} 11$ & $118.5(2)$ \\
\hline N11 & $\mathrm{Co} 2$ & N8 & $88.68(8)$ & N2 & $\mathrm{C} 10$ & C9 & $123.3(2)$ \\
\hline N11 & $\mathrm{Co} 2$ & $\mathrm{~N} 12$ & $83.33(8)$ & $\mathrm{C} 12$ & $\mathrm{C} 11$ & C9 & $120.7(2)$ \\
\hline N12 & $\mathrm{Co} 2$ & N4 & $93.08(8)$ & $\mathrm{C} 16$ & $\mathrm{C} 11$ & C9 & $119.8(2)$ \\
\hline N12 & $\mathrm{Co} 2$ & N8 & $92.21(8)$ & $\mathrm{C} 16$ & $\mathrm{C} 11$ & $\mathrm{C} 12$ & $119.1(2)$ \\
\hline $\mathrm{C} 1$ & N1 & Co1 & $127.02(16)$ & $\mathrm{C} 13$ & $\mathrm{C} 12$ & $\mathrm{C} 11$ & $120.6(2)$ \\
\hline $\mathrm{C} 1$ & $\mathrm{~N} 1$ & $\mathrm{C} 5$ & $118.72(19)$ & $\mathrm{C} 12$ & $\mathrm{C} 13$ & $\mathrm{C} 14$ & $120.4(2)$ \\
\hline $\mathrm{C} 5$ & N1 & Co1 & $114.24(15)$ & $\mathrm{C} 13$ & $\mathrm{C} 14$ & $\mathrm{C} 25$ & $119.1(2)$ \\
\hline C6 & N2 & Co1 & $114.55(15)$ & $\mathrm{C} 15$ & $\mathrm{C} 14$ & $\mathrm{C} 13$ & $118.6(2)$ \\
\hline $\mathrm{C} 10$ & N2 & Co1 & $125.98(15)$ & $\mathrm{C} 15$ & $\mathrm{C} 14$ & $\mathrm{C} 25$ & $121.8(2)$ \\
\hline $\mathrm{C} 10$ & N2 & C6 & $118.72(19)$ & $\mathrm{C} 16$ & $\mathrm{C} 15$ & $\mathrm{C} 14$ & $121.0(2)$ \\
\hline $\mathrm{C} 17$ & N3 & $\mathrm{Co} 2$ & $126.31(16)$ & $\mathrm{C} 15$ & $\mathrm{C} 16$ & $\mathrm{C} 11$ & $120.1(2)$ \\
\hline $\mathrm{C} 17$ & $\mathrm{~N} 3$ & $\mathrm{C} 21$ & $118.9(2)$ & N3 & $\mathrm{C} 17$ & $\mathrm{C} 18$ & $121.8(2)$ \\
\hline $\mathrm{C} 21$ & N3 & $\mathrm{Co} 2$ & $114.74(15)$ & $\mathrm{C} 19$ & $\mathrm{C} 18$ & $\mathrm{C} 17$ & $119.5(2)$ \\
\hline $\mathrm{C} 22$ & N4 & $\mathrm{Co} 2$ & $114.51(15)$ & $\mathrm{C} 18$ & C19 & $\mathrm{C} 20$ & $119.0(2)$ \\
\hline $\mathrm{C} 26$ & N4 & $\mathrm{Co} 2$ & $125.58(15)$ & $\mathrm{C} 21$ & $\mathrm{C} 20$ & $\mathrm{C} 19$ & $119.3(2)$ \\
\hline $\mathrm{C} 26$ & N4 & $\mathrm{C} 22$ & 119.27(19) & N3 & $\mathrm{C} 21$ & $\mathrm{C} 20$ & $121.6(2)$ \\
\hline $\mathrm{C} 27$ & N5 & Co1 & $126.80(16)$ & N3 & $\mathrm{C} 21$ & $\mathrm{C} 22$ & $113.68(19)$ \\
\hline $\mathrm{C} 27$ & N5 & C31 & $118.8(2)$ & $\mathrm{C} 20$ & $\mathrm{C} 21$ & $\mathrm{C} 22$ & $124.7(2)$ \\
\hline C31 & N5 & Co1 & $114.38(15)$ & N4 & $\mathrm{C} 22$ & $\mathrm{C} 21$ & $113.86(19)$ \\
\hline C32 & N6 & Co1 & $114.51(15)$ & N4 & $\mathrm{C} 22$ & $\mathrm{C} 23$ & $120.9(2)$ \\
\hline $\mathrm{C} 36$ & N6 & Co1 & $126.23(15)$ & $\mathrm{C} 23$ & $\mathrm{C} 22$ & $\mathrm{C} 21$ & $125.0(2)$ \\
\hline C36 & N6 & C32 & $118.88(19)$ & $\mathrm{C} 24$ & $\mathrm{C} 23$ & $\mathrm{C} 22$ & $119.8(2)$ \\
\hline $\mathrm{C} 43$ & N7 & $\mathrm{Co} 2$ & 126.61(16) & $\mathrm{C} 23$ & $\mathrm{C} 24$ & $\mathrm{C} 25$ & $119.6(2)$ \\
\hline $\mathrm{C} 43$ & N7 & $\mathrm{C} 47$ & $119.1(2)$ & $\mathrm{C} 24$ & $\mathrm{C} 25$ & $\mathrm{C} 14$ & $123.7(2)$ \\
\hline $\mathrm{C} 47$ & N7 & $\mathrm{Co} 2$ & $114.20(15)$ & $\mathrm{C} 26$ & $\mathrm{C} 25$ & $\mathrm{C} 14$ & $118.6(2)$ \\
\hline $\mathrm{C} 48$ & $\mathrm{~N} 8$ & $\mathrm{Co} 2$ & $114.19(15)$ & $\mathrm{C} 26$ & $\mathrm{C} 25$ & $\mathrm{C} 24$ & $117.3(2)$ \\
\hline C52 & N8 & $\mathrm{Co} 2$ & $125.98(15)$ & N4 & $\mathrm{C} 26$ & $\mathrm{C} 25$ & $123.0(2)$ \\
\hline C52 & $\mathrm{N} 8$ & $\mathrm{C} 48$ & $119.06(19)$ & N5 & $\mathrm{C} 27$ & $\mathrm{C} 28$ & $122.0(2)$ \\
\hline C53 & N9 & Co1 & $126.84(16)$ & $\mathrm{C} 27$ & $\mathrm{C} 28$ & $\mathrm{C} 29$ & $119.1(2)$ \\
\hline
\end{tabular}




\begin{tabular}{|c|c|c|c|c|c|c|c|}
\hline Atom & Atom & Atom & Angle $/^{\circ}$ & Atom & Atom & Atom & Angle $/^{\circ}$ \\
\hline $\mathrm{C} 30$ & C29 & C28 & $119.3(2)$ & $\overline{\mathrm{C} 68}$ & C67 & C66 & $120.4(2)$ \\
\hline $\mathrm{C} 29$ & $\mathrm{C} 30$ & C31 & $118.9(2)$ & C67 & C68 & C63 & $120.2(2)$ \\
\hline N5 & C31 & C30 & $121.8(2)$ & N11 & C69 & C70 & $121.4(2)$ \\
\hline N5 & C31 & C32 & $113.68(19)$ & C71 & C70 & C69 & $119.8(2)$ \\
\hline $\mathrm{C} 30$ & $\mathrm{C} 31$ & $\mathrm{C} 32$ & $124.5(2)$ & $\mathrm{C} 70$ & C71 & C72 & $119.2(2)$ \\
\hline N6 & $\mathrm{C} 32$ & C31 & $113.86(19)$ & $\mathrm{C} 73$ & C72 & C71 & $118.6(2)$ \\
\hline N6 & $\mathrm{C} 32$ & C33 & $121.0(2)$ & N11 & C73 & C72 & $121.9(2)$ \\
\hline $\mathrm{C} 33$ & $\mathrm{C} 32$ & C31 & $124.9(2)$ & N11 & C73 & C74 & $113.75(19)$ \\
\hline C34 & $\mathrm{C} 33$ & $\mathrm{C} 32$ & $119.8(2)$ & $\mathrm{C} 72$ & C73 & C74 & $124.3(2)$ \\
\hline C33 & $\mathrm{C} 34$ & $\mathrm{C} 35$ & $119.3(2)$ & $\mathrm{N} 12$ & C74 & C73 & $113.75(19)$ \\
\hline C34 & $\mathrm{C} 35$ & C37 & $124.1(2)$ & N12 & C74 & C75 & $121.0(2)$ \\
\hline $\mathrm{C} 36$ & C35 & C34 & $117.7(2)$ & C75 & C74 & C73 & $125.1(2)$ \\
\hline $\mathrm{C} 36$ & $\mathrm{C} 35$ & $\mathrm{C} 37$ & $117.9(2)$ & C76 & C75 & C74 & $119.1(2)$ \\
\hline N6 & $\mathrm{C} 36$ & $\mathrm{C} 35$ & $123.0(2)$ & C75 & C76 & C77 & $120.2(2)$ \\
\hline C38 & C37 & $\mathrm{C} 35$ & $121.0(2)$ & C76 & C77 & C66 & $123.7(2)$ \\
\hline C38 & C37 & $\mathrm{C} 42$ & $119.1(2)$ & $\mathrm{C} 78$ & C77 & C66 & $118.6(2)$ \\
\hline $\mathrm{C} 42$ & C37 & $\mathrm{C} 35$ & $119.4(2)$ & C78 & C77 & C76 & $117.2(2)$ \\
\hline C39 & $\mathrm{C} 38$ & C37 & $120.5(2)$ & $\mathrm{N} 12$ & $\mathrm{C} 78$ & C77 & $123.0(2)$ \\
\hline C38 & C39 & $\mathrm{C} 40$ & $120.6(2)$ & $\mathrm{F} 1$ & B1 & F2 & $109.3(2)$ \\
\hline C39 & $\mathrm{C} 40$ & C51 & $119.3(2)$ & $\mathrm{F} 1$ & B1 & F4 & $109.3(2)$ \\
\hline $\mathrm{C} 41$ & $\mathrm{C} 40$ & C39 & $118.8(2)$ & F3 & B1 & F1 & $109.6(2)$ \\
\hline $\mathrm{C} 41$ & $\mathrm{C} 40$ & C51 & $121.7(2)$ & F3 & B1 & $\mathrm{F} 2$ & $109.4(2)$ \\
\hline $\mathrm{C} 42$ & $\mathrm{C} 41$ & $\mathrm{C} 40$ & $120.7(2)$ & F3 & B1 & F4 & $109.4(2)$ \\
\hline C41 & $\mathrm{C} 42$ & C37 & $120.2(2)$ & $\mathrm{F} 4$ & B1 & $\mathrm{F} 2$ & $109.8(2)$ \\
\hline N7 & $\mathrm{C} 43$ & $\mathrm{C} 44$ & $121.7(2)$ & F5 & B2 & F7 & $108.4(3)$ \\
\hline $\mathrm{C} 45$ & $\mathrm{C} 44$ & $\mathrm{C} 43$ & $119.1(2)$ & F5 & B2 & F8 & $108.5(2)$ \\
\hline $\mathrm{C} 46$ & $\mathrm{C} 45$ & $\mathrm{C} 44$ & $119.4(2)$ & F6 & B2 & F5 & $111.2(2)$ \\
\hline $\mathrm{C} 47$ & $\mathrm{C} 46$ & $\mathrm{C} 45$ & $118.9(2)$ & F6 & B2 & F7 & $109.3(2)$ \\
\hline N7 & $\mathrm{C} 47$ & $\mathrm{C} 46$ & $121.6(2)$ & F6 & B2 & F8 & $113.5(3)$ \\
\hline N7 & $\mathrm{C} 47$ & $\mathrm{C} 48$ & $113.84(19)$ & F8 & B2 & F7 & $105.7(2)$ \\
\hline $\mathrm{C} 46$ & $\mathrm{C} 47$ & $\mathrm{C} 48$ & $124.5(2)$ & F9A & B3 & F10A & $96.7(5)$ \\
\hline N8 & $\mathrm{C} 48$ & $\mathrm{C} 47$ & $114.16(19)$ & F9A & B3 & F12 & $107.8(5)$ \\
\hline N8 & $\mathrm{C} 48$ & C49 & $121.0(2)$ & F10 & B3 & F9 & $107.3(6)$ \\
\hline C49 & $\mathrm{C} 48$ & $\mathrm{C} 47$ & $124.5(2)$ & F10 & B3 & F11 & $118.6(6)$ \\
\hline $\mathrm{C} 50$ & C49 & $\mathrm{C} 48$ & $119.4(2)$ & F10 & B3 & F12 & $111.4(4)$ \\
\hline C49 & $\mathrm{C} 50$ & C51 & $120.1(2)$ & F11 & B3 & F9 & $104.6(3)$ \\
\hline $\mathrm{C} 50$ & C51 & $\mathrm{C} 40$ & $123.5(2)$ & F11 & B3 & F12 & $107.5(3)$ \\
\hline C52 & C51 & $\mathrm{C} 40$ & $119.2(2)$ & $\mathrm{F} 11 \mathrm{~A}$ & B3 & F9A & $112.2(6)$ \\
\hline C52 & C51 & C50 & $117.0(2)$ & F11A & B3 & F10A & $111.2(5)$ \\
\hline N8 & C52 & C51 & $123.1(2)$ & F11A & B3 & F12 & $117.5(3)$ \\
\hline N9 & C53 & C54 & $122.3(2)$ & F12 & B3 & F9 & $106.7(3)$ \\
\hline C53 & C54 & C55 & $119.3(2)$ & F12 & B3 & F10A & $109.5(3)$ \\
\hline C54 & C55 & C56 & $118.7(2)$ & F13 & B4 & F14 & $104.7(2)$ \\
\hline $\mathrm{C} 57$ & C56 & C55 & $119.4(2)$ & F13 & B4 & F15 & $114.5(3)$ \\
\hline N9 & C57 & C56 & $121.8(2)$ & F14 & B4 & F15 & $107.6(2)$ \\
\hline N9 & C57 & C58 & $113.82(19)$ & F16 & B4 & F13 & $108.0(3)$ \\
\hline C56 & $\mathrm{C} 57$ & C58 & $124.4(2)$ & F16 & B4 & F14 & $113.9(2)$ \\
\hline N10 & C58 & C57 & $114.03(19)$ & F16 & B4 & F15 & $108.2(2)$ \\
\hline N10 & C58 & C59 & $121.2(2)$ & F17 & B5 & F18 & $108.6(2)$ \\
\hline C59 & C58 & C57 & $124.6(2)$ & F17 & B5 & F19 & $109.7(2)$ \\
\hline C60 & C59 & C58 & $119.4(2)$ & F19 & B5 & F18 & $108.8(2)$ \\
\hline C59 & C60 & C61 & $119.7(2)$ & F20 & B5 & F17 & $110.4(2)$ \\
\hline C60 & C61 & C62 & $117.3(2)$ & $\mathrm{F} 20$ & B5 & F18 & $109.4(2)$ \\
\hline C60 & C61 & C63 & $124.0(2)$ & $\mathrm{F} 20$ & B5 & F19 & $109.9(2)$ \\
\hline C62 & C61 & C63 & $118.4(2)$ & F23 & B6 & $\mathrm{F} 22$ & $110.9(3)$ \\
\hline N10 & C62 & C61 & $123.1(2)$ & F23 & B6 & F24 & $108.4(3)$ \\
\hline C64 & C63 & C61 & $120.9(2)$ & F23 & B6 & F21 & $112.4(3)$ \\
\hline C64 & C63 & C68 & $119.1(2)$ & F22 & B6 & $\mathrm{F} 24$ & $108.0(3)$ \\
\hline C68 & C63 & C61 & $119.7(2)$ & $\mathrm{F} 22$ & B6 & F21 & $109.4(3)$ \\
\hline C65 & C64 & C63 & $120.6(2)$ & F21 & B6 & F24 & $107.7(3)$ \\
\hline C64 & C65 & C66 & $120.4(2)$ & $\mathrm{F} 22 \mathrm{~A}$ & B6A & F21A & $103.0(15)$ \\
\hline C65 & C66 & C77 & $118.5(2)$ & $\mathrm{F} 22 \mathrm{~A}$ & B6A & $\mathrm{F} 23 \mathrm{~A}$ & $109.9(15)$ \\
\hline C67 & C66 & C65 & 119.1(2) & $\mathrm{F} 22 \mathrm{~A}$ & B6A & F24A & $118.9(15)$ \\
\hline C67 & C66 & C77 & $122.0(2)$ & F23A & B6A & $\mathrm{F} 21 \mathrm{~A}$ & $104.6(15)$ \\
\hline
\end{tabular}




\begin{tabular}{llll}
\hline Atom & Atom & Atom & \multicolumn{1}{c}{ Angle $/^{\circ}$} \\
\hline F24A & B6A & F21A & $106.3(15)$ \\
F24A & B6A & F23A & $112.6(14)$ \\
N13 & C79 & C80 & $179.2(4)$ \\
N14 & C81 & C82 & $179.0(3)$ \\
N15 & C83 & C84 & $179.2(4)$
\end{tabular}

\begin{tabular}{llll}
\hline Atom & Atom & Atom & \multicolumn{1}{c}{ Angle $/^{\circ}$} \\
\hline N16 & C85 & C86 & $178.6(4)$ \\
N17 & C87 & C88 & $178.0(5)$ \\
N18 & C89 & C90 & $179.4(3)$ \\
N19 & C91 & C92 & $179.3(4)$
\end{tabular}


Table S16: Hydrogen Fractional Atomic Coordinates $\left(\times 10^{4}\right)$ and Equivalent Isotropic Displacement Parameters $\left(\AA^{2} \times 10^{3}\right)$ for PL15013. $U_{e q}$ is defined as $1 / 3$ of the trace of the orthogonalised $U_{i j}$.

\begin{tabular}{|c|c|c|c|c|}
\hline Atom & & & & $U_{e q}$ \\
\hline$\overline{\mathrm{H} 1}$ & -283 & 1808 & 5300 & 27 \\
\hline $\mathrm{H} 2$ & -1515 & 1961 & 6357 & 32 \\
\hline H3 & -1251 & 2611 & 7132 & 34 \\
\hline H4 & 269 & 3105 & 6821 & 31 \\
\hline $\mathrm{H} 7$ & 1684 & 3676 & 6348 & 29 \\
\hline H8 & 3180 & 4242 & 5741 & 29 \\
\hline H10 & 3740 & 2854 & 4307 & 23 \\
\hline H12 & 5282 & 2955 & 4177 & 28 \\
\hline H13 & 6627 & 3602 & 3306 & 27 \\
\hline H15 & 5451 & 5808 & 3772 & 26 \\
\hline H16 & 4106 & 5160 & 4646 & 26 \\
\hline H17 & 8314 & 8021 & -453 & 31 \\
\hline H18 & 9829 & 7608 & -1168 & 36 \\
\hline H19 & 10672 & 6423 & -684 & 32 \\
\hline $\mathrm{H} 20$ & 9951 & 5658 & 511 & 28 \\
\hline $\mathrm{H} 23$ & 9071 & 4941 & 1622 & 29 \\
\hline $\mathrm{H} 24$ & 8015 & 4275 & 2742 & 30 \\
\hline H26 & 6133 & 6282 & 2598 & 23 \\
\hline $\mathrm{H} 27$ & 1433 & 499 & 4566 & 29 \\
\hline $\mathrm{H} 28$ & 470 & 36 & 4100 & 35 \\
\hline H29 & -469 & 1011 & 3494 & 36 \\
\hline H30 & -319 & 2435 & 3288 & 32 \\
\hline H33 & 148 & 3742 & 2978 & 30 \\
\hline H34 & 773 & 5027 & 2693 & 29 \\
\hline H36 & 2119 & 4040 & 4167 & 25 \\
\hline H38 & 1974 & 5424 & 4200 & 28 \\
\hline H39 & 3007 & 6470 & 3855 & 28 \\
\hline H41 & 3450 & 6648 & 1738 & 26 \\
\hline H42 & 2373 & 5634 & 2084 & 26 \\
\hline H43 & 8821 & 7989 & 964 & 29 \\
\hline H44 & 9222 & 8752 & 1593 & 36 \\
\hline H45 & 8004 & 9262 & 2512 & 39 \\
\hline H46 & 6408 & 8985 & 2788 & 34 \\
\hline H49 & 4980 & 8477 & 3091 & 30 \\
\hline H50 & 3624 & 7756 & 3400 & 29 \\
\hline H52 & 4927 & 6868 & 1620 & 24 \\
\hline H53 & 1219 & 965 & 6103 & 27 \\
\hline H54 & 1859 & -290 & 6653 & 30 \\
\hline H55 & 3340 & -870 & 5999 & 32 \\
\hline H56 & 4134 & -157 & 4796 & 30 \\
\hline H59 & 4838 & 654 & 3695 & 28 \\
\hline H60 & 5505 & 1655 & 2620 & 28 \\
\hline H62 & 2999 & 3062 & 3090 & 24 \\
\hline H64 & 3482 & 3658 & 1928 & 27 \\
\hline H65 & 4043 & 4903 & 1120 & 26 \\
\hline H67 & 6682 & 4208 & 1311 & 27 \\
\hline H68 & 6113 & 2964 & 2132 & 27 \\
\hline H69 & 7096 & 9221 & 564 & 28 \\
\hline $\mathrm{H} 70$ & 6523 & 10280 & -234 & 32 \\
\hline H71 & 5752 & 9956 & -925 & 30 \\
\hline H72 & 5531 & 8555 & -775 & 28 \\
\hline H75 & 5272 & 7193 & -468 & 27 \\
\hline H76 & 4927 & 5799 & 39 & 27 \\
\hline H78 & 6589 & 5586 & 1259 & 23 \\
\hline H1A & 9837 & 4961 & 4704 & 78 \\
\hline H1B & 10126 & 5481 & 5058 & 78 \\
\hline $\mathrm{H} 80 \mathrm{~A}$ & 1438 & 467 & 1496 & 65 \\
\hline H80B & 1342 & 552 & 2279 & 65 \\
\hline $\mathrm{H} 80 \mathrm{C}$ & 630 & 1100 & 1871 & 65 \\
\hline H82A & 2583 & 4129 & 939 & 69 \\
\hline
\end{tabular}




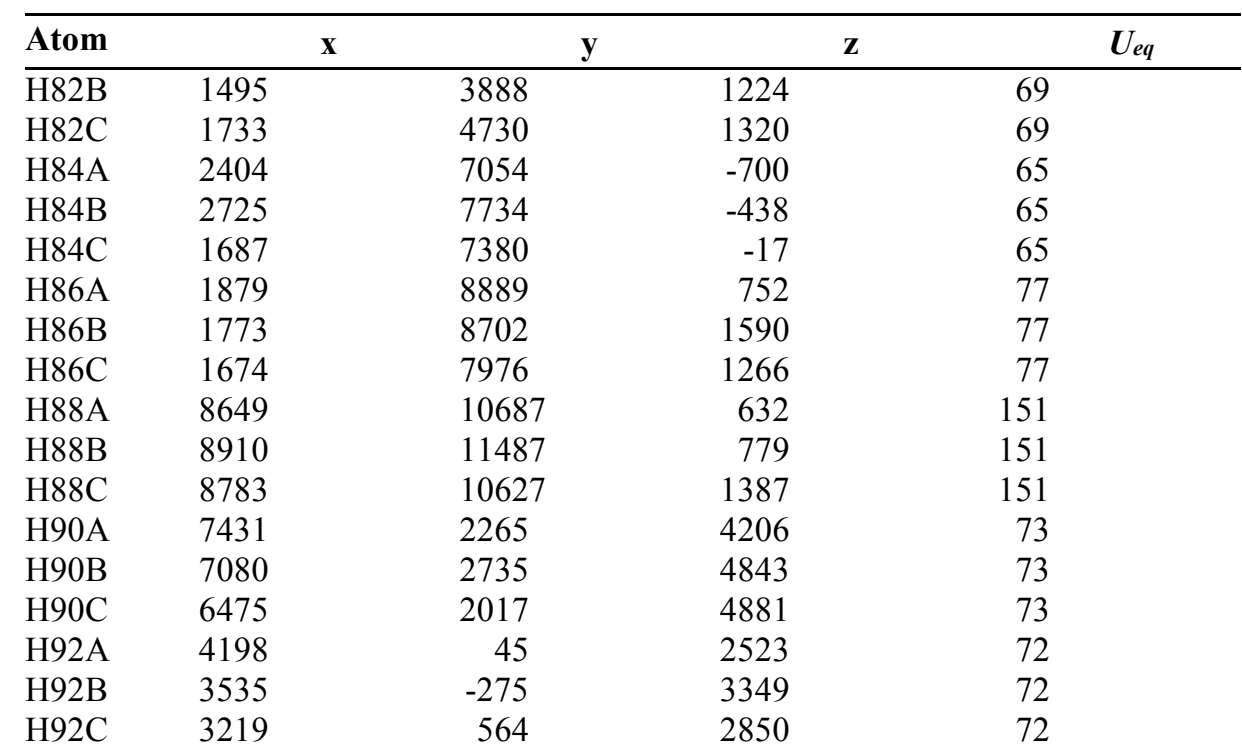

Table S17: Hydrogen Bond information for PL15013.

\begin{tabular}{|c|c|c|c|c|c|c|}
\hline D & H & $\mathbf{A}$ & $\mathrm{d}(\mathrm{D}-\mathrm{H}) / \AA$ & $\mathbf{d}(\mathbf{H}-\mathbf{A}) / \AA ̊$ & $\mathbf{d}(\mathbf{D}-\mathbf{A}) / \AA$ & D-H-A/deg \\
\hline $\mathrm{O} 1$ & H1B & $\mathrm{F}^{11 \mathrm{~A}^{1}}$ & 1.03 & 1.90 & $2.838(8)$ & 149.6 \\
\hline
\end{tabular}


2d·6BF 4 (CCDC 1429784) Crystal Data and Experimental

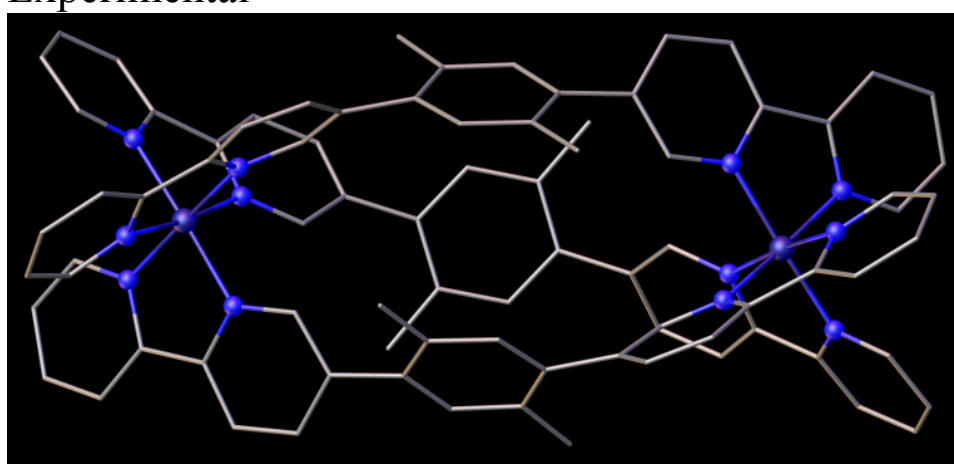

Experimental. Single yellow block-shaped crystals of (pl15025) were recrystallised from a mixture of acetonitrile and diisopropyl ether by vapour diffusion. A suitable crystal $(0.16 \times 0.10 \times 0.06)$ was selected and mounted on a MITIGEN holder in Paratone oil on a Rigaku Oxford Diffraction SuperNova diffractometer. The crystal was kept at $T=$ 120.0 K during data collection. Using Olex2, ${ }^{[3]}$ the structure was solved with the ShelXT structure solution program, ${ }^{[4]}$ using the Direct Methods solution method. The model was refined with version of ShelXL using Least Squares minimisation. ${ }^{[5]}$

Crystal Data. $\mathrm{C}_{96} \mathrm{H}_{84} \mathrm{~B}_{6} \mathrm{Co}_{2} \mathrm{~F}_{24} \mathrm{~N}_{18}, M_{r}=2128.53$, trigonal, R-3c (No. 167), $\mathrm{a}=17.3577(3) \AA, \mathrm{b}=17.3577(3) \AA, \mathrm{c}=$ $104.263(2) \AA, \alpha=90^{\circ}, \beta=90^{\circ}, \gamma=120^{\circ}, V=27204.8(11) \AA^{3}$, $T=120.0 \mathrm{~K}, Z=12, Z^{\prime}=0.33333, \mu\left(\mathrm{CuK}_{\alpha}\right)=3.829,81531$ reflections measured, 3562 unique $\left(R_{\text {int }}=0.1111\right)$ which were used in all calculations. The final $w R_{2}$ was 0.2237 (all data) and $R_{l}$ was $0.0769(\mathrm{I}>2(\mathrm{I}))$.

$\begin{array}{ll}\text { Formula } & \mathrm{C}_{96} \mathrm{H}_{84} \mathrm{~B}_{6} \mathrm{Co}_{2} \mathrm{~F}_{24} \mathrm{~N}_{18} \\ D_{\text {calc. }} / \mathrm{g} \mathrm{cm}^{-3} & 1.559 \\ \mu / \mathrm{mm}^{-1} & 3.829 \\ \text { Formula Weight } & 2128.53 \\ \text { Colour } & \text { yellow } \\ \text { Shape } & \text { block } \\ \text { Max Size/mm } & 0.16 \\ \text { Mid Size/mm } & 0.10 \\ \text { Min Size } / \mathrm{mm} & 0.06 \\ \text { T/K } & 120.0 \\ \text { Crystal System } & \end{array}$

trigonal

Space Group R-3c

$a / \AA$

$17.3577(3)$

$b / \AA$

$17.3577(3)$

$c / \AA$

104.263(2)

$\alpha l^{\circ}$

90

$\beta 1^{\circ}$

90

$\gamma \rho^{\circ}$

120

$\mathrm{V} / \AA^{3}$

27204.8(11)

Z

12

$Z^{\prime}$

0.33333

$\theta_{\min } I^{\circ}$

$\theta_{\max }{ }^{\circ}$

Measured Refl.

81531

Independent Refl.

3562

3030

Reflections Used

$R_{\text {int }}$

0.1111

Parameters

387

Restraints

0

Largest Peak

1.855

Deepest Hole

$-0.639$

GooF

1.047

$w R_{2}$ (all data)

0.2237

0.2137

$w R_{2}$ 


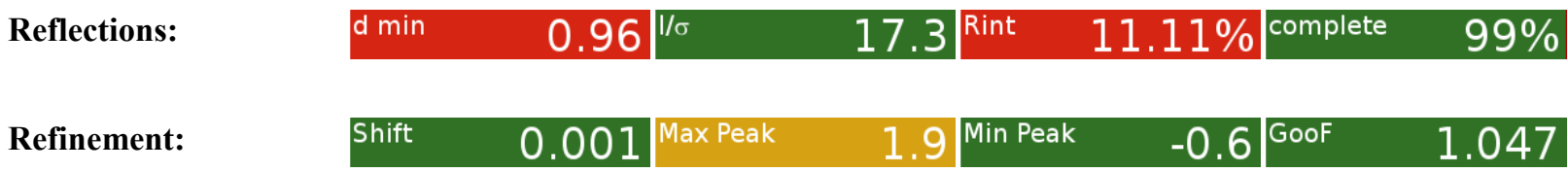

A yellow block-shaped crystal with dimensions $0.16 \times 0.10 \times 0.06$ was mounted on a MITIGEN holder in Paratone oil. Data were collected using a Rigaku Oxford Diffraction SuperNova diffractometer equipped with an Oxford Cryosystems Cryostream 700+ low-temperature apparatus operating at $T=120.0 \mathrm{~K}$.

Data were measured using $\omega$ scans scans of $1.0^{\circ}$ per frame for $10.0 \mathrm{~s}$ using $\mathrm{CuK}_{\square}$ radiation (sealed X-ray tube, $50 \mathrm{kV}, 0.8 \mathrm{~mA}$ ). The total number of runs and images was based on the strategy calculation from the program CrysAlisPro (Agilent, V1.171.37.35, 2014). The actually achieved resolution was $\theta=53.410$.

Cell parameters were retrieved using the CrysAlisPro (Agilent, V1.171.37.35, 2014) software and refined using CrysAlisPro (Agilent, V1.171.37.35, 2014) on 16813 reflections, 21 of the observed reflections. Data reduction was performed using the CrysAlisPro (Agilent, V1.171.37.35, 2014) software which corrects for Lorentz polarisation. The final completeness is 99.20 out to 53.410 in $\theta$. The absorption coefficient $(\mu)$ of this material is 3.829 and the minimum and maximum transmissions are 0.2772 and 0.4717 .

The structure was solved in the space group R-3c (\# 167) by Direct Methods using the ShelXT structure solution program and refined by Least Squares using version of ShelXL. ${ }^{[4-5]}$ All non-hydrogen atoms were refined anisotropically. Hydrogen atom positions were calculated geometrically and refined using the riding model. The SQUEEZE routine of PLATON was used to account for electron density corresponding to six molecules of acetonitrile per asymmetric unit. This was accounted for in the chemical formula, triggering checkCIF alerts which should be ignored. The resolution of the data set was cut at $0.96 \AA$ due to rapidly rising merging statistics at higher resolution.

The value of $Z^{\prime}$ is 0.33333 .

\section{Reflection Statistics}

\begin{tabular}{|c|c|c|c|}
\hline \multirow{2}{*}{\multicolumn{2}{|c|}{ Total reflections (after filtering) 86303}} & \multicolumn{2}{|l|}{ Unique reflections } \\
\hline & & & 3562 \\
\hline & 0.992 & & \\
\hline Completeness & & Mean $\mathrm{I} / \sigma$ & 17.32 \\
\hline hklsub $>\max </$ sub $>$ collected & $(18,18,107)$ & hklsub $>\min </$ sub $>$ collected & $(-18,-15,-108)$ \\
\hline $\mathrm{hkl} \mathrm{l}_{\max }$ used & $(0,18,108)$ & $\mathrm{hkl} l_{\min }$ used & $(-15,0,0)$ \\
\hline Lim $d_{\max }$ collected & 100.0 & Lim $\mathrm{d}_{\min }$ collected & 0.77 \\
\hline$d_{\max }$ used & 13.02 & $d_{\min }$ used & 0.96 \\
\hline Friedel pairs & 6736 & Friedel pairs merged & 1 \\
\hline Inconsistent equivalents & 0 & $\mathrm{R}_{\text {int }}$ & 0.1111 \\
\hline $\mathrm{R}_{\text {sigma }}$ & 0.0428 & Intensity transformed & 0 \\
\hline Omitted reflections & 0 & Omitted by user (OMIT hkl) & 0 \\
\hline Multiplicity & $\begin{array}{l}(9168,5277,4083,2757,2464, \\
1636,995,484,360,297,184, \\
126,35,5,4)\end{array}$ & Maximum multiplicity & 49 \\
\hline Removed systematic absences & 4772 & Filtered off (Shel/OMIT) & 0 \\
\hline
\end{tabular}

Table S18: Fractional Atomic Coordinates $\left(\times 10^{4}\right)$ and Equivalent Isotropic Displacement Parameters $\left(\AA^{2} \times 10^{3}\right)$ for 
pl15025. $U_{e q}$ is defined as $1 / 3$ of the trace of the orthogonalised $U_{i j}$. 


\begin{tabular}{|c|c|c|c|c|}
\hline Atom & $\mathbf{x}$ & $\mathbf{y}$ & $\mathbf{z}$ & $U_{e q}$ \\
\hline Co1 & 3333 & 6667 & $5130.4(2)$ & $33.1(5)$ \\
\hline $\mathrm{Co} 2$ & 3333 & 6667 & $6243.7(2)$ & $43.6(5)$ \\
\hline F1 & $2829(3)$ & $2518(2)$ & $5513.7(3)$ & $59.7(10)$ \\
\hline F7 & $9(3)$ & $2899(3)$ & $5085.5(4)$ & $\begin{array}{l}68.3(11) \\
76.0(13)\end{array}$ \\
\hline F6 & $883(3)$ & $\begin{array}{l}4256(3) \\
3017(3)\end{array}$ & $5177.3(4)$ & \\
\hline F5 & $738(3)$ & & $\begin{array}{l}5273.1(4) \\
5230.8(4)\end{array}$ & $85.8(14)$ \\
\hline $\mathrm{N} 2$ & $\begin{array}{l}3000(3) \\
3959(3)\end{array}$ & $5610(3)$ & & $34.2(11)$ \\
\hline N1 & & $6229(3)$ & $5027.2(4)$ & $35.9(12)$ \\
\hline $\mathrm{F} 2$ & $4085(3)$ & 3711(3) & $5590.5(5)$ & $93.8(15)$ \\
\hline N3 & $2322(4)$ & $6476(4)$ & $6346.8(4)$ & $47.8(14)$ \\
\hline $\mathrm{F} 4$ & $3999(4)$ & $3270(4)$ & $5387.0(5)$ & $106.2(19)$ \\
\hline N4 & $2427(3)$ & $5688(3)$ & $6144.2(4)$ & $42.9(13)$ \\
\hline F3 & $4051(4)$ & $2425(4)$ & $5549.7(6)$ & $109.0(18)$ \\
\hline $\mathrm{C} 11$ & $2207(4)$ & $4599(4)$ & $5554.6(5)$ & $39.3(14)$ \\
\hline $\mathrm{C} 5$ & $3763(4)$ & $5392(4)$ & $5057.9(5)$ & $39.5(14)$ \\
\hline C6 & $3270(4)$ & $5060(4)$ & $5179.6(5)$ & $37.2(14)$ \\
\hline C9 & $\begin{array}{l}2483(4) \\
1521(4)\end{array}$ & $4680(4)$ & $5416.6(5)$ & $37.3(14)$ \\
\hline F8 & & $3589(5)$ & $5091.0(5)$ & $110.9(19)$ \\
\hline $\mathrm{C} 27$ & $1816(4)$ & $4777(4)$ & $5956.7(5)$ & $45.6(15)$ \\
\hline $\mathrm{C} 16$ & $1479(4)$ & $4660(4)$ & $5597.9(5)$ & $39.6(14)$ \\
\hline $\mathrm{C} 10$ & $2595(4)$ & $5404(4)$ & $5345.1(5)$ & $36.9(14)$ \\
\hline $\mathrm{C} 23$ & $1545(5)$ & $5726(4)$ & $6315.2(5)$ & $47.3(17)$ \\
\hline $\mathrm{C} 1$ & $4455(4)$ & $6617(4)$ & $4922.5(5)$ & $42.9(15)$ \\
\hline $\mathrm{C} 24$ & $1601(4)$ & $5303(4)$ & $6196.2(5)$ & $47.0(16)$ \\
\hline C12 & $2769(4)$ & $4521(4)$ & $5642.6(5)$ & $41.2(14)$ \\
\hline $\mathrm{C} 28$ & $2533(4)$ & $5411(4)$ & $6027.9(5)$ & $43.7(15)$ \\
\hline $\mathrm{C} 8$ & $\begin{array}{l}2740(4) \\
1953(4)\end{array}$ & $4112(4)$ & $5362.0(5)$ & $44.0(15)$ \\
\hline $\mathrm{C} 14$ & & $4637(4)$ & $5819.1(5)$ & $43.6(15)$ \\
\hline C13 & $2668(4)$ & $4548(4)$ & $5774.2(5)$ & $44.3(15)$ \\
\hline C15 & $1364(4)$ & $4669(4)$ & $5730.7(5)$ & $41.5(14)$ \\
\hline C18 & $810(4)$ & $4693(5)$ & $5509.1(6)$ & $50.5(16)$ \\
\hline $\mathrm{C} 2$ & $4743(4)$ & $6178(5)$ & $4843.3(5)$ & $50.4(17)$ \\
\hline $\mathrm{C} 7$ & $3133(4)$ & $4304(4)$ & $5242.4(5)$ & $44.0(15)$ \\
\hline $\mathrm{C} 25$ & $871(5)$ & $4630(4)$ & $6133.9(6)$ & $51.7(17)$ \\
\hline
\end{tabular}




\begin{tabular}{|c|c|c|c|c|}
\hline Atom & $\mathbf{x}$ & $\mathbf{y}$ & $\mathbf{Z}$ & $U_{e q}$ \\
\hline $\mathrm{C} 4$ & $3999(4)$ & $\begin{array}{l}4909(4) \\
5419(5)\end{array}$ & $4980.4(6)$ & $47.9(16)$ \\
\hline $\mathrm{C} 22$ & $792(5)$ & & $\begin{array}{l}6388.0(6) \\
5862.2(6)\end{array}$ & $55.2(18)$ \\
\hline $\mathrm{C} 17$ & $\begin{array}{r}3323(5) \\
977(5)\end{array}$ & $4479(5)$ & & $51.5(16)$ \\
\hline $\mathrm{C} 26$ & & $4367(4)$ & $6013.3(6)$ & $50.5(17)$ \\
\hline $\mathrm{C} 3$ & $4490(4)$ & $5301(5)$ & $4869.9(6)$ & $51.8(17)$ \\
\hline $\mathrm{C} 20$ & $1587(5)$ & $6692(5)$ & $6523.7(6)$ & $58.7(18)$ \\
\hline $\mathrm{C} 21$ & $816(5)$ & $5898(5)$ & $6496.4(6)$ & $57.7(18)$ \\
\hline C19 & $2325(5)$ & $6956(5)$ & $6449.1(5)$ & $52.6(17)$ \\
\hline B1 & $3753(6)$ & $2959(6)$ & $5511.7(8)$ & $60(2)$ \\
\hline B2 & $700(7)$ & $3432(7)$ & $5167.0(9)$ & $73(3)$ \\
\hline
\end{tabular}

Table S19: Anisotropic Displacement Parameters $\left(\times 10^{4}\right)$ pl15025. The anisotropic displacement factor exponent takes the form: $-2 \pi^{2}\left[h^{2} a^{* 2} \times U_{11}+\ldots+2 h k a * \times b^{*} \times U_{12}\right]$

\begin{tabular}{lllcccc}
\hline Atom & $\boldsymbol{U}_{11}$ & $\boldsymbol{U}_{22}$ & $\boldsymbol{U}_{33}$ & $\boldsymbol{U}_{23}$ & $\boldsymbol{U}_{13}$ & $\boldsymbol{U}_{12}$ \\
\hline Co1 & $40.6(7)$ & $40.6(7)$ & $17.9(8)$ & 0 & 0 & $20.3(3)$ \\
C02 & $56.4(8)$ & $56.4(8)$ & $18.0(9)$ & 0 & 0 & $28.2(4)$ \\
F1 & $69(3)$ & $60(2)$ & $51(2)$ & $13.7(18)$ & $19.8(19)$ & $33(2)$ \\
F7 & $67(3)$ & $86(3)$ & $51(2)$ & $-15(2)$ & $-8(2)$ & $39(2)$ \\
F6 & $76(3)$ & $69(3)$ & $66(3)$ & $3(2)$ & $-18(2)$ & $23(2)$
\end{tabular}




\begin{tabular}{|c|c|c|c|c|c|c|}
\hline Atom & $U_{11}$ & $U_{22}$ & $U_{33}$ & $\boldsymbol{U}_{23}$ & $U_{13}$ & $U_{12}$ \\
\hline F5 & $82(3)$ & $81(3)$ & $69(3)$ & $11(2)$ & $-13(2)$ & $21(3)$ \\
\hline N2 & $38(3)$ & $43(3)$ & $21(2)$ & $0(2)$ & $0(2)$ & $20(2)$ \\
\hline N1 & $\begin{array}{l}38(3) \\
92(3)\end{array}$ & $49(3)$ & $20(2)$ & $-2(2)$ & $0(2)$ & $21(2)$ \\
\hline $\mathrm{F} 2$ & & $83(3)$ & $106(4)$ & $-9(3)$ & $-11(3)$ & $43(3)$ \\
\hline N3 & $64(4)$ & $62(4)$ & $21(3)$ & $1(2)$ & $5(2)$ & $34(3)$ \\
\hline F4 & $109(4)$ & $153(5)$ & $74(3)$ & $47(3)$ & $52(3)$ & $78(4)$ \\
\hline N4 & $54(3)$ & $52(3)$ & $19(2)$ & $1(2)$ & $-1(2)$ & $24(3)$ \\
\hline F3 & $112(4)$ & $107(4)$ & $138(5)$ & $27(4)$ & $4(4)$ & $78(4)$ \\
\hline $\mathrm{C} 11$ & $52(4)$ & $37(3)$ & $25(3)$ & $1(2)$ & $0(3)$ & $19(3)$ \\
\hline C5 & $49(4)$ & $44(4)$ & $28(3)$ & $-3(3)$ & $0(3)$ & $26(3)$ \\
\hline C6 & $45(3)$ & $42(3)$ & $24(3)$ & $-4(3)$ & $-1(2)$ & $21(3)$ \\
\hline C9 & $42(3)$ & $40(3)$ & $26(3)$ & $0(3)$ & $2(2)$ & $17(3)$ \\
\hline F8 & $90(4)$ & $164(6)$ & $83(3)$ & $-25(3)$ & $-5(3)$ & $68(4)$ \\
\hline $\mathrm{C} 27$ & $60(4)$ & $47(4)$ & $29(3)$ & $7(3)$ & $3(3)$ & $25(3)$ \\
\hline C16 & $43(4)$ & $41(3)$ & $27(3)$ & $0(2)$ & $-2(3)$ & $15(3)$ \\
\hline $\mathrm{C} 10$ & $39(3)$ & $44(3)$ & $\begin{array}{l}23(3) \\
22(3)\end{array}$ & $1(3)$ & $0(2)$ & $18(3)$ \\
\hline $\mathrm{C} 23$ & $61(4)$ & $60(4)$ & & $3(3)$ & $3(3)$ & $31(4)$ \\
\hline $\mathrm{C} 1$ & $48(4)$ & $54(4)$ & $28(3)$ & $6(3)$ & $7(3)$ & $26(3)$ \\
\hline $\mathrm{C} 24$ & $59(4)$ & $54(4)$ & $27(3)$ & $9(3)$ & $2(3)$ & $28(4)$ \\
\hline $\mathrm{C} 12$ & $53(4)$ & $42(3)$ & $29(3)$ & $\begin{array}{l}6(3) \\
0(3)\end{array}$ & $5(3)$ & $23(3)$ \\
\hline C28 & $59(4)$ & $48(4)$ & $\begin{array}{l}21(3) \\
36(3)\end{array}$ & & $0(3)$ & $25(3)$ \\
\hline $\mathrm{C} 8$ & $53(4)$ & $39(3)$ & & $4(3)$ & $-1(3)$ & $20(3)$ \\
\hline C14 & $57(4)$ & $40(3)$ & $27(3)$ & $1(3)$ & $3(3)$ & $20(3)$ \\
\hline C13 & $61(4)$ & $40(3)$ & $25(3)$ & $6(3)$ & $1(3)$ & $20(3)$ \\
\hline C15 & $51(4)$ & $44(4)$ & $27(3)$ & $\begin{array}{l}-1(3) \\
-5(3)\end{array}$ & $2(3)$ & $21(3)$ \\
\hline C18 & $47(4)$ & $67(4)$ & $35(3)$ & & $-4(3)$ & $27(3)$ \\
\hline $\mathrm{C} 2$ & $55(4)$ & $76(5)$ & $26(3)$ & $3(3)$ & $5(3)$ & $38(4)$ \\
\hline C7 & $61(4)$ & $42(4)$ & $32(3)$ & $2(3)$ & $3(3)$ & $28(3)$ \\
\hline $\mathrm{C} 25$ & $58(4)$ & $56(4)$ & $31(3)$ & $7(3)$ & $8(3)$ & $21(4)$ \\
\hline $\mathrm{C} 4$ & $65(4)$ & $55(4)$ & $35(3)$ & $\begin{array}{r}-4(3) \\
5(3)\end{array}$ & $2(3)$ & $39(3)$ \\
\hline \multirow{2}{*}{$\begin{array}{l}\mathrm{C} 22 \\
\mathrm{C} 17\end{array}$} & $61(5)$ & $76(5)$ & $29(3)$ & & $2(3)$ & $35(4)$ \\
\hline & $69(4)$ & $65(4)$ & $31(3)$ & $4(3)$ & $0(3)$ & $42(4)$ \\
\hline
\end{tabular}




\begin{tabular}{lllcccc}
\hline Atom & $\boldsymbol{U}_{11}$ & $\boldsymbol{U}_{22}$ & $\boldsymbol{U}_{3 \mathbf{3}}$ & $\boldsymbol{U}_{23}$ & $\boldsymbol{U}_{\mathbf{1 3}}$ & $\boldsymbol{U}_{\mathbf{1 2}}$ \\
\hline $\mathrm{C} 26$ & $62(4)$ & $48(4)$ & $29(3)$ & $0(3)$ & $1(3)$ & $18(3)$ \\
$\mathrm{C} 3$ & $59(4)$ & $73(5)$ & $35(4)$ & $-1(3)$ & $8(3)$ & $42(4)$ \\
$\mathrm{C} 20$ & $83(5)$ & $73(5)$ & $28(3)$ & $-5(3)$ & $-1(4)$ & $45(5)$ \\
$\mathrm{C} 21$ & $72(5)$ & $80(5)$ & $29(3)$ & $4(3)$ & & $45(4)$ \\
$\mathrm{C} 19$ & $62(4)$ & $68(5)$ & $23(3)$ & $-5(3)$ & $3(3)$ & $29(4)$ \\
B1 & $73(6)$ & $71(6)$ & $45(5)$ & $10(4)$ & $20(4)$ & $43(5)$ \\
B2 & $101(7)$ & $79(7)$ & $61(6)$ & $-31(5)$ & $-51(5)$ & $61(6)$
\end{tabular}

Table S20: Bond Lengths in $\AA$ for pl15025.

\begin{tabular}{|c|c|c|}
\hline Atom & Atom & Length/Å \\
\hline Co1 & $\mathrm{N} 2^{1}$ & $1.932(5)$ \\
\hline Col & $\mathrm{N} 2^{2}$ & $1.932(5)$ \\
\hline Col & N2 & $1.932(5)$ \\
\hline Col & $\mathrm{N} 1^{2}$ & $1.934(5)$ \\
\hline Col & $\mathrm{N} 1^{1}$ & $1.934(5)$ \\
\hline Col & N1 & $1.934(4)$ \\
\hline $\mathrm{Co} 2$ & $\mathrm{~N} 3^{1}$ & $1.940(5)$ \\
\hline $\mathrm{Co} 2$ & $\mathrm{~N} 3^{2}$ & $1.940(5)$ \\
\hline $\mathrm{Co} 2$ & N3 & $\begin{array}{l}1.940(5) \\
1.940(5)\end{array}$ \\
\hline $\mathrm{Co} 2$ & N4 & \\
\hline $\mathrm{Co} 2$ & $\mathrm{~N} 4^{1}$ & $1.940(5)$ \\
\hline $\mathrm{Co} 2$ & $\mathrm{~N} 4^{2}$ & $1.940(5)$ \\
\hline $\mathrm{F} 1$ & B1 & $1.389(10)$ \\
\hline
\end{tabular}




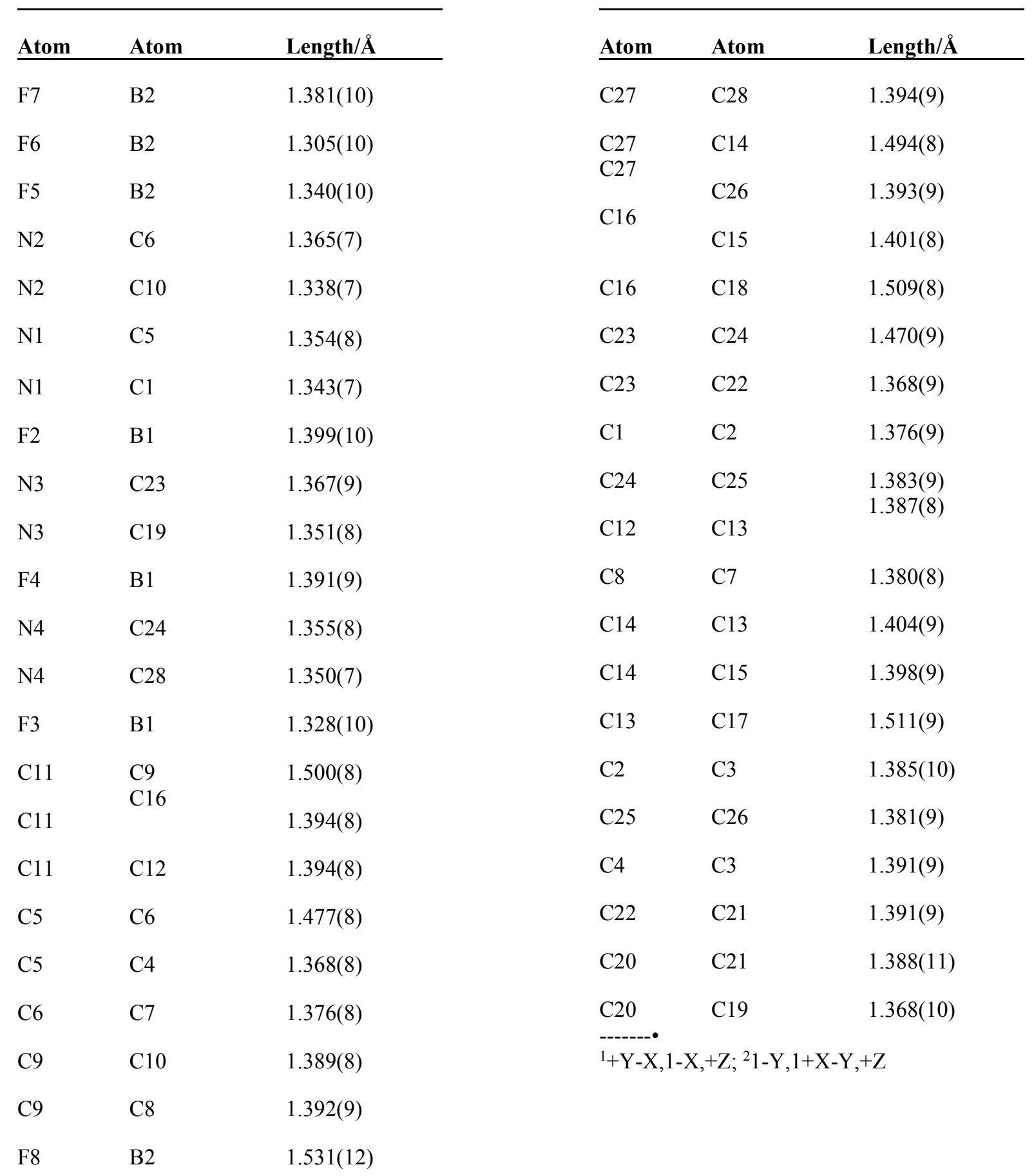

Table S21: Bond Angles in for pl15025. 


\begin{tabular}{|c|c|c|c|c|c|c|c|}
\hline Atom & Atom & Atom & Angle $/^{\circ}$ & Atom & Atom & Atom & Angle $/^{\circ}$ \\
\hline $\mathrm{N} 2^{1}$ & Co1 & $\mathrm{N} 2^{2}$ & $93.46(18)$ & $\mathrm{C} 1$ & N1 & Col & $126.9(4)$ \\
\hline $\mathrm{N} 2^{1}$ & Col & N2 & $93.45(19)$ & $\mathrm{C} 1$ & N1 & $\mathrm{C} 5$ & $118.9(5)$ \\
\hline $\mathrm{N} 2^{2}$ & Co1 & N2 & $93.45(18)$ & $\mathrm{C} 23$ & N3 & $\mathrm{Co} 2$ & $114.0(4)$ \\
\hline $\mathrm{N} 2^{2}$ & Col & $\mathrm{N} 1^{2}$ & $83.61(19)$ & C19 & N3 & $\mathrm{Co} 2$ & $127.4(5)$ \\
\hline N2 & Col & N1 & 83.61(19) & C19 & N3 & $\mathrm{C} 23$ & $118.4(6)$ \\
\hline $\mathrm{N} 2^{1}$ & Col & $\mathrm{N} 1^{2}$ & $91.14(19)$ & $\mathrm{C} 24$ & N4 & $\mathrm{Co} 2$ & $114.4(4)$ \\
\hline $\mathrm{N} 2$ & Col & $\mathrm{N} 1^{2}$ & $174.69(19)$ & $\mathrm{C} 28$ & N4 & $\mathrm{Co} 2$ & $126.6(4)$ \\
\hline $\mathrm{N} 2^{1}$ & Co1 & N1 & $174.7(2)$ & $\mathrm{C} 28$ & N4 & $\mathrm{C} 24$ & $118.8(5)$ \\
\hline $\mathrm{N} 2^{2}$ & $\mathrm{Co} 1$ & N1 & $91.13(19)$ & $\mathrm{C} 16$ & $\mathrm{C} 11$ & C9 & $124.2(5)$ \\
\hline $\mathrm{N} 2^{2}$ & $\mathrm{Co} 1$ & $\mathrm{~N} 1^{1}$ & $174.69(19)$ & $\mathrm{C} 16$ & $\mathrm{C} 11$ & $\mathrm{C} 12$ & $119.9(5)$ \\
\hline $\mathrm{N} 2$ & Co1 & $\mathrm{N} 1^{1}$ & $91.14(19)$ & $\mathrm{C} 12$ & $\mathrm{C} 11$ & C9 & $115.8(5)$ \\
\hline $\mathrm{N} 2^{1}$ & Col & $\mathrm{N} 1^{1}$ & 83.61(19) & N1 & $\mathrm{C} 5$ & C6 & $114.1(5)$ \\
\hline N1 & Co1 & $\mathrm{N} 1^{1}$ & $92.02(19)$ & N1 & $\mathrm{C} 5$ & $\mathrm{C} 4$ & $121.8(5)$ \\
\hline N1 & Col & $\mathrm{N} 1^{2}$ & $92.02(19)$ & $\mathrm{C} 4$ & $\mathrm{C} 5$ & C6 & $124.1(6)$ \\
\hline $\mathrm{N} 1^{2}$ & $\mathrm{Co} 1$ & $\mathrm{~N} 1^{1}$ & 92.01(19) & N2 & C6 & $\mathrm{C} 5$ & $113.4(5)$ \\
\hline N3 & $\mathrm{Co} 2$ & $\mathrm{~N} 3^{2}$ & $92.3(2)$ & $\mathrm{N} 2$ & C6 & $\mathrm{C} 7$ & $121.6(5)$ \\
\hline N3 & $\mathrm{Co} 2$ & $\mathrm{~N} 3^{1}$ & (12.0. & $\mathrm{C} 7$ & C6 & $\mathrm{C} 5$ & $124.9(5)$ \\
\hline $\mathrm{N} 3^{1}$ & $\mathrm{Co} 2$ & $\mathrm{~N} 3^{2}$ & $92.3(2)$ & $\mathrm{C} 10$ & C9 & $\mathrm{C} 11$ & $119.6(5)$ \\
\hline N4 & $\mathrm{Co} 2$ & $\mathrm{~N} 3^{1}$ & $174.4(2)$ & $\mathrm{C} 10$ & C9 & $\mathrm{C} 8$ & $118.2(5)$ \\
\hline $\mathrm{N} 4^{2}$ & $\mathrm{Co} 2$ & N3 & $174.4(2)$ & C8 & C9 & $\mathrm{C} 11$ & $121.7(5)$ \\
\hline $\mathrm{N} 4^{2}$ & $\mathrm{Co} 2$ & $N 3^{2}$ & $83.0(2)$ & $\mathrm{C} 28$ & $\mathrm{C} 27$ & C14 & $119.2(6)$ \\
\hline N4 & $\mathrm{Co} 2$ & N3 & $83.0(2)$ & $\mathrm{C} 26$ & $\mathrm{C} 27$ & $\mathrm{C} 28$ & 118.1(6) \\
\hline $\mathrm{N} 4^{1}$ & $\mathrm{Co} 2$ & N3 & $90.9(2)$ & $\mathrm{C} 26$ & $\mathrm{C} 27$ & $\mathrm{C} 14$ & $122.3(6)$ \\
\hline $\mathrm{N} 4^{2}$ & $\mathrm{Co} 2$ & $\mathrm{~N} 3^{1}$ & & C11 & $\mathrm{C} 16$ & $\mathrm{C} 15$ & $117.4(5)$ \\
\hline N4 & $\mathrm{Co} 2$ & $\mathrm{~N} 3^{2}$ & $90.9(2)$ & & $\mathrm{C} 16$ & C18 & $123.3(5)$ \\
\hline $\mathrm{N} 4^{1}$ & $\mathrm{Co} 2$ & $\mathrm{~N} 3^{2}$ & $174.4(2)$ & & & & \\
\hline $\mathrm{N} 4^{1}$ & $\mathrm{Co} 2$ & $\mathrm{~N} 3^{1}$ & $83.0(2)$ & & & & \\
\hline N4 & $\mathrm{Co} 2$ & $\mathrm{~N} 4^{1}$ & $94.06(19)$ & & & & \\
\hline N4 & $\mathrm{Co} 2$ & $\mathrm{~N} 4^{2}$ & $94.07(19)$ & & & & \\
\hline $\mathrm{N} 4^{1}$ & $\mathrm{Co} 2$ & $\mathrm{~N} 4^{2}$ & $94.06(19)$ & & & & \\
\hline C6 & N2 & Co1 & 114.1(4) & & & & \\
\hline $\mathrm{C} 10$ & $\mathrm{~N} 2$ & Col & $127.1(4)$ & & & & \\
\hline $\mathrm{C} 10$ & N2 & C6 & $118.7(5)$ & & & & \\
\hline $\mathrm{C} 5$ & N1 & Col & $113.6(4)$ & & & & \\
\hline
\end{tabular}




\begin{tabular}{|c|c|c|c|c|c|c|c|}
\hline Atom & Atom & Atom & Angle $/^{\circ}$ & Atom & Atom & Atom & Angle $/^{\circ}$ \\
\hline $\mathrm{C} 15$ & $\mathrm{C} 16$ & $\mathrm{C} 18$ & $119.3(5)$ & $\mathrm{C} 23$ & $\mathrm{C} 22$ & $\mathrm{C} 21$ & $118.8(7)$ \\
\hline N2 & $\mathrm{C} 10$ & C9 & $122.5(5)$ & $\mathrm{C} 25$ & $\mathrm{C} 26$ & $\mathrm{C} 27$ & $119.6(6)$ \\
\hline N3 & $\mathrm{C} 23$ & $\mathrm{C} 24$ & $113.5(5)$ & $\mathrm{C} 2$ & $\mathrm{C} 3$ & $\mathrm{C} 4$ & $119.0(6)$ \\
\hline N3 & $\mathrm{C} 23$ & $\mathrm{C} 22$ & $122.2(6)$ & C19 & $\mathrm{C} 20$ & $\mathrm{C} 21$ & $119.5(6)$ \\
\hline $\mathrm{C} 22$ & $\mathrm{C} 23$ & $\mathrm{C} 24$ & $124.3(6)$ & $\mathrm{C} 20$ & $\mathrm{C} 21$ & $\mathrm{C} 22$ & 119.1(7) \\
\hline N1 & $\mathrm{C} 1$ & $\mathrm{C} 2$ & $122.0(6)$ & N3 & C19 & $\mathrm{C} 20$ & $121.9(7)$ \\
\hline N4 & $\mathrm{C} 24$ & $\mathrm{C} 23$ & $114.2(6)$ & $\mathrm{F} 1$ & B1 & $\mathrm{F} 2$ & $109.3(6)$ \\
\hline N4 & $\mathrm{C} 24$ & $\mathrm{C} 25$ & $121.7(6)$ & $\mathrm{F} 1$ & B1 & $\mathrm{F} 4$ & $105.9(7)$ \\
\hline $\mathrm{C} 25$ & $\mathrm{C} 24$ & $\mathrm{C} 23$ & $123.8(6)$ & F4 & B1 & $\mathrm{F} 2$ & $106.5(7)$ \\
\hline $\mathrm{C} 13$ & $\mathrm{C} 12$ & $\mathrm{C} 11$ & $122.8(6)$ & F3 & B1 & $\mathrm{F} 1$ & $110.8(7)$ \\
\hline N4 & $\mathrm{C} 28$ & $\mathrm{C} 27$ & $122.2(6)$ & F3 & B1 & $\mathrm{F} 2$ & $110.7(7)$ \\
\hline $\mathrm{C} 7$ & $\mathrm{C} 8$ & C9 & $119.5(5)$ & F3 & B1 & $\mathrm{F} 4$ & $113.4(7)$ \\
\hline C13 & $\mathrm{C} 14$ & $\mathrm{C} 27$ & $124.0(6)$ & F7 & B2 & F8 & $102.7(6)$ \\
\hline $\mathrm{C} 15$ & $\mathrm{C} 14$ & $\mathrm{C} 27$ & $116.5(6)$ & F6 & B2 & F7 & $116.0(7)$ \\
\hline $\mathrm{C} 15$ & C14 & $\begin{array}{l}\mathrm{C} 13 \\
\mathrm{C} 14\end{array}$ & $119.3(5)$ & F6 & B2 & F5 & $118.1(7)$ \\
\hline $\mathrm{C} 12$ & $\mathrm{C} 13$ & & $117.8(6)$ & F6 & B2 & F8 & $98.8(8)$ \\
\hline C12 & $\mathrm{C} 13$ & $\mathrm{C} 17$ & $119.0(6)$ & F5 & B2 & F7 & $114.0(8)$ \\
\hline $\mathrm{C} 14$ & $\mathrm{C} 13$ & $\mathrm{C} 17$ & $123.2(5)$ & F5 & B2 & F8 & $103.4(7)$ \\
\hline $\mathrm{C} 14$ & $\mathrm{C} 15$ & $\mathrm{C} 16$ & $122.7(6)$ & \multicolumn{4}{|c|}{${ }^{1+}+\mathrm{Y}-\mathrm{X}, 1-\mathrm{X},+\mathrm{Z} ;{ }^{2} 1-\mathrm{Y}, 1+\mathrm{X}-\mathrm{Y},+\mathrm{Z}$} \\
\hline $\mathrm{C} 1$ & $\mathrm{C} 2$ & $\mathrm{C} 3$ & $119.0(6)$ & & & & \\
\hline C6 & $\begin{array}{l}\mathrm{C} 7 \\
\mathrm{C} 25\end{array}$ & $\mathrm{C} 8$ & $119.3(6)$ & & & & \\
\hline $\begin{array}{l}\mathrm{C} 26 \\
\mathrm{C} 5\end{array}$ & $\mathrm{C} 4$ & $\mathrm{C} 24$ & $119.4(6)$ & & & & \\
\hline
\end{tabular}


Table S22: Hydrogen Fractional Atomic Coordinates $\left(\times 10^{4}\right)$ and Equivalent Isotropic Displacement Parameters $\left(\AA^{2} \times 10^{3}\right)$ for pl15025. $U_{e q}$ is defined as $1 / 3$ of the trace of the orthogonalised $U_{i j}$.

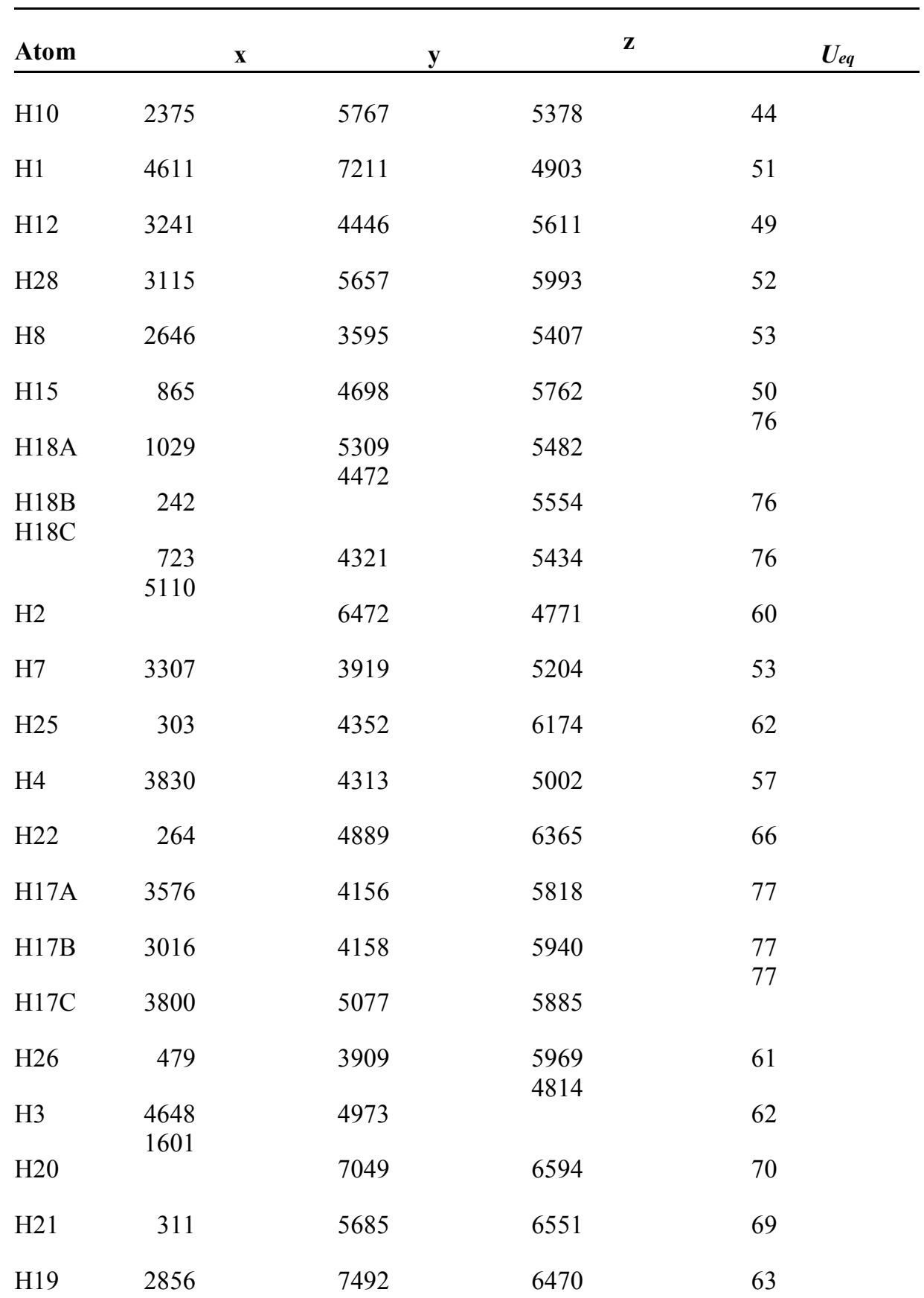




\section{References}

[1] J. Chen, M. Kuss-Petermann, O. S. Wenger, Chem. Eur. J. 2014, 20, 40984104.

[2] P. R. Symmers, M. J. Burke, D. P. August, P. I. T. Thomson, G. S. Nichol, M. R. Warren, C. J. Campbell, P. J. Lusby, Chem. Sci. 2015, 6, 756-760.

[3] O. V. Dolomanov, L. J. Bourhis, R. J. Gildea, J. A. K. Howard, H. Puschmann, J. Appl. Crystallogr. 2009, 42, 339-341.

[4] G. Sheldrick, Acta Crystallographica Section A 2015, 71, 3-8.

[5] G. Sheldrick, Acta Crystallographica Section A 2008, 64, 112-122. 